\title{
Superconducting High Energy Resolution Gamma-ray Spectrometers
}

\author{
D. T. Chow \\ Ph.D Dissertation
}

February 22, 2002

U.S. Department of Energy

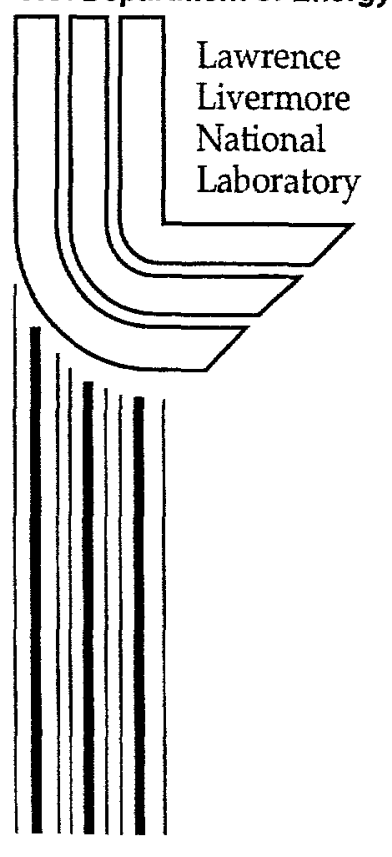


Superconducting High Energy Resolution Gamma-ray Spectrometers

By

DANIEL TUN LAI CHOW

B.S. (Pacific Union College) 1992

M.S. (San Francisco State University) 1995

M.S. (University of California, Davis) 1996

DISSERTATION

Submitted in partial satisfaction of the requirements for the degree of

DOCTOR OF PHILOSOPHY

in

Engineering, Applied Science

in the

OFFICE OF GRADUATE STUDIES

of the

UNIVERSITY OF CALIFORNIA

DAVIS

Approved:

Committee in Charge

2002 
Copyright by

DANIEL TUN LAI CHOW

2002 


\section{Disclaimer}

This document was prepared as an account of work sponsored by an agency of the United States Government. Neither the United States Government nor the University of California nor any of their employees, makes any warranty, express or implied, or assumes any legal liability or responsibility for the accuracy, completeness, or usefulness of any information, apparatus, product, or process disclosed, or represents that its use would not infringe privately owned rights. Reference herein to any specific commercial product, process, or service by trade name, trademark, manufacturer, or otherwise, does not necessarily constitute or imply its endorsement, recommendation, or favoring by the United States Government or the University of California. The views and opinions of authors expressed herein do not necessarily state or reflect those of the United States Government or the University of California, and shall not be used for advertising or product endorsement purposes.

This work was performed under the auspices of the U.S. Department of Energy by the University of California, Lawrence Livermore National Laboratory under Contract W7405-ENG-48. 


\section{ACKNOWLEDGEMENTS}

The pursuit of higher education requires a strong sense of commitment, an infinite amount of patience, and a thorough understanding of the subject. It is important for the student to possess these qualities. It is even more important that the student's colleagues, peers, and loved ones possess these qualities (of course, some peers and loved ones may wish to try to understand the student instead of the subject). So I hereby acknowledge all those who have committed to me, been patient with me, and tried to understand me (or the research project, whichever is easier).

At The Lab: Simon Labov, our fearless leader, for providing direction and guidance (and funding). Matthias Frank, for pointing out that sometimes it is easier to just do it than to complain about having to do it. Marcel van den Berg, for giving honest opinions. Alex Loshak, for keeping the project alive. Larry Hiller, for endless trivia on endless subjects. Stephan Friedrich, for being himself. Joel Ullom, for keeping me alert for "chair quakes" and random projectiles. Mark Cunningham, for being the slacker that he is. Owen Drury, for being anything but dreary. Christie Shannon, whose company I will never admit to enjoy. 
At D.A.S.: Archie Johnson, Estelle Miller, Donna Clifford, and Dee Kindelt, without whom the department will not function. Prof. Ann Orel, for truly being an advocate of the students. Prof. Rick Freeman and Prof. Dennis Matthews, for keeping me motivated.

At Home: Mom (1936-2000), for teaching me the value of critical thinking. Dad, for infinite support even though he still has no idea what I do. Grandpa (1911-1998), for challenging me with scientific questions since I was ten. Grandma, whose genes guarantee my longevity.

And most important of all, Christina (Wifey!), who entered my heart and soul in August, 1995 and has been there ever since. All this I dedicate to you. 


\section{TABLE OF Contents}

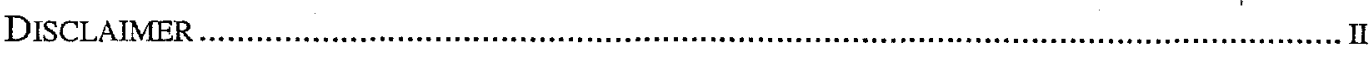

ACKNOWLEDGEMENTS ............................................................................................

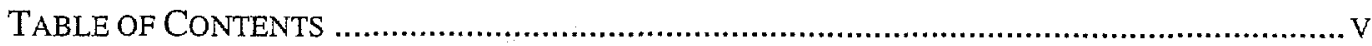

LIST OF FIGURES …..........................................................................................

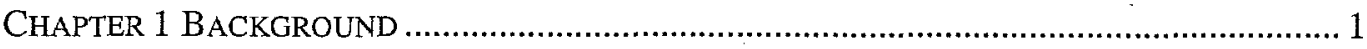

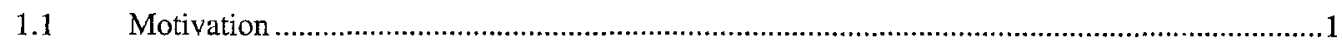

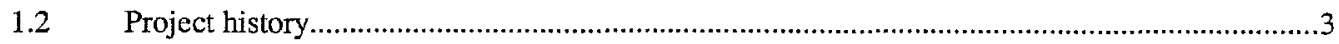

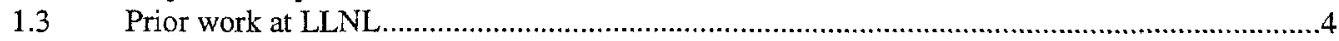

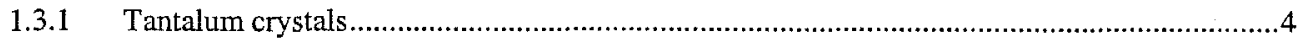

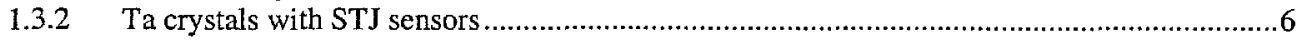

1.3.3 Ta crystals with TES

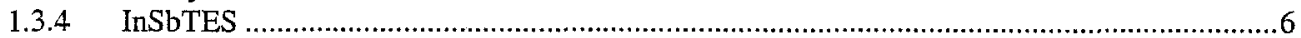

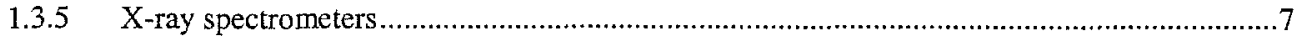

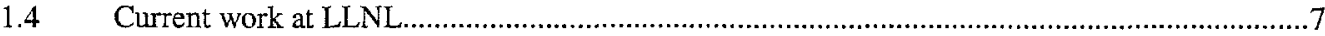

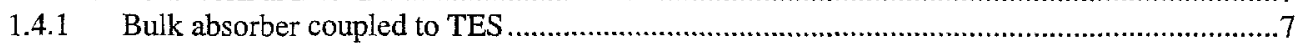

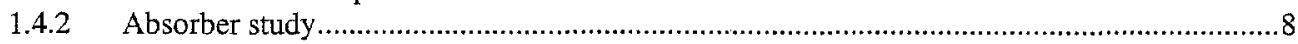

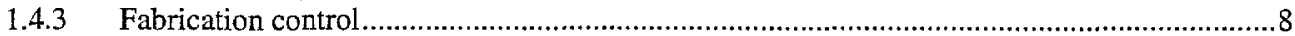

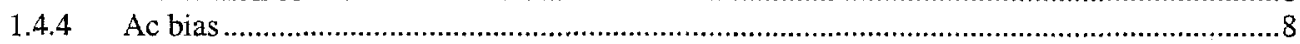

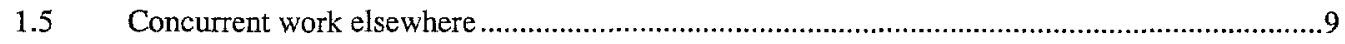

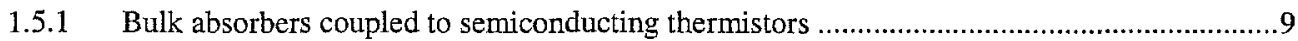

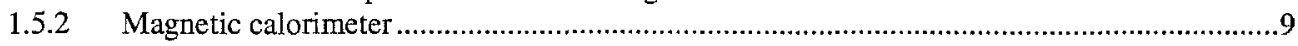

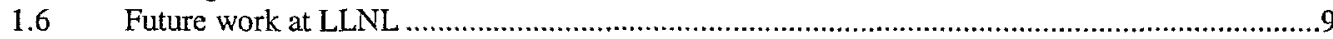

CHAPTER 2 INTRODUCTION TO ENERGY DISPERSIVE DETECTORS..................................... 12

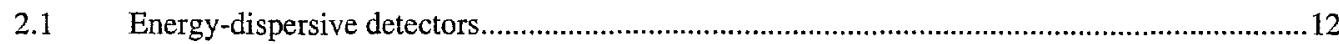

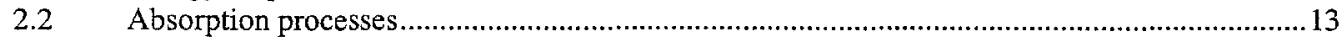

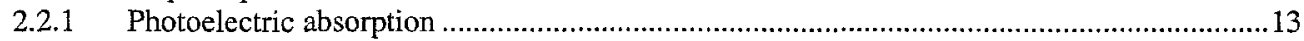

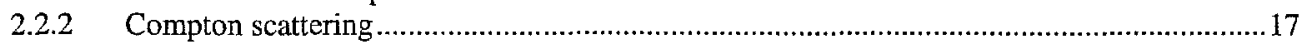

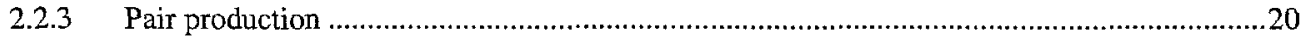

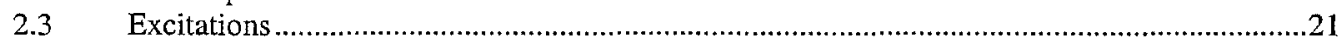

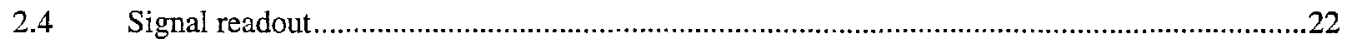

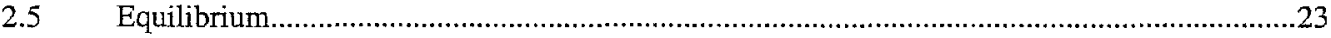

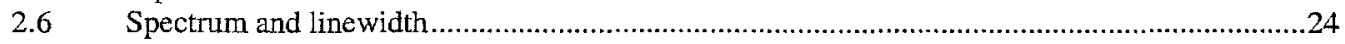

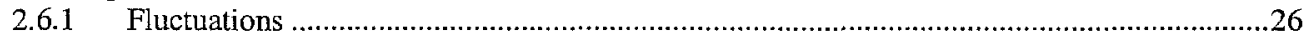

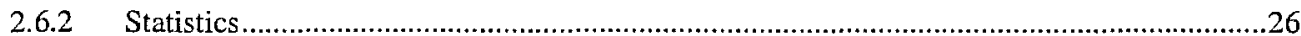

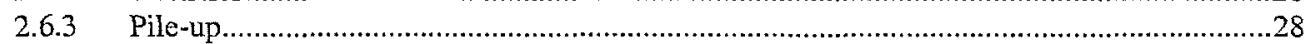

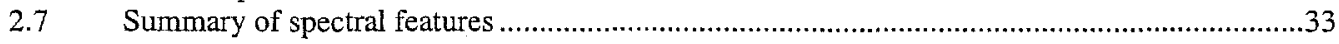

CHAPTER 3 MICROCALORIMETERS AND THE TES ........................................................... 36 


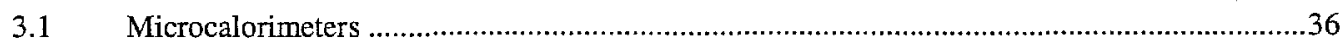

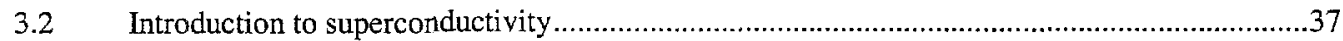

3.2.1 Phenomenon: perfect conductivity and perfect diamagnetism ........................................37

3.2.2 Origin of superconductivity: Coopers pairs and BCS energy gap ....................................40

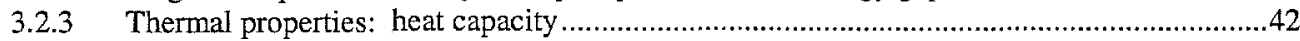

3.2.4 Quantum state: Ginzburg-Landau order parameter ...................................................43

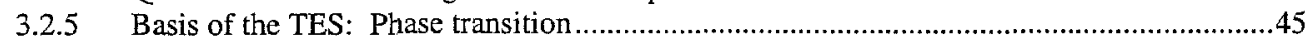

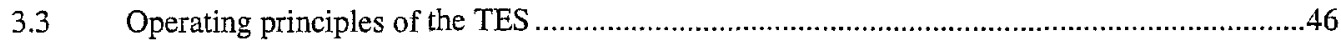

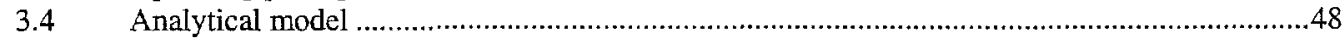

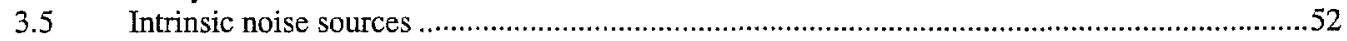

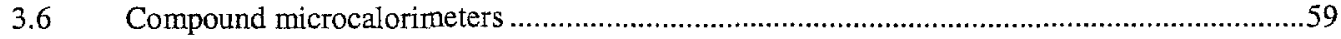

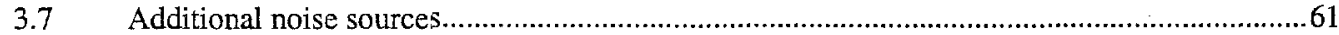

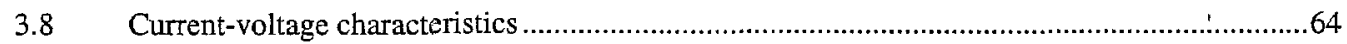

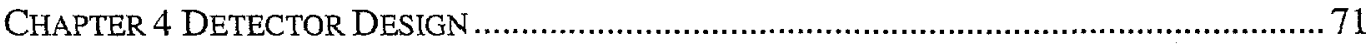

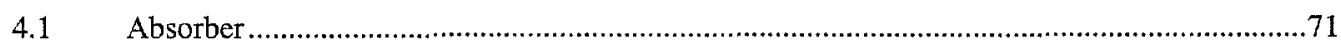

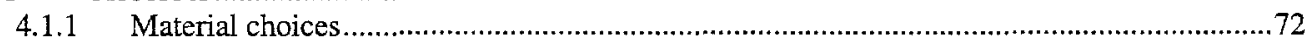

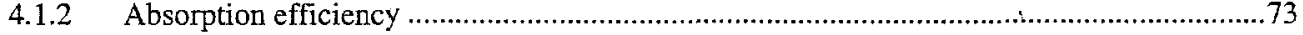

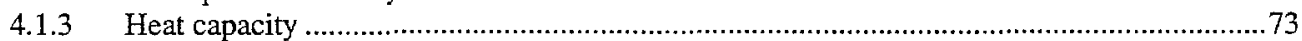

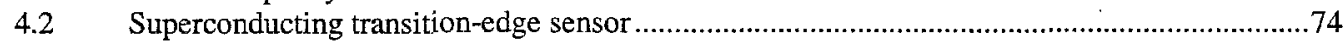

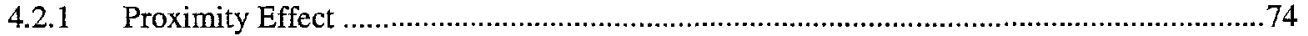

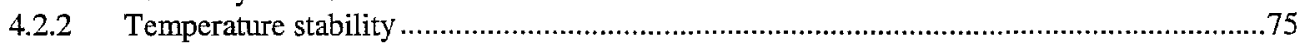

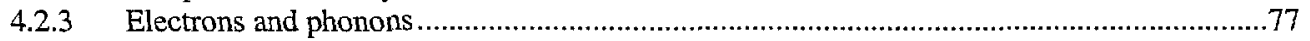

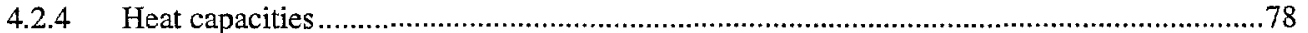

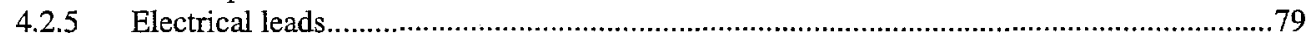

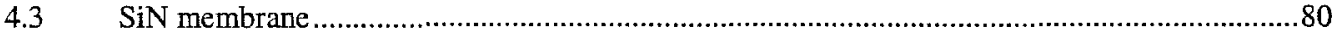

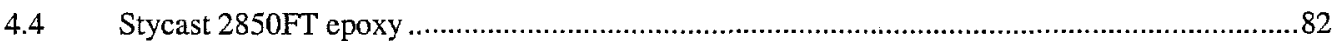

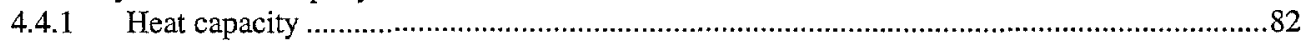

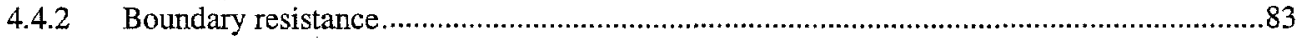

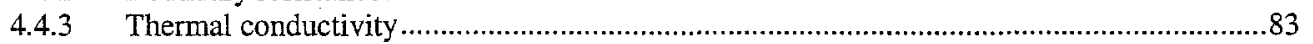

CHAPTER 5 NUMERICAL MODEL ..................................................................... 87

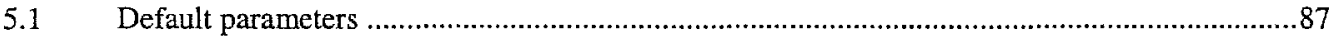

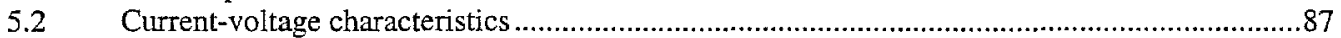

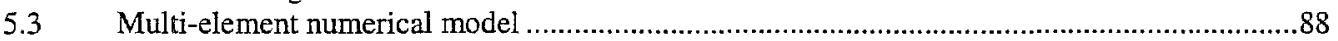

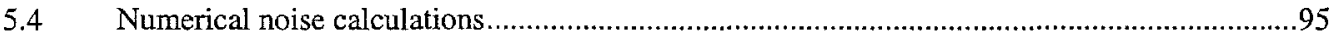

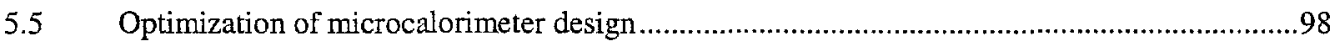

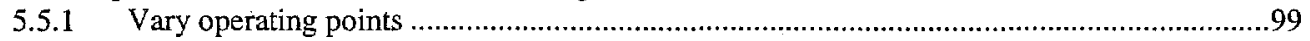

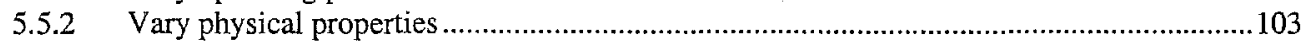

CHAPTER 6 FABRICATION AND TESTING ........................................................ 111

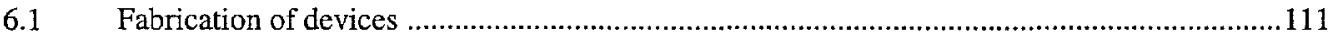

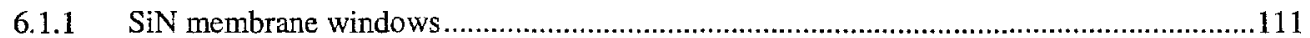

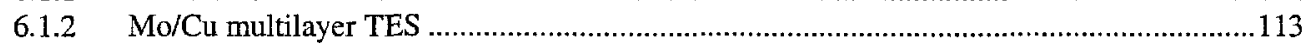

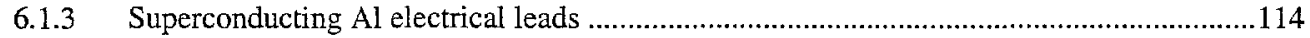

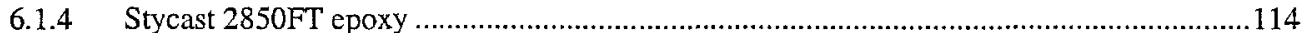

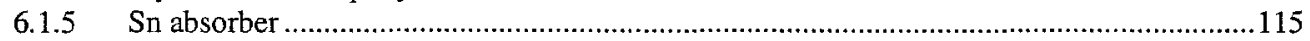

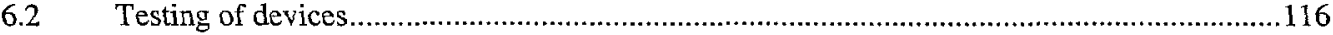

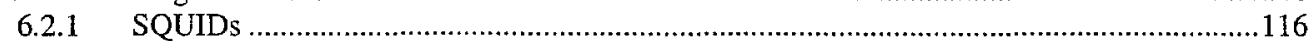

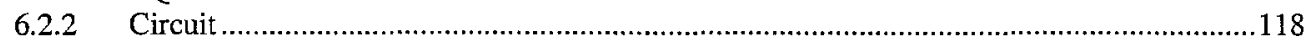

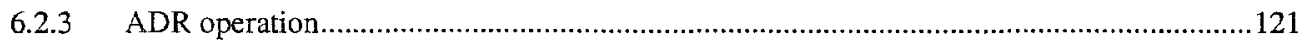

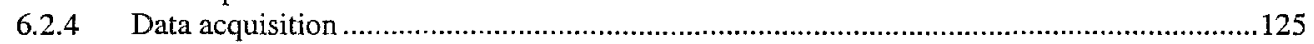

ChaPTER 7 DATA ANALYSIS ...................................................................... 129

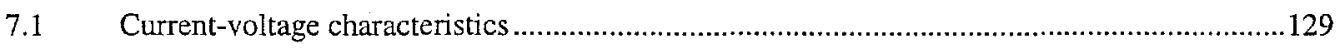




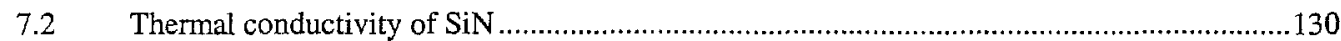

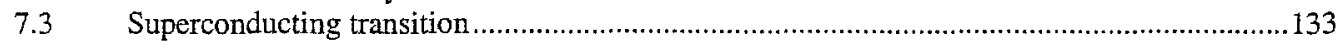

7.4 Current dependence of the superconducting transition....................................................134

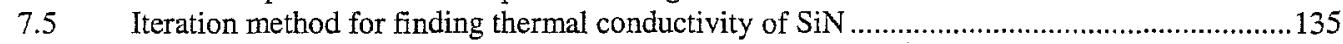

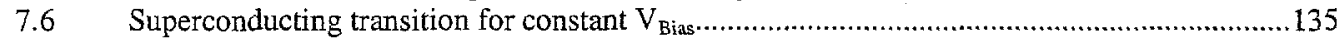

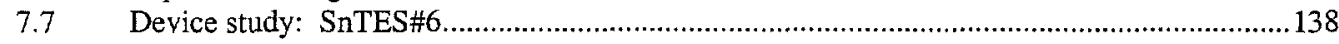

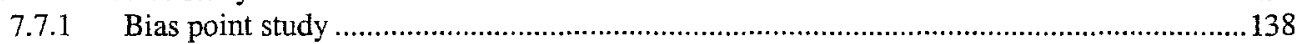

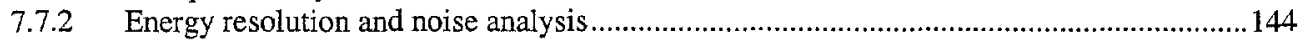

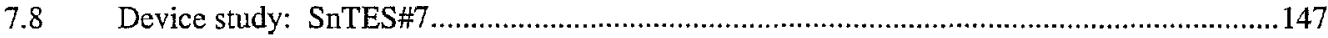

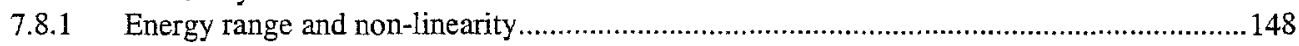

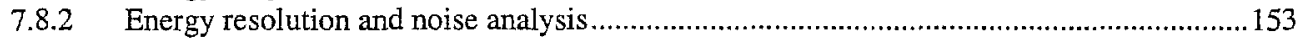

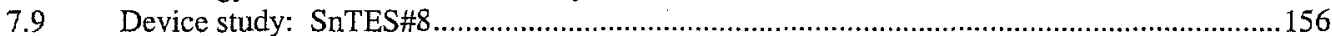

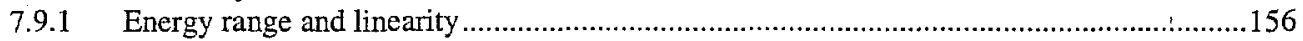

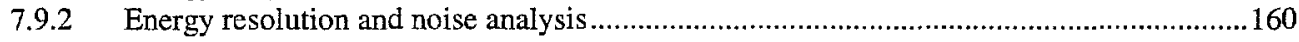

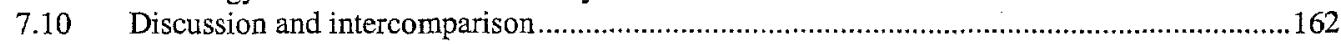

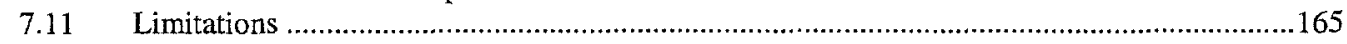

Chapter 8 ACtive Negative FeEdBaCK BIAS ................................................. 167

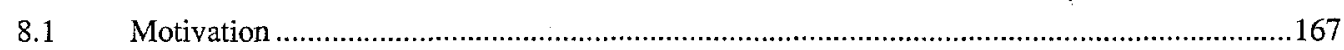

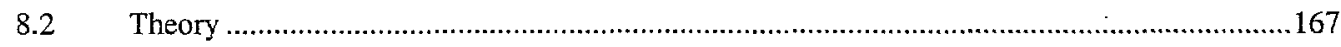

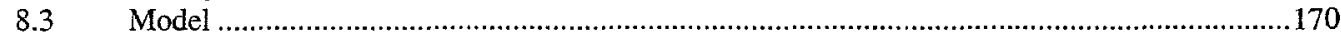

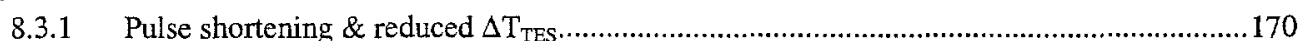

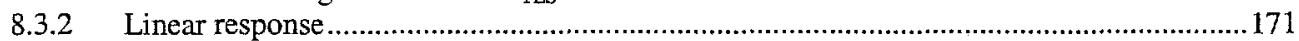

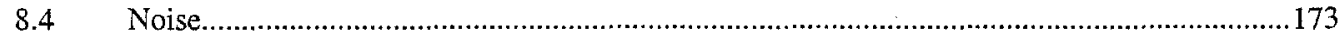

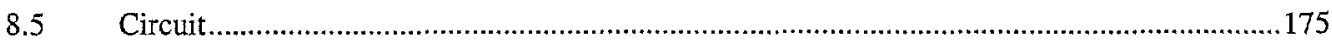

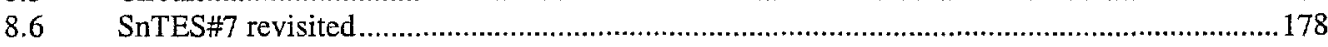

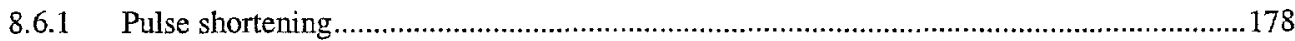

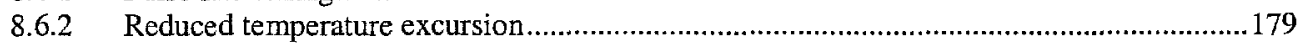

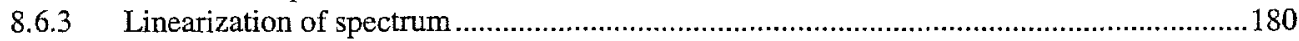

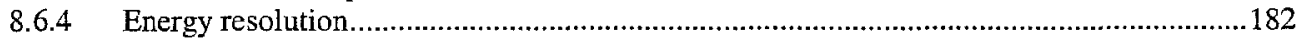

CHAPTER 9 SUMMARY AND CONCLUSIONS ................................................................. 185

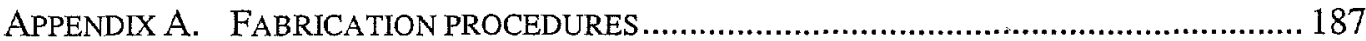




\section{LIST OF FIGURES}

Fig. 1.1 Simulated spectra of $96 \%$ and $98 \%{ }^{235} \mathrm{U}$ enrichment measured with different energy resolution.... 2

Fig. 1.2 Simulated spectrum of ${ }^{235} \mathrm{U}$ against ${ }^{226} \mathrm{Ra}$ background with different energy resolutions............... 3

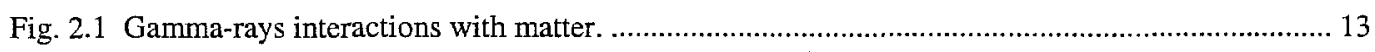

Fig. 2.2 Example of processes following photoelectric absorption. .................................................... 15

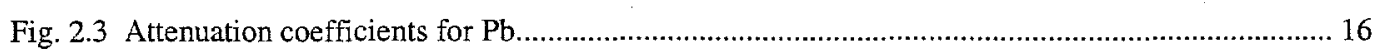

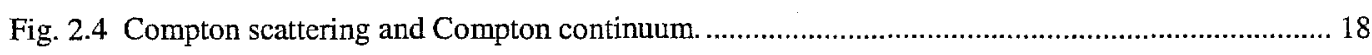

Fig. 2.5 Angular cross section of Compton scattering.............................................................. 19

Fig. 2.6 Feynman diagram illustrating pair production. ...................................................................... 21

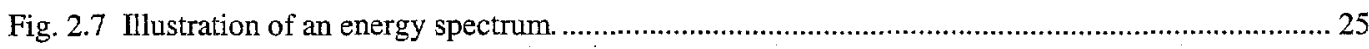

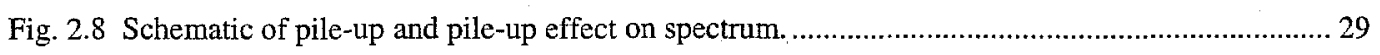

Fig. 2.9 Poisson distribution for pile-up events. ............................................................................ 30

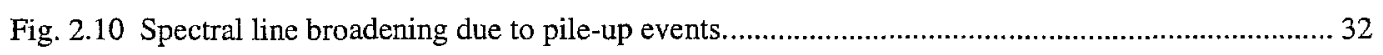

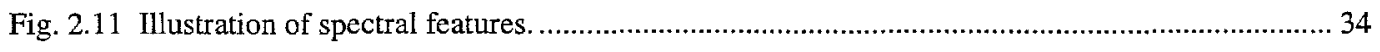

Fig. 3.1 Transition of Hg to superconducting state observed by Kammerlingh Onnes. ........................... 38

Fig. 3.2 Schematic diagram of a TES with operating bias coupled to cold bath. ................................... 49

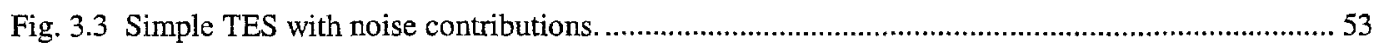

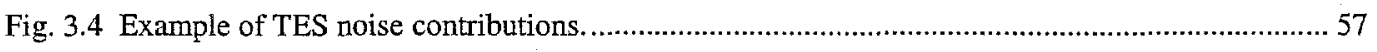

Fig. 3.5 Example of TES noise equivalent power. …................................................................... 59

Fig. 3.6 Schematic diagram of a compound microcalorimeter consisting of a TES and an absorber......... 62

Fig. 3.7 Linear transition from superconducting state to normal state. .................................................6 66

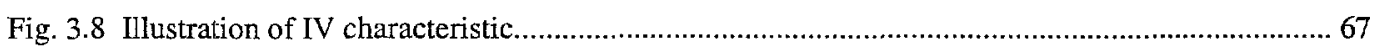

Fig. 3.9 Calculated IV characteristics based on sample TES parameters. ............................................. 68

Fig. 4.1 Illustration of microcalorimeter design in 3D and cross sectional views................................ 72

Fig. 4.2 Calculated absorption efficiency for $250 \mu \mathrm{m}$ thick $\mathrm{Sn}$...................................................... 74

Fig. 4.3 Multilayer transition temperature vs. Cu thickness for fixed Mo thickness of $2 \mathrm{~nm}$................... 76

Fig. 5.1 Modeled IV curves based on default parameters............................................................. 88

Fig. 5.2 Thermal diagram of the 4-component microcalorimeter......................................................... 88

Fig. 5.3 Modeled temperature response profiles for 4-element model. .................................................. 92

Fig. 5.4 Thermal diagram of the two-element approximation of the microcalorimeter............................ 93

viii 
Fig. 5.5 Comparison of two-element and four-element models. ..................................................... 94

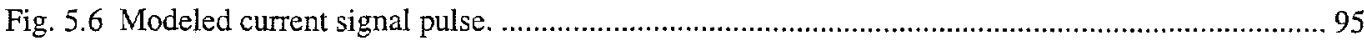

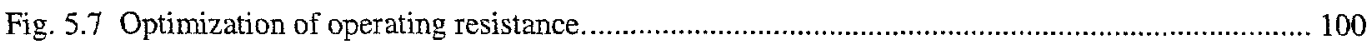

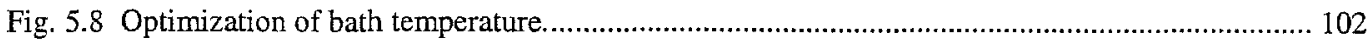

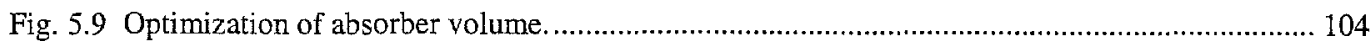

Fig. 5.10 Optimization of Stycast diameter. .............................................................................. 105

Fig. 5.11 Optimization of superconducting transition temperature ............................................. 106

Fig. 5.12 Optimization of superconducting transition width. ...................................................... 107

Fig. 5.13 Optimization of transition temperature for different bath temperatures.................................. 109

Fig. 5.14 Optimization of Stycast diameter for difference Stycast thicknesses. ..................................... 109

Fig. 6.1 Illustration of fabrication steps with cross sectional and top views. ..................................... 112

Fig. 6.2 TEM micrographs showing typical $\mathrm{Mo} / \mathrm{Cu}$ multilayer structures. ........................................... 114

Fig. 6.3 Optical micrograph showing the absorber supported by Stycast epoxy. ................................. 116

Fig. 6.4 a) Dc SQUID circuit with input and feedback coils. b) Dc SQUID current ys. applied field. .... 118

Fig. 6.5 Schematic of voltage bias circuit with parallel shunt and dc SQUID read-out. ....................... 120

Fig. 6.6 Schematic cross section of the adiabatic demagnetization refrigerator. .................................. 121

Fig. 6.7 Entropy of FAA salt pill for different applied magnetic fields............................................. 123

Fig. 6.8 Diagram of ground connections in typical experimental setup. ............................................. 127

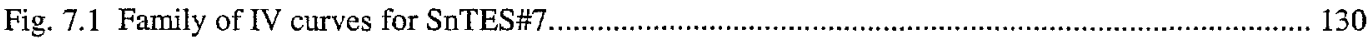

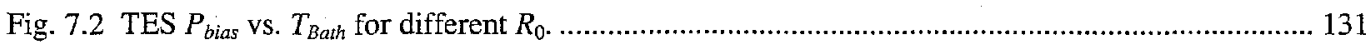

Fig. 7.3 SnTES\#7 IV curve fitted parameters.............................................................................. 132

Fig. 7.4 Thermal conductivity of SiN for SnTES\#7 .............................................................. 133

Fig. 7.5 Superconducting transition of the TES derived from IV analysis for SnTES\#7..................... 134

Fig. 7.6 Current dependence of the superconducting transition for SnTES\#7..................................... 136

Fig. 7.7 SnTES\#7 load line for constant external $V_{\text {Bias }}=197.7 \mathrm{mV}, R_{\text {Bias }}=2 \mathrm{k} \Omega, R_{\text {Shunt }}=10 \mathrm{~m} \Omega$......... 137

Fig. 7.8 SnTES\#7 transition along load line (black dots) compared with complete set (gray dots)......... 137

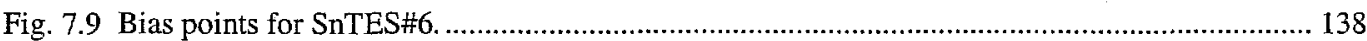

Fig. 7.10 Superconducting transition mapped out by bias points of SnTES \#6.................................... 140

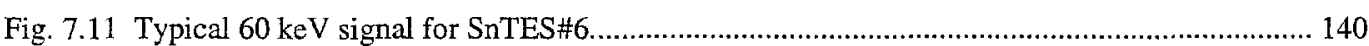

Fig. 7.12 Response of SnTES\#6 for constant resistance bias points. ............................................. 142

Fig. 7.13 Response of SnTES\#6 for constant $T_{\text {Bath }}$ bias points. ....................................................... 143

Fig. $7.1460 \mathrm{keV}$ spectral peak measured by SnTES\#6 with energy resolution of $340 \mathrm{eV}$.................... 144

Fig. 7.15 Noise spectrum of SnTES\#6 compared with theoretical noise contributions............................ 146

Fig. 7.16 Measured noise, theoretical noise, and pulse height at different bias points.......................... 148

Fig. 7.17 SnTES\#7 measured pulses for $8 \mathrm{keV}$ to $60 \mathrm{keV}$ energies. ............................................. 150

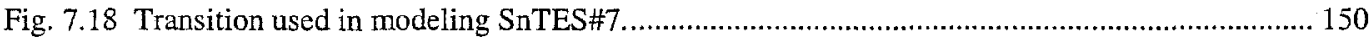

Fig. 7.19 SnTES\#7 measured (solid lines) and modeled (dotted lines) pulses for several energies......... 152 
Fig. 7.20 SnTES\#7 pulse heights and decay times for measured (dots) and modeled (line) pulses

Fig. 7.21 SnTES\#7 spectrum with energy resolution of $240 \mathrm{eV}$ at $60 \mathrm{keV}$ degraded by non-linear gain. 154

Fig. 7.22 Noise spectrum of SnTES\#7 compared with theoretical noise contributions. 155

Fig. 7.23 SnTES\#8 superconducting transition derived from IV curve at $T_{\text {Bath }}=65 \mathrm{mK} \ldots \ldots \ldots \ldots \ldots \ldots \ldots \ldots . . . . . . . .157$

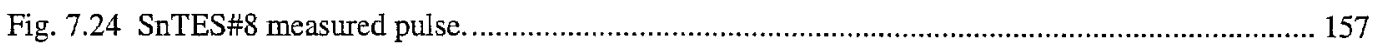

Fig. 7.25 SnTES\#8 pulse heights and decay times for measured (dots) and modeled (line) pulses......... 159

Fig. 7.26 Transition used in modeling SnTES\#8......................................................................... 159

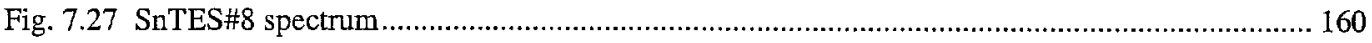

Fig. 7.28 SnTES\#8 Noise spectrum and calculated noise sources.................................................... 162

Fig. 8.1 Simulation of absorber and TES temperatures................................................................. 171

Fig. 8.2 Simulation of non-linear transition and energy response in voltage bias mode....................... 172

Fig. 8.3 Simulation of non-linear transition and energy response in active bias mode. ........................ 172

Fig. 8.4 Simulation of reduction in $\Delta T_{T E S}$ from voltage bias mode and active bias mode....................... 173

Fig. 8.5 a) Theoretical phonon noise spectra and b) Johnson noise spectra for varying values of $R_{c} \cdots \ldots . . .175$

Fig. 8.6 Summing element of active bias circuit. ........................................................................... 176

Fig. 8.7 Complete active negative feedback bias circuit. ......................................................... 177

Fig. 8.8 SnTES汭 decay times with different bias modes.............................................................. 179

Fig. 8.9 SnTES\#7 TES change in TES temperature with different bias modes..................................... 180

Fig. 8.10 SnTES\#7 pulse heights with different bias modes........................................................... 181

Fig. 8.11 SnTES\#7 $60 \mathrm{keV}$ pulse shapes with different bias modes. ............................................. 181

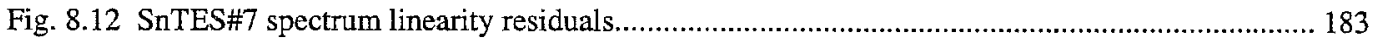

Fig. 8.13 SnTES\#7 spectra measured with different bias modes....................................................... 183 
Chapter 1

\section{BACKGROUND}

\subsection{Motivation}

The motivation for the project described in this dissertation stems from the need for a gamma-ray spectrometer with ultra-high energy resolution. This instrument has applications in nuclear non-proliferation and arms control programs within the Lawrence Livermore National Laboratory (LLNL) and the Department of Energy. Fissile materials with different intended purposes have different isotopic ratios. Fig. 1.1 shows the spectra from different levels of ${ }^{235} \mathrm{U}$ enrichment as measured by spectrometers with different energy resolutions. We see that the difference between $96 \%$ and $98 \%$ enrichment can only be resolved by a high energy resolution spectrometer. The low energy resolution spectra are comparable to commercially available spectrometers and are unable to distinguish between the two levels of enrichment. 


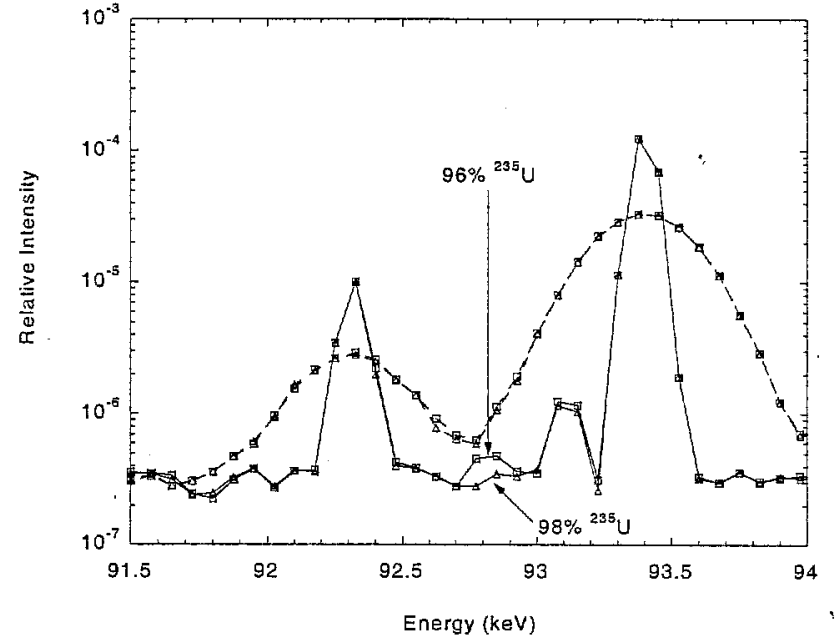

Fig. 1.1 Simulated spectra of $96 \%$ and $98 \%{ }^{235} \mathrm{U}$ enrichment measured with different energy resolution. Solid lines denote $100 \mathrm{eV}$ energy resolution response. Dashed lines denote $1 \mathrm{keV}$ energy resolution response. Triangles denote $98 \%{ }^{235} \mathrm{U}$ enrichment and squares denote $96 \%{ }^{235} \mathrm{U}$ enrichments.

A high energy resolution gamma-ray spectrometer will also be useful in environmental monitoring applications. Consider the detection of uranium against a background of radium. The most prominent gamma-ray line of ${ }^{235} \mathrm{U}$ is at $185.72 \mathrm{keV}$. However, ${ }^{226} \mathrm{Ra}$ has a gamma-ray line at $186.21 \mathrm{keV}$. Fig. 1.2 shows a simulated spectrum of small amounts of ${ }^{235} \mathrm{U}$ against a background of ${ }^{226} \mathrm{Ra}$. The two lines are unresolved with $1 \mathrm{keV}$ energy resolution, which is typical of commercially available spectrometers. However, with $100 \mathrm{eV}$ energy resolution, the lines are clearly separated. Thus, small traces of uranium in the environment are difficult to detect without the aid of a high energyresolution spectrometer. 


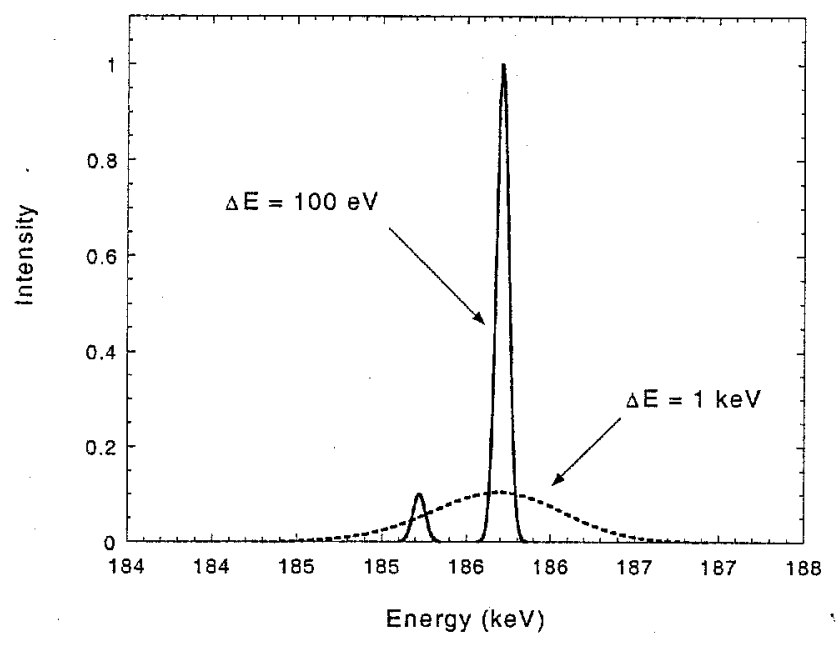

Fig. 1.2 Simulated spectrum of ${ }^{235} \mathrm{U}$ against ${ }^{226} \mathrm{Ra}$ background with different energy resolutions.

In astronomy, some of the most energetic phenomena emit gamma rays. High-resolution gamma-ray spectroscopy will enhance the measurements of nuclear emission lines such as ${ }^{56} \mathrm{Ni}$ at $158 \mathrm{keV}$ and ${ }^{44} \mathrm{Ti}$ at 68 and $78 \mathrm{keV}$. The lines are produced by material that has recently been ejected by supernovae, and can provide unique insight into the mechanism that creates and distributes heavy elements throughout the galaxy. Also, since most astronomical objects are very faint, the spectrometers must have good quantum efficiency as well as good energy resolution.

\section{$1.2 \quad$ Project history}

As early as 1935 , calorimeters have been cooled to low temperatures (50 $\mathrm{mK}$ ) to increase their sensitivity [1]. By 1975, it has been suggested that detection of single photons was possible [2]. In 1984 Mosely, Mather, and McCammon presented a theory for the fundamental energy resolution of microcalorimeters, demonstrating that single photon 
detection is possible [3]. McCammon, et al., obtained an energy resolution of $7.3 \mathrm{eV}$ for $6 \mathrm{keV} x$ rays using a silicon thermistor in 1993 [4]. However, this spectrometer suffered from a low count rate due to a pulse decay time constant of $\sim 10 \mathrm{~ms}$. Around the same time, LLNL developed superconducting tunnel junctions (STJ) capable of measuring 29 $\mathrm{eV}$ energy resolution for $6 \mathrm{keV} x$ rays [5] with a count rate several orders of magnitude greater than McCammon [6]. In 1995, Irwin developed a thorough theory for'noise in superconducting transition-edge sensors (TES) as bolometers [7][8]. By 1999, Irwin, et al., at the National Institute of Standards and Technology (NIST) used a TES to obtain an energy resolution of $4.5 \mathrm{eV}$ for $6 \mathrm{keV} \mathrm{x}$ rays, which is the best calorimetric measurement to date. For the last decade, LLNL has been actively developing advanced radiation detectors. We discuss some of our achievements in the following sections.

\subsection{Prior work at LLNL}

The Advanced Detector Group (ADG) at LLNL has been successful at the development of STJs for high energy resolution and high count rate detection of low energy $\mathrm{x}$ rays. In the last five years, the ADG has begun efforts to extend our detection capabilities into the high energy $x$ ray and gamma ray energy range. Our STJs are thin film devices which have poor absorption efficiency for photons above $1 \mathrm{keV}$, therefore, we require a new detector design.

\subsubsection{Tantalum crystals}

For gamma ray spectrometers, the absorber is of primary concern. Early in the project,

we developed techniques to build quasiparticle (excited electronic states in a 
superconductor) sensors on superconducting tantalum crystals. One of the advantages of this approach is that the number of quasiparticles produced during the absorption of a photon is independent of the volume of the absorber, thus allowing large crystals to be used as absorbers. Furthermore, since Ta has good stopping power for energetic photons, we can fabricate spectrometers that can absorb gamma rays with extremely high efficiency. The quasiparticle excitations and phonons must be able to diffuse through the bulk volume, thus requiring the Ta to be in a highly pure crystalline state. The Ta crystal must also have an extremely smooth surface for microfabrication of the sensor components. With mechanical and chemical polishing, we were able to obtain a surface roughness of $4 \AA$ rms over a $1 \mu \mathrm{m}^{2}$ area. The mean free path in a metallic material can be expressed in terms of its residual resistance ratio (RRR). At room temperatures, electrical resistance is dominated by electron-phonon scattering. At very low temperatures, electrical resistance is dominated by impurity scattering. RRR is defined as the ratio of electrical resistance at room temperature and low temperature, and the mean free path is given by

Eq. 1.1

$$
\lambda=R R R \frac{m_{e} \nu_{F}}{n e^{2} \rho_{300 K}},
$$

where $m_{e}$ is the electron mass, $v_{F}$ is the Fermi velocity, $n$ is the electron density, $e$ is the electron charge, and $\rho_{300 \mathrm{~K}}$ is the electrical resistivity at room temperature. In principle, with sufficiently pure Ta crystals, we can construct a spectrometer with an arbitrarily large absorber. This absorber may be coupled to either an STJ or a TES for signal readout. 


\subsubsection{Ta crystals with STJ sensors}

Our first design for a Ta-crystal based gamma-ray spectrometer was codenamed "Luxor". A Luxor device consists a thin piece of bulk Ta crystal diffusion-bonded at high temperature and high pressure to a sapphire substrate. The STJ sensor is microfabricated on top of the Ta. From the Ta crystal to the base and top electrodes of the STJ, each layer was successively smaller, similar to a pyramid structure. Experiments with this detector design yielded no measured signals from photons absorbed in the Ta. Only signals from soft $x$ rays were observed via direct absorption in the STJ films [9].

\subsubsection{Ta crystals with TES}

We decided to pursue TES-based spectrometers because the quality of an STJ is highly dependent on the surface roughness of the underlying layers. The quality of a TES is less susceptible to surface roughness. Furthermore, TES sense thermal energy, which is the ultimate fate of all excitations created by incident gamma rays. STJs can only sense quasiparticles and athermal phonons, which limit their sensitivity. The next detector design was a bulk, free-standing, piece of Ta crystal with a TES microfabricated on the surface. Experiments with this detector design only yielded signals from absorption directly under the TES film. No other absorption events were observed [9].

\subsubsection{InSbTES}

It has been noted that $\mathrm{Ta}$ has slower than expected quasiparticle diffusion and shorter than expected quasiparticle lifetimes (as predicted by thermal recombination) [10][11]. 
This prompted us to explore using dielectric crystals instead of Ta. TESs were fabricated on top of a free-standing piece of bulk crystalline $\mathrm{InSb}$. The best energy resolution measured with this device is $1.4 \mathrm{keV}$ for $60 \mathrm{keV}$ gamma rays [9] with strong position dependence.

\subsubsection{X-ray spectrometers}

Concurrently, we also pursued TES-based x-ray spectrometers. The design exploited the low thermal conductivity of silicon nitride (SiN) membranes on Si substrates. A thin film ( $3 \mu \mathrm{m}) \mathrm{Cu}$ absorber is deposited on the membrane. The TES film forms a bridge between the thermally isolated $\mathrm{Cu}$ absorber and the $\mathrm{Si}$ substrate, which serves as a cold bath. Experiments with this device yielded an energy resolution of $42 \mathrm{eV}$ for $6 \mathrm{keV} x$ rays [12]. In addition, we further extended the theory of Irwin and developed a comprehensive model for microcalorimeters that include temperature and current dependence [12].

\subsection{Current work at LLNL}

\subsubsection{Bulk absorber coupled to TES}

At present, there are several microcalorimeter projects at LLNL. Among them is the development of the high energy resolution gamma-ray spectrometer, which is the topic of this dissertation. By using a bulk Sn absorber coupled to a TES film isolated on a SiN membrane (Chapter 4, page 71), we were able to measure an energy resolution of $70 \mathrm{eV}$ for $60 \mathrm{keV}$ gamma rays, which is among the best measurements of its kind to date (Section 7.10, page 162). Despite this achievement, we noted weaknesses in the 
performance of the detector and addressed them by implementing an active negative feedback bias technique (Chapter 8, page 167).

\subsubsection{Absorber study}

To further improve our successful detector design, we are studying the performance of different materials as absorbers [13]. A better choice of material can significantly improve the absorption efficiency and heat capacity. Some of the absorbers studied include $\mathrm{Mo}, \mathrm{Re}, \mathrm{Pb}$, and $\mathrm{Ta}$. Note that the Ta absorber is implemented in the current design, not the previous designs.

\subsubsection{Fabrication control}

Even though we achieved a good energy resolution, our fabrication processes need improvement. With our current fabrication technique, we have low control and repeatability over the thermal properties of the microcalorimeter. Efforts are underway to develop better processing techniques, such as a full wafer etch process for the TES films (instead of photographically defined lift-off) and microfluidic dispensing systems for deposition of epoxy to couple the absorber to the TES. Other methods of adhering the absorber are also being considered, such as indium bump bonding.

\subsubsection{Ac bias}

Among the many goals for the ADG is the development of detector arrays. Device bias and signal read out of an array can be made by duplicating the electronic components for each pixel, which is costly and inefficient. An alternative method is to multiplex in the 
frequency domain by ac biasing each TES pixel with a unique frequency [14][15]. Efforts are underway to develop multiplexing schemes.

\subsection{Concurrent work elsewhere}

\subsubsection{Bulk absorbers coupled to semiconducting thermistors}

There are similar gamma-ray spectrometer projects in institutions around the world. A prominent group is lead by Egelhof at Gesellschaft für Schwerionenforschung in Germany. They have had success developing devices using bulk absorbers coupled to neutron transmutation doped (NTD) Ge thermistors [16] (Section 7.10, page 162).

\subsubsection{Magnetic calorimeter}

Most spectrometer designs have temperature-dependent resistive sensors. At Universität Heidelberg in Germany, Seidel, et al., developed a magnetic calorimeter [17][18][19]. The basic principle is that the magnetization of a paramagnetic material is dependent on temperature. The change in magnetization is sensed by a SQUID input coil. They were able to measure an energy resolution of $120 \mathrm{eV}$ for gamma rays up to $200 \mathrm{keV}$ with less than $1 \%$ deviation from linear response.

\subsection{Future work at LLNL}

The microcalorimeter project at LLNL has demonstrated good results and shown great potential. The next step in the research process is fabrication control. This allows further characterization and study of our devices. Development of arrays is also important, as it increases total count rate and opens up the possibilities of imaging applications. 


\section{References}

[1] F. Simon, Nature, 135, 673 (1935).

[2] T.O. Ninikoski, Liquid and Solid Helium, Halsted, New York (1975).

[3] S.H. Moseley, J.C. Mather, D. McCammon, J. Appl. Phys., 56 (5), 1257 (1984).

[4] D. McCammon, W. Cui, M. Juda, J. Morgenthaler, J. Zhang, Nucl. Instr. 'Meth. A, 326, 157 (1993).

[5] M. Frank, C.A. Mears, S.E. Labov, F. Azgui, M.A. Lindemạ, L.J. Hiller, H. Netel, A. Barfknecht, Nucl. Instr. Meth. A., 370, 41 (1996).

[6] M. Frank, L.J. Hiller, J.B. le Grand, C.A. Mears, S.E. Labov, M.A. Lindeman, H. Netel, D. Chow, Rev. Sci. Instrum., 69, 25 (1998).

[7] K.D. Irwin, Ph.D. Dissertation, Stanford University (1995).

[8] K.D. Irwin, Appl. Phys. Lett., 66(15), 1998 (1995).

[9] H. Netel, Ph.D. Dissertation, Universiteit Twente (1999).

[10] M. van den Berg, Ph.D. Dissertation, Universiteit Utrecht (1999).

[11] S. Friedrich, K. Segall, M.C. Gaidis, C.M. Wilson, D.E. Prober, A.E. Szymkowiak, S.H. Moseley, Appl. Phys. Lett., 71, 3901 (1997).

[12] M.A. Lindeman, Ph.D. Dissertation, University of California, Davis (2000).

[13] M.L. van den Berg, D.T. Chow, A. Loshak, M.F. Cunningham, T.W. Barbee, Jr., M. Frank, S.E. Labov, Proc. SPIE, 4140, 436 (2000).

[14] T. Miyazaki, Ph.D. Dissertation, University of Tokyo (2001).

[15] J. Yoon, et al., Appl. Phys. Lett., 78, 371 (2001). 
[16] A. Bleile, P. Egelhof, H.-J. Kluge, U. Liebisch, D. McCammon, H.J. Meier, O. Sebastián, C.K. Stahle, M. Weber, Nucl. Instr. Meth. A, 444, 488 (2000).

[17] A. Fleischmann, C. Enss, J. Schönefeld, J. Sollner, K. Horst, J.S. Adams, Y.H. Kim, G.M. Seidel, S.R. Bandler, Nucl. Instr. Meth. A, 444, 100 (2000).

[18] J. Schönefeld, C. Enss, A. Fleischmann, J. Sollner, K. Horst, J.S. Adams, Y.H. Kim, G.M. Seidel, S.R. Bandler, Nucl. Instr. Meth. A, 444, 211 (2000).

[19] C. Enss, A. Fleischmann, K. Horst, J. Schonefeld, J. Sollner, J.S. Adams, Y.H. Huang, Y.H. Kim, G.M. Seidel, Journ. Low Temp. Phys., 121, 137 (2000). 
Chapter 2

\section{INTRODUCTION TO}

\section{ENERgy Dispersive Detectors}

\subsection{Energy-dispersive detectors}

In the context of this dissertation, a detector is a device capable of measuring energetic particles and/or radiation. A detector with the ability to distinguish between different energies of the incident particles and/or radiation are known as energy-dispersive detectors.

The detection process requires several steps. These steps are: absorption, excitation, measurement, and recovery to equilibrium. Each of these steps is described in detail in the following sections. Since the primary interest lies in the detection of gamma-rays, the emphasis will be placed on detection of photons with energy greater than $10 \mathrm{keV}$. 


\subsection{Absorption processes}

For radiation to be measured, it must first be absorbed in the detector. Absorption of the photon transfers the energy of the photon into the detector. The interactions of gammaray photons with matter are described by three primary processes [1]. These processes are photoelectric absorption, Compton scattering, and pair production, all shown in Fig. 2.1 .
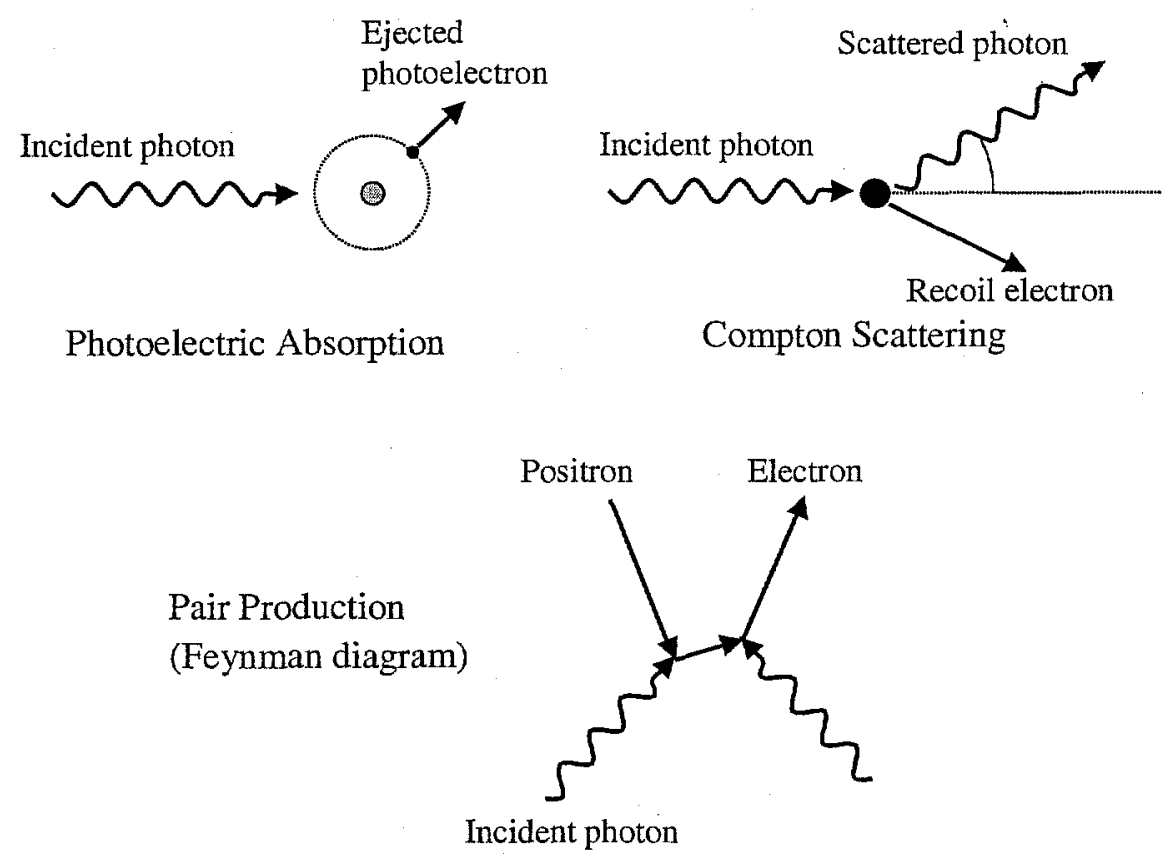

Fig. 2.1 Gamma-rays interactions with matter.

\subsubsection{Photoelectric absorption}

In photoelectric absorption, the incident photon is absorbed completely by an atom. The atom then ejects an electron from one of its shells. This electron is known as a photoelectron. The photoelectron leaves the atom with a kinetic energy of 


$$
E_{e}=h v-E_{b}
$$

where $E_{b}$ is the binding energy of the electron in the shell it originated from, $h$ is Planck's constant, and $v$ is the frequency of the photon. Typically, the photoelectron is ejected from an inner shell, such as the $\mathrm{K}$ shell. The ejection of a photoelectron leaves the atom in an ionized and excited state with a vacancy in an inner orbital. The atom quickly fills the hole by capturing a free electron or rearrangement of its orbital electrons. This leads to the emission of characteristic $\mathrm{x}$-ray photons. If the vacancy is in the $\mathrm{K}$ shell and an $\mathrm{L}$ shell electron fills it, the characteristic $\mathrm{x}$-ray photon will have an energy equal to the $\mathrm{K} \alpha$ transition in the atom. In some cases, the excited atom may release its energy in the form of an Auger electron. The Auger electron will similarly possess the characteristic $\mathrm{K} \alpha$ energy. Fig. 2.2 illustrates the processes that follow photoelectric absorption.

The photoelectron, characteristic $\mathrm{x}$-ray, and Auger electron generally travel only a short distance before encountering another interaction. Therefore, the full energy of the incident photon is usually completely absorbed in small volume. However, if the photoelectron, characteristic x-ray, or Auger electron escapes from the absorber volume, then only a portion of the energy of the incident photon is absorbed. The subsequent measured signal will misrepresent the true energy of the incident photon. The measured spectrum will then have additional features. Spectral features are discussed in detail in Section 2.7 on page 33. For $x$ rays and gamma rays of relatively low energy $(<100 \mathrm{keV})$, photoelectric absorption is the dominant interaction process. 


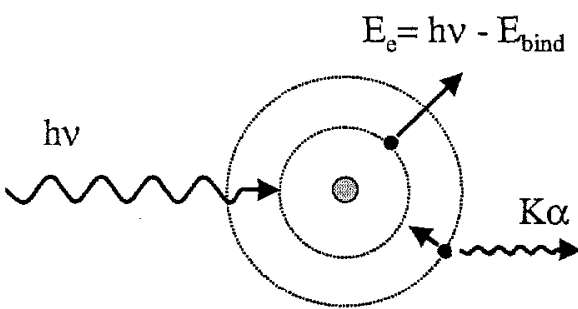

a)

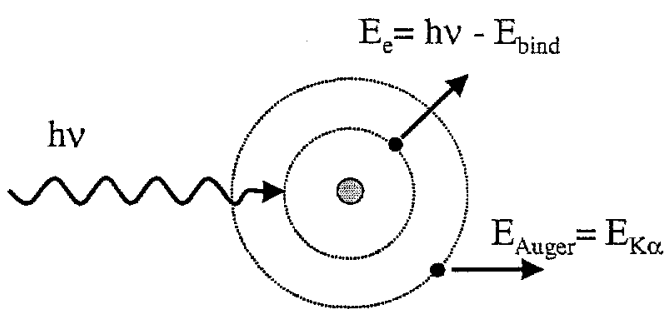

b)

Fig. 2.2 Example of processes following photoelectric absorption.

a) Photoelectric absorption followed by emission of characteristic x-ray. b) Photoelectric absorption followed by emission of Auger electron.

The probability of absorption depends on the atomic number $Z$ of the absorber, and the energy $h v$ of the photon. Intuitively, we can expect higher absorption probability with "heavier" elements. Similarly, we also expect energetic photons to be more penetrating. No single analytic expression is valid for the probability of photoelectric absorption over all ranges of $E$ and $Z$. Experimentally, it can be described by

Eq. 2.2

$$
\frac{I}{I_{0}}=\exp \left(-\frac{\mu}{\rho} \rho t\right)
$$

where $I_{0}$ is the intensity of the incident beam of photons, $I$ is the intensity of the beam after passing through an absorbing material, $\rho$ is the density of the material, and $t$ is the thickness of the material. The parameter $\rho t$ is known as the mass thickness, and $\mu \rho$ is the attenuation coefficient. The attenuation coefficients for most elements have been measured experimentally and found to approximately follow the relation [2] 


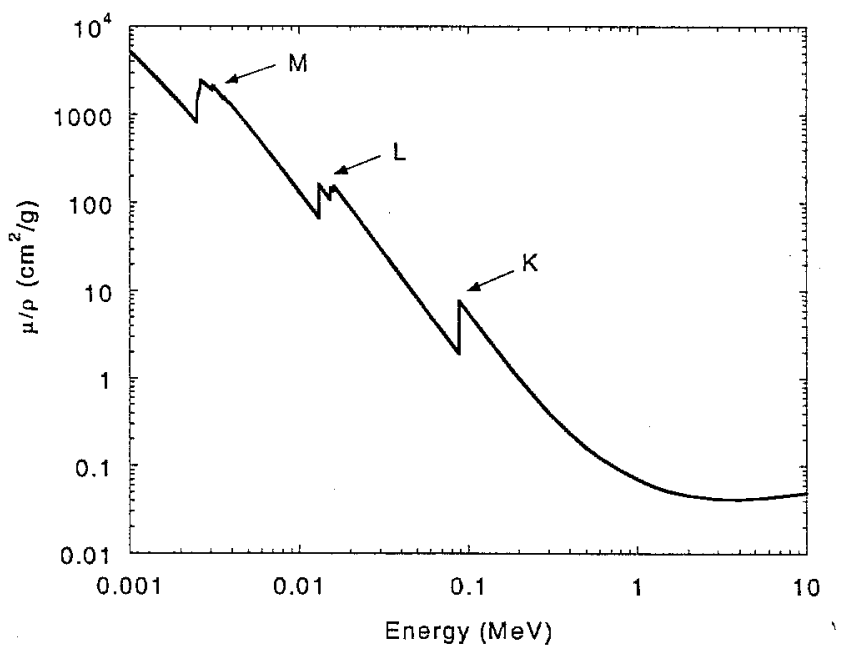

Fig. 2.3 Attenuation coefficients for $\mathrm{Pb}$.

Also indicated are the $\mathrm{M}, \mathrm{L}$, and $\mathrm{K}$ absorption edges. The structure at the top of the $\mathrm{L}$ edge corresponds to the absorption edges of the L1, L2, and L3 transitions. The structure at the top of the M edge corresponds to the absorption edges of the M1, M2, M3, M4, and M5 transitions.

Eq. 2.3

$$
\frac{\mu}{\rho} \propto \frac{Z^{4}}{E^{3}}
$$

Because of the strong dependence on $Z$, it is preferable to use a high $Z$ material such as $\mathrm{Pb}$ as a radiation absorber. A plot of the attenuation coefficient for $\mathrm{Pb}$ is shown in Fig. 2.3 .

For any element, the absorption efficiency generally decreases with increasing energy. However, there are discontinuities known as absorption edges. These absorption edges correspond to the electron transitions allowed for a particular element. For example, consider an atom with $\mathrm{K}$ and $\mathrm{L}$ shells. If the incident photon energy is less than the energy necessary for excitations in the $\mathrm{K}$-shell, then the photon can only be absorbed by 
excitation of the L-shell electrons. However, if the photon energy is above the K-shell excitation energy, then it can be absorbed by excitation of K-shell electrons as well as the L-shell electrons, thus causing a discontinuous increase in the absorption efficiency. The structure at the top of an absorption edge corresponds to absorption by electrons at different levels with transitions originating from that shell, such as L1, L2, and L3 for the L-shell transitions.

\subsubsection{Compton scattering}

For photons of higher energy (typically $>100 \mathrm{keV}$ ), Compton scattering becomes significant. Compton scattering occurs when an incident photon elastically scatters off an electron initially at rest. The photon imparts some of its energy to the electron, causing it to recoil. The scattered photon also leaves the interaction in a different direction with less energy. Fig. 2.4 illustrates the effect of Compton scattering. For an incident photon with energy $h v$, the scattered photon will have energy $h v^{\prime}$ given by [2]

$\mathrm{Eq} .2 .4$

$$
h v^{\prime}=\frac{h v}{1+\left(h v / m_{e} c^{2}\right)(1-\cos \theta)}
$$

where $m_{e}$ is the electron rest mass and $\theta$ is the angle by which the photon scattered. By conservation of energy, the kinetic energy of the recoil electron is

Eq. 2.5

$$
E_{e}=h v-h v^{\prime}
$$




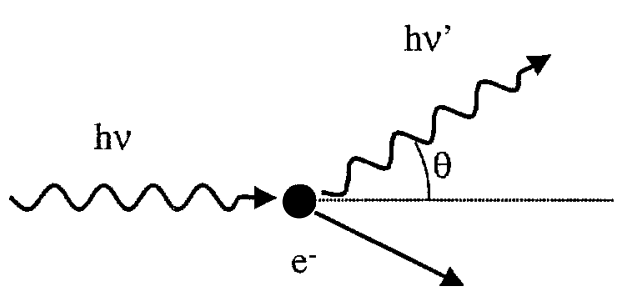

Compton scattering

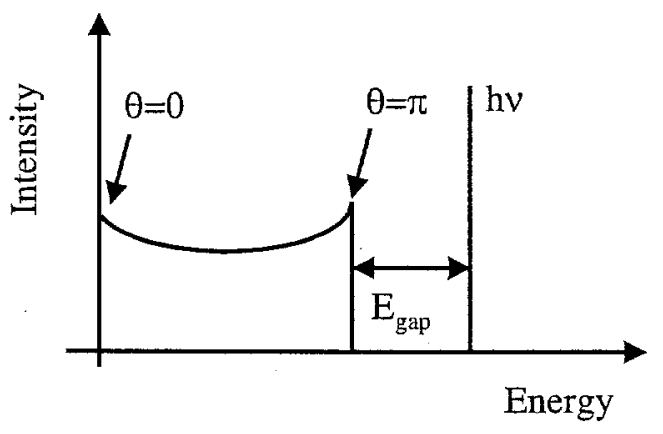

Compton continuum

Fig. 2.4 Compton scattering and Compton continuum.

Two interesting situations occur when the photon scatters at a grazing angle $(\theta \cong 0)$, and when the photon complete backscatters $(\theta=\pi)$. For scattering at grazing incidence, the photon energy does not change significantly $\left(h v \cong h v^{\prime}\right)$. The energy transferred to the electron is near zero. For complete backscatter, the energy transferred to the electron is

Eq. 2.6

$$
\left.E_{e}\right|_{\theta=\pi}=h v\left(\frac{2 h v / m_{e} c^{2}}{1+2 h v / m_{e} c^{2}}\right),
$$

which is less than $h v$. If the scattered photon escapes from the absorber volume, then in the spectrum there will be a continuum of measured energies corresponding to the energy deposited into the absorber at different scattering angles. This is known as the Compton continuum (Fig. 2.4), and ranges from zero energy for grazing incidence to the energy absorbed by an electron from complete backscatter. There is a gap between the top of the continuum and the full energy line of the photon. This Compton gap is given by 
Eq. 2.7

$$
E_{G a p}=h v-\left.E_{e}\right|_{\theta=\pi} .
$$

Spectral features from Compton scattering are discussed in Section 2.7 on page 33. The cross section for Compton scattering depends on the atomic number $Z$ of the absorbing material and the energy of the incident photon $h v$. The differential cross section is given by the Klein-Nishina formula

Eq. $2.8 \frac{d \sigma}{d \Omega}=Z r_{0}^{2}\left(\frac{1}{1+\left(\frac{h v}{m_{e} c^{2}}\right)(1-\cos \theta)}\right)^{2}\left(\frac{1+\cos ^{2} \theta}{2}\right)\left(1+\frac{\left(\frac{h v}{m_{e} c^{2}}\right)^{2}(1-\cos \theta)^{2}}{\left(1+\cos ^{2} \theta\right)\left(1+\left(\frac{h \nu}{m_{e} c^{2}}\right)(1-\cos \theta)\right)}\right)$

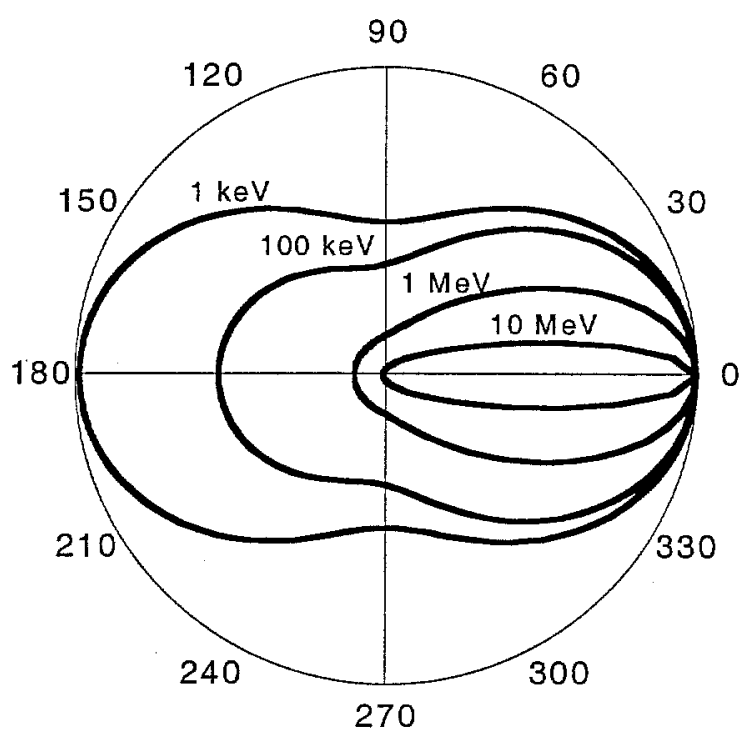

Fig. 2.5 Angular cross section of Compton scattering. 
where $r_{0}$ is the classical electron radius. Fig. 2.5 shows the differential cross section as a function of scattering angle for various photon energies. Note that as energy increases, the probability for forward scattering increases.

\subsubsection{Pair production}

At very high energies, gamma rays can interact with matter in a process knowh as pair production. In this process, the photon vanishes and creates an electron-position pair. Therefore, the minimum energy necessary for this process to occur is two electron rest masses $(2 \times 511 \mathrm{keV})$. Pair production is best illustrated by a Feynman diagram (Fig. 2.6).

The incident gamma-ray creates an electron-positron pair. However, in order to conserve momentum, one of the particles must exchange a virtual photon with an absorber atom. Pair production cannot happen in free space with only one photon. The positron is intrinsically unstable and will only travel a very short distance before it annihilates with an electron. This annihilation will create two gamma rays, each with an energy of 511 $\mathrm{keV}$. These two annihilation photons can interact with the absorbing material by Compton scattering and/or photoelectric absorption. If either or both of these annihilation photons escape from the absorber volume, the energy deposited into the absorber will be less than the energy of the incident photon. This causes escape lines, similar to the escape of characteristic $\mathrm{x}$ rays in photoelectric absorption. Escape lines and other spectral features are discussed in Section 2.7 on page 33. 


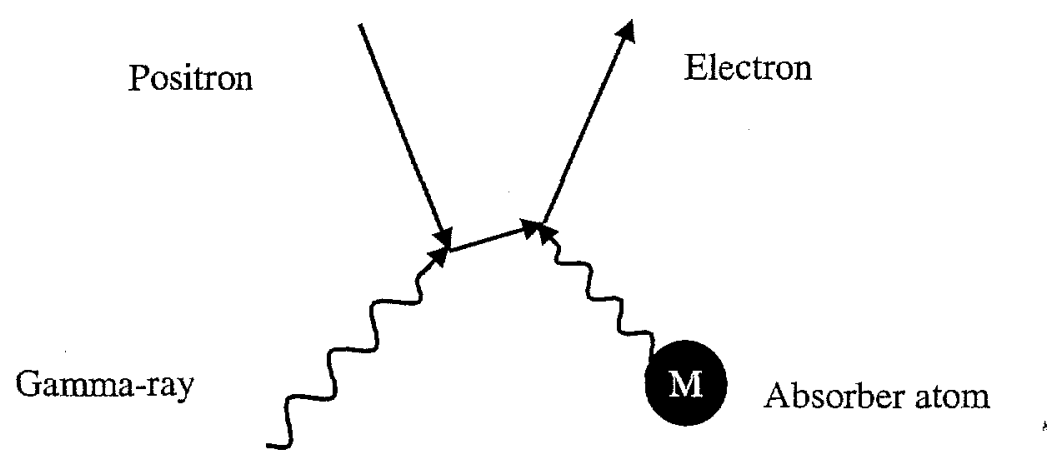

Fig. 2.6 Feynman diagram illustrating pair production.

The energy of the incident gamma ray is deposited into the absorber through combinations of photoelectric absorption, Compton scattering, and pair production. If no secondary photons escape from the absorbing volume, then the total energy deposited is equal to the energy of the incident photon. If secondary photons escape from the absorbing volume, then the energy deposited is equal to the energy of the incident photon minus the energy of the escaped photons.

\subsection{Excitations}

An excitation is an energy state above the ground state. Thermal energy can create excitations. Once energy is deposited into an absorber, additional excitations are created. The types of possible excitations are material dependent. These initial excitations are athermal. For a semiconductor, the excitations are electron-hole pairs. For a superconductor, they are broken Cooper pairs known as quasiparticles. For a crystalline material, they are athermal phonons. For all materials, some of the excitation energy is in the form of phonons. The athermal excitations diffuse throughout the absorber volume. 
As they scatter, they create a cascade of less energetic excitations. Eventually, most athermal excitations will be converted to thermal phonons or heat.

\subsection{Signal readout}

Once the photon energy is absorbed and excitations are created, they can be measured. Generally, measurement of a signal requires a bias voltage (or current). The excitations typically cause a change in the electrical properties of the material such that the current (or voltage) will be altered. For example, in a semiconducting ionization detector, the detector is biased with an electric field. The steady state current depends on the number of existing electron-hole pairs. The absorption of a photon creates many additional electron-hole pairs. These serve as additional charge carriers and increase the current flowing through the detector. The change in current is proportional to the number of electron-hole pairs created, which in turn is proportional to the photon energy. In a superconducting tunnel junction, the quasiparticle excitations are analogous to electronhole pairs, acting as additional charge carriers and changing the resistivity of the detector. Since the ultimate fate of most excitations is thermal heating of the detector, there is a class of detectors that exploit this characteristic. Microcalorimeters measure the energy of an incident photon by the amount of heat deposited by the photon. For microcalorimeters, the detector material typically has a temperature-dependent resistivity such that a change in temperature causes a change in resistivity that can be measure with an appropriate current or voltage bias. 
For all energy-dispersive detectors, it is important that there is a correlation between the energy absorbed and the number of excitations created, and between the number of excitations and the signal size. With these correlations, it is possible to calibrate the detector response with energy.

\subsection{Equilibrium}

After measuring a signal that is proportional to the incident photon energy, the detector must be allowed to return to equilibrium. For semiconductor detectors; the electron-hole pairs will recombine at the electrodes. In a superconducting tunnel junction, the quasiparticles will recombine into Cooper pairs with time. For a microcalorimeter, there must be a thermal link between the absorbing material and a heat reservoir so that excess heat is allowed to flow out of the device. It is crucial that the thermal link be sufficiently weak such that the detector can thermalize and the signal measured before returning to equilibrium. Since all detectors suffer some amount of heating from downconversion of athermal excitations, the thermal link is necessary in all detectors. The strength of the thermal coupling varies among different types of detectors, depending on the detector's sensitivity to temperature. For some detectors, the gain or size of the measured signal depends strongly on the steady-state conditions. These conditions may include the operating temperature of the detector, the voltage or current bias, among others. For these detectors, it is crucially important that the detector be allowed to return to equilibrium before another absorption event. The time required to recover equilibrium is known as the "dead time." The necessity of dead time may affect aspects of the detector's performance such as count rate. 


\subsection{Spectrum and linewidth}

For energy-dispersive detectors, the terms "spectrometer" and "detector" both describe the same device. The distinction comes from the application of the device. Whereas a detector measures an absorption event, a spectrometer measures many absorption events

to produce a spectrum. Recall that the detector signal is correlated to the absorbed energy. A spectrum is a histogram of typically thousands or even millions of signals from energy absorption events. The horizontal axis of a spectrum is the energy of the event. The vertical axis is the number of events per energy bin (Fig. 2.7).

Every measurement includes some noise. Noise causes a fluctuation in the size of the signal. If there were no noise at all, all measurements of the same energy would give exactly the same signal. The spectrum would show an infinitely sharp peak with no spread in measured energy. Because of noise, measurements of the same energy will give a distribution of signal sizes that correspond to some range in energy. The spectrum will show a peak with a finite width in energy. The shape of the peak is Gaussian. The centroid of the peak is the energy of the absorption event. The width of the peak corresponds to the energy resolution of the detector and measurement process. The energy resolution is typically expressed by the full width at half-maximum (FWHM) of the peak. In terms of the Gaussian shape, FWHM is approximately $2.35 \sigma$, where $\sigma$ is the standard deviation. 


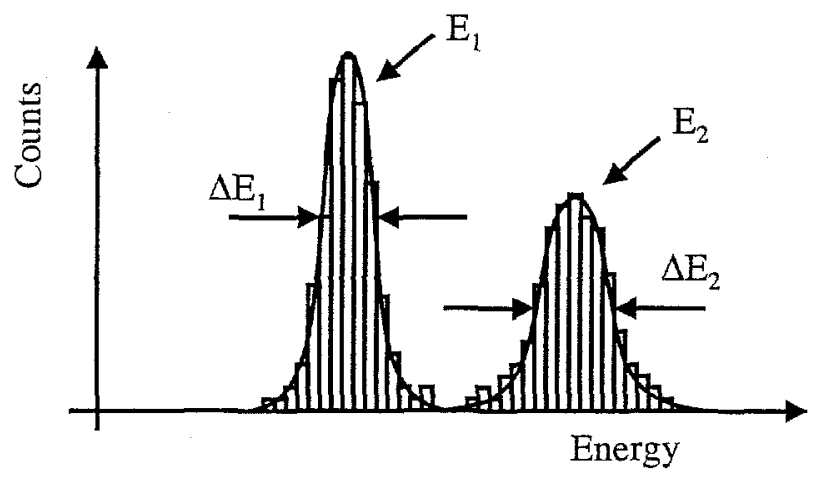

Fig. 2.7 Illustration of an energy spectrum.

The $\mathrm{x}$-axis represents the energy of each absorption event sorted into bins of constant energy. The $\mathrm{y}$-axis indicate the number of counts per bin. The energy at the centroid of each peak is denoted by $E_{1}$ and $E_{2}$. The width of each peak is denoted by $\Delta \mathrm{E}_{1}$ and $\Delta \mathrm{E}_{2}$.

Spectral resolving power refers to the ratio of energy to energy resolution $(E / \Delta E)$. High resolving power is sometimes called high energy resolution, and corresponds to peaks that are "narrow". Similarly, low resolving power is sometimes called low energy resolution and corresponds to peaks that are more "broad". If the incident photons were of two different energies, the spectrum will show two peaks. The area of each peak is the total number of absorption events at that energy. If the peaks have similar linewidths, the relative heights of the peaks are proportional to the relative intensities.

For most spectrometry applications, it is desirable to have high energy resolution. All phenomenon that contribute to the degradation of energy resolution in the detector are collectively known as noise. 


\subsubsection{Fluctuations}

In most detectors, the gain of the signal depends on bias parameters. The fluctuation of any of these parameters adds noise to the measurement. Some fluctuations are intrinsic and unavoidable. All resistive elements in the bias and read-out circuits of the detector have intrinsic Johnson noise. Most detector signals are electrical, thus making them prone to Johnson noise. All thermal links have intrinsic thermal fluctuations or phonon noise. Johnson noise and phonon noise are discussed in detail in Section 3.5 on page 52.

\subsubsection{Statistics}

For athermal detectors with signals that depend on the measurement of the number of excitations created, statistical noise plays an important role. The distribution of excitations created can be approximated by a Poissonian distribution. The Poisson distribution is given by [1]

Eq. 2.9

$$
P(x)=\frac{(\bar{x})^{x} e^{-\bar{x}}}{x !},
$$

where $x$ is the number of successes and $\bar{x}$ is the mean value of the distribution. The variance of the distribution is

Eq. 2.10

$$
\sigma=\sqrt{\bar{x}}
$$

The response of most detectors is linear, such that the number of excitations created is proportional to the energy of the photon. Therefore, the signal size is $E=K N$, where $N$ is 
the number of excitations and $K$ is a proportionality constant. The variance of the energy has a statistical limit given by $\Delta E=K \sqrt{N}$, giving an energy resolution of

Eq. 2.11

$$
\frac{\Delta E}{E}=\frac{2.35 K \sqrt{N}}{K N}=\frac{2.35}{\sqrt{N}}
$$

This is an important consideration in the design of a detector. For a photon of a given energy, materials with a lower energy threshold per excitation will have more excitations created. A large number of excitations will lead to a smaller statistical variance in the measurement. Superconducting tunnel junctions exploit this statistical phenomenon. Elemental superconductors typically have an excitation energy on the order of $1 \mathrm{meV}$. Semiconductors typically have an excitation energy of approximately $1 \mathrm{eV}$. The thousand-fold difference in the excitation energy means that a photon will produce a thousand times more excitations in a superconductor than in a semiconductor, thus significantly increasing the energy resolution by reducing the statistical variance. For a microcalorimeter, the largest quanta of thermal energy is a phonon with the Debye energy, typically $\sim 10 \mathrm{meV}$.

However, the distribution of excitations created will only be Poissonian if the creation of each excitation is an independent process. Since the creation of the excitations all result from the absorption of a single photon, they are not truly independent. Careful measurements of energy resolution in semiconductor detectors have found a statistical 
variance as much as ten times smaller than predicted by simple Poisson statistics. This difference is described by the Fano factor, given by [1]

Eq. 2.12

$$
F=\frac{\text { observed variance in } N}{\text { Poisson predicted variance }(=N)}
$$

The Fano limit of the energy resolution is then

Eq. 2.13

$$
\frac{\Delta E}{E}=\frac{2.35 K \sqrt{F N}}{K N}=2.35 \sqrt{\frac{F}{N}}
$$

For semiconductor ionization detectors such as $\mathrm{Si}$ and $\mathrm{Ge}$, the Fano factor is approximately $\sim 0.1$. Superconducting materials such as $\mathrm{Nb}$ have a Fano factor approximately $\sim 0.2$.

\subsubsection{Pile-up}

The arrival of incident photons is random. There is an average rate at which photons arrive. Because of the randomness of the process, some arrive sooner than the average interval, and some arrive later. Consider a detector with a signal amplitude that is proportional to energy and decays exponentially with time back to the equilibrium state. If another photon is absorbed during the decay "tail", the signal amplitude of this photon will be offset by the height of the tail at the moment of absorption. This amplitude will appear larger than it should be and misrepresent the energy of the photon. Fig. 2.9 shows an illustration of pile-up and its effect on the spectrum. 


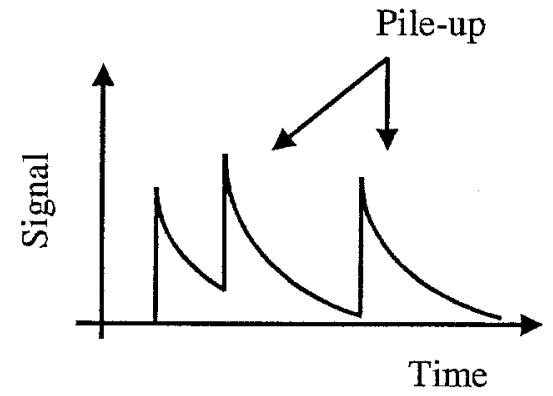

a)

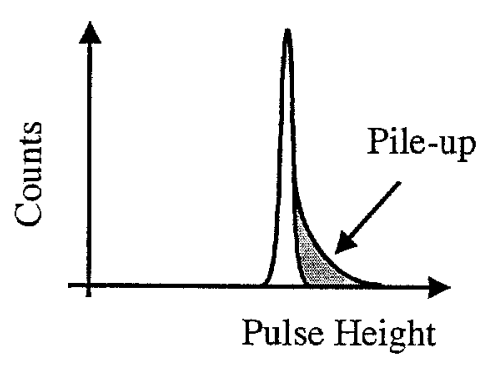

b)

Fig. 2.8 Schematic of pile-up and pile-up effect on spectrum.

a) Pile-up occurs when the detector absorbs a photon, then absorbs another photon before it recovers to equilibrium after absorption of the first photon. b) Pile-up events appear mostly on the high energy side of a peak. Severe pile-up will broaden the peak, decreasing energy resolution.

The distribution of photon arrival rates is Poissonian. For count rate considerations, $x$ is the number of events in a time window $t, \bar{x}=n t$ is the average number of events in the same time window, and $n$ is the average count rate. The Poisson distribution for the probability of $x$ events happening in time $\tau$ following a true event is given by

Eq. 2.14

$$
P(x)=\frac{(n t)^{x} e^{-n t}}{x !}
$$

A plot of this distribution is shown in Fig. 2.9. The probability of no pile-up events occurring $P(0)$ decreases exponentially as either count rate or measurement time window increases. 


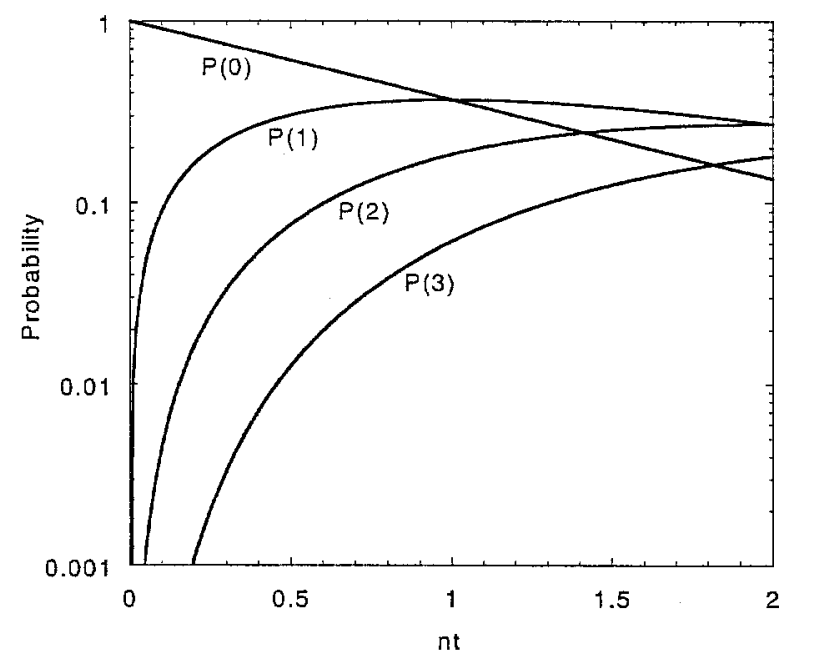

Fig. 2.9 Poisson distribution for pile-up events.

The probabilities for $0,1,2$, and 3 pile-up events are shown as a function $n t$, the product of count rate and measurement time window.

We can calculate the broadening of a monoenergetic spectral line due to pile-up events. We assume the pile-up of only two pulses. The pile-up of three or more pulses is unlikely except at extremely high count rates where $n t>1$. For exponential pulses, the amplitude of a pile-up of two pulses is

Eq. 2.15

$$
E=E_{0}+E_{0} e^{-\mathrm{t} / \tau}
$$

where $E_{0}$ is the amplitude of a pulse free of pile-up, and $t$ is the time at which the pile-up occurs. The decay time constant of the pulse is $\tau$. The value of $E$ ranges from $E_{0}$ for a pulse free of pile-up, to $2 E_{0}$ for the extreme case of two pulses arriving simultaneously. The probability of a pile-up pulse occurring in an interval $d \tau$ after a time $\tau$ is given by 
Eq. 2.16

$$
P_{p i l e-u p}(t) d t=n e^{-n t} d t
$$

Knowing the probability of pile-up as a function of time and the corresponding apparent amplitude due to such a pile-up, we can equate

Eq. 2.17

$$
P_{\text {pile-up }}(E) d E=P_{\text {pile-up }}(t) d t
$$

to find the normalized probability of pile-up per unit energy, which is

Eq. 2.18

$$
P_{\text {pile-up }}(E)=\frac{n \tau}{E_{0}}\left(\frac{E-E_{0}}{E_{0}}\right)^{n x-1} \text {, for } E_{0}<E<2 E_{0}
$$

This distribution is the broadening of a monoenergetic spectral line due to pile-up events. For detectors with finite resolution, the line broadening is the convolution of the pile-up energy distribution with the detector line shape in the absence of pile-up. Fig. 2.10 shows the broadening of a gaussian spectral peak due to pile-up events. In this example, the apparent increase in line width due to pile-up was determined by fitting a gaussian to the convolved pile-up energy distribution.

The above analysis is valid for detector systems that uses a constant baseline for all pulses measured. However, many detector systems measure the signals with triggered time windows. The baseline is determined for each and every signal. In this case, only pile-up events that fall within the measuring time window will affect the measurement. 

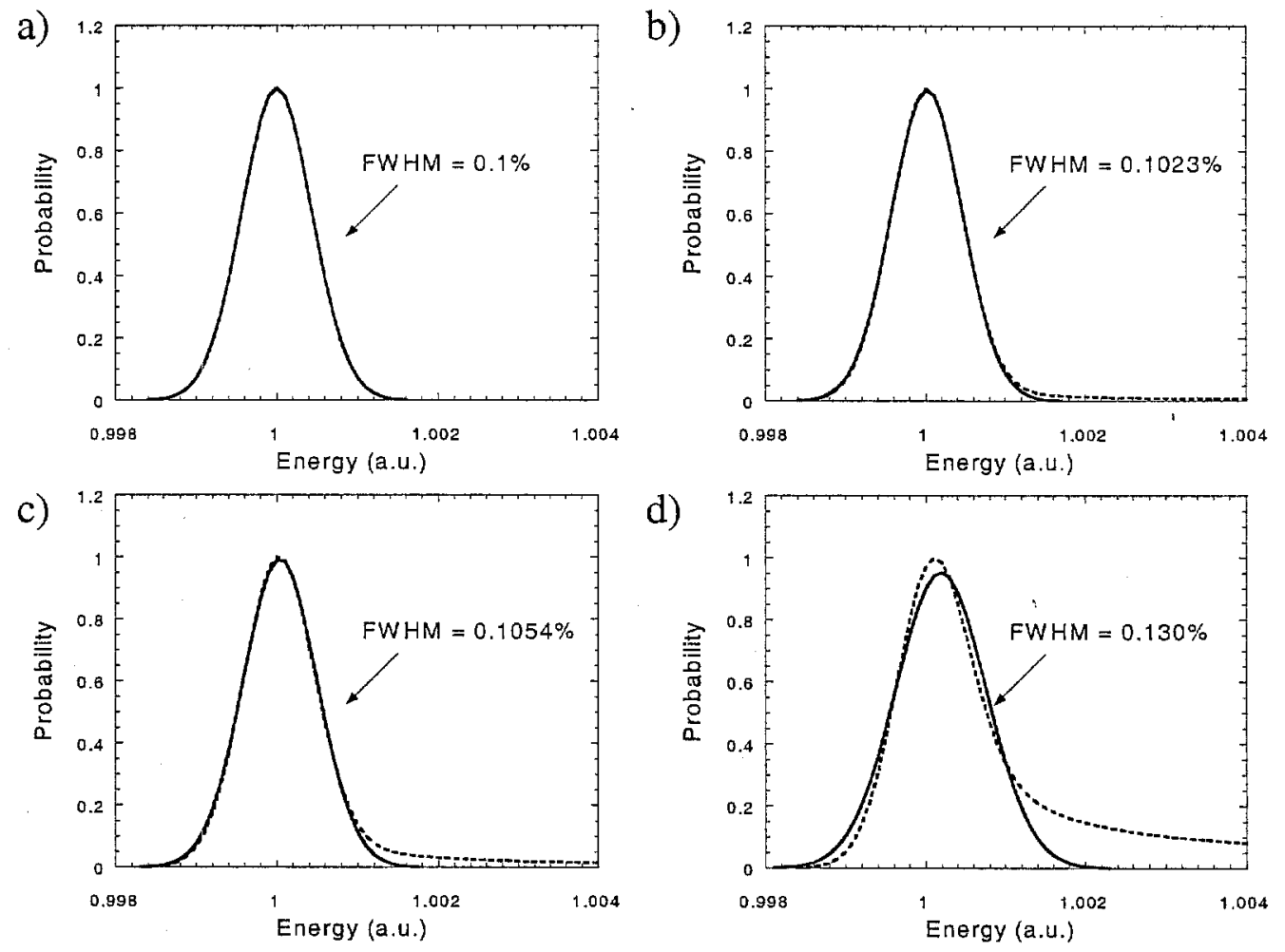

Fig. 2.10 Spectral line broadening due to pile-up events.

a) Spectral peak without pile-up. Energy resolution is $0.1 \%$ for this example. b) Average count rate $\mathrm{n}=$ $1 / 45 \tau$. Energy resolution slightly broadened to $0.1023 \%$. c) Average count rate $n=1 / 20 \tau$ Energy resolution is $0.1054 \%$. d) Average count rate $1 / 5 \tau$. Energy resolution significantly degraded to $0.130 \%$.

The apparent amplitude of the pile-up events will have a lower bound determined by the length of the time window. For example, if the window length is three decay time constants, then the lower bound of the pile-up amplitude is $E_{\min }=\left(1+e^{-3}\right) E_{0}=1.05 E_{0}$, which is $5 \%$ above the monoenergetic line. If the resolution of the detector is better than 5\% FWHM, then the pile-up events will not overlap with the peak and cause broadening. The pile-up energy distribution can be expressed as 
Eq. 2.19

$$
P_{\text {pile-up }}(E)=\delta\left(E, E_{0}\right) P(0)+\frac{n \tau}{E_{0}}\left(\frac{E-E_{0}}{E_{0}}\right)^{n \tau-1} \text { for }\left(1+e^{-t / \tau}\right) E_{0}<E<2 E_{0}
$$

where $\delta\left(E, E_{0}\right)$ is the Dirac delta function, $P(0)$ is the probability of no pile-ups (Eq. 2.14) during the window time $\tau$ with average count rate $n$. The first term is a delta function at $E=E_{0}$, the second term is continuous over the range $\left(1+e^{-\mathrm{t} / \tau}\right) E_{0}<E<2 E_{0}$. This distribution is normalized.

The analysis provided in this section assumes only the pile-up of one pulse on to an existing decay tail. This analysis is not valid to extremely high count rates where two or more pile-ups are likely. This analysis also assumes that the gain of the detector is independent of the baseline. This is not true for some detectors. Line broadening due to baseline gain shifts as a function of count rate is discussed in Section 3.6 on page 59 .

\subsection{Summary of spectral features}

Given the complexity of all the processes involved in the detection process, it is not surprising that many spectral features arise from the incidence of monoenergetic photons. Photoelectric absorption, Compton scattering, and pair production (Section 2.2) all produce secondary photons. If any of these photons escape from the absorber volume, then the energy measured by the detector will be less than the incident energy. Fig. 2.11 shows some possible spectral features from the three absorption processes discussed here. 


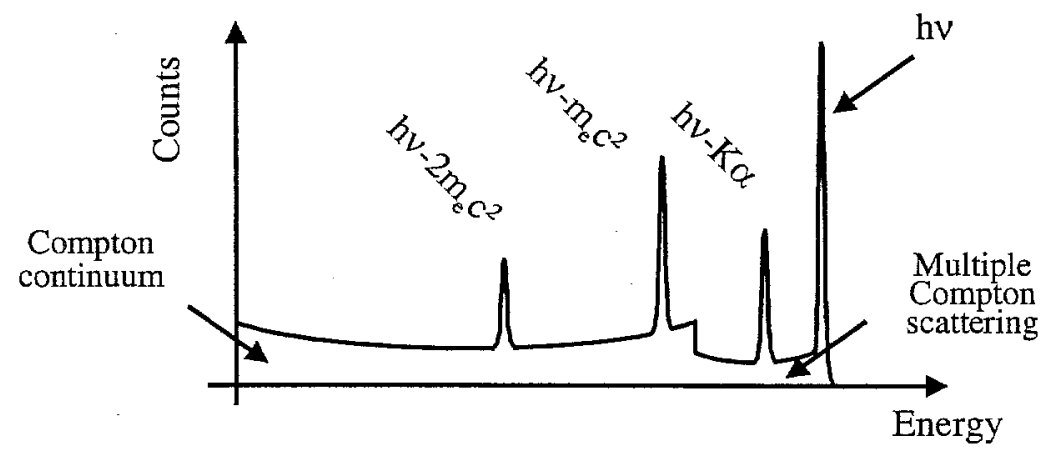

Fig. 2.11 Illustration of spectral features.

The full incident energy is denoted by hv. Photoelectric escape line has energy hv-K $\alpha$, where $\mathrm{K} \alpha$ is the binding energy of the photoelectron. Pair production escape lines have energies $h v-m_{e} c^{2}$ and $h v-2 m_{e} c^{2}$ for the escape of one and both annihilation photons, respectively.

Consider the measured spectrum from a source of monoenergetic photons with energy $h v$. If the secondary photon from photoelectric absorption escaped, then a spectrum line appears corresponding to the energy $h \nu-K \alpha$, where $K \alpha$ is the binding energy of the photoelectron. Similarly, if one or both annihilation gamma-rays from pair production escaped, the spectrum will show a line at $h v-m_{e} c^{2}$ and $h v-2 m_{e} c^{2}$, respectively. Compton scattering will produce a continuum of events from zero energy up to the edge of the Compton gap. These features result from the interaction of the incident photon with the absorbing material via the three processes. Secondary photons may not necessarily escape, but may interact with the absorber again using the three fundamental processes. This results in a more complex set of escape lines. The Compton gap may become indistinct, due to multiple Compton scattering events. To further complicate matters, backscatter and fluorescence from neighboring material will also contribute to the measured spectrum. In the end, the measured spectrum will exhibit many features from the many possible interactions with the detector and its surroundings. 


\section{References}

[1] G.F. Knoll, Radiation Detection and Measurement, John Wiley \& Sons, New York (1979).

[2] R.D. Evans, The Atomic Nucleus, Krieger, New York (1982). 
Chapter 3

\section{MiCROCALORIMETERS}

\section{AND THE TES}

\subsection{Microcalorimeters}

Microcalorimeters generally have three distinct components: the absorber, the thermometer, and the thermal link to a cold bath. Photons are absorbed in the absorber, creating excitations that eventually turn into heat. The change in temperature due to the absorption of a photon depends on the heat capacity of the detector,

Eq. 3.1

$\Delta T=E / C$,

where $E$ is the energy of the photon and $C$ is the heat capacity of the detector. A small heat capacity means a larger change in temperature. Therefore, it is preferable to use materials with small heat capacities. The subsequent increase in temperature is measured by a sensitive thermometer. For this dissertation, the thermometer is a superconducting transition-edge sensor (TES). The TES is a superconducting thin film with a sharp 
transition from superconducting state to the normal state at the critical temperature $T_{c}$. When the TES is biased on the steep transition, it is sensitive to very small changes in temperature, which cause large changes in electrical resistance. The change in resistance can be measured with the appropriate bias. The TES is described in detail in Section 3.3 on page 46. After the absorption of a photon, the excess heat is drained into the cold bath via the thermal link, thus restoring equilibrium. For a good measurement, it is important that the thermal coupling between the absorber and the TES be much stronger than the thermal coupling between the TES and the cold bath. This ensures that very little heat "leaks" out of the detector prior to complete thermalization and measurement. The expected signal for absorption of a photon is a fast rise time (corresponding to the thermalization time of the detector), and a slower exponential decay time (corresponding to the slow dissipation of heat through the weak thermal link to the cold bath). The height of the pulse is ideally proportional to the change in temperature of the detector.

\subsection{Introduction to superconductivity}

\subsubsection{Phenomenon: perfect conductivity and perfect diamagnetism}

The phenomenon of superconductivity was discovered in 1911 by Kammerlingh Onnes [1]. With the newly discovered helium liquefying process, he was able to reach record low temperatures. He observed that mercury, as well as many other metals and alloys, had no electrical resistance below a critical temperature $T_{c}$, which is material dependent. Fig. 3.1 shows the classic plot made by Kammerlingh Onnes showing the transition of $\mathrm{Hg}$ to the superconducting state. 


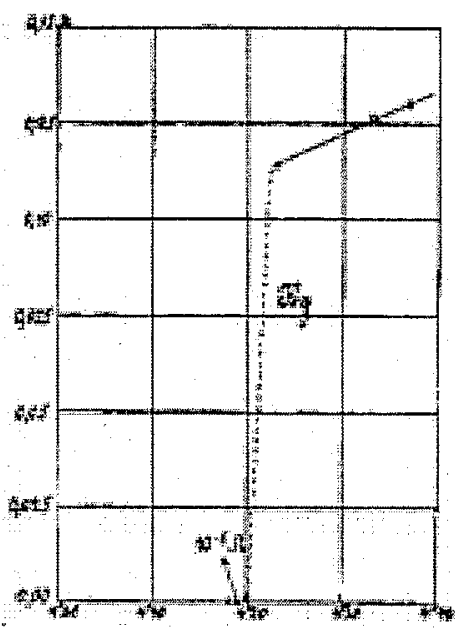

Fig. 3.1 Transition of $\mathrm{Hg}$ to superconducting state observed by Kammerlingh Onnes.

The second phenomenon associated with superconductivity was discovered by Meissner and Ochsenfeld in 1933 [2]. They discovered that magnetic fields were expelled from the bulk volume when materials transit into the superconducting state. This is commonly referred to as the Meissner effect. This result cannot be derived by assuming that a superconductor simply has zero resistivity. From Ohm's law, we know that $\mathbf{E}=\rho \mathbf{J}$, where $\mathbf{E}$ is the electric field, $\rho$ is the resistivity, and $\mathbf{J}$ is the current density. Electrical currents can pass through a superconductor, so if $\rho$ is zero, then the electric field $\mathbf{E}$ must also be zero. From Maxwell's equation $\nabla \times \mathbf{E}=(1 / c)(\partial \mathbf{B} / \partial t)$, zero electric field requires the magnetic field to be constant in time. Thus, magnetic field should be "frozen" in a superconductor, not expelled. This contradicts the Meissner effect, suggesting that perfect diamagnetism is a fundamental property of the superconducting state. Superconductivity can be destroyed by application of strong magnetic field. The superconducting state has a Helmholtz free energy less than the normal state. However, 
the additional energy required to expel a magnetic field can be large enough that it is more favorable for the material to be in the normal state. The critical field is

Eq. 3.2

$$
\frac{H_{c}^{2}(T)}{8 \pi}=f_{n}(T)-f_{s}(T)
$$

where $f_{n}$ and $f_{s}$ are the Helmholtz free energies of the normal and superconducting states. Empirically, the critical field is found to vary with temperature according to

$$
H_{c}(T) \approx H_{c}(0)\left[1-\left(T / T_{c}\right)^{2}\right]
$$

where $H_{c}(0)$ is the critical field at zero temperature. The existence of the critical field implies that there is also a critical current $I_{c}$, which is the necessary current flowing through the superconductor to create the critical field, thereby suppressing superconductivity.

In 1935, the London brothers proposed two equations to describe the microscopic electric and magnetic fields in a superconductor [3]

Eq. 3.4

$$
\mathbf{E}=\frac{\partial}{\partial t}\left(\Lambda \mathbf{J}_{s}\right)
$$

Eq. 3.5

$$
\mathbf{H}=-c \nabla \times\left(\Lambda \mathbf{J}_{\mathbf{s}}\right),
$$


where $\Lambda=m_{e} / n_{s} e^{2}$, and $n_{s}$ is the number of superconducting electrons. These are known as the first (Eq. 3.4) and second (Eq. 3.5) London equations. The first London equation describes perfect conductivity. An electric field accelerates the superconducting electrons, rather than simply maintain the velocity of electrons against resistance as described by Ohm's law in the normal state. The second London equation can be combined with Maxwell's equation $\nabla \times \mathbf{H}=(4 \pi / c) \mathbf{J}$ to get

Eq. 3.6

$$
\nabla^{2} \mathbf{H}=\mathbf{H} / \lambda^{2}
$$

where $\lambda^{2}=(c / 4 \pi) \Lambda$. This describes the exponential attenuation of magnetic fields with a penetration depth $\lambda$, which is the Meissner effect.

\subsubsection{Origin of superconductivity: Coopers pairs and BCS energy gap}

The superconducting transition temperature is dependent not only on the element, but also on the particular isotope. For isotopes of the same element, $T_{c}$ varies approximately with $M^{a}$, where $\mathrm{M}$ is the atomic mass and $a$ is roughly 0.5 for some elements. The only reason for $T_{c}$ to vary with the number of neutrons in the nucleus is that superconductivity must be based on electron-lattice interactions. In 1956, Cooper presented his derivation that the Fermi sea is unstable against the formation of bound electron pairs, regardless of how weak the attractive potential [4]. Such a potential does exists when one considers deformation of the lattice by electrons. An electron will attract a positive nucleus and polarize the medium. Another electron attracted to the same nucleus will respond to its motion. Therefore, the second electron has an indirect attraction to the first electron. If 
this attraction is greater than the screened Coulomb repulsion between the two electrons, then it results in a net attraction. At high temperature few electrons are bound in Cooper pairs due to thermal motion. As temperature decreases, more and more electrons condense into Cooper pairs, altering the Fermi sea. Equilibrium is reached when the Fermi state is so far altered that the binding energy for an additional pair of electrons is zero. The formalism to describe this state was given by Bardeen, Cooper, and Schrieffer (BCS) in 1956 [5]. Note that the concept of Cooper pairs should not be taken too literally. Bound pairs are not in close proximity with each other. In fact, they are much further apart than the average interparticle distance [6]. The average distance between electrons in a Cooper pair is known as the coherence length. The participating electrons are also exchanged frequently. The notion of the Cooper pair is simply that the electrons are no longer behaving as fermions, but as bosons. BCS theory describes the superconducting state as having an energy gap between the ground state and excited states. This is analogous to the band gap of semiconductors. One of the prominent results produced by BCS theory is the prediction of the transition temperature, [5]

Eq. 3.7

$$
T_{c}=1.14 \theta_{D} \exp \left(-\frac{1}{U D\left(\varepsilon_{F}\right)}\right)
$$

where $\theta_{\mathrm{D}}$ is the Debye temperature, $U$ is the attractive electron-lattice interaction, and $D\left(\varepsilon_{\mathrm{F}}\right)$ is the electron density. The value of $U$ can be estimated from electrical resistivity at room temperature, where it is dominated by electron-phonon interactions. It is interesting to note that higher resistivity corresponds to larger values of $U$ and higher values of $T_{c}$. In other words, the more resistive a material is at room temperature, the 
more likely it will superconduct at low temperature. Eq. 3.7 is more commonly expressed in terms of the energy gap

Eq. 3.8

$$
\Delta(0)=1.764 k T_{c},
$$

where $k$ is Boltzmann's constant, and $\Delta(0)$ is the gap at $T=0$. For temperatures near $T_{c}$, the energy gap has a temperature dependence approximated by

Eq. 3.9

$$
\frac{\Delta(T)}{\Delta(0)} \approx 1.74 \sqrt{1-\frac{T}{T_{c}}}
$$

which has a nearly vertical tangent at $T=T_{c}$. The gap emerges rapidly just below $T_{c}$. The number of normal electrons decrease exponentially at temperatures below $T_{c}$,

Eq. $3 \cdot 10$

$$
n_{n} \sim e^{-\Delta / k T}
$$

\subsubsection{Thermal properties: heat capacity}

One consequence of the energy gap is a discontinuity of the heat capacity across the superconducting transition. At temperatures above $T_{c}$, the gap is zero, and the heat capacity of the material is dominated by the normal metal electrons [7]

Eq. 3.11

$$
C_{e}=\frac{1}{2} \pi^{2} N k \frac{T}{T_{F}}=\gamma V T,
$$


where $N$ is the number of electrons, $T_{F}$ is the Fermi temperature, and $V$ is the volume of the material. Below $T_{c}$ the heat capacity has an exponential dependence

Eq. 3.12

$$
C_{s}=\gamma V T_{c} a e^{\frac{b T_{c}}{T}}
$$

where $a$ and $b$ are numerical constants. At $T_{c}$, where the energy gap suddenly appears, the heat capacity is discontinuous. The magnitude of the discontinuity is

Eq. 3.13

$$
C_{s}\left(T_{c}\right)=2.43 C_{e}\left(T_{c}\right)
$$

At low temperatures, where there are very few normal electrons, the heat capacity is dominated by the phonon heat capacity, given by [7]

Eq. 3.14

$$
C_{p}=\frac{12 \pi^{4}}{5} N k\left(\frac{T}{\theta_{D}}\right)^{3}=\alpha V T^{3},
$$

where $N$ is the number of phonon modes and $\theta_{D}$ is the Debye temperature..

\subsubsection{Quantum state: Ginzburg-Landau order parameter}

Whereas BCS is a microscopic theory of superconductivity, the Ginzburg-Landau (GL) theory is a macroscopic one. GL takes into account spatial inhomogeneity. The basis of the GL theory is the introduction of a spatially-dependent "order parameter" denoted by 
$\psi(\mathbf{x})$. The magnitude of the order parameter describes the local density of superconducting electrons $n_{s}(\mathbf{x})$,

Eq. 3.15

$$
|\psi(\mathbf{x})|^{2}=n_{s}(\mathbf{x})
$$

The order parameter is treated as a wavefunction of the superconducting state. 'The GL theory is a generalization of the London theory, with $n_{s}$ varying in space rather than constant. GL theory also introduces us to the flux quantum. Consider a superconducting ring with the GL wavefunction written as

Eq. 3.16

$$
\psi(\mathbf{x})=\sqrt{n_{s}} e^{i \theta(\mathbf{x})}
$$

where $\theta(x)$ is the phase. In order for the wavefunction to be single-valued, the phase difference around the ring must be integer multiples of $2 \pi$. The magnetic flux through the ring must take on discrete values with the smallest value being the flux quantum, given by

Eq. 3.17

$$
\Phi_{0}=h c / 2 e \approx 2.0678 \times 10^{-7} \text { Gauss } \mathrm{cm}^{2}
$$

In 1962, Josephson derived two predictions about superconducting tunnel junctions $[8,9]$. A superconducting tunnel junction consists of two superconductors separated by a thin insulator such that superconducting electron pairs may tunnel from one superconductor to the other. Let the order parameters of the two superconductors be $\psi_{1}$ and $\psi_{2}$, where $\psi$ 
has the form given by Eq. 3.16. By applying the time-dependent Schrödinger equations for both superconductors in the absence of a potential and solving for the current flow, we get

Eq. 3.18

$$
J=J_{0} \sin \delta
$$

where $\delta=\theta_{2}-\theta_{1}$ is the phase difference across junction and $J_{0}$ is dependent on the tunneling probability. This is known as the dc Josephson effect. It predicts that, even without a voltage difference, a supercurrent can flow across the junction. The magnitude of the current depends on the phase difference across the junction. This effect is exploited by superconducting quantum interference devices (SQUIDs) discussed in detail in Section 6.2.1 on page 116. If a voltage is applied across the junction, the supercurrent is

Eq. 3.19

$$
J=J_{0} \sin \left(\delta(0)-\frac{2 e V t}{\hbar}\right)
$$

This is known as the ac Josephson effect. The supercurrent will oscillate with a frequency $\omega=2 \mathrm{eV} / \hbar$. This relationship is commonly used to set voltage standards.

\subsubsection{Basis of the TES: Phase transition}

The onset of electrical resistance as temperature is increased to approach $T_{c}$ is described in terms of phase slips [10]. Consider a one-dimensional superconducting wire. For 
perfect conductivity, there must be no voltage drop across the length of the wire. From the second Josephson relation, this implies that the relative phase difference $\delta$ between the two ends must be constant. However, at temperatures a few millikelvin below $T_{c}$, thermal fluctuations may momentarily break up coherence of the wavefunction and allow the phase to slip by a multiple of $2 \pi$ before regaining coherence. The rate at which these phase slips occur correspond to a voltage drop across the wire

Eq. 3.20

$$
\frac{d \delta}{d t}=\frac{2 e V}{\hbar}
$$

For a constant current, as the temperature approaches $T_{c}$, the probability of phase slips increase as well as the voltage and resistance. This is the mechanism for the emergence of resistance in the phase transition. The mechanism for normal state resistance at low temperatures is impurity scattering of electrons. At high temperatures, the mechanism for resistance is electron-phonon scattering. Phase slips from thermal fluctuations have an associated voltage noise [11]

Eq. 3.21

$$
\left|V_{\text {slips }}\right|=\sqrt{\frac{\Phi_{0} \delta}{\pi} V}
$$

\subsection{Operating principles of the TES}

In principle, the superconducting transition of the TES is a function of both temperature and current. Extensive theory has been developed to address the dependencies on current [12]. For the detectors described in this dissertation, we did not observe significant 
variation of the transition with current (Section 7.4, page 134). Thus, we can neglect current effects on the superconducting transition.

We have designed our TES with an operating temperature of approximately $100 \mathrm{mK}$ and a heat capacity approximately $100 \mathrm{keV} / \mathrm{mK}$. The temperature rise from absorption of a $60 \mathrm{keV}$ gamma ray is less than $1 \mathrm{mK}$. For a microcalorimeter dominated by phonon heat capacity, a $1 \%$ change in temperature corresponds to a $3 \%$ change in heat capacity. Therefore, we can assume that heat capacity to be constant in the range between the operating temperature and the small temperature rise from absorption of a gamma ray. Similarly, we assume that thermal couplings are constant at the operating temperature.

The simplest description of a TES is a temperature-dependent resistor. The operating bias point is at resistance $R_{0}$ and temperature $T_{0}$. The sensitivity of the resistor to temperature changes is related to $d R / d T$. For a superconducting film biased on the transition, $d R / d T$ is positive. We use the dimensionless parameter

Eq. 3.22

$$
\alpha=\frac{d(\log R)}{d(\log T)}=\frac{T_{0}}{R_{0}} \frac{d R}{d T}
$$

which depends on the bias point and the slope of the transition. We can derive a simple expression for the response of a TES from Ohm's law $I=V I R$, where $I, V$, and $R$ are the current, voltage, and resistance of the TES, respectively. When we apply a voltage bias and assume that $R$ varies only with $T$ and not with $l$, the change in current due to a small change in temperature is given by the Taylor expansion of Ohm's law about $T_{0}+\Delta T$, 
Eq. 3.23

$$
I=I_{0}-\frac{I_{0}}{R_{0}} \frac{d R}{d T} \Delta T+\cdots
$$

where we recognize the first term on the right side as the steady-state current. The second term is the first-order signal response to a small change in temperature, ,

Eq. 3.24

$$
I_{\text {Signal }}=-\frac{I_{0}}{R_{0}} \frac{d R}{d T} \Delta T=-I_{0} \alpha \frac{\Delta T}{T_{0}}
$$

For a microcalorimeter, the steady state temperature is $T_{0}$ and the change in temperature is given by Eq. 3.1. From Eq. 3.24, we see that for a voltage biased TES with positive $\alpha$, we expect the signal to be a negative current pulse. The amplitude of the pulse is proportional to the steady state current, the steepness of the transition, and the change in temperature.

\subsection{Analytical model}

In a TES microcalorimeter, the thermal properties and electrical properties are coupled, creating electrothermal feedback. A simple microcalorimeter with a TES weakly coupled to the cold bath is shown in Fig. 3.2. 


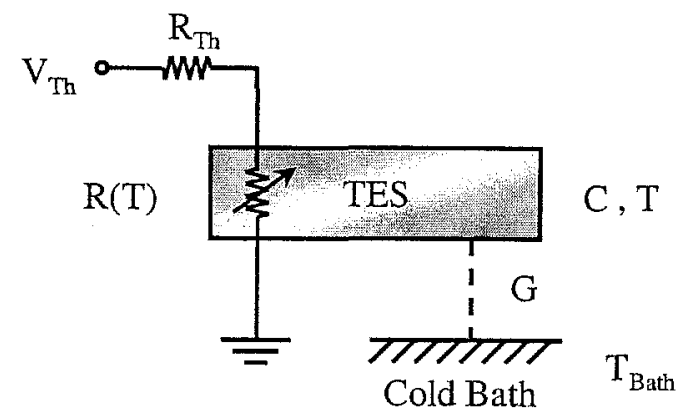

Fig. 3.2 Schematic diagram of a TES with operating bias coupled to cold bath.

The heat capacity of the TES is $C$, temperature of the TES is $T$, temperature of the bath is $T_{B a t h}$, thermal conductivity between TES and cold bath is $G$, resistance of the TES is $R(T)$, and operating bias is provided by $V_{T h}$ and $R_{T h}$. According to Thévenin's theorem, we can simplify the actual bias circuit with its Thévenin equivalent using $V_{T h}$ and $R_{T h}$ in series with the TES as we have done in Fig. 3.2 [13]. In the absence of any bias, the temperatures of the TES and the bath are equal. When a bias is applied, the temperature of the TES will be greater than the bath due to Ohmic heating. We can write a first-order differential equation expressing the power flow in this TES [14]

Eq. 3.25

$$
C \frac{d T}{d t}=P_{B i a s}-K \cdot\left(T^{N}-T_{B a t h}^{N}\right)
$$

where $P_{\text {Bias }}=I V$ is the applied bias power, $I$ and $V$ are the current and voltage of the TES, respectively, $K$ is the thermal conductance to the cold bath, and the factor $N$ depends on the cooling mechanism. The thermal conductivity is given by 
Eq. 3.26

$$
G=\frac{d P}{d T}=N K T^{N-1}
$$

The voltage drop across the TES is given by

Eq. 3.27

$$
V=V_{T h}\left(\frac{R(T)}{R_{T h}+R(T)}\right)
$$

Combining this expression with Ohm's law and substituting into Eq. 3.25, we have

Eq. 3.28

$$
C \frac{d T}{d t}=\frac{V_{T h}^{2} R(T)}{\left(R_{T h}+R(T)\right)^{2}}-K \cdot\left(T^{N}-T_{B a t h}^{N}\right) .
$$

Linearizing this differential equation about $T=T_{0}$ with perturbation $\Delta T$, we find

Eq. 3.29

$$
C \frac{d}{d t} \Delta T=-\left[1+\frac{\alpha}{N}\left(1-\frac{T_{\text {Bath }}^{N}}{T_{0}^{N}}\right)\left(1-\frac{2 R_{0}}{R_{\text {Th }}+R_{0}}\right)\right] G \Delta T,
$$

where $T_{0}$ and $R_{0}$ is the steady state temperature and resistance of the TES. The differential equation has an exponential solution with an effective time constant

Eq. 3.30

$$
\tau=\frac{\tau_{0}}{1+\frac{\alpha}{N}\left(1-\frac{T_{\text {Bath }}^{N}}{T_{0}^{N}}\right)\left(1-\frac{2 R_{0}}{R_{T h}+R_{0}}\right)},
$$


where $\tau_{0}=C / G$ is the "natural" time constant of the system in the absence of any bias. When we let $R_{T h} \rightarrow \infty$, we have a constant currant bias on the TES. The time constant is

Eq. 3.31

$$
\left.\tau\right|_{R_{T h} \rightarrow \infty}=\frac{\tau_{0}}{1-\frac{\alpha}{N}\left(1-\frac{T_{B a t h}^{N}}{T_{0}^{N}}\right)}>\tau_{0}, \text { for positive } \alpha
$$

The time constant of a TES under a constant current bias is greater than the natural time constant. We refer to this as positive electrothermal feedback. A constant current bias is stable only for small $\alpha$ or small bias power. For large $\alpha$ or large bias power, the TES will usually saturate in the normal state and not recover its bias point on the transition.

When we let $R_{T h} \rightarrow 0$, we have a constant voltage bias. The time constant is

Eq. 3.32

$$
\left.\tau\right|_{R_{\text {Th }} \rightarrow 0}=\frac{\tau_{0}}{1+\frac{\alpha}{N}\left(1-\frac{T_{B u t h}^{N}}{T_{0}^{N}}\right)}<\tau_{0}, \text { for positive } \alpha
$$

The recovery time of a constant voltage biased TES is shorter than the natural time constant. We refer to this as negative electrothermal feedback. With a constant voltage bias, the TES is stable for all positive $\alpha$. The "stiffness" of the voltage bias depends on the values of $R_{0}$ and $R_{T h}$. A near-constant voltage bias can be achieved for $R_{T h} \leq 0.1 R_{0}$.

The exponential response of the TES can be incorporated into Eq. 3.24, giving 
Eq. 3.33

$$
I_{\text {Signal }}(t)=-\frac{I_{0} \alpha E}{C T_{0}} \exp \left(-\frac{t}{\tau}\right)
$$

This assumes that the rise time of the pulse is instantaneous. In most microcalorimeters, the rise time is much faster than the decay time, so Eq. 3.33 is a valid approximation. The current power spectral density for the signal is given by its Fourier transform

Eq. 3.34

$$
I^{2}(\omega)=\left(\frac{I_{0} \alpha E}{C T_{0}}\right)^{2} \frac{\tau^{2}}{1+\omega^{2} \tau^{2}}
$$

The spectral power density is flat at low frequency, and has a roll-off knee at $1 / \tau$.

\subsection{Intrinsic noise sources}

The microcalorimeter has two intrinsic noise sources: phonon noise (thermal fluctuations), and Johnson noise (voltage fluctuations). There are external noise sources as well, which will be considered later. A good treatment of microcalorimeter noise in general is given by Mosely, Mather, and McCammon [15] and for the case of a TES in the constant voltage bias limit is given by Irwin [16][17]. A complete generalization of microcalorimeter noise with current dependence is described by Lindeman [12]. In this section, we derive the expressions for noise in a microcalorimeter in a similar fashion as Irwin [16][17], but without using approximations that are not valid for our detector. 


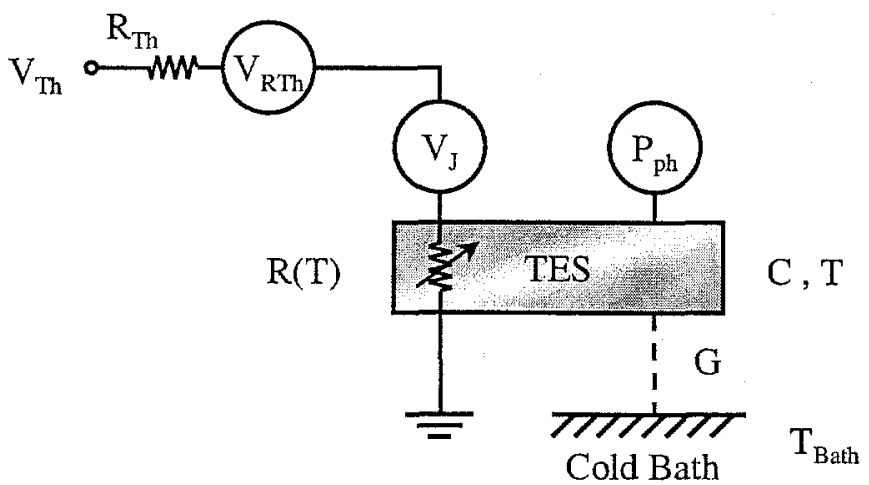

Fig. 3.3 Simple TES with noise contributions.

We begin by adding noise contributions to the illustration of the TES and bias as shown in Fig. 3.3.Phonon noise affects the response of the TES by producing fluctuations in the temperature of the TES. The Johnson noise of the TES gives rise to fluctuations of the voltage across the TES. TES Johnson noise affects the bias power dissipated in the TES as well as the current passing through the TES. The Thévenin bias resistor also has intrinsic Johnson noise. However, bias Johnson noise only affects the current, not the bias power of the TES. The differential equation describing the TES with noise contributions is

Eq. 3.35

$$
C \frac{d T}{d t}=\frac{V_{T h}^{2} R(T)}{\left(R_{T h}+R(T)\right)^{2}}-K \cdot\left(T^{N}-T_{B a t h}^{N}\right)+P_{J}(t)+P_{P h}(t),
$$

where $P_{J}(t)=I_{0} V_{J}(t)=V_{T h} V_{J}(t) /\left(R_{T h}+R_{0}\right)$ is the TES Johnson noise term and $P_{P h}(t)$ is the phonon noise term. Taking the Fourier transform of the differential equation, we find 
Eq. $3.36 i \omega C T(\omega)=\frac{V_{T h}^{2}\left(R_{T h}-R_{0}\right)}{\left(R_{T h}+R_{0}\right)^{3}} \frac{d R}{d T} T(\omega)-G T(\omega)+\frac{V_{T h} V_{J}(\omega)}{R_{T h}+R_{0}}+P_{P h}(\omega)$,

where $T(\omega)$ is the temperature in the frequency domain. Solving for $T(\omega)$ and using Eq. 3.24 to determine the noise current due to temperature fluctuations, we find

Eq. 3.37

$$
I_{N}(\omega)=\frac{V_{J}}{R_{T h}+R_{0}}+\frac{V_{R T h}}{R_{T h}+R_{0}}-\frac{V_{T h} \alpha}{\left(R_{T h}+R_{0}\right) T_{0}} T(\omega)
$$

where

$$
T(\omega)=\frac{\frac{V_{T h} T_{0}}{\left(R_{T h}+R_{0}\right)} V_{J}(\omega)+T_{0} P_{P h}(\omega)}{G T_{0}-V_{T h}^{2} \alpha \frac{\left(R_{T h}-R_{0}\right)}{\left(R_{T h}+R_{0}\right)^{3}}+i \omega C T_{0}}
$$

The first term is current noise due to TES Johnson noise. The second term is current noise due to bias Johnson noise. The third term is the current noise due to temperature fluctuations, which depends on the phonon noise and also the TES Johnson noise. Collecting noise terms, we get the current power spectral densities

Eq. 3.38

$$
I_{J}^{2}(\omega)=V_{J}^{2}\left|\frac{1}{R_{T h}+R_{0}}-\frac{V_{T h}{ }^{2} \alpha /\left(R_{T h}+R_{0}\right)^{2}}{G T_{0}-V_{T h}{ }^{2} \alpha R_{0} \frac{\left(R_{T h}-R_{0}\right)}{\left(R_{T h}+R_{0}\right)^{3}}+i \omega C T_{0}}\right|^{2},
$$

Eq. 3.39

$$
I_{P h}^{2}(\omega)=P_{P h}^{2}\left|\frac{V_{T h} \alpha /\left(R_{T h}+R_{0}\right)}{G T_{0}-V_{T h}^{2} \alpha R_{0} \frac{\left(R_{T h}-R_{0}\right)}{\left(R_{T h}+R_{0}\right)^{3}}+i \omega C T_{0}}\right|^{2}
$$

and 
Eq. 3.40

$$
I_{R t h}^{2}(\omega)=\left(\frac{V_{R T h}}{R_{T h}+R_{0}}\right)^{2}
$$

The first equation is the Johnson noise of the TES, where $V_{J}^{2}=4 k T_{0} R_{0}$. Because of the electrothermal coupling, there is some correlation between the current fluctuations due to voltage fluctuations and due to bias power fluctuations. Eq. 3.38 shows the result of this correlation: Johnson noise is suppressed at frequencies below $1 / \tau$, but resumes the white noise value at higher frequencies. The second equation is the phonon noise of the TES, where $P_{P h}{ }^{2}=2 k\left(T_{0}{ }^{2}+T_{B a t h}{ }^{2}\right) G$. For voltage bias $\left(R_{T h} \rightarrow 0\right)$ and strong electrothermal feedback $\left(T_{0} \gg T_{B a t h}\right)$, the phonon noise has the same shape as the power spectrum of the signal, flat at low frequency and rolls off at the frequency $1 / \tau$. The third equation is the Johnson noise of the bias resistor, where $V_{R T h}^{2}=4 k T_{R T h} R_{T h}$, where $T_{R T h}$ is the temperature of the bias resistor.

The expressions given above are only valid for negligible inductance and capacitance in the bias circuit. In the real bias circuit, however, there is a small series inductance $L$ for coupling to a SQUID current amplifier (Section 6.2.1, page 116). We may incorporate inductive effects by letting $R_{T h} \rightarrow R_{T h}+i \omega L$ in the Fourier transform of the differential equation,

Eq. 3.41 $I_{N}(\omega)=\frac{V_{J}}{R_{T h}+i \omega L+R_{0}}+\frac{V_{R T h}}{R_{T h}+i \omega L+R_{0}}-\left.\frac{V_{T h} \alpha}{\left(R_{T h}+i \omega L+R_{0}\right) T_{0}} T(\omega)\right|_{R_{R h} \rightarrow R_{T h}+i \omega L}$. 
The individual noise terms are

Eq. 3.42

$$
I_{J}^{2}(\omega)=V_{J}^{2}\left|\frac{1}{R_{T h}+i \omega L+R_{0}}-\frac{V_{T h}^{2} \alpha /\left(R_{T h}+i \omega L+R_{0}\right)^{2}}{G T_{0}-V_{T h}^{2} \alpha \frac{\left(R_{T h}+i \omega L-R_{0}\right)}{\left(R_{T h}+i \omega L+R_{0}\right)^{3}}+i \omega C T_{0}}\right|^{2},
$$

Eq. 3.43

$$
I_{P h}^{2}(\omega)=P_{P h}^{2}\left|\frac{V_{T h} \alpha /\left(R_{T h}+i \omega L+R_{0}\right)}{G T_{0}-V_{T h}^{2} \alpha \frac{\left(R_{T h}+i \omega L-R_{0}\right)}{\left(R_{T h}+i \omega L+R_{0}\right)^{3}}+i \omega C T_{0}}\right|^{2}
$$

Eq. 3.44

$$
I_{R T h}^{2}(\omega)=\left|\frac{V_{R T h}}{R_{T h}+i \omega L+R_{0}}\right|^{2},
$$

where $V_{J}$ and $V_{R T h}$ are unchanged, assuming the inductor has no resistance and voltage fluctuations. The TES Johnson noise term is suppressed at frequencies below $1 / \tau$. At higher frequencies between $1 / \tau$ and $\left(R_{T h}+R_{0}\right) / L$, it resumes the white noise value. At very frequencies above $\left(R_{T h}+R_{0}\right) / L$, it rolls off. The phonon noise and signal both have a knee at $1 / \tau$ and another one at $\left(R_{T h}+R_{0}\right) / L$. The bias Johnson noise remains flat until $\left(R_{T h}+R_{0}\right) / L$, where it rolls off. Fig. 3.4 shows a sample power spectral density for the signal and various noise terms based on parameters given in Table 3.1. In most cases, the microcalorimeter will have the $\left(R_{T h}+R_{0}\right) / L$ knee at frequencies much higher than the signal knee such that the inductance $L$ may be neglected. The total noise current amplitude can be found by integrating in quadrature the noise at each frequency up to the $\left(R_{T h}+R_{0}\right) / L$ roll-off. For the noise spectral power density shown in Fig. 3.4, we get $\left|I_{P h}\right|=5.22 \mathrm{nA}$, $\left|I_{J}\right|=9.07 \mathrm{nA},\left|I_{T h}\right|=2.83 \mathrm{nA}$., and a total noise of $10.8 \mathrm{nA}$. For the parameters given in 
Table 3.1 , the signal amplitude is $17.8 \mu \mathrm{A}$. The signal-to-noise ratio without any signal processing is $\sim 1650$, giving us an energy resolution $\Delta E=6.1 \mathrm{eV}$ for $E=10 \mathrm{keV}$.

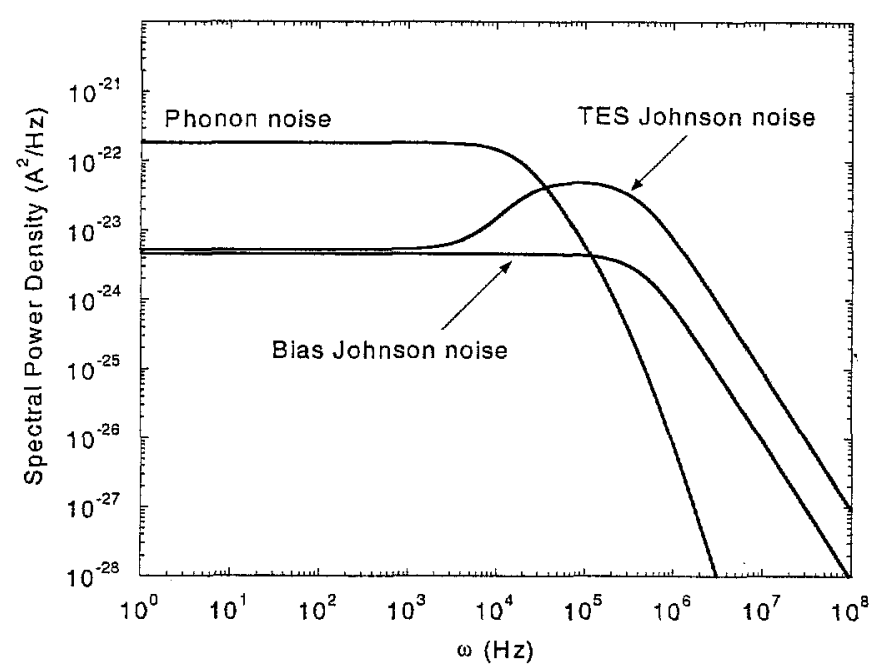

Fig. 3.4 Example of TES noise contributions.

The fundamental limit to the energy resolution of the microcalorimeter can be calculated using by the noise equivalent power. Noise equivalent power (NEP) is defined as the power necessary to generate the current fluctuation. We find NEP by rewriting the differential equation that describes noise in the TES

Eq. 3.45

$$
C \frac{d T}{d t}=\frac{V_{T h}^{2} R(T)}{\left(R_{T h}+R(T)\right)^{2}}-K \cdot\left(T^{N}-T_{\text {Bath }}^{N}\right)+N E P
$$

After Fourier transform and combining with Eq. 3.24 We readily find that 


\begin{tabular}{|c|c|}
\hline Parameter & Value \\
\hline$C$ & $100 \mathrm{keV} / \mathrm{mK}$ \\
\hline$K$ & $250 \mathrm{nW} / \mathrm{K}^{4}$ \\
\hline$N$ & 4 \\
\hline$G$ & $1 \mathrm{nW} / \mathrm{K}$ \\
\hline$L$ & $0.25 \mu \mathrm{H}$ \\
\hline$T_{\text {Bath }}$ & $65 \mathrm{mK}$ \\
\hline$T_{0}$ & $100 \mathrm{mK}$ \\
\hline$R_{0}$ & $100 \mathrm{~m} \Omega$ \\
\hline$R_{\text {Th }}$ & $10 \mathrm{~m} \Omega$ \\
\hline$V_{\text {Th }}$ & $2 \mu \mathrm{V}$ \\
\hline$\alpha$ & 1000 \\
\hline
\end{tabular}

Table 3.1 Example TES parameter values.

Eq. 3.46 $N E P(\omega)=\frac{\left(R_{T h}+R_{0}\right) T_{0}}{V_{T h} \alpha}\left[i \omega C-\frac{V_{T h}^{2} R_{0} \alpha}{T_{0}} \frac{R_{T h}-R_{0}}{\left(R_{T h}+R_{0}\right)^{3}}+G\right] I_{N}(\omega)$,

where $I_{N}(\omega)$ is the appropriate noise current. Fig. 3.5 shows the TES signal and NEP for various noise terms using the same device parameters in Table 3.1. The knee at high frequency is due to the circuit inductance $L$.

The fundamental limit for the energy resolution from the optimal filter is given by [12][18]

Eq. 3.47

$$
\Delta E_{F W H M}=\frac{2.35}{\sqrt{\int_{0}^{\infty} \frac{4 d \omega}{|N E P(\omega)|^{2}}}}
$$




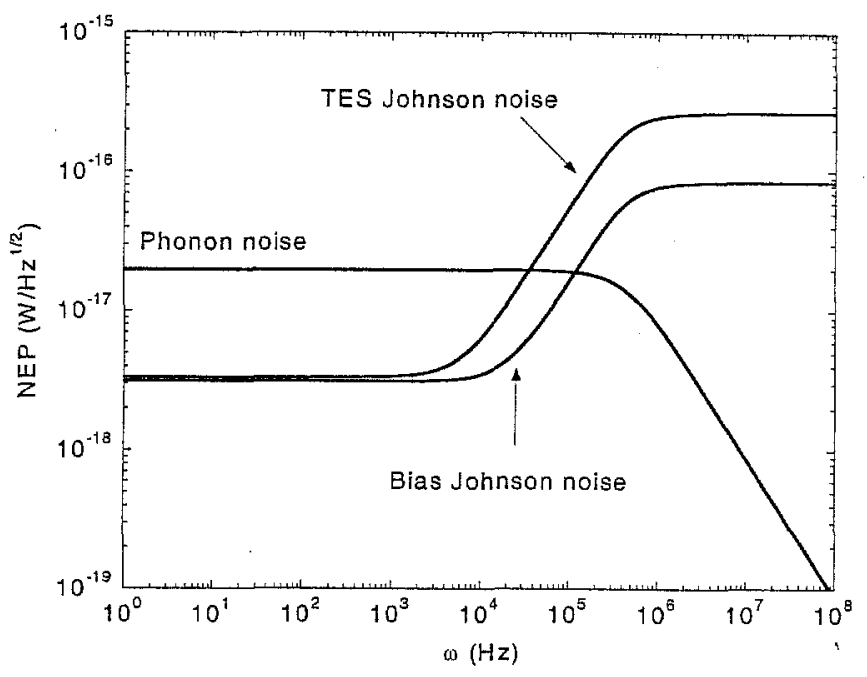

Fig. 3.5 Example of TES noise equivalent power.

For a given device, the fundamental limit for the energy resolution is independent of gamma ray energy. For the parameters used in the example in Table 3.1, the best possible energy resolution is $\sim 2 \mathrm{eV}$. However, for good absorption efficiency at high energy, the absorber must be made larger, thereby increasing heat capacity and phonon noise.

\subsection{Compound microcalorimeters}

The preceding section assumes that the arrival of energy into the TES is instantaneous and has the form of a delta function. This is true if the photons are absorbed directly in the TES film. However, a thin film TES with a typical thickness of $200 \mathrm{~nm}$ has poor absorption efficiency for gamma rays. Thus, we require a thick absorber coupled to the TES. An example of a compound microcalorimeter is shown in Fig. 3.6. In this case, the absorption of the photon energy in the absorber is nearly instantaneous. However, the 
arrival rate of energy into the TES is not instantaneous because of the finite coupling between the absorber and the TES.

If the energy were to arrive gradually over a time of $\tau_{A r r}$, then the TES cannot respond faster than this time scale. In essence, the bandwidth of the microcalorimeter would be limited to $\Delta B \sim 1 / \tau_{A r r}$. For a TES with bias conditions such that the electrothermally shortened time constant is greater than the energy arrival time $\left(\tau>\tau_{A r r}\right)$, then the theoretical noise expressions in Section 3.5 are approximately valid. However, if the TES response time is shorter than the energy arrival time $\left(\tau<\tau_{A r r}\right)$, the theoretical noise expressions may not hold. In this event, both phonon and Johnson noise power terms are bandwidth limited to $1 / \tau_{A r r}[16]$ and dominated by the largest thermal conductance, which is $G_{A b s}$ in this case,

Eq. 3.48

$$
P_{P h} \approx \sqrt{2 k\left(T_{A b s}^{2}+T_{0}^{2}\right) G_{A b s}} \sqrt{\Delta B}=\sqrt{\frac{2 k\left(T_{A b s}^{2}+T_{0}^{2}\right) G_{A b s}}{\tau_{A r r}}},
$$

$$
P_{J} \approx \frac{V_{T h} \sqrt{4 k T_{T E S} R_{0}}}{R_{T h}+R_{0}} \sqrt{\Delta B}=\frac{V_{T h}}{R_{T h}+R_{0}} \sqrt{\frac{4 k T_{T E S} R_{0}}{\tau_{\text {Arr }}}},
$$

A general analytical approach to find the phonon noise spectra for an N-element microcalorimeter is provided by Lindeman, et al [12] and Gildemeister, et al [19]. Applying the derivation to our geometry (Fig. 3.6), we get the temperature power spectral density 
Eq. 3.50

$$
T(\omega)^{2}=\frac{1}{|\operatorname{det}|^{2}}\left(\left(i \omega C_{A b s}+G_{A b s}\right)^{2}\left(S_{A b s}+S_{c o o l}\right)+G_{A b s}^{2} S_{A b s}+2\left(i \omega C_{A b s}+G_{A b s}\right) G_{A b s} S_{C o r r}\right),
$$

with

Eq. 3.51 $\operatorname{det}=\operatorname{det}\left[\begin{array}{cc}\left(i \omega C_{A b s}+G_{A b s}\right) & -G_{A b s} \\ -G_{A b s} & \left(i \omega C_{T E S}+G_{T E S}+G_{A b s}+\frac{P_{B i a s} \alpha}{T_{T E S}}\right)\end{array}\right]$

where $S_{c o o l}=2 k\left(T_{0}^{2}+T_{B a t h}^{2}\right) G_{c o o l}$, and $S_{A b s}=2 k\left(T_{0}^{2}+T_{A b s}{ }^{2}\right) G_{A b s}$, which are the power spectral densities for $G_{c o o l}$ and $G_{A b s}$, respectively. The term $S_{C o r r}$ is a cross-spectral density due to correlation through the same thermal link. For our geometry, $T_{A b s}$ and $T_{T E S}$ are equal at equilibrium, so we assume $S_{\text {Corr }}=0$. The current noise density due to the temperature power spectral density can be found by through Eq. 3.24.

\subsection{Additional noise sources}

There are also external sources of noise that affect the energy resolution of a microcalorimeter. One source of noise is intrinsic to the electronic amplifier. In our case, we use a SQUID amplifier, with typical flat current noise spectral density of 2-10 $\mathrm{pA} / \sqrt{\mathrm{Hz}}$. 


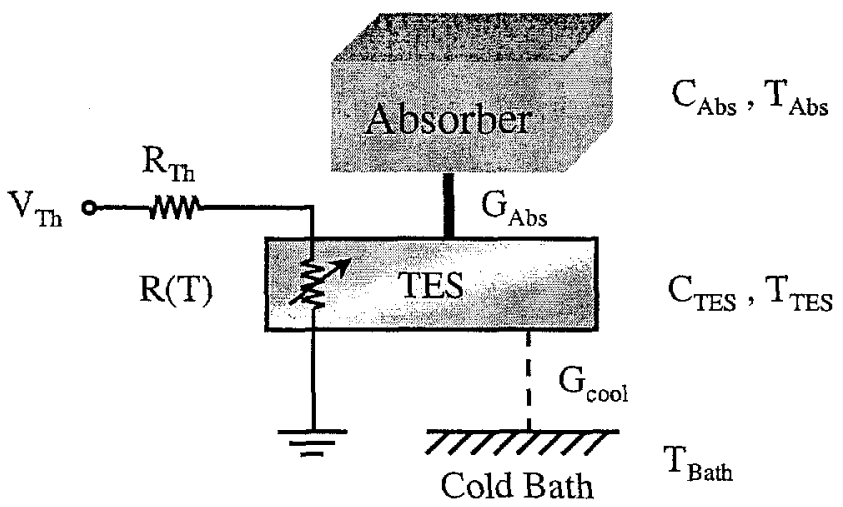

Fig. 3.6 Schematic diagram of a compound microcalorimeter consisting of a TES and an absorber. The TES and absorber have respective heat capacities and temperatures given by $C_{T E S}, C_{A b s}, T_{T E S}$, and $C_{A b s}$.

Fluctuations in the bath temperature also degrade the signal-to-noise ratio. For a TES voltage biased on the transition, electrothermal feedback self-regulates the TES at a near constant $T_{0}$. Fluctuations of the bath do not affect $T_{0}$. The operating temperature is only affected by Johnson noise and phonon noise. Bath fluctuations affects the steady state current $I_{0}$ through the TES. At equilibrium, $P_{\text {Bias }}=I_{0}^{2} R_{0}=K\left(T_{0}{ }^{N}-T_{\text {Bath }}{ }^{N}\right)$. The steadystate current is

Eq. 3.52

$$
I_{0}=\sqrt{\frac{K \cdot\left(T_{0}^{N}-T_{B a t h}^{N}\right)}{R_{0}}} .
$$

The variation in $I_{0}$ due to fluctuations $\delta T_{B a t h}$ is

Eq. 3.53

$$
\delta I_{0}=-\frac{1}{2} \sqrt{\frac{K}{R_{0}\left(T_{0}^{N}-T_{B a t h}^{N}\right)}} N T_{B a t h}^{N-1} \delta T_{B a t h} .
$$


From Eq. 3.24, we see that the gain of the signal is proportional to $I_{0}$. Therefore, the variation of signal amplitude due to bath fluctuations is

Eq. 3.54

$$
\frac{I_{\text {Bath }}}{I_{\text {Signal }}}=\frac{\delta I_{\text {Signal }}}{I_{\text {Signal }}}=\frac{\delta I_{0}}{I_{0}}=\frac{1}{2} \frac{N T_{\text {Bath }}^{N-1} \delta T_{\text {Bath }}}{\left(T_{0}^{N}-T_{\text {Bath }}^{N}\right)},
$$

where $I_{B a t h}$ is the noise current due to bath fluctuations. This noise occurs at frequencies much lower than the frequency domain of the signals, therefore, bath fluctuations may not appear in a noise spectrum of the measured signal. For the example parameters given in Table 3.1 , in order to achieve an energy resolution of $0.1 \%$, the fluctuation in bath temperature $\delta T_{B a t h}$ needs to be smaller than $150 \mu \mathrm{K}$.

Another factor that causes line broadening is baseline gain shift due to pulse pile-up. Unlike the pile-up of simple amplitude addition with constant baseline discussed in Section 2.6.3 (page 28), this is a change in signal gain due to incomplete recovery to thermal equilibrium. According to Eq. 3.24, the signal gain is dependent on $T_{0}$. We can Taylor expand the signal size about $T_{0}+\delta T$ and get

Eq. 3.55

$$
I_{\text {Signal }}=-\frac{I_{0}}{R_{0}} \frac{d R}{d T} \frac{E}{C}+\left[2 \frac{I_{0}}{R_{0}^{2}}\left(\frac{d R}{d T}\right)^{2}-\frac{I_{0}}{R_{0}} \frac{d^{2} R}{d T^{2}}\right] \frac{E}{C} \delta T_{0},
$$

where we assume the superconducting transition to be approximately linear, such that the $d^{2} R / d T^{2}$ term is negligible. We recognize the first term as the signal size at equilibrium, 


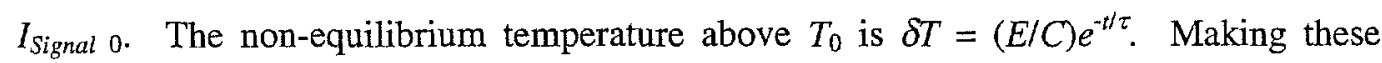
substitutions, we have

Eq. 3.56

$$
I_{\text {Signal }}=I_{\text {Signal } 0}\left(1-2 \frac{\alpha}{T_{0}} \delta T_{0}\right)=I_{\text {Signal } 0}\left(1-2 \frac{I_{\text {Signal0 }}}{I_{0}} e^{-\frac{t}{\tau}}\right)
$$

Immediately we notice that the signal size is smaller. Incomplete recovery to equilibrium reduces the gain of the signal. During recovery, the TES resistance is $R>R_{0}$. From Eq. 3.24 we would expect the signal size to decrease if $R$ is increased, as shown in Eq. 3.56 . Following a derivation similar to Section 2.6 .3 , we get the probability distribution of pulse height variation due to baseline gain shift

Eq. 3.57P $P\left(I_{\text {Signal }}\right)=n \tau\left(\frac{I_{0}}{2 I_{\text {SignaD }}^{2}}\right)^{n \tau}\left(I_{\text {Signa }}-I_{\text {Signal }}\right)^{m r-1}$, for $\left(1-2 \frac{I_{\text {Signal } 0}}{I_{0}}\right) I_{\text {Signalo }}<I<I_{\text {Signal0 }}$.

This distribution has a similar form as Eq. 2.18, except that the peak broadening tends towards the low energy side of the peak.

\subsection{Current-voltage characteristics}

It is important to understand the current-voltage (IV) characteristics of the TES in its operation. With a good understanding of the IV characteristics, we can extract the experimental value of $G$ and derive the shape of the superconducting transition under actual operating bias. It is customary to plot the voltage $V$ on the $\mathrm{x}$-axis and the current $I$ 
on the y-axis. In an IV plot, radial lines emanating from the origin are lines of constant resistance and hyperbolas of the form $I \sim 1 / V$ are lines of constant power. For this analysis, we assume that the TES has a linear transition from superconducting state to normal state as shown in Fig. 3.7. This simple transition is a reasonable approximation for some microcalorimeters. However, we have observed microcalorimeters with transitions that are non-linear. These devices sometimes have a non-linear response to energy. For the examples here, we use a TES resistance $R(T)$ with a transition at $T_{c}=100$ $\mathrm{mK}$, transition width $\Delta T_{c}=1 \mathrm{mK}$, and normal resistance $R_{n}=1 \Omega$. The TES is coupled to the cold bath with $G=1 \mathrm{nW} / \mathrm{K}$. The resistance of the TES is given by

Eq. 3.58

$$
R(T)=\left\{\begin{array}{cc}
0 & T<T_{c} \\
\frac{R_{n}}{\Delta T_{c}}\left(T-T_{c}\right) & T_{c} \leq T \leq\left(T_{c}+\Delta T_{c}\right) \\
R_{n} & \left(T_{c}+\Delta T_{c}\right)<T
\end{array}\right.
$$

This transition is consistent with the parameters given in Table 3.1. We assume that $R_{T h}$ $\ll R_{n}$ such that TES is in strong voltage bias everywhere except in the superconducting state, where we are under constant current bias.

At temperatures above $T_{c}$, the TES has normal resistance $R_{n}$. The IV curve is a straight line passing through the origin with a slope of $1 / R_{n}$. At temperatures below $T_{c}$, the TES can sustain a small supercurrent less than the critical current. The supercurrent appears as a straight line running along the current axis. The TES does not dissipate any power, therefore, the TES temperature $T=T_{b a t h}$. Fig. 3.8 shows an illustration of an IV curve. 


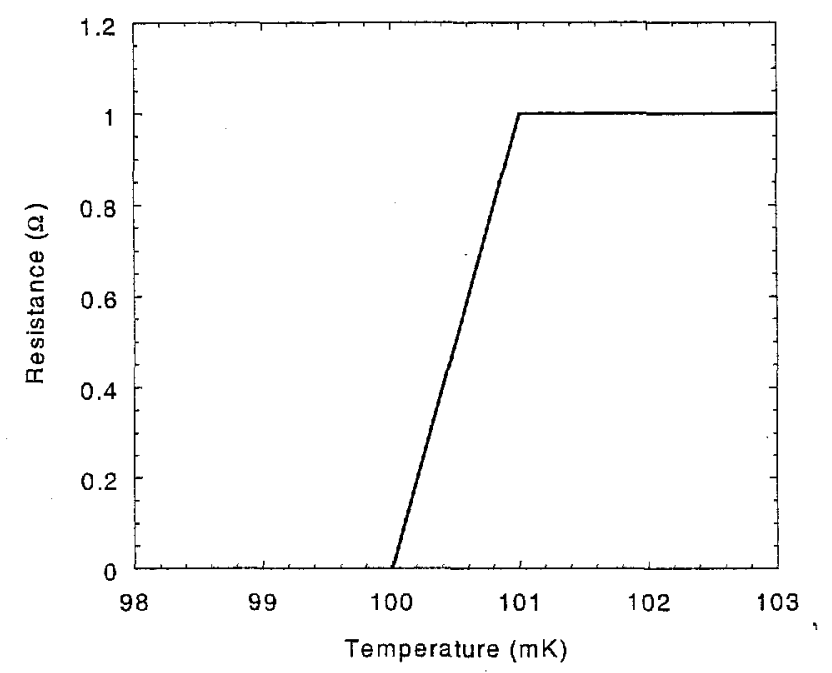

Fig. 3.7 Linear transition from superconducting state to normal state.

If we gradually increase the bias current until the supercurrent equals the critical current (point $a$, Fig. 3.8), the onset of resistance will cause the TES to self heat with Ohmic bias power $P_{\text {Bias }}=I_{c}^{2} R(T)$. The TES is continually cooled with cooling power $P_{\text {cool }}=K\left(T^{N}\right.$ $T_{\text {Bath }}{ }^{N}$ ). Electrothermal equilibrium is reached when $P_{\text {Bias }}=P_{\text {cool }}$. Usually, the self heating will drive the TES into the normal state (point $b$, Fig. 3.8), where it is voltage biased with $V=I_{c} R_{n}$. As we decrease the bias voltage (point $c$, Fig. 3.8), we move along a line of constant resistance for $R_{n}$. The temperature of points along the line is found by equating $P_{\text {bias }}=V^{2} / R_{n}$ with the cooling power, giving $T^{N}=T_{\text {Bath }}{ }^{N}+V^{2} / K R_{n}$. As the bias voltage decreases, the temperature of the TES also decreases. When the TES temperature is less than $T_{c}+\Delta T_{c}$, we enter the transition under voltage bias (point $d$, Fig. 3.8). The resistance of points on the transition is given by $R=V / I$. The corresponding temperature for the points is given by $T^{N}=T_{B a t h}{ }^{N}+V^{2} / K R$. The temperature of points on the transition range from $T_{c}$ to $T_{c}+\Delta T_{c}$. For small $\Delta T_{c}$, points on the transition have approximately the 
same temperature and power. Therefore, the transition appears as a constant power hyperbola on the IV plot. Current and voltage values on the transition follow the relation, $I V=K\left(T^{N}-T_{B a t h}{ }^{N}\right)$. The "distance" of the hyperbola from the origin varies with the bath temperature.

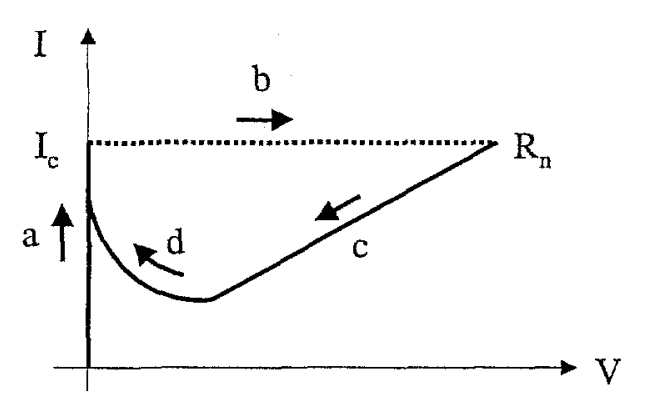

Fig. 3.8 Illustration of IV characteristic.

Because the SQUID read-out of the TES current is a two-wire measurement, there will always be an intrinsic lead resistance in the bias circuit. In the IV plot, lead resistance has the effect of "tilting" the supercurrent to a slope of $1 / R_{\text {lead }}$. In the voltage biased regime of the IV curve, we can derive analytical expressions for $I$ and $V$ as parametric functions of $T$.

Eq. 3.59

$$
V(T)=\sqrt{R(T) K\left(T^{N}-T_{\text {Bath }}^{N}\right)}+I(T) R_{\text {lead }} \quad T_{c}<T,
$$

Eq. 3.60

$$
I(T)=\sqrt{\frac{K\left(T^{N}-T_{\text {Bath }}^{N}\right)}{R(T)}} \quad T_{c}<T,
$$




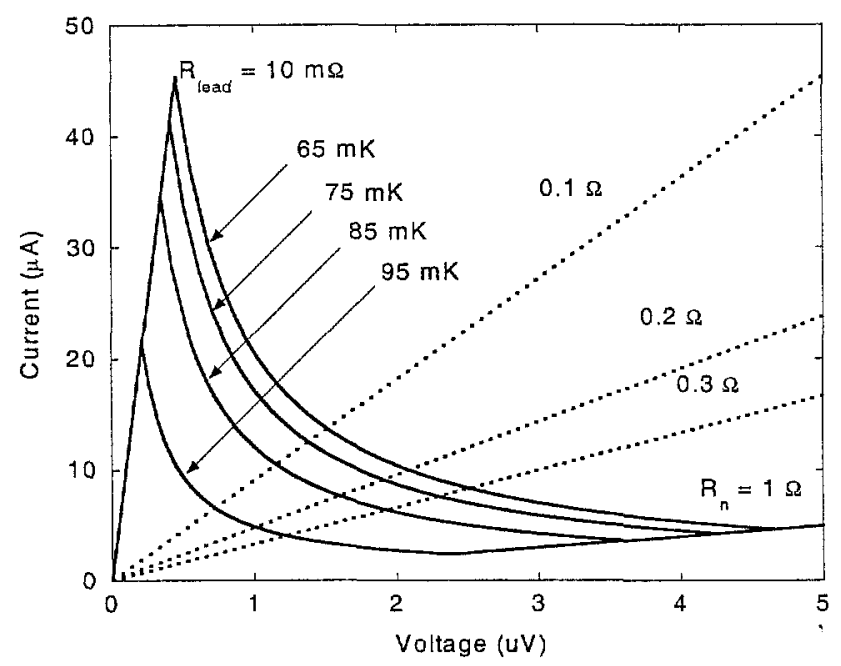

Fig. 3.9 Calculated IV characteristics based on sample TES parameters.

Each IV curve is calculated at a different bath temperature. Dotted lines are lines of constant resistance for $0.1 R_{n}, 0.2 R_{2}, 0.3 R_{n}$.

where $R(T)$ is given by Eq. 3.58 and $R_{\text {lead }}$ is the lead resistance. Fig. 3.9 shows a set of calculated IV curves based on parameters given in Table 3.1 (page 58) and the transition shown in Fig. 3.7 with typical $R_{\text {lead }}=10 \mathrm{~m} \Omega$. Each IV curve is calculated at a different bath temperature.

From the IV curves shown in Fig. 3.9, we can find the bias points that give large signal amplitudes. Using parameters given in Table 3.1, the voltage bias point is located at the intersection of the $0.1 \Omega$ radial line and the $I V$ curve for $T_{\text {bath }}=65 \mathrm{mK}$. For sake of simplicity, we consider the microcalorimeter response for absorption of a $10 \mathrm{keV}$ photon. For the given heat capacity $C=100 \mathrm{keV} / \mathrm{mK}$, a $10 \mathrm{keV}$ photon will raise the TES temperature by $0.1 \mathrm{mK}$, which corresponds to an increase of $0.1 \Omega$ on the transition. The 
resulting negative current pulse would be a downwards vertical excursion from the bias point until it reaches the radial line for $0.2 \Omega$. Then, the current will return to the equilibrium bias point. The vertical distance between the bias point and the radial line for $0.2 \Omega$ is the pulse amplitude. Using the same analysis at different bias points, we see that bias points at high $P_{\text {bias }}$ (i.e., high $I_{0}$ ) and low $R_{0}$ (i.e., high $\alpha$ ) give large signals, as described by Eq. 3.33 .

\section{References}

[1] H.K. Onnes, Comm. Phys. Lab., University of Leyden, 120b, 122b, 124c (1911).

[2] W. Meissner, R. Ochsenfeld, Naturwissenschaften, 21, 787 (1933).

[3] F. and H. London, Proc. Roy. Soc. (London), A149, 71 (1935).

[4] L.N. Cooper, Phys. Rev., 104, 1189 (1956).

[5] J. Bardeen, L.N. Cooper, and J.R. Schrieffer, Phys. Rev., 106, 162 (1957).

[6] M. Tinkham, Introduction to Superconductivity, McGraw-Hill, New York (1996).

[7] C. Kittel, Introduction to Solid State Physics, Wiley \& Sons, New York (1996).

[8] B.D. Josephson, Phys. Lett., 1, 251 (1962).

[9] B.D. Josephson, Adv. Phys., 14, 419 (1965).

[10] J.S. Langer, V. Ambegaokar, Phys. Rev., 164, 498 (1967).

[11] C.M. Knoedler, Journ. Appl. Phys., 54(5), 2773 (1983).

[12] M.A. Lindeman, Ph.D. Dissertation, University of California, Davis (2000).

[13] P. Horowitz, W. Hill, The Art of Electronics, Cambridge (1989). 
[14] F. Pröbst, M. Frank, S. Cooper, P. Colling, D. Dummer, P. Ferger, G. Forster, A. Nuciotti, W. Seidel, L. Stodolsky, Journ. Low Temp. Phys., 100(1/2), 65 (1995).

[15] S.H. Moseley, J.C. Mather, D. McCammon, J. Appl. Phys., 56 (5), 1257 (1984).

[16] K.D. Irwin, Ph.D. Dissertation, Stanford University (1995).

[17] K.D. Irwin, Appl. Phys. Lett., 66(15), 1998 (1995).

[18] A.E. Szymkowiak, R.L. Kelly, S.H. Moseley, C.K. Stahle, Journ. Low Temp. Phys., 93(3-4), 281 (1993).

[19] J.M. Gildemeister, Ph.D. Dissertation, University of California, Berkeley (2000). 
Chapter 4

\section{DETECTOR DESIGN}

In the following sections we will discuss the performance requirements of the microcalorimeter and design issues of each component. The basic design of the microcalorimeter is as follows. The absorber is $99.995 \%$ high-purity bulk $\mathrm{Sn}$. The TES is a $\mathrm{Mo} / \mathrm{Cu}$ multilayer superlattice. The substrate is a silicon nitride (SiN) membrane window supported by a silicon substrate frame. The silicon substrate acts as the cold bath. The SiN membrane serves as the weak thermal link between the TES and the cold bath. The absorber is coupled to the TES film with a microscopic amount of epoxy. Fig. 4.1 shows an illustration of the microcalorimeter design.

\subsection{Absorber}

For gamma ray spectroscopy, the absorber is generally the largest component of the detector. For good absorption efficiency, the material should have high $Z$ and be physically thick. The absorber must also have a large collection area to maximize count rate. But at the same time, the material needs to have a small heat capacity to increase sensitivity and shorten time scales. 


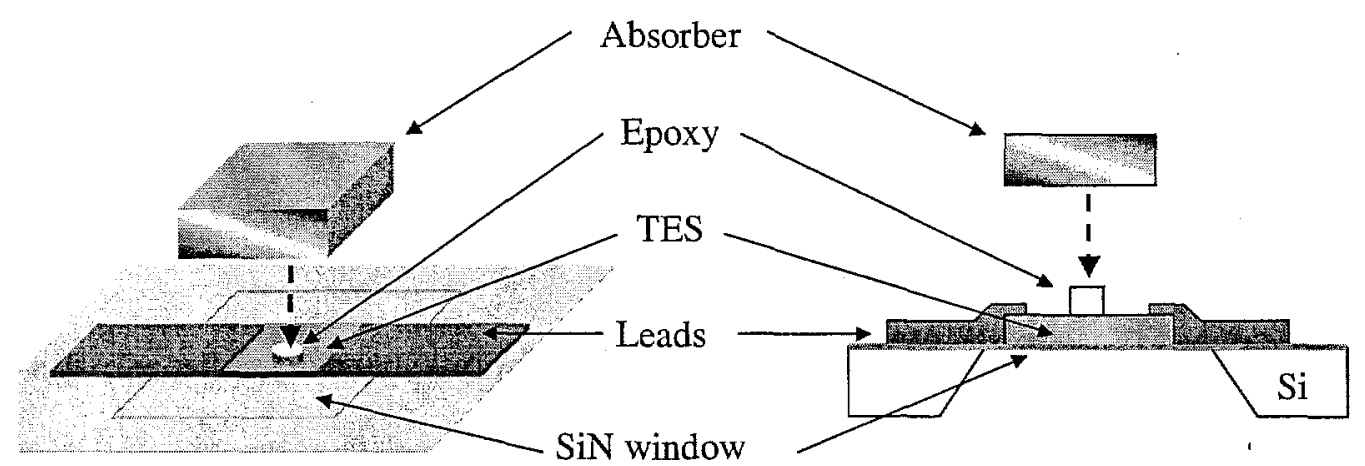

Fig. 4.1 Illustration of microcalorimeter design in 3D and cross sectional views.

\subsubsection{Material choices}

Elemental superconductors satisfy these requirements. Many are transition metals, with relatively high stopping power. For an operating temperature of the microcalorimeter $T_{0}$ $<T_{c}$, the heat capacity of the superconductor is dominated by the phonon heat capacity, which is typically three orders of magnitude smaller than the electronic heat capacity for the same material. Some suitable materials include $\mathrm{Ta}\left(Z=73, T_{c}=4.48 \mathrm{~K}, \theta_{D}=240 \mathrm{~K}\right)$, $\operatorname{Re}\left(Z=75, T_{c}=1.4 \mathrm{~K}, \theta_{D}=430 \mathrm{~K}\right), \mathrm{Mo}\left(Z=42, T_{c}=0.92 \mathrm{~K}, \theta_{D}=450 \mathrm{~K}\right)$, and $\mathrm{Pb}(Z=$ $\left.82, T_{c}=7.19 \mathrm{~K}, \theta_{D}=105 \mathrm{~K}\right)$. However, it has been observed that these materials tend to have metastable states which prevent complete thermalization of photon energy [1][2].

Thermalization efficiency in superconducting materials has been studied extensively[3]. For all materials at temperatures $T<3 \times 10^{-4} \theta_{\mathrm{D}}, 100 \%$ of the incident energy was thermalized in a short time. At lower temperatures, the fraction of thermalized energy decreases sharply. Other research groups have found $\operatorname{Sn}\left(Z=50, T_{c}=3.77, \theta_{D}=200 \mathrm{~K}\right)$ 
to be a good absorber without thermalization problems [4][5][6]. Therefore, we choose to use high purity polycrystalline bulk $\mathrm{Sn}$ as our absorber.

\subsubsection{Absorption efficiency}

We purchased commercially available high purity $(99.995 \%)$ rolled Sn foil with a thickness of $250 \mu \mathrm{m}$. For high energy gamma ray detection, the absorber needs to be much thicker. However, for prototyping purposes, $250 \mu \mathrm{m}$ is sufficient. Fig. 4.2 shows the calculated absorption efficiency for $250 \mu \mathrm{m}$ thick Sn based on known attenuation coefficients [7]. This Sn has nearly $100 \%$ absorption up to tens of keV. For $60 \mathrm{keV}$ gamma rays, the absorption efficiency is approximately 54\%. The dip near $25 \mathrm{keV}$ corresponds to the $\mathrm{K}$ absorption edge of $\mathrm{Sn}$.

\subsubsection{Heat capacity}

The phonon heat capacity of $\mathrm{Sn}$ is given by Eq. 3.14 , with

Eq. 4.1

$$
\alpha_{S n}=100.22 \frac{\mathrm{eV}}{\mu \mathrm{m}^{3} \mathrm{~K}^{4}} .
$$

For a $1 \mathrm{~mm} \times 1 \mathrm{~mm} \times 0.25 \mathrm{~mm}$ volume of bulk $\mathrm{Sn}$, the heat capacity at $100 \mathrm{mK}$ is 25 $\mathrm{keV} / \mathrm{mK}(4.01 \mathrm{pJ} / \mathrm{K})$. 


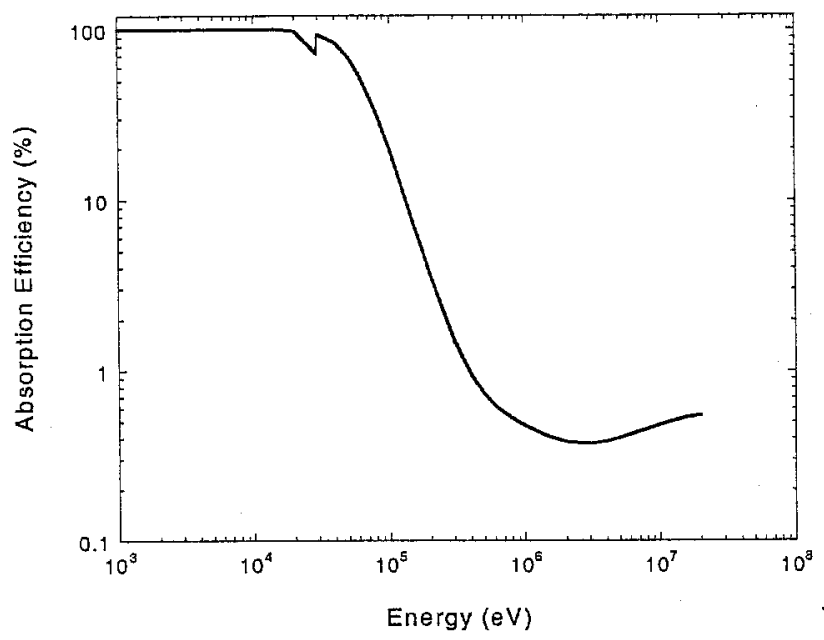

Fig. 4.2 Calculated absorption efficiency for $250 \mu \mathrm{m}$ thick Sn

\subsection{Superconducting transition-edge sensor}

\subsubsection{Proximity Effect}

The TES is a thin film device. No elemental superconductor has a transition temperature at our desired operating temperature. We take advantage of the proximity effect to choose our transition temperature. If a superconducting film is in close proximity with a normal metal film such that Cooper pairs diffuse into the normal metal, the normal metal becomes weakly superconducting [8]. If the thicknesses of the two films are less than the coherence length of the superconductor, then the normal metal is complete proximitized and the bilayer behaves as a superconductor with properties different from the superconducting component film. In essence, the composite film alters the electron density such that, according to Eq. 3.7, the transition temperature and energy gap is different. In our early experiments, we used an Al/Cu bilayer as the TES [9][10][11]. 
However, $\mathrm{Al}$ and $\mathrm{Cu}$ were highly miscible. The layers interdiffused, causing $T_{c}$ to drift in an apparently random manner with time.

More recent TES films used $\mathrm{Mo} / \mathrm{Cu}$ multilayer superlattices. A multilayer structure consists of many alternating layers of $\mathrm{Mo}$ and $\mathrm{Cu}$. $\mathrm{Mo}$ and $\mathrm{Cu}$ are mutually immiscible, making the films stable. The transition temperature depends on the thickness ratio of each $\mathrm{Mo}$ and $\mathrm{Cu}$ layer. The multilayer structure allows scaling in thickness by addition of more layers. This allows us to control the volume, heat capacity, and resistivity of the film. We may even construct thick films so that low energy $\mathrm{x}$ rays may be absorbed directly in the TES without the aid of an absorber. Furthermore, $\mathrm{Mo} / \mathrm{Cu}$ films are stable against annealing to remove interstitial gases. Fig. 4.3 shows experimental data for transition temperature $T_{c}$ as a function of the thickness of $\mathrm{Cu}$ while keeping the thickness of Mo constant at $2 \mathrm{~nm}$ [12]. We see a negative exponential dependence as we vary the thickness of $\mathrm{Cu}$, as expected from Eq. 3.7. For a transition temperature of $100 \mathrm{mK}$, the thickness ratio $\mathrm{Mo} / \mathrm{Cu}: 2 \mathrm{~nm} / 7 \mathrm{~nm}$.

\subsubsection{Temperature stability}

For the TES to be a sensitive thermometer, the film must be uniform in temperature and have good thermal conductivity within itself. The Wiedemann-Franz law relates thermal conductivity to electrical resistance, therefore limits must exists for the normal state resistance of the TES for good temperature stability. The Wiedemann-Franz law is 


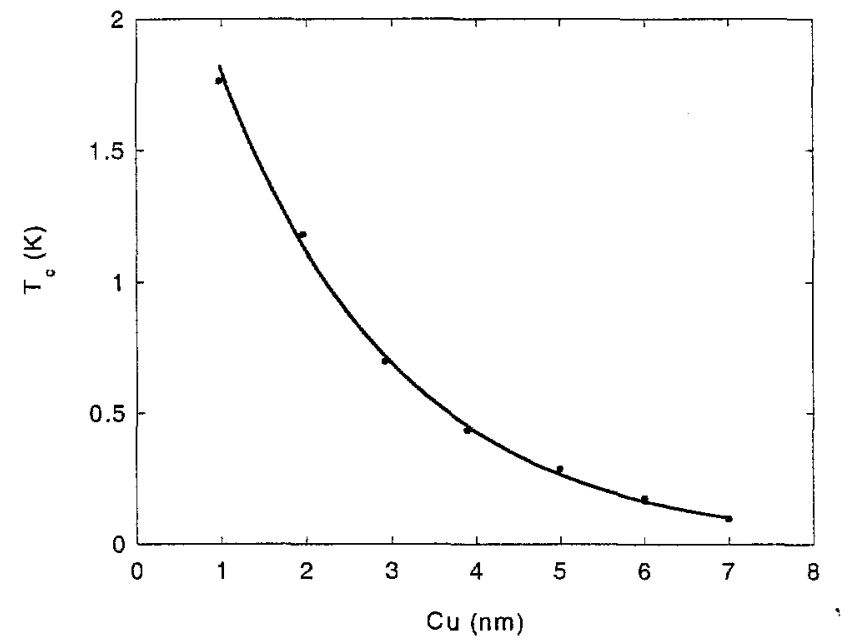

Fig. 4.3 Multilayer transition temperature vs. Cu thickness for fixed Mo thickness of $2 \mathrm{~nm}$.

Eq. 4.2

$$
\kappa=\frac{L T}{\rho_{n}}
$$

where $\kappa$ is thermal conductivity of the TES film, $\rho_{n}$ is the resistivity of the film in the normal state, and $L$ is the Lorenz number. The theoretical value of $L$ is $2.45 \times 10^{-8}$ $\mathrm{W} \Omega / \mathrm{K}^{2}$.

A calculation can be performed for the simple theoretical case of a one-dimensional TES, where the width of the TES is less than the coherence length. The temperature spatial profile of the TES is expressed by the differential equation

Eq. 4.3

$$
C \frac{d T}{d t}=-K \cdot\left(T^{N}-T_{B a t h}^{N}\right)+J^{2} \rho(T(x))-\kappa \frac{\partial^{2} T}{\partial x},
$$


where $J$ is the current density, and $C$ and $G$ are the heat capacity and thermal conductivity per unit length in this case [11]. The first term on the right side is the cooling of the TES to the substrate. The second term is the Ohmic heating. The third term is the thermal conduction within the TES. It has been shown [13][11] that the TES film is stable when

Eq. $4.4 \quad R_{n}<\frac{\pi^{2} L T}{G \alpha}$.

A realistic value for the thermal conductivity of the SiN membrane is $G_{S i N} \sim 0.5 \mathrm{nW} / \mathrm{K}$ (Section 4.3, page 80). Assuming $T_{0}=100 \mathrm{mK}$ and $\alpha=100$, the upper limit for the normal state resistance of the TES is $\sim 0.5 \Omega$. However, the Lorenz number is material dependent and also tends to decrease at low temperatures $[14][15]$, so Eq. 4.4 is only a crude approximation.

\subsubsection{Electrons and phonons}

In a metals at low temperatures, electrons and phonons are considered as separate systems with their own distinct temperatures. The electrons and phonons interact via scattering. At room temperatures, the thermal conductivity between electrons and phonons is very strong, so the electrons and phonons have nearly identical temperatures. However, at low temperatures, the thermal conductivity decreases. The two systems decouple and may attain different equilibrium temperatures. The power flow between the electron and the phonon systems is given by 
Eq. 4.5

$$
P_{e-p}=\Sigma V_{T E S}\left(T_{e T E S}^{5}-T_{p T E S}^{5}\right)
$$

where $V$ is the volume of the film, $T_{e T E S}$ and $T_{p T E S}$ are the respective temperatures of the electrons and phonons of the TES. The parameter $\Sigma$ for $\mathrm{Cu}$ is measured by Wellstood to be $(2.4 \pm 0.6) \times 10^{9} \mathrm{~W} / \mathrm{m}^{3} \mathrm{~K}^{5}[16]$. Roukes measured $\Sigma$ to be $1.8 \times 10^{9} \mathrm{~W} / \mathrm{m}^{3} \mathrm{~K}^{5}[17]$ and Anderson found $1.0 \times 10^{9} \mathrm{~W} / \mathrm{m}^{3} \mathrm{~K}^{5}[18]$. The thermal conductivity between phonons and electrons is

$\mathrm{Eq} \cdot 4.6$

$$
G_{e-p}=\frac{d P}{d T}=5 \Sigma V_{T E S} T^{4}
$$

In a TES, the superconducting transition is an effect in the electronic system. The cooling to the SiN membrane, however, is a phonon effect. At $100 \mathrm{mK}$, the electronphonon coupling is $G_{e-p}=120 \mathrm{nW} / \mathrm{K}$, for the TES operating parameters given in Table 3.1 and the dimensions given in the next section. The TES electrons must dissipate $P_{\text {Bias }}$ $=40 \mathrm{pW}$ into the phonon system. For the electron temperature $T_{e}=T_{0}=100 \mathrm{mK}$, the phonon temperature is $T_{p}=99.67 \mathrm{mK}$, which is distinctively different.

\subsubsection{Heat capacities}

Since the electron and phonon systems must be regarded separately, we need to determine the electron and phonon heat capacities for the TES. Electron heat capacity near the superconducting transition is given by Eq. 3.13, where, for $\mathrm{Cu}$ and $\mathrm{Mo}$, respectively, 
Eq. 4.7

$$
\gamma_{C u}=607 \frac{\mathrm{eV}}{\mu \mathrm{m}^{3} \cdot \mathrm{K}^{2}}, \gamma_{M o}=1217 \frac{\mathrm{eV}}{\mu \mathrm{m}^{3} \cdot \mathrm{K}^{2}}
$$

Phonon heat capacity is given by Eq. 3.14, where, again, for $\mathrm{Cu}$ and Mo, respectively,

Eq. 4.8

$$
\alpha_{C u}=41.724 \frac{\mathrm{eV}}{\mu \mathrm{m}^{3} \cdot \mathrm{K}^{4}}, \alpha_{M o}=15.674 \frac{\mathrm{eV}}{\mu \mathrm{m}^{3} \cdot \mathrm{K}^{4}}
$$

Our multilayer TES consists of 20 layers of $2 \mathrm{~nm}$ thick Mo and 23 layers of $7 \mathrm{~nm}$ thick Cu. The TES dimensions are $1 \mathrm{~mm} \times 0.5 \mathrm{~mm}$ and $200 \mathrm{~nm}$ thick. The total electronic heat capacity at $100 \mathrm{mK}$ is $17.6 \mathrm{keV} / \mathrm{mK}$. The total phonon heat capacity is $3.67 \mathrm{eV} / \mathrm{mK}$, which is nearly 5000 times smaller than the electronic heat capacity.

\subsubsection{Electrical leads}

Bias to the TES is applied via superconducting Al leads. The leads run from the TES over the membrane to the silicon substrate, where they are wired to the bias circuit. The leads provide a thermal conduction path that is parallel to the SiN membrane. The thermal conductivity for superconducting $\mathrm{Al}$ is given by

Eq. 4.9

$$
G_{A l}=\kappa_{A l} \frac{A_{A l}}{L_{A l}}
$$

with

$$
\kappa_{A l}=\frac{1}{3} C_{A l} v_{A l} l_{A l},
$$


where $C_{A l}$ is the phonon heat capacity per unit volume of $\mathrm{Al}$ given by Eq. 3.14 with $\alpha_{A l}=$ $15.67 \mathrm{eV} / \mu \mathrm{m}^{3} \mathrm{~K}^{4} ., v_{A l}=3428 \mathrm{~m} / \mathrm{s}$ is the speed of sound in $\mathrm{Al}[19], l_{A l}$ is the phonon mean free path in $\mathrm{Al}$, and $A_{A l}$ and $L_{A l}$ are the cross sectional area and length of the heat flow. The Al leads are deposited on top of the TES and need to be at least at thick as the TES for good step coverage. The effective length of the Al leads is $100 \mu \mathrm{m}$, which is the distance between the edge of the TES and the silicon substrate. Assuming the phonon mean free path in $\mathrm{Al}$ is at most as thick as the film, and for $\mathrm{Al}$ leads that are $200 \mathrm{~nm}$ thick and $500 \mu \mathrm{m}$ wide, the thermal conductivity at $100 \mathrm{mK}$ is $\mathrm{G}_{\mathrm{Al}}=0.2 \mathrm{pW} / \mathrm{K}$. The Al leads also overlap the TES by $\sim 250 \mu \mathrm{m}$ for each lead. This defines the effective area of the TES to be $500 \mu \mathrm{m} \times 500 \mu \mathrm{m}$ square with a measured typical $R_{n} \sim 0.5 \Omega$. The total length and width of the leads are not critical as long as the current density is small such that the leads will not be driven normal during operation.

\subsection{SiN membrane}

The TES is fabricated on a silicon nitride ( $\mathrm{SiN}$ ) membrane supported by a silicon frame. The SiN membrane provides thermal isolation and serves as the weak thermal link between the TES and the cold bath. The thermal conductivity of the SiN membrane has been well characterized [20]. The power flow through the SiN membrane can be written as

Eq. 4.10

$$
P_{S i N}=K_{S i N}\left(T_{\text {eTES }}^{4}-T_{B a t h}^{4}\right)
$$

with thermal conductivity 
Eq. 4.11

$$
G_{S i N}=\frac{d P_{S i N}}{d T}=4 K_{S i N} T^{3}
$$

where $K_{S i N}=\sigma A \xi, A$ is the cross sectional area of the membrane perpendicular to the direction of heat flow, and $\sigma=\Sigma_{\mathrm{i}}\left(\pi^{5} k^{4} / 15 h^{3} v_{i}^{2}\right)=15.7 \mathrm{~mW} / \mathrm{cm}^{2} \mathrm{~K}^{4}$ is the Stefan-Bolzmann constant obtained by summing over the transverse and longitudinal acoustic modes. This thermal conductivity differs from the usual form for heat flow through a solid (eg., Eq. 4.9) which depends on the distance of heat flow. It assumes that thermal energy is conducted via radiation of phonons into the membrane. Within the membrane volume, the phonons may scatter. The prefactor $\xi$ accounts for the reduction in thermal conductivity due to diffuse scattering of phonons. For specular, or "clean" scattering, $\xi=$ 1. For diffuse, or "dirty", scattering, $\xi=3 l_{\mathrm{Ph}} / 4 L_{S i N} \ll 1$, where $l_{\mathrm{Ph}}$ is the phonon mean free path and $L_{S i N}$ is the distance through the $\mathrm{SiN}$ the heat must flow [21].

At low temperatures, phonon wavelengths may exceed the thickness of the TES film. The phonon wavelength is $\lambda \sim \hbar v / k T$, where $\nu \sim 10^{4} \mathrm{~m} / \mathrm{s}$ is the speed of sound in the membrane [20]. At $100 \mathrm{mK}$, the average phonon wavelength is $\lambda \sim 1 \mu \mathrm{m}$. Therefore, the TES at $100 \mathrm{mK}$ with a thickness of $200 \mathrm{~nm}$ does not support a phonon system that is distinctly separate from the SiN membrane. As a result, the TES and the membrane share phonons, eliminating any Kapitza boundary resistance between the two [16].

SiN membranes available for our detector has a window size of $1.2 \mathrm{~mm} \times 1.2 \mathrm{~mm}$ and a thickness of $0.5 \mu \mathrm{m}$. Since the membrane has a temperature gradient of $T_{0}=100 \mathrm{mK}$ 
directly under the TES to $T_{b a t h}=65 \mathrm{mK}$ over the silicon substrate, we estimate the average membrane temperature to be $T_{S i N}=82.5 \mathrm{mK}$, which is half way between the two. Using the perimeter of the TES and the thickness of the membrane as the cross sectional area, the thermal conductivity for the membrane is $G_{S i N}=0.529 \mathrm{nW} / \mathrm{K}$. Compared with the parallel conduction path of the $\mathrm{Al}$ leads, $G_{S i N}$ is over three orders of magnitude greater than $G_{A l}$. Therefore, the dominant cooling pathway is through the SiN membrane.

\subsection{Stycast 2850FT epoxy}

The Sn absorber is coupled to the TES by a miniscule amount of Stycast 2850FT epoxy. Stycast is a standard material in cryogenic experiments with properties that are well documented [22][23].

\subsubsection{Heat capacity}

The heat capacity is assumed to have the form [22]

Eq. 4.12

$$
C_{S t y}=\left(B_{1} T+B_{3} T^{3}+B_{5} T^{5}\right) \rho_{S t y} V_{S t y}
$$

with coefficients

Eq. 4.13

$$
B_{1}=7 \times 10^{-6} \frac{\mathrm{J}}{\mathrm{g} \cdot \mathrm{K}^{2}}, B_{3}=4.56 \times 10^{-6} \frac{\mathrm{J}}{\mathrm{g} \cdot \mathrm{K}^{4}}, B_{5}=1.67 \times 10^{-6} \frac{\mathrm{J}}{\mathrm{g} \cdot \mathrm{K}^{6}},
$$


and density $\rho_{S t y}=2.4 \mathrm{~g} / \mathrm{cm}^{3}$, and volume $V_{S t y}$. For a volume of Stycast $200 \mu \mathrm{m}$ in diameter and $25 \mu \mathrm{m}$ thick at $100 \mathrm{mK}$, the heat capacity is $C_{S t y}=8.29 \mathrm{keV} / \mathrm{mK}$.

\subsubsection{Boundary resistance}

The Stycast forms an interface with both $\mathrm{Sn}$ absorber and the TES film. There is an associated Kapitza resistance at these interfaces. We take the typical value for the thermal conductivity of a metal-epoxy interface [24],

Eq. 4.14

$$
G_{K}=\left(1.6 \frac{k W}{m^{2} \cdot K^{4}}\right) T^{3} A
$$

where $A$ is the contact area. For Stycast of diameter $200 \mu \mathrm{m}$, the thermal conductivity is $G_{K}=50 \mathrm{nW} / \mathrm{K}$, which is 100 times greater than $G_{S i N}$. The thermal conductivity of the Stycast limits the rate at which energy can flow from the absorber to the TES. This rate is slower than the thermalization time of the absorber, thus effectively forming a bottle neck.

\subsubsection{Thermal conductivity}

There is a finite thermal conductivity through the bulk volume of the Stycast as well. This has been measured and expressed in the usual form as [23]

Eq. 4.15

$$
G_{S t y}=\kappa_{S t y} \frac{A_{S t y}}{L_{S t y}},
$$

with

$$
\kappa_{S t y}=a T^{n},
$$


where $a=92 \mu \mathrm{W} / \mathrm{cm} \mathrm{K}, n=2.65$, and $A_{A l}$ and $L_{A l}$ are the cross sectional area and length of the heat flow. For Stycast with the above dimensions, the bulk thermal conductivity is $G_{S t y}=25.9 \mathrm{nW} / \mathrm{K}$, which is comparable to $G_{K}$.

We may include the bulk thermal conductivity of the Stycast into the boundary resistance by writing an effective Kapitza coupling

Eq. 4.16

$$
\frac{1}{G_{K(e f f)}}=\frac{1}{G_{K}}+\frac{1}{2 G_{S t y}}
$$

where we only consider the thermal conductivity of half the thickness of Stycast to be associated with each interface.

With an understanding of the physical properties associated with each component of the microcalorimeter, we can begin to develop an understanding of its performance. 


\section{References}

[1] M.L. van den Berg, D.T. Chow, A. Loshak, M.F. Cunningham, T.W. Barbee, Jr., M. Frank, S.E. Labov, Proc. SPIE, 4140, 436 (2000).

[2] C.K. Stahle, Ph.D. Dissertation, Stanford University (1991).

[3] S. Vitale, G. Gallinaro, F. Gatti, Proc. SPIE, 1743, 368 (1992).

[4] E. Silver, M. LeGros, G. Austin, N. Madden, J. Beeman, E. Haller, X-Ray Spectrom., 25, 265 (1997).

[5] A. Alessandrello, J. W. Beeman, C. Brofferio, O. Cremonesi, E. Fiorini, A. Giuliani, E. E. Haller, A. Monfardini, A. Nucciotti, M. Pavan, G. Pessina, E. Previtali, L. Zanotti, Phys. Rev. Lett., 82, 513 (1999).

[6] A. Bleile, P. Egelhof, H. J. Kluge, U. Liebisch, D. McCammon, H. J. Meier, O. Sebastian, C. K. Stahle, M. Weber, Nucl. Instr. Meth. A, 444, 488 (2000).

[7] D.C. Creagh, J.H. Hubbell, International Tables for Crystallography, Kluwer, Dordrecht (1992).

[8] L.N. Cooper, Phys. Rev. Lett., 6, 689 (1961).

[9] D. Chow, APS Centennial Meeting, Session YC07.8, Atlanta (1999).

[10] H. Netel, Ph.D. Dissertation, Universiteit Twente (1999).

[11] M.A. Lindeman, Ph.D. Dissertation, University of California, Davis (2000).

[12] M.F. Cunningham, M.A. Lindeman, D.T. Chow, M. Frank, T.W. Barbee, Jr., S.E. Labov, to be published in Nucl. Instr. Meth. (2000). 
[13] K.D. Irwin, G.C. Hilton, D.A. Wollman, et al., Journ. Appl. Physics, 83(8), 3978 (1998).

[14] C. Kittel, Introduction to Solid State Physics, Wiley \& Sons, New York (1996).

[15] J. Ziman, Electrons and phonons, Oxford (1960).

[16] F.C. Wellstood, C. Urbina, J. Clarke, Phys. Rev. B, 49, 5942 (1994)

[17] M.L. Roukes, M.R. Freeman, R.S. Germain, R.C. Richardson, M.B. Ketchen, Phys. Rev. Lett., 55, 422 (1985).

[18] P.W. Anderson, E. Abrahams, T.V. Ramakrishnan, Phys. Rev. Lett., 43, 719 (1979).

[19] S.B. Kaplan, Journ. Low Temp. Phys., 37, 343 (1979).

[20] W. Holmes, J.M. Gildemeister, P.L. Richards, Appl. Phys. Lett., 72, 2250 (1998).

[21] M. Wyebourne, C. Eddison, M. Kelly, J. Phys. C, 17, L607 (1984).

[22] C.A. Swenson, Rev. Sci. Instrum., 68(2), 1312 (1997).

[23] J.R. Olson, Cryogenics, 33, 729 (1970).

[24] A.C. Anderson, R.E. Peterson, Cryogenics, 10, 430 (1970). 
Chapter 5

\section{NUMERICAL MODEL}

\subsection{Default parameters}

With the properties of the detector components described in the previous chapter, we can model the response of the microcalorimeter. With the numerical model, we can vary each physical property and optimize the signal-to-noise ratio. Table 5.1 is a list of all relevant parameters and starting values that enter the numerical model of the TES. All numerical models will use these "default" values, unless specified otherwise. Most numerical calculations are performed with MathCAD analysis software.

\subsection{Current-voltage characteristics}

The first measurement in the any experimentation of TES devices is the IV characteristics. For our model, we choose to use the simple linear transition given by Eq. 3.58 except with a normal state resistance given in Table 5.1 of $0.5 \Omega$, as required for temperature spatial uniformity. Following the formulation provided in Section 3.8 (page 
64), we derive a set of IV curves (Fig. 5.1) based on the default parameters at different bath temperatures.

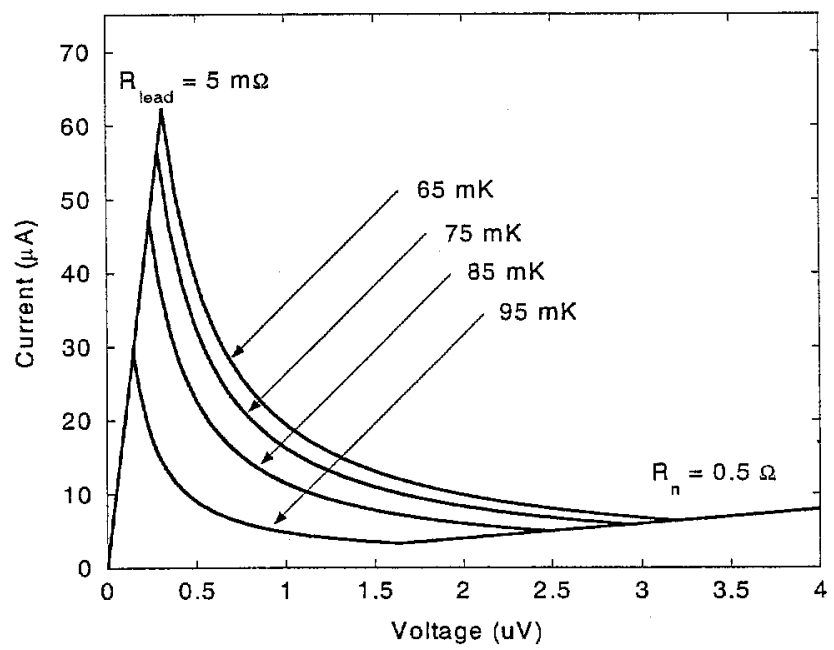

Fig. 5.1 Modeled IV curves based on default parameters

\subsection{Multi-element numerical model}

From the discussion in Chapter 4, we recognize our microcalorimeter design to consist of four distinct components: Sn absorber, Stycast epoxy, TES phonons, and TES electrons as shown in Fig. 5.2.

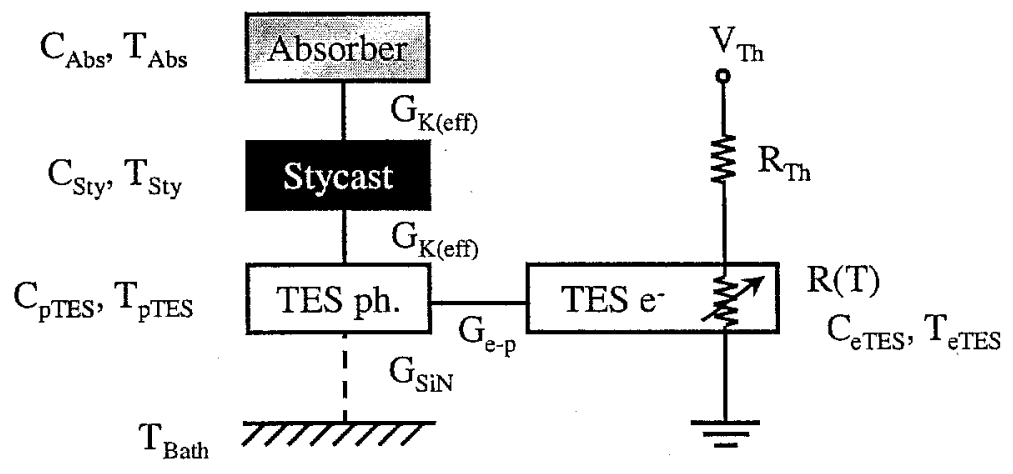

Fig. 5.2 Thermal diagram of the 4-component microcalorimeter. 


\begin{tabular}{|c|c|c|c|}
\hline & Parameter & Description & Value \\
\hline \multirow{6}{*}{ 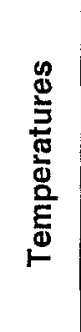 } & $T_{0}$ & Operating & $100.2 \mathrm{mK}$ \\
\hline & $T_{T h}$ & Bias resistor & $2 K$ \\
\hline & $T_{\text {Bath }}$ & Bath & $65 \mathrm{mK}$ \\
\hline & $\Delta T_{\text {Bath }}$ & Fluctuation in bath & $10 \mu \mathrm{K}$ \\
\hline & $T_{C}$ & Transition & $100 \mathrm{mK}$ \\
\hline & $\Delta T_{C}$ & Transition width & $1 \mathrm{mK}$ \\
\hline \multirow{3}{*}{$\begin{array}{l}\stackrel{g}{g} \\
\stackrel{g}{\Xi} \\
\frac{0}{\rho}\end{array}$} & $V_{A b s}$ & Sn absorber & $1 \mathrm{~mm} \times 1 \mathrm{~mm} \times 0.25 \mathrm{~mm}$ \\
\hline & $V_{\text {Sty }}$ & Stycast epoxy & $200 \mu \mathrm{m} \varnothing \times 25 \mu \mathrm{m}$ \\
\hline & $V_{\text {TES }}$ & TES & $1 \mathrm{~mm} \times 0.5 \mathrm{~mm} \times 200 \mathrm{~nm}$ \\
\hline \multirow{4}{*}{ 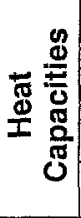 } & $C_{A b s}$ & Sn absorber & $25.2 \mathrm{keV} / \mathrm{mK}$ \\
\hline & $C_{\text {Sty }}$ & Stycast epoxy & $8.31 \mathrm{keV} / \mathrm{mK}$ \\
\hline & $C_{\text {eTES }}$ & TES electron & $17.6 \mathrm{keV} / \mathrm{mK}$ \\
\hline & $C_{\text {pTES }}$ & TES phonons & $3.69 \mathrm{eV} / \mathrm{mK}$ \\
\hline \multirow{5}{*}{ F } & $G_{K}$ & Kapitza interface & $50.6 \mathrm{nW} / \mathrm{K}$ \\
\hline & $G_{\text {Sty }}$ & Stycast epoxy & $26.0 \mathrm{nW} / \mathrm{K}$ \\
\hline & $G_{e-p}$ & TES electron-phonon & $121.6 \mathrm{nW} / \mathrm{K}$ \\
\hline & $G_{\text {SiN }}$ & SiN membrane & $0.531 \mathrm{nW} / \mathrm{K}$ \\
\hline & $G_{K(\text { efi })}$ & Effective Kapitza & $25.4 \mathrm{nW} / \mathrm{K}$ \\
\hline \multirow{4}{*}{$\begin{array}{c}y \\
\mathbb{d} \\
\frac{\pi}{\pi} \\
\frac{w}{y} \\
\frac{d}{x}\end{array}$} & $R_{T h}$ & Thévenin bias & $10 \mathrm{~m} \Omega$ \\
\hline & $R_{n}$ & TES (normal) & $0.5 \Omega$ \\
\hline & $R_{0}$ & TES (operating) & $0.1 \Omega$ \\
\hline & $R_{\text {lead }}$ & Lead & $5 \mathrm{~m} \Omega$ \\
\hline \multirow{4}{*}{$\underset{⿱ 亠 䒑}{\tilde{D}}$} & $V_{T h}$ & Bias voltage & $1.5 \mu \mathrm{V}$ \\
\hline & 10 & Steady-state current & $13.67 \mu \mathrm{A}$ \\
\hline & $P_{0}$ & Bias power & $18.7 \mathrm{pW}$ \\
\hline & $\alpha$ & Transition "steepness" & 500 \\
\hline \multirow{2}{*}{ 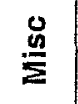 } & $E$ & Gamma ray energy & $60 \mathrm{keV}$ \\
\hline & $I_{\text {SQUIO }}$ & SQUID noise & $10 \mathrm{pA} / \mathrm{Hz}^{1 / 2}$ \\
\hline
\end{tabular}

Table 5.1 Default values for numerical model. 
Following Pröbst, et al. [1], we can describe the temperature evolution of the four components with a set of four first-order differential equations,

Eq. 5.1

$$
\begin{aligned}
& C_{A b s} \frac{d}{d t} T_{A b s}=-G_{K(e f f)}\left(T_{A b s}-T_{S y y}\right) \\
& C_{S t y} \frac{d}{d t} T_{S t y}=G_{K(e f f)}\left(T_{A b s}-T_{S t y}\right)-G_{K(e f f)}\left(T_{S t y}-T_{p T E S}\right)
\end{aligned}
$$

$$
\begin{aligned}
& C_{p T E S} \frac{d}{d t} T_{p T E S}=G_{K(e f f)}\left(T_{S t y}-T_{p T E S}\right)-G_{e-p}\left(T_{p T E S}-T_{e T E S}\right)-K_{S i N}\left(T_{p T E S}^{N}-T_{B a t h}^{N}\right) \\
& C_{e T E S} \frac{d}{d t} T_{e T E S}=-G_{e p}\left(T_{e T E S}-T_{p T E S}\right)+\frac{V_{T h}^{2} R(T)}{\left(R_{T h}+R(T)\right)^{2}}
\end{aligned}
$$

where $T_{A b s}, T_{S t y}, T_{p T E S}$, and $T_{\text {eTES }}$ are the temperatures of the absorber, Stycast, TES phonons, and TES electrons, respectively. The terms of the differential equations have units of power. Generally, power flow between two objects is given by the general form

$$
P=K\left(T_{1}^{N}-T_{2}^{N}\right)
$$

For $T_{1} \approx T_{2}$, we can Taylor expand this expression about $T_{0}+\Delta T$, where $T_{0}=T_{1} \approx T_{2}$ and $\Delta T=T_{1}-T_{2}$

Eq. 5.3

$$
P=G \Delta T+\cdots,
$$

where $G=N K T_{0}^{N-1}$. For our microcalorimeter, $T_{A b s}, T_{S t y}, T_{p T E S}$, and $T_{e T E S}$ have similar values near $T_{0}=100 \mathrm{mK}$. Therefore, the first-order approximation given by Eq. 5.3 is appropriate to describe power flow between these components. However, $T_{p \text { TES }}$, and $T_{B a t h}$ 
are far apart $(\sim 35 \mathrm{mK})$, thus requiring the use of Eq. 5.2 to describe the power flow to the cold bath. For the cooling power to the cold bath, the difference between Eq. 5.3 and Eq. 5.2 is $\sim 4.5 \%$, using the default parameters given in Table 5.1 .

The steady state solutions of the differential equations can be found easily. The TES electrons has the highest temperature, $T_{\text {eTES }}=T_{0} \approx T_{\text {Bath }}+\left(P_{0} / G_{S i N}\right)$. The temperature of the TES phonons, Stycast, and absorber are all the same, which is cooler than $T_{\text {eTES }}$ by the amount $P_{0} / G_{e-p}=0.154 \mathrm{mK}$.

We simulate the absorption of a gamma ray by perturbing the steady-state temperature of the absorber with an increase of $E / C_{A b s}$. The differential equations are solved numerically using a Runga-Kutta method with adaptive step sizes. The solutions give the temperature evolution of each component. Fig. 5.3 shows the temperature response of the microcalorimeter from absorption of a photon at $t=0$.

The four-element model is accurate in describing the detector. However, it also requires intensive calculations. With some good approximations, the detector can be modeled as a two element device without loss of accuracy.

The absorber and Stycast are strongly coupled together and can be considered as an effective absorber with heat capacity 


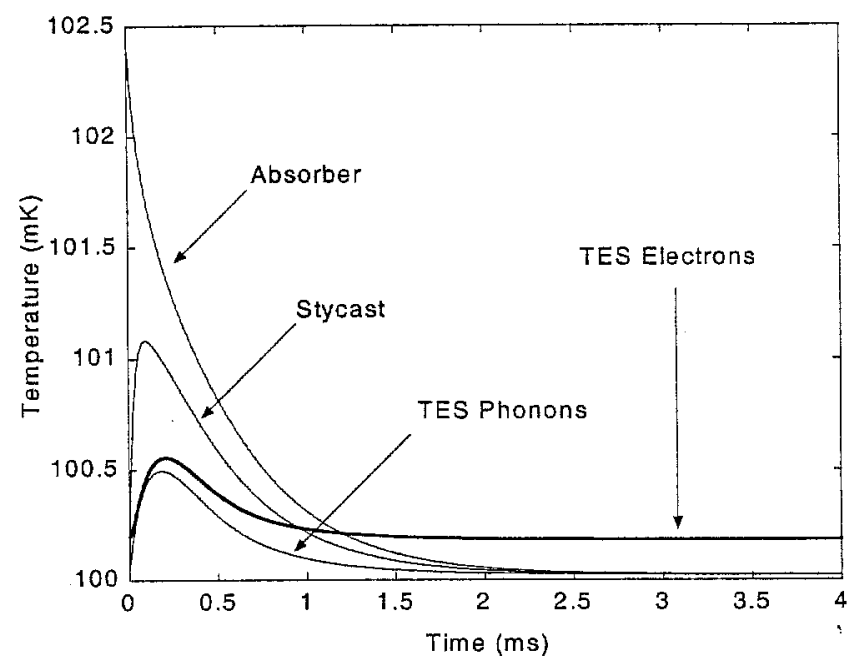

Fig. 5.3 Modeled temperature response profiles for 4-element model.

Eq. 5.4

$$
C_{A b s(e f f)}=C_{A b s}+C_{S t y}
$$

The couplings between the absorber and the TES include the thermal conductivity of the Stycast and two Kapitza boundary interfaces. For the two-element model, we can define an effective Stycast coupling given by

Eq. 5.5

$$
\frac{1}{G_{\text {Stycast (eff })}}=\frac{2}{G_{K}}+\frac{1}{G_{\text {Stycast }}}=\frac{2}{G_{K(e f f)}} .
$$

In the TES, the electron-phonon coupling is much stronger than $G_{S i N}$, keeping the phonon temperature and the electron temperature in the TES close together. The phonons and the electrons of the TES can be regarded as one element. But since the phonon heat capacity is three orders of magnitude smaller than the electron heat capacity, it can be neglected altogether. 


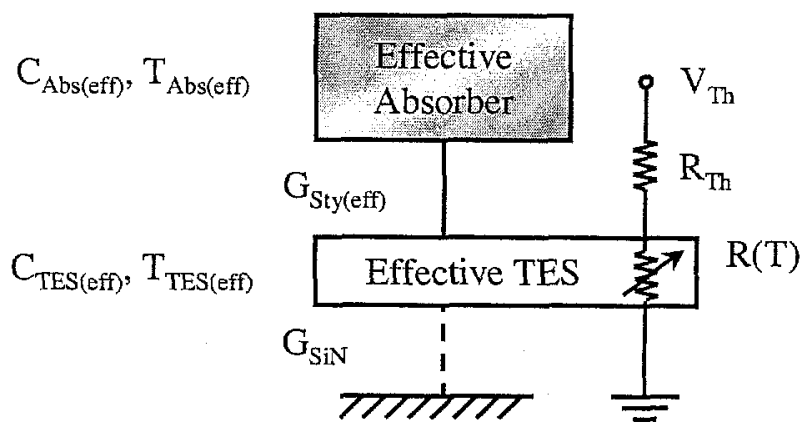

Fig. 5.4 Thermal diagram of the two-element approximation of the microcalorimeter.

The two relevant elements are then the effective absorber-Stycast combination and the TES electrons. Fig. 5.4 shows the components of the two-element approximation. The two-element approximation only requires a system of two differential equations,

Eq. 5.6

$$
\frac{d}{d t} T_{\text {Abs (eff) }}=-\frac{G_{\text {Strefft })}}{C_{A b s(e f f)}}\left(T_{A b s(e f f)}-T_{T E S(e f f)}\right)
$$

$$
\frac{d}{d t} T_{T E S(e f f)}=\frac{G_{S t y(e f f)}}{C_{T E S(e f f)}}\left(T_{A b s(e f f)}-T_{T E S(e f f)}\right)-\frac{K_{S i N}}{C_{T E S(e f f)}}\left(T_{T E S(e f f)}^{N}-T_{B a t h}^{N}\right)+\frac{V_{T h}^{2} R(T)}{C_{T E S(e f f)}\left(R_{T h}+R(T)\right)^{2}}
$$

Fig. 5.5 shows the detector response to a gamma ray in the two-element model compared with the absorber and TES electron solutions from the four-element model. The effective parameters used in the two-element model are calculated from the same default parameters used in the four-element model. In the two-element model, the steady-state temperature of both the effective absorber and the effective TES is exactly $T_{o p}$, whereas in the four-element model the steady-state absorber temperature is below $T_{o p}$. This difference does not affect the simulation, since the steady-state temperature of the TES is 
the same in both models. The signals from both models differ by $\sim 3 \%$ in pulse height and differ by $\sim 2 \%$ in decay time. The two-element approximation is good for $G_{e-p} \gg$ $G_{S i N}$. If $G_{e p}$ becomes comparable to $G_{S i N}$, then there will be significant phonon loss into the substrate and the two-element approximation loses validity.

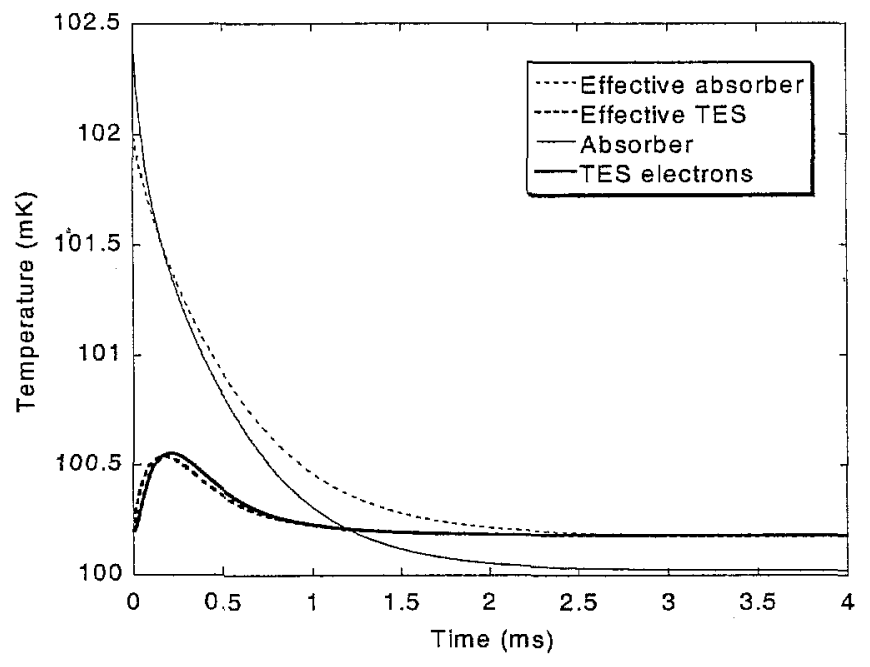

Fig. 5.5 Comparison of two-element and four-element models.

The numerical solutions give us the TES temperature profile. The corresponding current signal is

Eq. 5.7

$$
I=\frac{V_{T h}}{R_{T h}+R(T)} .
$$

Fig. 5.6 shows the current signal pulse calculated from the two-element model. As expected, the signal is a negative pulse originating from the equilibrium current. The signal amplitude is $I_{\text {Signal }}=8.61 \mu \mathrm{A}$. 


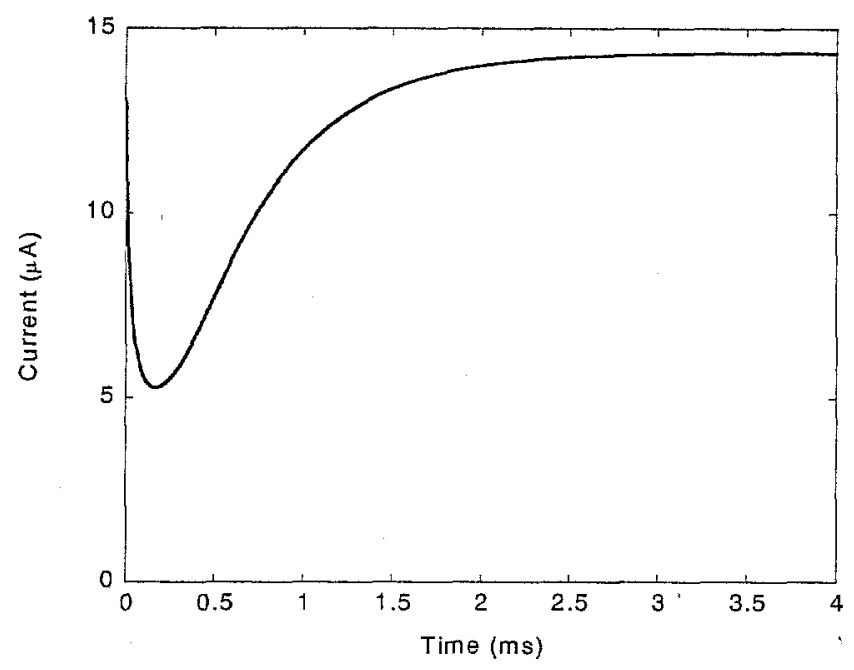

Fig. 5.6 Modeled current signal pulse.

\subsection{Numerical noise calculations}

The multi-component nature of our microcalorimeter sets a lower limit to the TES response time. From Eq. 3.32 we see that, under a voltage bias, the decay time of the signal can be shortened drastically for sufficiently large $\alpha$. However, in our real microcalorimeter, the decay time is ultimately restricted by how fast energy can flow out of the absorber through the Stycast bottleneck into the TES, $C_{A b s(e f f)} / G_{S t y(e f f)}$. The modeled signal has a decay time of $\tau=430 \mu \mathrm{s}$, which is close to the bottleneck decay time of $C_{\text {Abs(eff) }} / G_{S t y(e f f)}=419 \mu \mathrm{s}$. Therefore, we must use the noise expressions for slow energy arrival. It is interesting to note that the natural decay time for the microcalorimeter is $C_{\text {Total }} / G_{S i N} \approx 15 \mathrm{~ms}$. Electrothermal feedback was able to shorten the decay time of the system by over a factor of 30 before being limited by the bottleneck. 
We can include noise into the numerical model for comparison with noise predictions. We insert each noise power term separately into the differential equations and solve for the magnitude of the current response for that specific noise term,

Eq. 5.8

$$
\begin{aligned}
& \frac{d}{d t} T_{A b S(e f)}=-\frac{G_{S b y(e f f)}}{C_{A b S(e f f)}}\left(T_{A b s e f f)}-T_{T E S(e f f)}\right) \\
& \frac{d}{d t} T_{T E S(e f f)}=\frac{G_{S t r(e f f)}}{C_{T E S(e f f)}}\left(T_{A b s(e f)}-T_{T E S(e f f)}\right)-\frac{K_{S i N}}{C_{T E S(e f f)}}\left(T_{T E S(e f f)}^{N}-T_{B a t h}^{N}\right)+\frac{V_{T h}^{2} R(T)}{C_{T E S(e f f)}\left(R_{T h}+R(T)\right)^{2}}+\frac{P_{N}}{C_{T E S(e f f)}},
\end{aligned}
$$

where $P_{N}$ is a noise term. For phonon noise, we use Eq. 3.48, and for Johnson noise, we use Eq. 3.49. We substitute $\tau_{\text {Arr }}$ with $\tau$, the decay time of the modeled signal (not the decay time predicted by Eq. 3.32). We numerically solve the differential equations for the magnitude of temperature fluctuations due to the noise power sources. We write the noise current in a form analogous to Eq. 3.37 and using Eq. 5.7 for the current due to thermal fluctuations,

Eq. 5.9

$$
I_{P h}=\frac{V_{T h}}{R_{T h}+R\left(T_{P h}\right)}
$$

Eq. 5.10

$$
l_{J}=\frac{V_{J}}{R_{T h}+R_{0}}-\frac{V_{J}}{R_{T h}+R\left(T_{J}\right)}
$$

where $I_{P h}$ and $I_{J}$ are the phonon and Johnson noise currents, respectively, $T_{P h}$ and $T_{J}$ are the phonon and Johnson noise temperature fluctuation magnitudes. The magnitude of the 
numerically modeled phonon noise current is $I_{P h}=3.03 \mathrm{nA}$. The phonon noise current for slow energy arrival, using the modeled decay time as the bandwidth, is

Eq. 5.11

$$
\sqrt{2 k\left(T_{A b s}^{2}+T_{0}^{2}\right) G_{S t y} \tau}\left(\frac{I_{S i g n a l}}{E}\right)=2.23 n A,
$$

where the factor $I_{\text {Signal }} / E$ is the energy-to-current conversion. Similarly, the numerically modeled Johnson noise current is $I_{J}=0.396 \mathrm{nA}$, which is close to the slow energy arrival value of

Eq. 5.12

$$
\sqrt{\frac{4 k T_{0} R_{0}}{\tau}}\left(\frac{1}{R_{T h}+R_{0}}\right)=0.326 n A .
$$

We can calculate the slow energy arrival Johnson noise current for the bias resistor, again using the modeled decay time as the bandwidth,

Eq. 5.13

$$
I_{\text {Th }}=\sqrt{\frac{4 k T_{T h} R_{T h}}{\tau}}\left(\frac{1}{R_{T h}+R_{0}}\right)=0.46 n A
$$

The noise current for bath fluctuations of $10 \mu \mathrm{K}$ is given by Eq. 3.53,

Eq. 5.14

$$
I_{\text {Bath }}=\frac{1}{2} N T_{\text {Bath }}^{N-1} \frac{\Delta T_{\text {Bath }}}{T_{0}-T_{\text {Bath }}} I_{\text {Signal }}=0.572 n A
$$


The SQUID amplifier noise is

$\mathrm{Eq} .5 .15$

$$
I_{\text {SQUID }}=10 \frac{p A}{\sqrt{H z}} \frac{1}{\sqrt{2 \pi \tau}}=0.192 n A
$$

where the factor $2 \pi$ converts $\mathrm{Hz}$ into $\mathrm{rad} / \mathrm{sec}$. We can find the total noise by adding the noise currents in quadrature,

Eq. 5.16

$$
I_{\text {Total }}=\sqrt{I_{P h}^{2}+I_{J}^{2}+I_{T h}^{2}+I_{\text {Bath }}^{2}+I_{\text {SQUID }}^{2}} \approx 2.38 n A \text {. }
$$

For the signal shown in Fig. 5.6, the amplitude is $I_{\text {Signal }}=8.61 \mu \mathrm{A}$. Thus the signal to noise ratio is 3620 , giving us an energy resolution of $\Delta E \approx 39 \mathrm{eV}$ for a $60 \mathrm{keV}$ gamma ray by only considering frequencies up to $1 / \tau$.

\subsection{Optimization of microcalorimeter design}

With an appropriate numerical model and quantified noise sources, signal-to-noise performance of the detector can be mapped. Given the complex nature of the microcalorimeter, there are many parameters to consider. In general, the best signal-tonoise ratio is achieved with small heat capacities (increases signal size, reduces phonon noise), strong thermal couplings (faster pulses), high operating power, and high operating $\alpha$. Fabrication and operational limitations restrict the values of many parameters. Within these constraints, we find the optimum value that yields the best signal-to-noise ratio. 
A series of optimization calculations were performed by varying one parameter at a time. Of the remaining parameters, those that depend on the varying parameter also varied, and those that are independent were held constant. As usual, the microcalorimeter parameters and the default values are listed in Table 5.1.

As an example, consider the optimal $T_{c}$ of the TES, which is constrained by the opposing demands of heat capacity and operating power. A low $T_{c}$ corresponds to a low $T_{0}$ and smaller heat capacities, giving larger signals. However, for a fixed $T_{\text {Bath }}$, a high $T_{c}$ corresponds to a high operating power, also giving larger signals. The $T_{c}$ must therefore have an optimum value compromising on operating power and heat capacities. In the optimization of $T_{c}$, we vary the value of $T_{c}$ for each calculation. We also vary the dependent parameters of heat capacity, $T_{0}$, and $I_{0}$. Independent parameters such as $R_{0}$, $T_{\text {Bath }}$, etc. will remain constant.

\subsubsection{Vary operating points}

We begin by keeping the physical parameters constant and varying only the operating bias to probe the detector response. For a given microcalorimeter, the only two parameters that we can control are $T_{B a t h}$ and $V_{T h}$. While on the transition, changing $V_{T h}$ corresponds to changing $R_{0}$ and changing $T_{B a t h}$ changes $P_{B i a s}$. In essence, the only two parameters that we control are $R_{0}$ and $P_{\text {Bias }}$. The magnitude of the steady-state current may affect the transition width slightly, but we expect this effect to be small (see Section 7.4 , page 134$)$. 

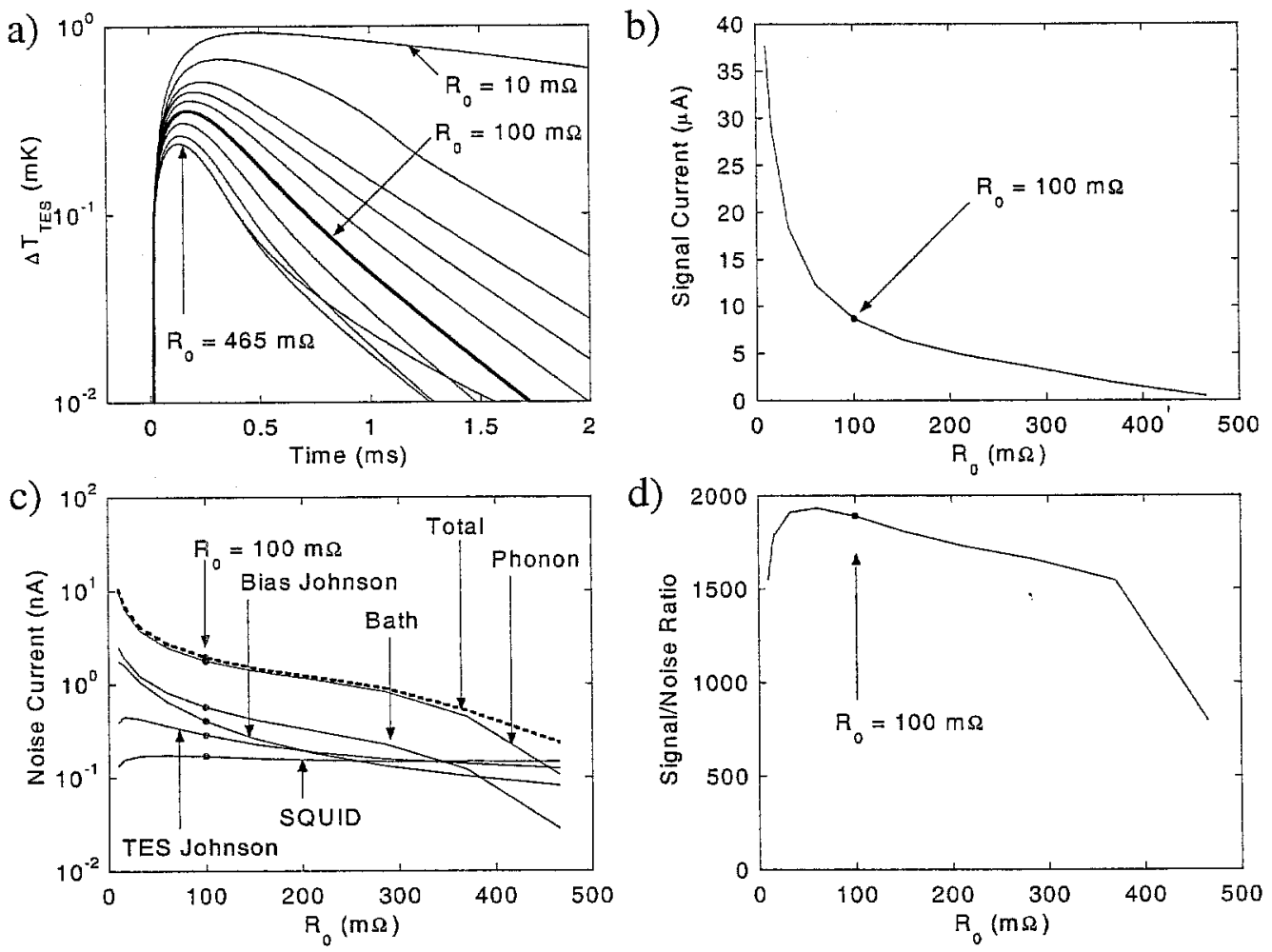

Fig. 5.7 Optimization of operating resistance.

\subsubsection{Operating resistance}

By changing $R_{0}$, we change $\alpha$ and $I_{0}$. At high $R_{0}, \alpha$ is small, giving small signals. At extremely low $R_{0}$, we may lose voltage bias (Section 3.3 , page 46 ) and lose negative electrothermal feedback. Furthermore, for extremely high $\alpha$, the spatial temperature distribution of the TES may become unstable (Section 4.2.2, page 75). Since our model includes the Thévenin bias resistance $R_{T h}$, we can study the effects of varying $R_{0}$. We model the microcalorimeter with a varying $R_{0}$ from $10 \mathrm{~m} \Omega$ to $465 \mathrm{~m} \Omega$. The temperature profiles are shown in Fig. 5.7a. We see that decay times are long for high $R_{0}$ due to low $\alpha$, but decay times are also long for low $R_{0}$, due to mixed voltage/current bias. Fig. 5.7b 
shows the current signal magnitude as a function of $R_{0}$. We see that for low $R_{0}$, the signal is large due to large $I_{0}$, and for high $R_{0}$ the signal is small due to small $\alpha$. From the decay time of the pulses, we can determine the bandwidth of the signal and quantify the noise sources. Fig. 5.7c shows the calculated noise currents for varying $R_{0}$. We see that phonon noise dominate in the region $R_{0}<450 \mathrm{~m} \Omega$, above which SQUD noise dominates. The corresponding signal-to-noise ratio is shown Fig. 5.7d. We see that the best energy resolution is found near $R_{0}=50 \mathrm{~m} \Omega$.

\subsubsection{Bath temperature}

For a given $T_{c}$, we can vary the bias power only by changing the bath temperature. However, as $T_{B a t h}$ decreases, so does the thermal conductivity of the silicon nitride membrane $G_{S i N}$, limiting the magnitude of the bias power. Similarly, we perform optimization calculations for $T_{\text {Bath }}$. Fig. 5.8a shows the temperature profiles for varying $T_{\text {Bath }}$ from $45 \mathrm{mK}$ to $95 \mathrm{mK}$ with $T_{c}$ fixed at $100 \mathrm{mK}$. We see that decay times shorten as $T_{\text {Bath }}$ decreases. Fig. $5.8 \mathrm{~b}$ shows the current signal magnitude.

We see that the signal size increases as $T_{\text {Bath }}$ decreases. It is interesting to note that the signal size no longer increases significantly at temperatures below $\sim 80 \mathrm{mK}$, but the signal-to-noise ratio continues to improve until $\sim 60 \mathrm{mK}$ when bath temperature fluctuations become negligible. 
a)
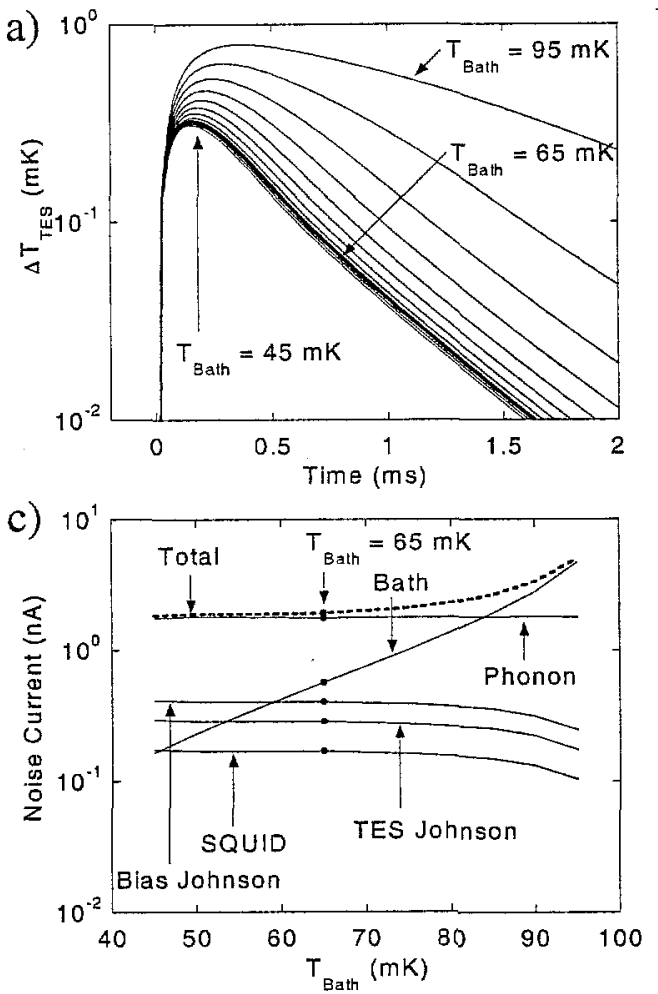

b)

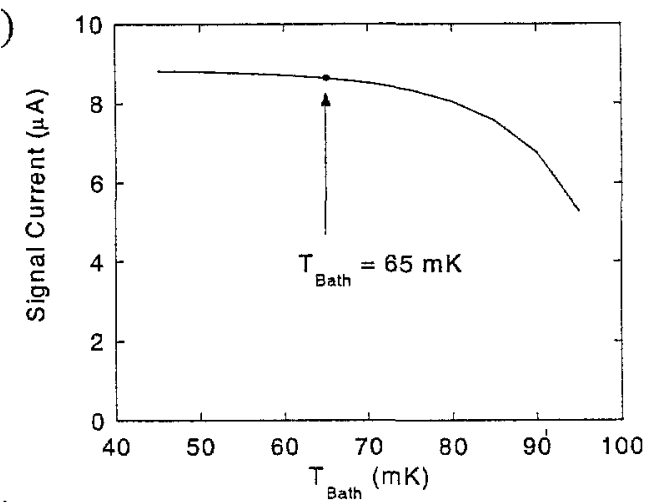

d)

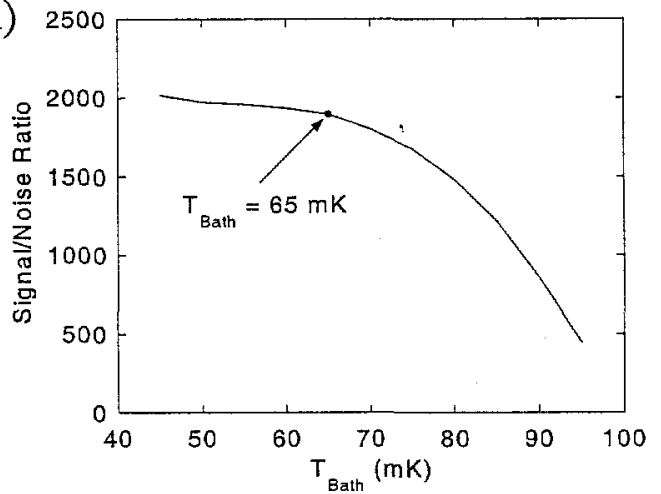

Fig. 5.8 Optimization of bath temperature.

As before, phonon noise dominate in the region $T_{\text {Bath }}<85 \mathrm{mK}$. Above $85 \mathrm{mK}$, the current noise due to bath fluctuations dominate. Interestingly, if the bath temperature fluctuations were much smaller, the signal-to-noise ratio would have a "flatter" overall response. This analysis tells us that the best performance is achieved at the lowest $T_{\text {Bath }}$. In practice, though, $T_{B a t h}$ is limited by the performance of the ADR to approximately 65 $\mathrm{mK}$. 


\subsubsection{Vary physical properties}

\subsubsection{Absorber volume}

In addition to varying operating bias points, we can also vary physical properties of the TES and investigate the detector response. For good gamma ray absorption efficiency, we want a large absorber. The size of the absorber contributes to the heat capacity, affecting the signal size and decay time. We modeled the microcalorimeter with a varying absorber volume from $0.125 \mathrm{~mm}^{3}$ to $1.25 \mathrm{~mm}^{3}$. Fig. $5.9 \mathrm{a}$ shows the TES temperature profiles from the absorption of a $60 \mathrm{keV}$ gamma ray for varying absorber volumes. We see that small absorber volumes have larger signals and shorter decay times, and vice versa, as expected. Fig. $5.9 \mathrm{~b}$ shows the current signal amplitude as a function of absorber volume. Fig. 5.9c shows the calculated noise currents for varying absorber volume. Fig. 5.9d shows the corresponding signal-to-noise ratios. We see that despite a factor of 10 variation in the absorber volume, the signal-to-noise ratio only varies by a factor of two. This is due to the fact that the dominant noise source in our modeling is phonon noise, which is independent of the absorber volume for a compound calorimeter in the slow energy arrival limit. In this comparison, we have fixed the thermal coupling between the absorber and the TES. In a real device, larger absorbers may require more structural support and possibly a larger thermal contact, resulting in more noise. From this analysis, we see that our default absorber volume of $0.250 \mathrm{~mm}^{3}$ is nearly optimal since the smaller absorber volume would drastically reduce the collection area in order to maintain a good absorption efficiency. We also see that we can increase the volume to over $1 \mathrm{~mm}^{3}$ and still maintain reasonably good resolving power as long as thermalization of the $\mathrm{Sn}$ is not a problem. 

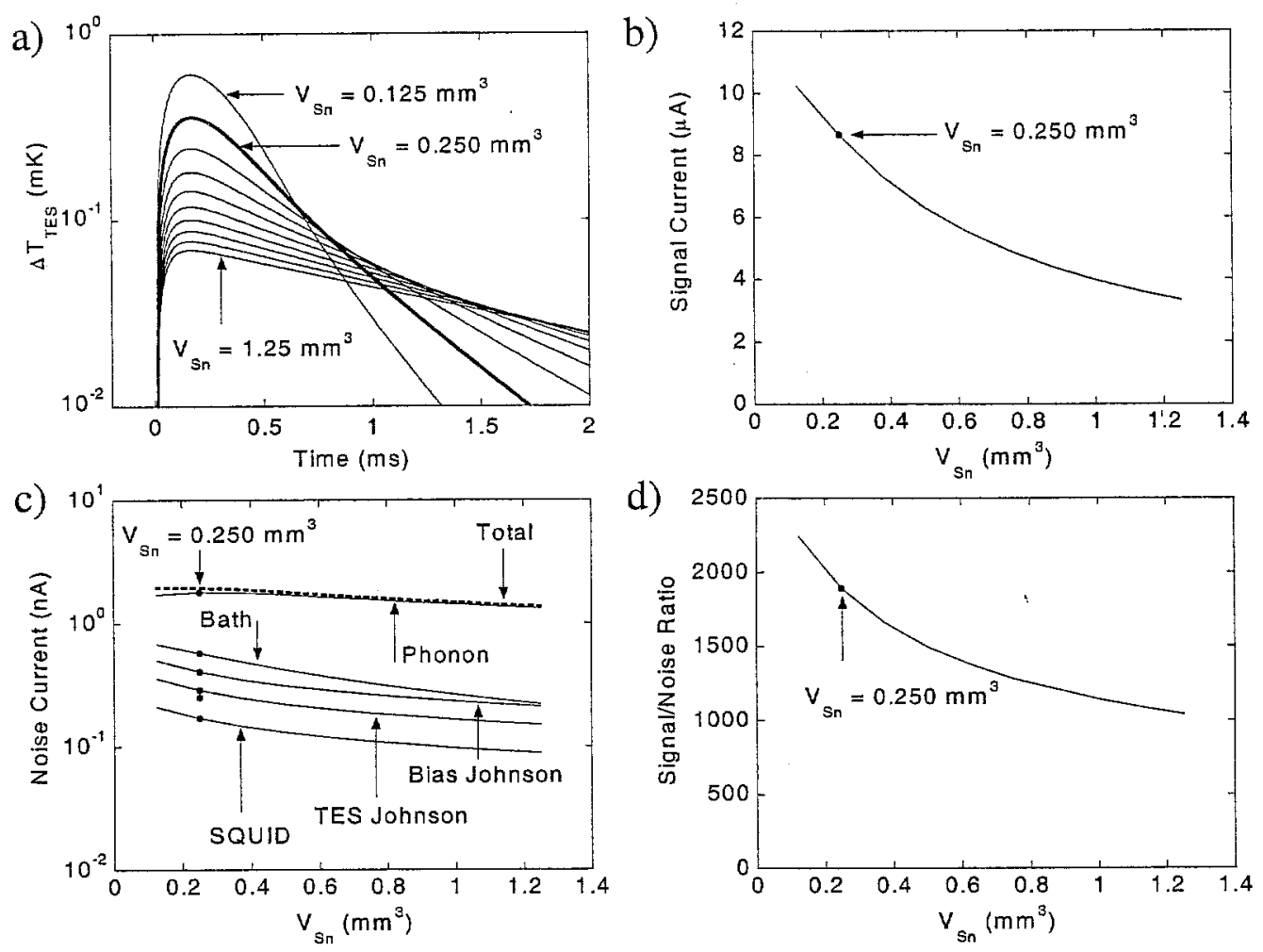

Fig. 5.9 Optimization of absorber volume.

\subsubsection{Diameter of Stycast epoxy}

Similarly, we perform optimization calculations for the dimensions of the Stycast epoxy.

Thicker Stycast increases heat capacity and reduces the thermal coupling between the absorber and the TES, so we expect the signal-to-noise performance to degrade. We therefore fix the thickness at $25 \mu \mathrm{m}$, which is as thin as we have been able to make it. Larger diameter of Stycast increases both thermal conductivity and heat capacity, which have opposing effects on the signal size. We know that larger heat capacity decreases signal size. Larger Stycast thermal conductivity increases signal size by allowing the absorber and TES to equilibrate quickly before any significant amount of energy 
dissipates into the cold bath. We modeled the microcalorimeter with a varying diameter of Stycast from $50 \mu \mathrm{m}$ to $550 \mu \mathrm{m}$. Fig. 5.10a shows the temperature pulses.

We see that the signal is maximized for $D_{\text {Stycast }} \sim 350 \mu \mathrm{m}$ (Fig. $5.10 \mathrm{~b}$ ). Again, we see that phonon noise dominates. The signal-to-noise ratio is optimum at $D_{\text {Stycast }}=100 \mu \mathrm{m}$ (Fig. 5.10d). The large variation of signal-to-noise ratio suggests that the positive effects of increased bandwidth by increasing the thermal conductivity of the Stycast are negligible to the increased phonon noise due (Fig. 5.10c).
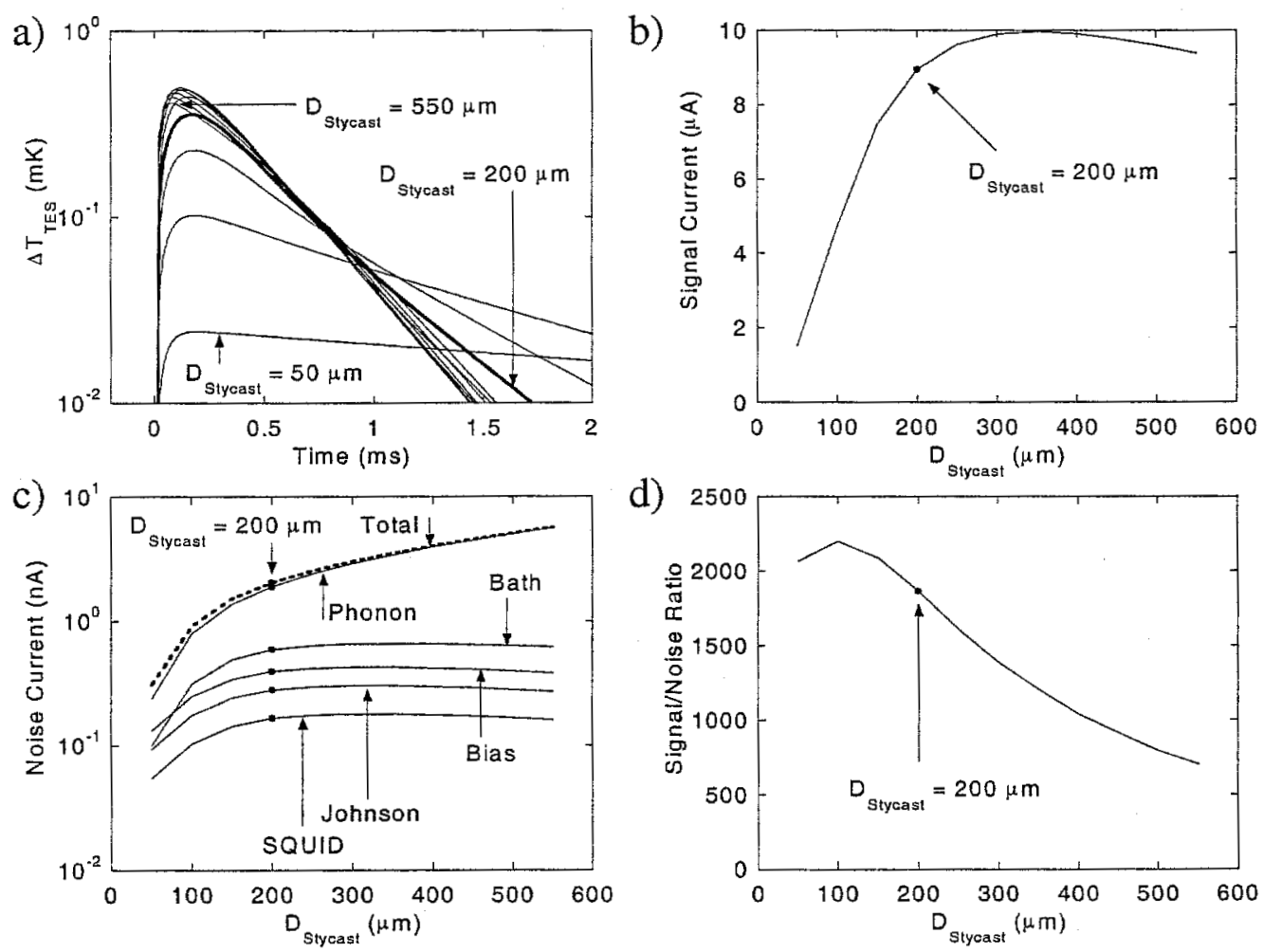

Fig. 5.10 Optimization of Stycast diameter. 


\subsubsection{Critical temperature of the TES}

As described earlier, the optimal $T_{c}$ is constrained by heat capacity and operating power. We varied $T_{c}$ from $80 \mathrm{mK}$ to $180 \mathrm{mK}$ with a fixed $T_{\text {Bath }}$ and modeled the microcalorimeter response. Fig. 5.11a shows the temperature response to $60 \mathrm{keV}$ gamma rays. The TES change in temperature is greatest for low $T_{c}$ due to low heat capacity. The signal size is dependent on the bias current as well as the change in temperature, as described by Eq. 3.24. We see in Fig. 5.11b that the signal size is largest for $T_{c} \sim 110$ mK. Fig. 5.11c shows that at $T_{c}<85 \mathrm{mK}$, phonon noise no longer dominates as current noise due to bath fluctuations become increasingly more important as expected. The signal-to-noise ratio is greatest for $T_{c} \sim 90 \mathrm{mK}$.
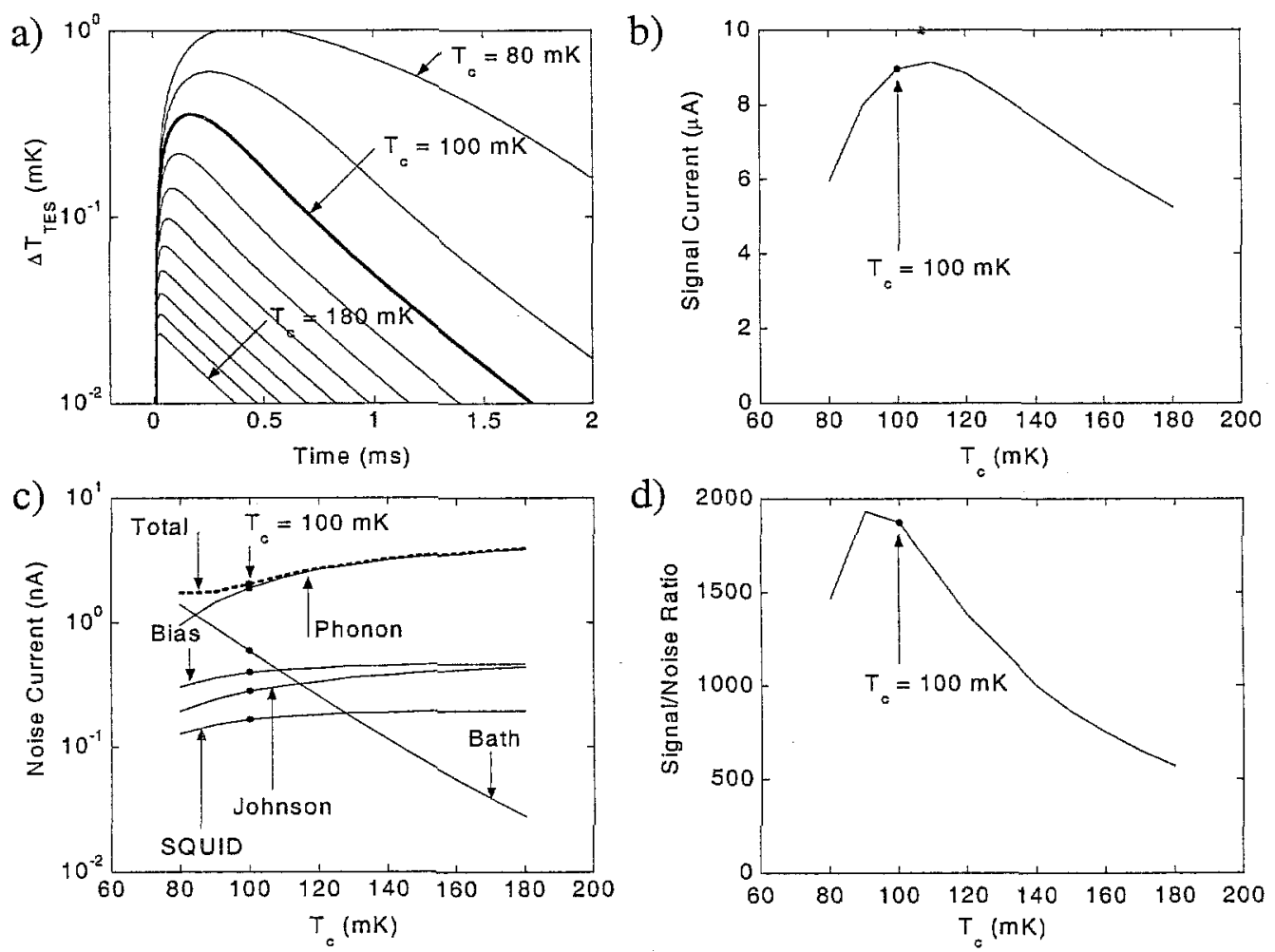

Fig. 5.11 Optimization of superconducting transition temperature. 


\subsubsection{Transition width of the TES}

In Section 3.3 (page 46) we stated that the sensitivity of the microcalorimeter is dependent on the steepness of the transition. We modeled the detector response for varying superconducting transition widths from $0.25 \mathrm{mK}$ to $2.75 \mathrm{mK}$, while maintaining a constant $R_{0}$ and $T_{0}$. As before, the temperature profiles are shown in Fig. 5.12a, the signal amplitudes in Fig. 5.12b, the noise contributions in Fig. 5.12c, and the signal-tonoise ratio in Fig. $5.12 \mathrm{~d}$. We see that increasing $\Delta T_{c}$ by a factor of ten only decreases the signal-to-noise ratio by $\sim 20 \%$. The dominant noise source is, again, phonon noise, which is independent of the transition width.
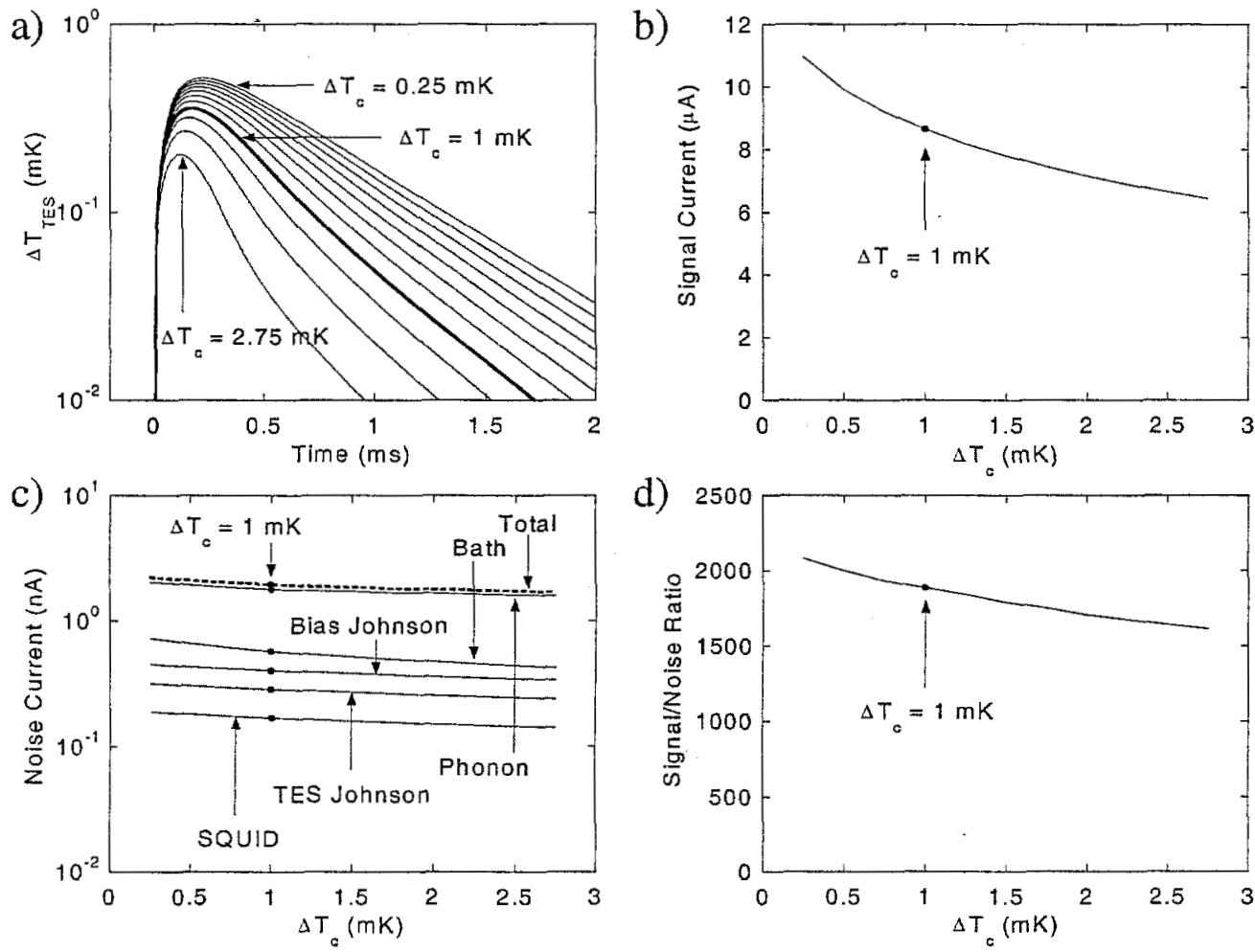

Fig. 5.12 Optimization of superconducting transition width. 
Note that Irwin [2] and Lindeman [3] relates energy resolution to $\alpha$,

Eq. 5.17

$$
\Delta E \sim \frac{1}{\sqrt{\alpha}} .
$$

This is only true if we only consider the simple one-element phonon noise and TES Johnson noise. Our optimization includes noise contributions from nearly all quantifiable noise sources.

These optimization routines allow us to develop intuition on the relative importance of various physical properties. From the preceding calculations, we see that many parameters affect the signal size, but it is $T_{c}$ and $T_{B a t h}$ that has the largest influence in signal size and signal-to-noise ratios. Fig. 5.13 shows the optimization of $T_{c}$ for various $T_{B a t h}$. For $T_{c}$ above $100 \mathrm{mK}$, the signal size is nearly independent of $T_{B a t h}$ for the range of $T_{B a t h}$ that we examined. However, the signal-to-noise ratio can degrade significantly with increasing $T_{B a t h}$ for $T_{c}<110 \mathrm{mK}$. We also notice that for higher $T_{B a t h}$, the optimum $T_{c}$ shifts to higher temperature.

In the fabrication of the microcalorimeter, we expect the Stycast dimensions to have low repeatability. We perform similar optimizations for Stycast diameter for different Stycast thicknesses, shown in Fig. 5.14. We see that despite a significant variation in signal size due to varying Stycast thickness, the signal-to-noise ratio is relatively unaffected. 
We presented a numerical model that describes the performance of the microcalorimeter.

We can use this model as a tool to optimize the design of the microcalorimeter. This model also allows us to predict the behavior of the microcalorimeter under different circumstances. Furthermore, we can use this model to infer parameters of the microcalorimeter that are otherwise difficult or impossible to measure.
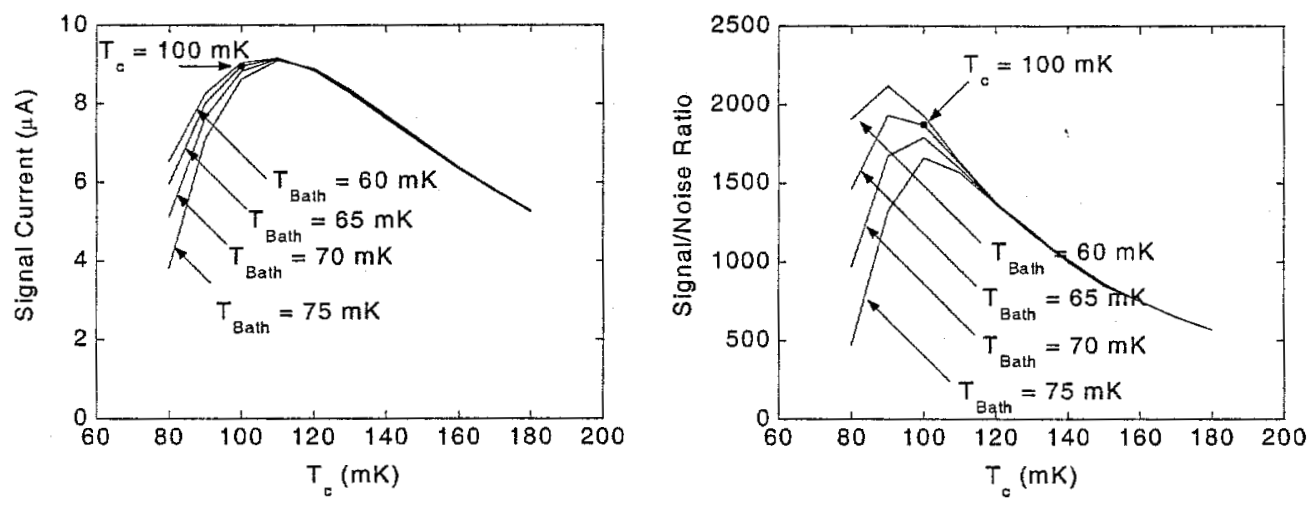

Fig. 5.13 Optimization of transition temperature for different bath temperatures.
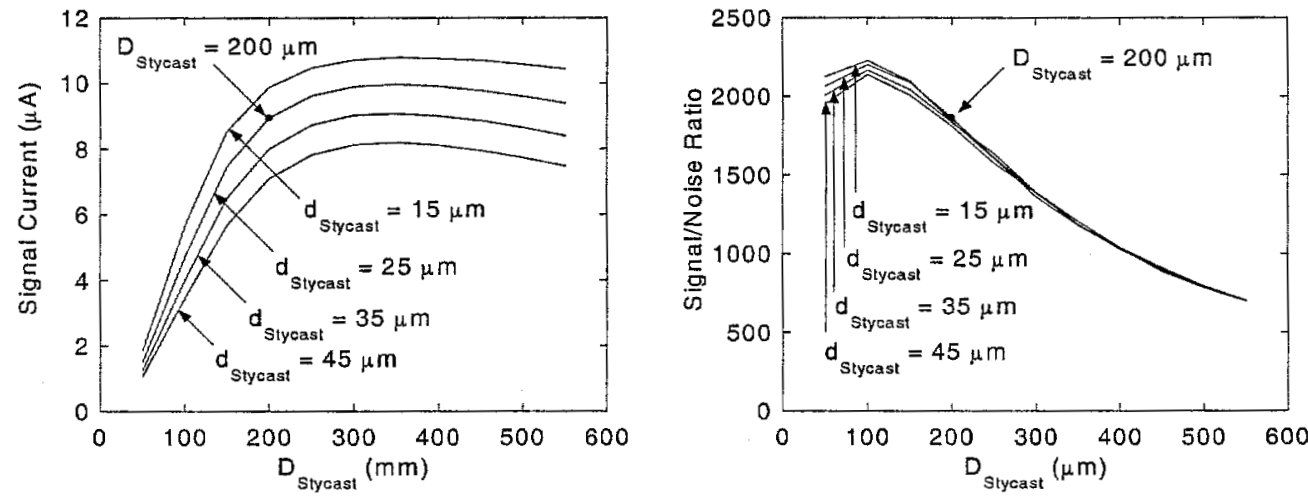

Fig. 5.14 Optimization of Stycast diameter for difference Stycast thicknesses. 


\section{References}

[1] F. Pröbst, M. Frank, S. Cooper, P. Colling, D. Dummer, P. Ferger, G. Forster, A. Nuciotti, W. Seidel, L. Stodolsky, Journ. Low Temp. Phys., 100(1/2), 65 (1995).

[2] K.D. Irwin, G.C. Hilton, D.A. Wollman, et al., Journ. Appl. Physics, 83(8), 3978 (1998).

[3] M.A. Lindeman, Ph.D. Dissertation, University of California, Davis (2000). 
Chapter 6

\section{FABRICATION AND TESTING}

\subsection{Fabrication of devices}

Fabrication of our TES microcalorimeters is done completely at LLNL using the facilities of the Microtechnology Center, the Material Science and Technology Division, and the Advanced Detector Group (ADG). The TES is a thin-film device made by standard semiconductor industry fabrication techniques. The fabrication procedure of our microcalorimeter are outlined in Fig. 6.1. We briefly discuss each process step. Procedure run sheets are provided in Appendix A.

\subsubsection{SiN membrane windows}

The SiN membranes are fabricated at the Microtechnology Center. We purchased standard 4"-diameter Si wafers with orientation $(1,0,0)$ from industrial vendors. Typical wafer thickness is $\sim 0.5 \mathrm{~mm}$ (Fig. 6.1a). The doping and resistivity of the wafers is irrelevant to the microcalorimeter, since the Si wafers only serve as a substrate and a cold bath. A $\sim 0.5 \mu \mathrm{m}$ thick layer of SiN is thermally grown onto both sides of the wafers (Fig. 6.1b). 
a. Silicon substrate.

b. Silicon nitride (SiN) thermally grown on $\mathrm{Si}$
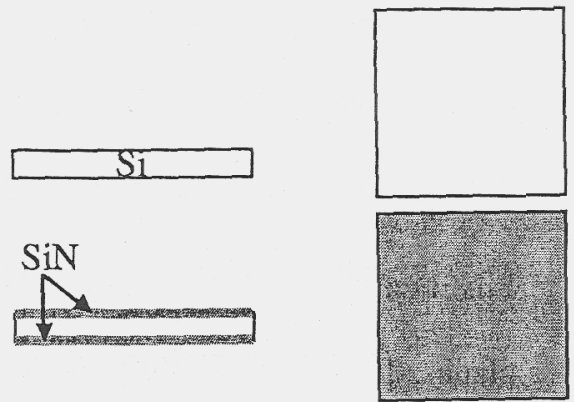

c. Hole created in SiN by reactive ion etching.
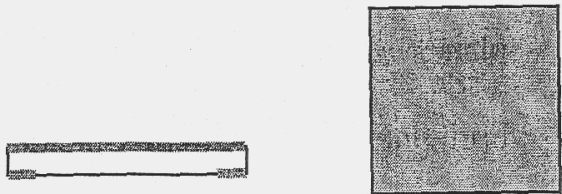

d. Si etched away to create membrane window.

e. TES deposited by dc sputtering and defined by photolithographic lift-off.
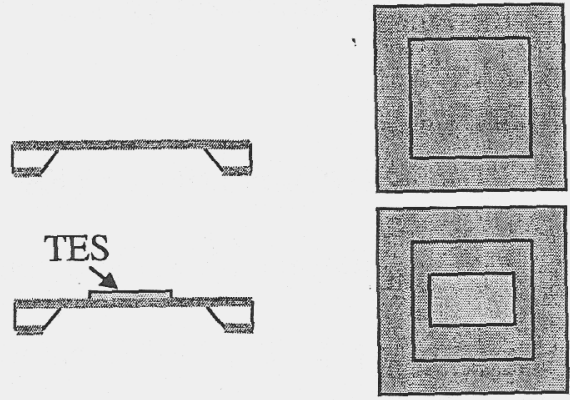

f. Al leads deposited by dc sputtering and defined by photolithographic lift-off.

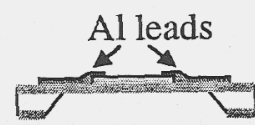

g. Epoxy applied and spacers positioned.
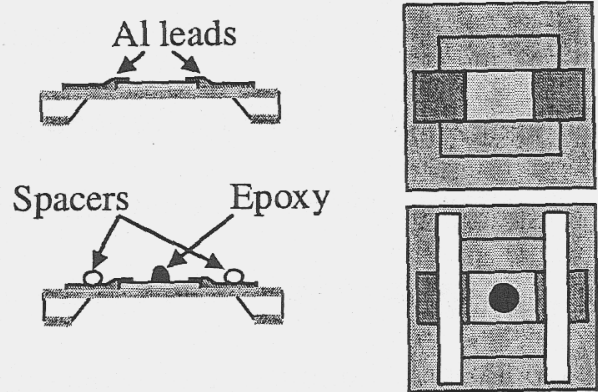

h. Absorber coupled to epoxy while suspended by spacers.
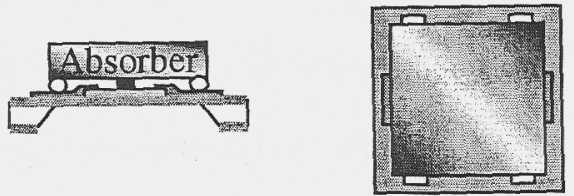

i. Spacers removed after epoxy cures, leaving free-standing absorber.
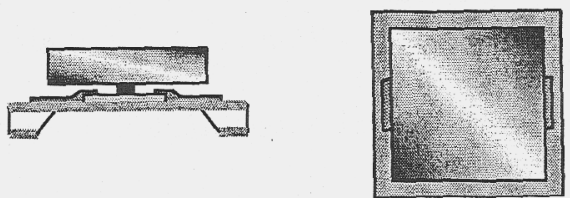

Fig. 6.1 Illustration of fabrication steps with cross sectional and top views. 
On the back side of each wafer, an array of $2 \mathrm{~mm} \times 2 \mathrm{~mm}$ square holes is made in the SiN by $\mathrm{CF}_{4}$-based reactive ion etching (Fig. 6.1c). The exposed $\mathrm{Si}$ is wet etched completely through the thickness of the wafer with a $\mathrm{KOH}$ solution at $\sim 60^{\circ} \mathrm{C}$, leaving a suspended SiN membrane (Fig. 6.1d). The etchant preferentially removes $\mathrm{Si}$ along the $(1,1,1)$ planes, leaving a prismoidal cavity with the SiN membrane as a base and the $(1,1,1)$ planes as the trapezoidal walls. The $(1,1,1)$ planes are at an angle of $54.74^{\circ}$ from horizontal, thus, each SiN membrane has an area of $\sim 1.3 \mathrm{~mm} \times \sim 1.3 \mathrm{~mm}$. The wafers are then diced into $1 " \times 0.25$ " strips, each with three membrane windows.

\subsubsection{Mo/Cu multilayer TES}

The multilayer superlattices are fabricated in the Material Science and Technology Division [1]. The $\mathrm{Mo} / \mathrm{Cu}$ multilayer structure is deposited by dc magnetron sputtering (Fig. 6.1e). Typical base pressure is $\sim 10^{-7}$ Torr. Partial pressure of Ar gas during sputtering is $2 \mathrm{~m}$ Torr. Sputtering power is typically $150-250 \mathrm{~W}$ for both metals. The sputtering system is cylindrical, with the substrates mounted on a rotating platform. The sputter guns are mounted above the platform on opposite sides of the chamber with baffles to minimize cross-contamination. The platform is rotated at a rate of several seconds per cycle. For each complete rotation, the substrate is coated with one layer of each metal. The thickness of each metal is determined by their respective sputter powers. The rms variation of the thickness of the metal is $<1 \AA$. The number of layers is determined by the total number of rotation cycles. Fig. 6.2 shows a TEM micrograph of a sample $\mathrm{Mo} / \mathrm{Cu}$ multilayer superlattice. The pattern of the $\mathrm{Mo} / \mathrm{Cu}$ multilayer film is defined by photolithographic lift-off. Typical photoresist thickness is $\sim 1.8 \mu \mathrm{m}$. 


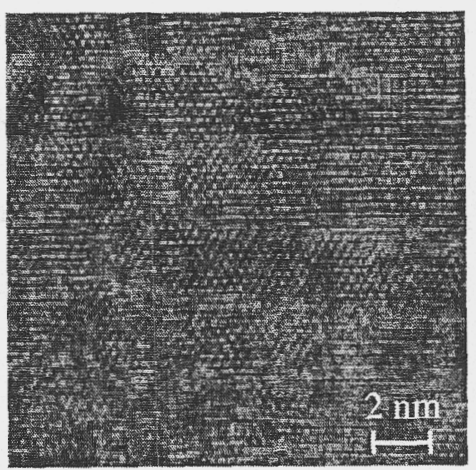

a)

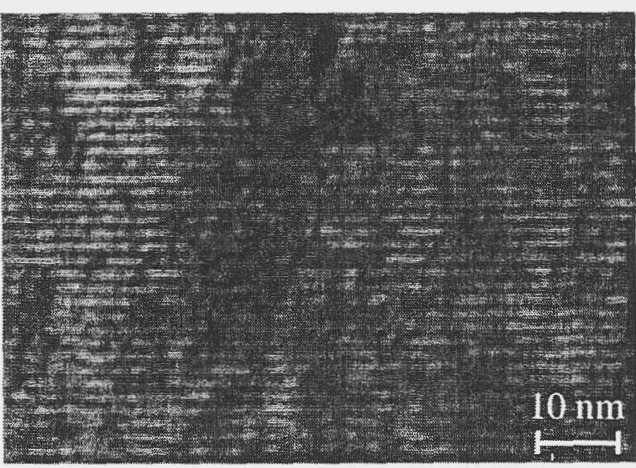

b)

Fig. 6.2 TEM micrographs showing typical $\mathrm{Mo} / \mathrm{Cu}$ multilayer structures.

a) High magnification image. The layers are seen as horizontal bands. Higher contrast bands are Mo, lower contrast bands are Cu. Granular structure are individual atoms. b) Lower magnification image showing many alternating layers of $\mathrm{Mo}$ and $\mathrm{Cu}$.

\subsubsection{Superconducting Al electrical leads}

The superconducting Al bias leads are fabricated in the Advanced Detector Group's UHV thin film deposition laboratory (Fig. 6.1f). Prior to deposition, native oxide on the surface of the $\mathrm{Mo} / \mathrm{Cu}$ multilayer is removed by ion milling to ensure good ohmic contact with the Al leads. Partial pressure of Ar for ion milling is 0.5 mTorr. Ions are accelerated through 500 volts at a rate of $20 \mathrm{~mA}$. The films are deposited by dc magnetron sputtering. Typical base pressure is $\sim 10^{-8}$ Torr. Partial pressure of Ar gas during sputtering is $3 \mathrm{mTorr}$. Sputtering power is typically $100 \mathrm{~W}$. Film pattern is defined by photolithographic lift-off.

\subsubsection{Stycast 2850FT epoxy}

Stycast 2850FT is a glass-filled, two-part epoxy [2]. The glass beads in the Stycast have an average diameter of $\sim 6 \mu \mathrm{m}$. The high viscosity of the Stycast makes it unsuitable for most picoliter-scale fluid dispensing systems. After mixing, the epoxy is deposited on 
the $\mathrm{Mo} / \mathrm{Cu}$ TES film manually (Fig. 6.1g) with a wire loop made with $25 \mu \mathrm{m}$ diameter, $99 \% \mathrm{Al}, 1 \%$ Si bonding wire. The diameter of the loop itself is $\sim 100 \mu \mathrm{m}$. A volume of $\sim 10 \mathrm{pL}$ can be reproduced with a error of $\pm 50 \%$. The Stycast can be formed into a cylindrical shape by its own surface tension and placement of spacers to suspend the absorber while the epoxy cures. The spacers are also fashioned from $25 \mu \mathrm{m}$ diameter bonding wires.

\subsubsection{Sn absorber}

We purchased $99.99 \%$ high-purity, polycrystalline, $0.250 \mathrm{~mm}$ thick, bulk $\mathrm{Sn}$ foil from Goodfellow Corporation [3]. The foils were cut into $1 \mathrm{~mm} \times 1 \mathrm{~mm}$ squares with a sharp blade. The absorbers are manipulated with fine-tip vacuum tweezers and placed on top of the epoxy and suspended by the spacers (Fig. 6.1h). The epoxy is allowed to cure for at least 24 hours at room temperature, after which the spacers were carefully removed. The completed microcalorimeter is shown in Fig. 6.1i. The collection area of the absorber is larger than the TES underneath it, eliminating direct absorption events of low energy photons in the TES film. Fig. 6.3 shows an optical micrograph of the Sn absorber supported by cylindrical Stycast.

This fabrication process is relatively crude by semiconductor standards. However, the merits of the device are determined by the superconducting transition and the thermal couplings to the absorber and cold bath. The steepness and linearity of the superconducting transition may not necessarily improve with a full-wafer, wet etch process (Section 1.4.3). 


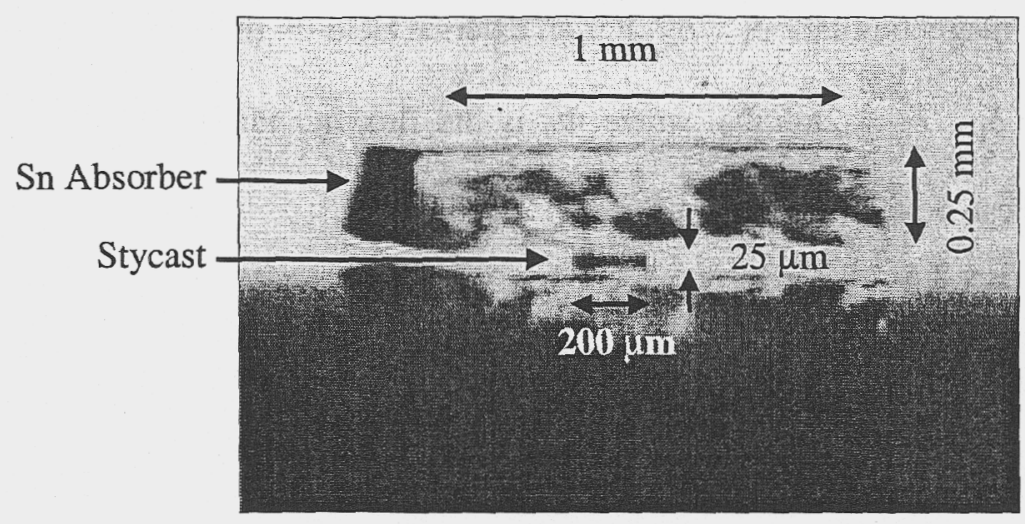

Fig. 6.3 Optical micrograph showing the absorber supported by Stycast epoxy.

The epoxy is a critical component of the microcalorimeter. However, microfluidic dispensing systems are generally unable to deposit Stycast due to its high viscosity. Other means of adhering the absorber to the TES film are currently being investigated (Section 1.4.3). Despite its lack of superficial cosmetic appeal, our procedure fabricates a fully functional microcalorimeter capable of high resolution gamma-ray spectrometry.

\subsection{Testing of devices}

Testing of our TES microcalorimeters are performed using the facilities of the ADG. All cryogenic systems and bias electronics were built by the ADG. All instrument interfacing and read-out software were also written by the ADG.

\subsubsection{SQUIDs}

The current signal of our microcalorimeter is read-out by a dc SQUID (superconducting quantum interference device) array current amplifier. A dc SQUID consists of a superconducting ring interrupted by two Josephson junctions (Fig. 6.4a). The phase differences across the junctions are denoted by $\delta_{1}$ and $\delta_{2}$, respectively. In the absence of 
an external magnetic field, $\delta_{1}=\delta_{2}$. For an external field $\Phi$, the phase differences around the ring is $\delta_{1}-\delta_{2}=2 \pi \Phi / \Phi_{0}$, where $\Phi_{0}$ is the flux quantum (Eq. 3.17). The phase differences can be rewritten as

Eq. 6.1

$$
\delta_{1}=\delta_{0}+2 \pi \frac{\Phi}{\Phi_{0}}, \delta_{2}=\delta_{0}-2 \pi \frac{\Phi}{\Phi_{0}}
$$

where $\delta_{0}$ is a constant. From the dc Josephson relation (Eq. 3.18), we write the total Josephson current as the sum of the currents through each junction

Eq. 6.2 $J_{\text {Total }}=J_{0}\left(\sin \left(\delta_{0}+2 \pi \frac{\Phi}{\Phi_{0}}\right)+\sin \left(\delta_{0}-2 \pi \frac{\Phi}{\Phi_{0}}\right)\right) \delta_{1}=2 J_{0} \sin \delta_{0} \cos \left(2 \pi \frac{\Phi}{\Phi_{0}}\right)$

We see that the phase difference across each junction interferes macroscopically and produces an oscillating total current with a periodicity of $\Phi_{0}$. With an appropriate resistance, we measure the Josephson current as a voltage. The dc SQUID is in essence a flux-to-voltage converter. Fig. 6.4b shows the voltage-flux $(V-\Phi)$ characteristic of a dc SQUID. For more details on de SQUIDs, please refer to Turner and Van Duzer [4].

If we want to measure the current in a circuit, we insert an input coil into the circuit. Current passing through the coil will generate a magnetic field that can be sensed by the SQUID and measured as change in the SQUID voltage. The steepness of the slope of the V- $\Phi$ response is indicative of the sensitivity of the SQUID. 


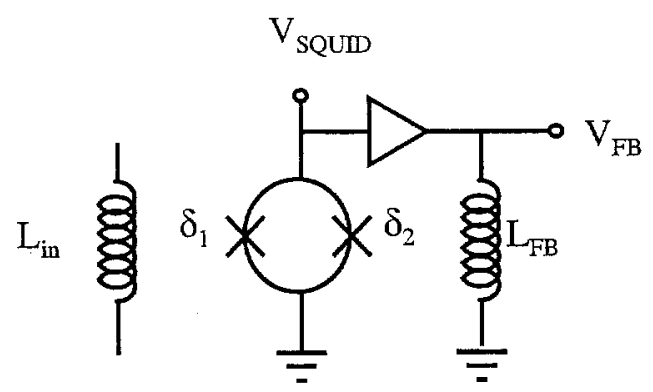

a)

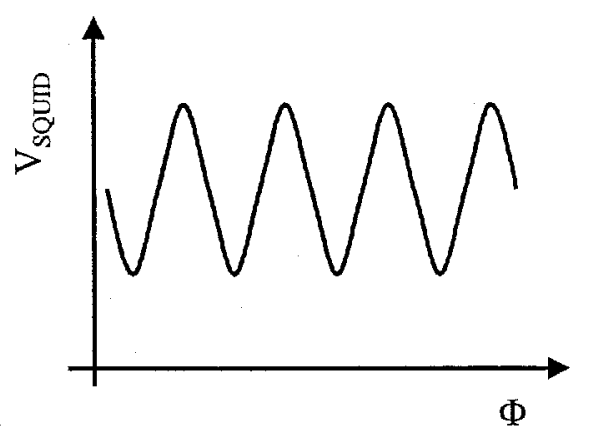

b)

Fig. 6.4 a) De SQUID circuit with input and feedback coils. b) De SQUID current vs. applied field.

However, the V- $\Phi$ response of the SQUID is linear over a very small range. To remedy this, we can operate the SQUID in a flux-locked feedback mode. In this mode, a feedback coil applies an opposing field proportional to the signal such that the total flux in the SQUID remains constant. With the SQUTD held at a constant flux, the response becomes impervious to any nonlinearities in the V- $\Phi$ characteristic. The response of the SQUID is then linear, with the signal proportional to the current flowing through the feedback coil.

Our dc SQUIDs are purchased from Hypress, Inc. [5]. These particular SQUTDs are series arrays with 208 SQUIDS. The arraying of SQUIDs in series serves as gain to the V- $\Phi$ response and better impedance matching with external FET electronics.

\subsubsection{Circuit}

The TES requires a strong voltage bias at the operating resistance of $\sim 0.1 \Omega$. Most commercially available voltage supplies have relatively large output impedances and are 
unable to provide strong voltage bias for such a small load resistance. Furthermore, the two-wire lead resistance from room temperature to the cold stage is much greater than $R_{0}$, making it impossible to apply a voltage bias directly. Instead, we use a low-impedance voltage divider near the cold stage. Fig. 6.5 shows the TES bias and SQUID read-out circuits. Bias to the TES is applied at $V_{\text {Bias }}$ through a voltage divider consisting of the bias resistor $R_{\text {Bias }}$ and the shunt resistor $R_{\text {Shunt }}$. The TES (denoted as a variable resistor) is in parallel with $R_{\text {Shunt }}$. For $R_{\text {Shunt }}<0.1 R_{0}$, the TES is under strong voltage bias. The current flowing through the TES is measured with a SQUID with an input inductance $L=$ $0.25 \mu \mathrm{H}$ in series with the TES. The input inductor is shunted with $R_{L}=20 \Omega$. The SQUID is operated in the flux-locked feedback mode such that the feedback voltage $V_{F B}$ is proportional to the current of the TES.

For frequencies less than $R_{0} / L$ where the inductor has negligible impedance, the current through the TES is given by

Eq. 6.3

$$
I_{T E S}=\frac{1}{B} \frac{V_{F B}}{R_{F B}}
$$

where $B$ is the ratio of magnetic flux sensed by the SQUID from each inductor. For our SQUDD, typical values of $B$ range from 8 to 15 , depending on the geometry of the particular SQUID. The voltage across the TES is given by 


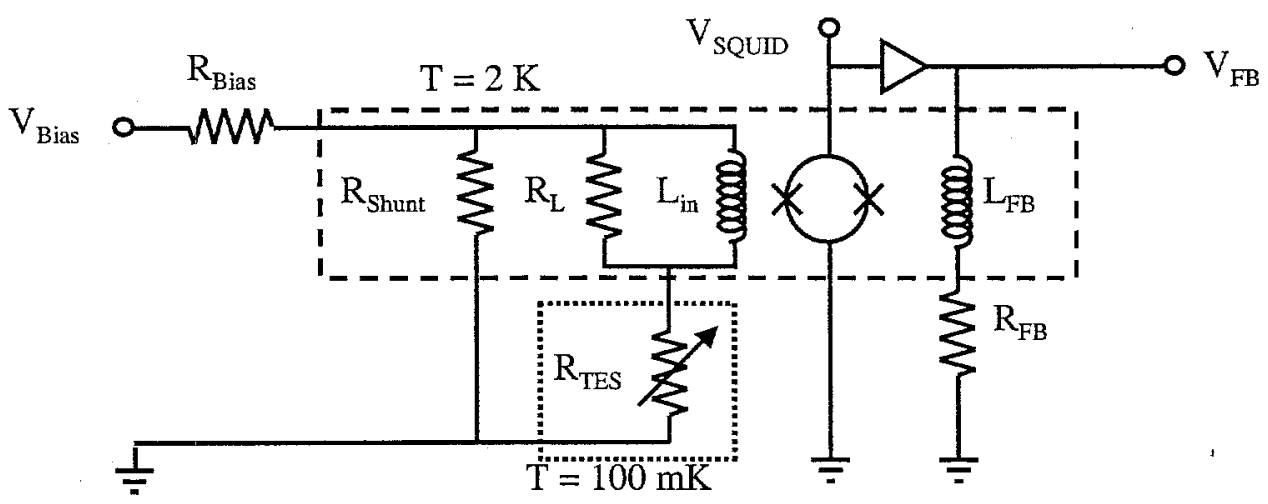

Fig. 6.5 Schematic of voltage bias circuit with parallel shunt and de SQUID read-out. Typical values of $R_{\text {Bias }}=2 \mathrm{k} \Omega, R_{\text {Shunt }}=10-20 \mathrm{~m} \Omega, R_{L}=20 \Omega, R_{F B}=1 \mathrm{k} \Omega$, and $\mathrm{L}_{\mathrm{in}}=0.25 \mu \mathrm{H}$. Dashed line encloses components that are at $2 \mathrm{~K}$. Dotted encloses the TES at $100 \mathrm{mK}$.

Eq. 6.4

$$
V_{\text {TES }}=\left(\frac{V_{\text {Bias }}}{R_{\text {Bias }}}-I_{T E S}\right) R_{\text {Shunt }},
$$

Most of the bias and SQUID circuits are at $2 \mathrm{~K}$, while the TES is at $\sim 100 \mathrm{mK}$. The bias and the feedback resistors are at room temperature and can be changed easily to accommodate different dynamic ranges.

We can simplify this bias circuit to the simple circuit shown in Fig. 3.2 by use of Thévenin's theorem, where

Eq. 6.5

$$
V_{\text {Th }}=V_{\text {Bias }} \frac{R_{\text {Shunt }}}{R_{\text {Bias }}+R_{\text {Shunt }}}, R_{\text {Th }}=\frac{R_{\text {Bias }} R_{\text {Shunt }}}{R_{\text {Bias }}+R_{\text {Shunt }}}
$$


For the typical resistance values given, $R_{T h} \approx R_{\text {Shunt }}$ and $V_{T h} \approx V_{\text {Bias }} R_{\text {Shunt }} / R_{\text {Bias }}$. Because the TES is connected as a two-wire measurement, there may be lead resistance in series with the TES. This lead resistance can be included into $R_{T h}$ for all intents and purposes.

\subsubsection{ADR operation}

To reach the operating temperature of $\sim 100 \mathrm{mK}$, we use an adiabatic demagnetization refrigerator (ADR) built into a liquid helium (LHe) cryostat. A schematic cross section of our ADR cryostat is shown in Fig. 6.6. The cryostat is a series of three nested shells. The shells are at room temperature of $300 \mathrm{~K}$, liquid nitrogen (LN) temperature of $77 \mathrm{~K}$, and pumped LHe temperature of $\sim 2 \mathrm{~K}$, respectively. The $77 \mathrm{~K}$ and $2 \mathrm{~K}$ shells have reservoir tanks with the respective liquid cryogen. Each shell is thermally isolated from the other shells by low thermal conductivity supports and vacuum space. The outer shells shield the inner shells from blackbody radiation. The actual ADR is nested within the 2 K shell.

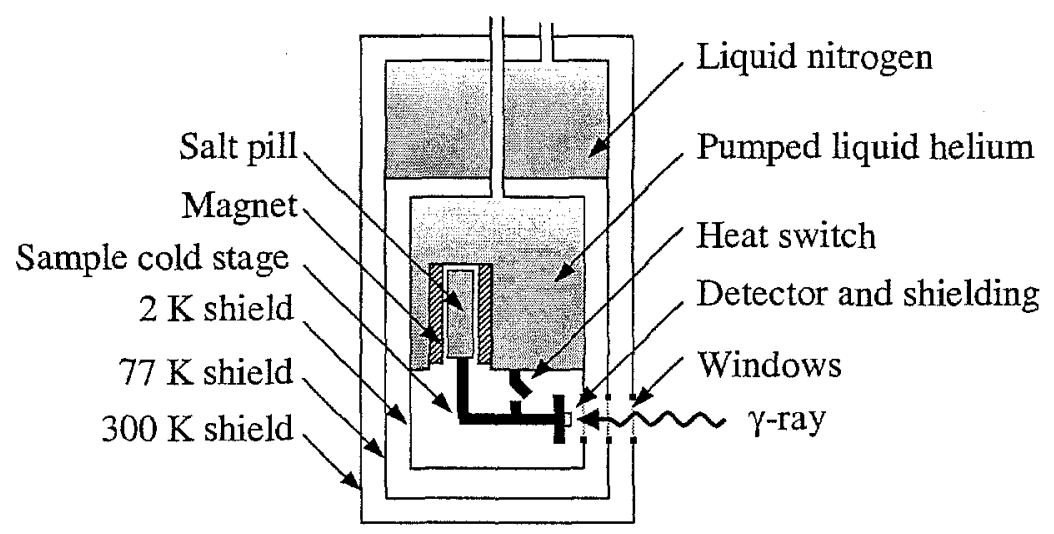

Fig. 6.6 Schematic cross section of the adiabatic demagnetization refrigerator. 
Refrigeration by magnetic cooling was first proposed by Debye in 1926. Magnetic cooling is possible in a paramagnetic material with magnetic moment $\mu$ and an entropy $S(T, B)$ that is dependent on temperature and magnetic field. At high temperatures, the electronic magnetic moments of the ions are completely disordered, with entropy $\left.S\right|_{T \rightarrow \infty, B=0}=R \ln (2 J+1)$, where $J$ is the total angular moment and $R=8.315 \mathrm{~J} \mathrm{~K}^{-1} \mathrm{~mole}^{-1}$ is the ideal gas constant. At low temperatures, the interactions of the magnetic moments become greater than the thermal energy, allowing the magnetic moments to orient themselves and reduce disorder. Similarly, at any given temperature, an applied magnetic field will also partially orient the moments and decrease the entropy [6]. This is the mechanism for magnetic cooling.

In our ADR, we use ferric ammonium alum (FAA, $\left.\mathrm{Fe}_{2}\left(\mathrm{SO}_{4}\right)_{3} \cdot\left(\mathrm{NH}_{4}\right)_{2} \mathrm{SO}_{4} \cdot 24 \mathrm{H}_{2} \mathrm{O}\right)$ as the paramagnetic salt, with $J=5 / 2$. The FAA salt pill is suspended inside a superconducting electromagnet. The magnet is conduction cooled by the $2 \mathrm{~K}$ stage. The sample cold stage and the salt pill are thermally coupled to the LHe bath by a mechanical heat switch. Fig. 6.7 shows the entropy of FAA as a function of temperature and applied magnetic field. The FAA is pre-cooled to $2 \mathrm{~K}$, shown as point $a$ in Fig. 6.7. A magnetic field is applied to magnetize the FAA isothermally (line $a b$ in Fig. 6.7). The heat of magnetization released by this process is

Eq. 6.6

$$
Q\left(T_{i}\right)=n T_{i} \int_{0}^{B_{i}}\left(\frac{\partial S}{\partial B}\right)_{T_{i}} d B
$$




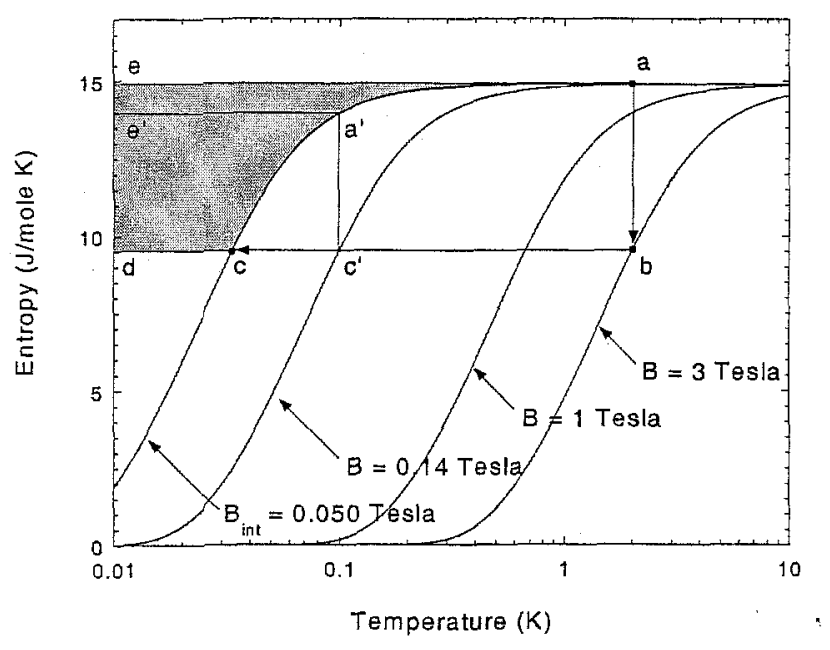

Fig. 6.7 Entropy of FAA salt pill for different applied magnetic fields.

The rectangular area abde is the heat of magnetization. The shaded area is the cooling power of the salt pill after demagnetization. The rectangle area e'a'c'd is the cooling power while regulated at $T=100 \mathrm{mK}$.

where $n$ is the number of moles, $T_{i}=2 \mathrm{~K}$ is the initial temperature, and $B_{i}=3$ Tesla is the applied magnetic field. This heat is absorbed by the LHe bath. Then, the FAA is thermally decoupled from the LHe bath by opening the heat switch. Once isolated, the applied magnetic field is quasistatically reduced to zero such that the entropy does not change, thus the term "adiabatic demagnetization" (line $b c$ in Fig. 6.7).

For non-interacting moments, the magnetic energy is $\varepsilon=\mu B$. The partition function has the form $Z \sim e^{-\varepsilon / k T}$. The entropy is effectively a function of only $B / T$. For the adiabatic process, we have

$$
S\left(\frac{B_{i}}{T_{i}}\right)=S\left(\frac{B_{f}}{T_{f}}\right) \Rightarrow \frac{T_{f}}{B_{f}}=\frac{T_{i}}{B_{i}},
$$


where $T_{f}$ and $B_{f}$ is the final temperature and magnetic field, respectively. The applied external magnetic field can be reduced to zero. However, the FAA has an internal field of $B_{\text {int }}=50 \mathrm{mTesla}$ which limits the base temperature of the cooling[7]. For small fields where the internal field is comparable, the effective field is $B_{f}^{2}=B_{e x t}{ }^{2}+B_{i n t}{ }^{2}$, where $B_{e x t}$ is the externally applied field. For a 3 Tesla initial field at $2 \mathrm{~K}$, the lowest temperature attainable is $\sim 33 \mathrm{mK}$. In practice, the base temperature is limited to $\sim 60 \mathrm{mK}$ by thermal loading of the electrical wiring and structural supports from the FAA cold stage to the 2 $\mathrm{K}$ shell. For more details on magnetic refrigeration, please refer to Timbie [8].

Thermal loading on the ADR must be minimized to achieve a low base temperature and long hold time. All electrical wiring from the ADR cold stage to the $2 \mathrm{~K}$ shell uses either low thermal conductivity phosphor bronze wires or superconducting CuNi-clad $\mathrm{NbTi}$ wires. For components which are at the same temperature, we use $\mathrm{Cu}$ or $\mathrm{Cu}$ clad $\mathrm{NbTi}$ wires to ensure good electrical and thermal conductivity.

The heat load gradually disorders the paramagnetic salt, warming it up along the entropy line at constant $B_{\text {int }}$. shown in Fig. 6.7. The cooling energy of the FAA is given by

Eq. 6.8

$$
Q\left(B_{f}\right)=n \int_{T_{f}}^{\infty} T\left(\frac{\partial S}{\partial T}\right)_{B_{f}} d T
$$

which is significantly smaller than the heat of magnetization. To maintain an operating temperature greater than the base temperature, the ADR can be regulated by application of a small magnetic field. The cooling energy is given by 
Eq. 6.9

$$
Q\left(T_{R}\right)=n T_{R} \int_{B_{i}}^{B_{f}}\left(\frac{\partial S}{\partial B}\right)_{T_{R}} d B
$$

where $T_{R}$ is the regulated temperature. Depending on the value of $T_{R}$, this cooling power may be greater than Eq. 6.8. For a typical thermal load of several $\mu \mathrm{W}$, the hold time of the ADR below $100 \mathrm{mK}$ is approximately 6-8 hours. Once the heat load has driven the base temperature above the range of interest, the adiabatic process can be repeated by closing the mechanical heat switch and coupling the ADR to the LHe cold bath, returning the state of the salt pill to point $a$ in Fig. 6.7.

\subsubsection{Data acquisition}

In our experimental set-up, most equipment is interfaced to a personal computer by GPIB (IEEE 488.2) standard. Digital data is acquired and stored using LabVIEW programming language. Only three parameters can be measured: $V_{B i a s}, V_{F B}$, and $T_{B a t h}$. All other parameters are either taken from physical dimensions of the microcalorimeter or derived from these three measurable quantities.

The temperature of the $\mathrm{ADR}$ is sensed by a Ge resistance thermometer (GRT) with a typical resistance of $\sim 2 \mathrm{k} \Omega$ and sensitivity $d R / d T \sim-50 \Omega / \mathrm{mK}$ at $100 \mathrm{mK}$. The resistance of the GRT is read by a resistance bridge (Oxford Instruments AVS-47) with typical excitation voltage of $30 \mu \mathrm{V}$. The bridge outputs the resistance as a proportional voltage to a digital voltmeter with GPIB interface to the computer, where it is converted to a 
temperature value. Temperature regulation is provided by a manual set point on the resistance bridge with a voltage output which is proportional to the difference between the resistance and a manual set point. This difference output is passed through a proportional integrating and differentiating (Linear Research LR-130) controller then applied to the ADR electromagnet to heat the salt pill.

For acquisition of IV curves, the bias voltage is applied with a function generator sweeping at $0.1 \mathrm{~Hz}$. The sweep frequency must be slow such that the microcalorimeter is in thermal equilibrium at all times. The bias voltage $V_{\text {Bias }}$ and the resultant SQUID feedback voltage $V_{F B}$ are recorded by a digital oscilloscope (Sony RTD 710, 10-bit resolution, $100 \mathrm{MHz}$ sampling rate) controlled by computer via GPIB interface.

For acquisition of gamma-ray pulse data, we digitize the pre-trigger baseline, the rise time, the peak, and the tail out to several decay times. The bias voltage is applied by a battery. The $V_{F B}$ pulse signals are amplified and low-pass filtered (Ithaco 4302) at the Nyquist frequency of the sampling rate to prevent high frequency noise from being aliased into the measurement bandwidth. After anti-aliasing, the signals are recorded by the digital oscilloscope controlled by computer via GPIB interface.

For sensitive electrical measurements, electronic pick-up of radio-frequency (rf) and 60 $\mathrm{Hz}$ noise is a concern. Whenever possible, equipment should be powered by batteries to avoid $60 \mathrm{~Hz}$ ripple. Multiply grounded experimental setups create "ground loops", which generate noise current. Generally, the lowest noise configuration is to ground the setup at 
only one point. One must take care to avoid electric shock, especially with high voltage devices such as computer monitors and other CRTs. If necessary, the entire experiment can be placed inside a grounded Faraday cage to further reduce pick-up. Fig. 6.8 shows the grounding scheme for a typical experimental setup. Electronic equipment that requires $60 \mathrm{~Hz}$ ac power share a common ground, but is grounded to earth only at one point, typically at the computer monitor or at the Faraday cage. Dc SQUTD and TES bias are applied with batteries.

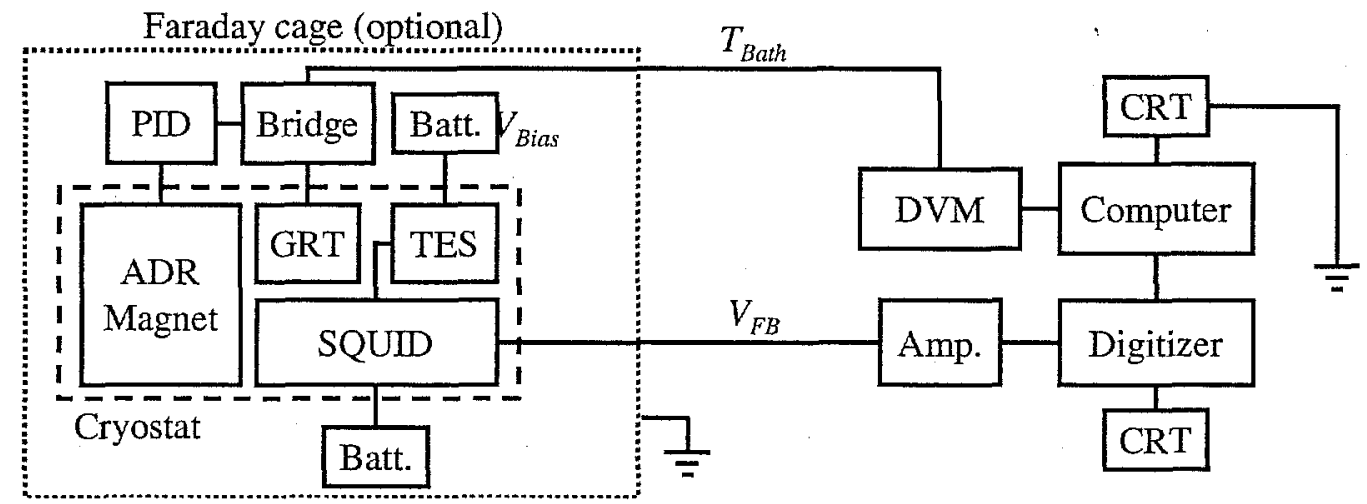

Fig. 6.8 Diagram of ground connections in typical experimental setup.

Solid lines indicate ground connections between equipment. Dashed line indicates the cryostat container. Dotted line indicates optional grounded Faraday cage. Also shown are the signal lines for $V_{B i a s}, V_{F B}$, and $T_{\text {Bath }}$.

The metallic body of the cryostat generally acts as an imperfect Faraday cage in shielding the interior from external electric fields due to non-conductive gaps and openings such as o-ring seals. The SQUWDs are sensitive to magnetic fields. They are shielded in superconducting $\mathrm{Nb}$ tubes. The TES is sensitive to magnetic fields and are shielded in a Kryoperm cover. Wiring to the TES are magnetically shielded in thin superconducting $\mathrm{Pb}$ tubes. The $\mathrm{ADR}$ magnet is shielded with vanadium permador to reduce stray fields at the cold stage. 


\section{References}

[1] A. F. Bello, T. Van Buuren, J. E. Klepeis and T. W. Barbee, Jr., Mat. Res. Soc. Proc., 524, 279 (1998).

[2] Emerson \& Cuming Specialty Polymers, 46 Manning Rd., Billerica, MA 01821, USA.

[3] Goodfellow Corporation, 800 Lancaster Ave., Berwyn, PA 19312-1780, USA

[4] T. Van Duzer, C.W. Turner, Principles of Superconductive Devices and Circuits, $2^{\text {nd }} E d$., Prentice Hall (1999).

[5] Hypres, Inc., 175 Clearbrook Rd., Elmsford, NY 10523, USA.

[6] F. Pobell, Matter and Methods at Low Temperatures, Springer, New York (1996).

[7] C. Hagmann, P.L. Richards, Cryogenics, 35, 303 (1995).

[8] P.T. Timbie, G.M. Bernstein, P.L. Richards, Cryogenics, 30, 271 (1990). 
Chapter 7

\section{DATa Analysis}

In this project, many detectors were fabricated and tested. The complete understanding of the microcalorimeter comes from the analysis of the different devices and at different bias conditions. Three devices in particular, designated as "SnTES\#6", "SnTES\#7", and "SnTES\#8", have produced our best results. Primary focus will be directed at data taken from these microcalorimeters.

\subsection{Current-voltage characteristics}

Measuring the IV characteristics is a good way to begin characterizing the microcalorimeter. Fig. 7.1 shows a family of IV curves for SnTES\#7. Each IV curve is taken at a different $T_{\text {Bath }}$ ranging from the base temperature of $60 \mathrm{mK}$ to $100 \mathrm{mK}$, where the TES is nearly normal. IV curves are traced under voltage bias from $R_{n}$ down to $R_{0} \sim$ $40 \mathrm{~m} \Omega$. At $40 \mathrm{~m} \Omega$, the TES is in a mixed bias rather than a strong voltage bias. When operated below $40 \mathrm{~m} \Omega$ at even modest bias power, the TES becomes unstable and will not self-regulate on the transition. 


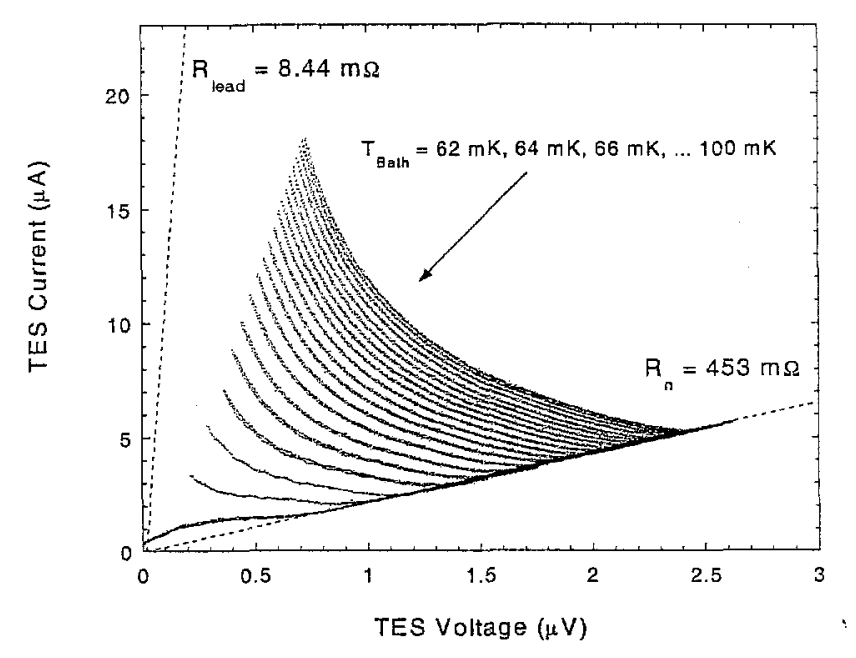

Fig. 7.1 Family of IV curves for SnTES\#7.

As expected, $I V$ curves traced at low $T_{B a t h}$ (high power) are further from the origin than IV curves traced at higher $T_{\text {Bath }}$ (low power). For SnTES\#7, we measured the TES normal resistance $R_{n}=453 \mathrm{~m} \Omega$ and a lead resistance $R_{\text {lead }}=8.44 \mathrm{~m} \Omega$. The lead resistance is measured by applying a small supercurrent while the TES is in the superconducting state and measuring the corresponding voltage drop.

\subsection{Thermal conductivity of SiN}

The IV curves are swept out slowly such that the microcalorimeter is in thermal equilibrium at all times. We can determine the resistance and dissipated power for every point on the IV curve. The TES bias power $P_{\text {Bias }}$, operating temperature $T_{0}$, and bath temperature $T_{B a t h}$ are different for every point in the family of IV curves. We extract values for $P_{B i a s}$ and $T_{B a t h}$ along lines of constant resistance ranging from $R_{0}=0.1 R_{n}$ to $0.9 R_{n}$ in steps of $0.1 R_{n}$ (Fig. 7.2). We expect these $P_{\text {Bias }}$ vs. $T_{B a t h}$ curves to be slightly 
offset in relation with each other because different $R_{0}$ correspond roughly to different $T_{0}$ due to the finite width of the transition.

We perform a least squares fit to each power curve using the general form

Eq. 7.1

$$
P_{\text {Bias }}=K_{S i N} \cdot\left(T_{0}^{N}-T_{\text {Bath }}^{N}\right) \text {. }
$$

We fit $P_{\text {Bias }}$ vs. $T_{B a t h}$ to find $K_{S i N}, N$, and $T_{0}$. We repeat for each set of $P_{B i a s}$ and $T_{B a t h}$ taken along lines of different constant $R_{0}$ ranging from $0.1 R_{n}$ to $0.9 R_{n}$. Fig. 7.3 shows the values of $N$ and $K_{S i N}$ found from fitting versus $R_{0}$.

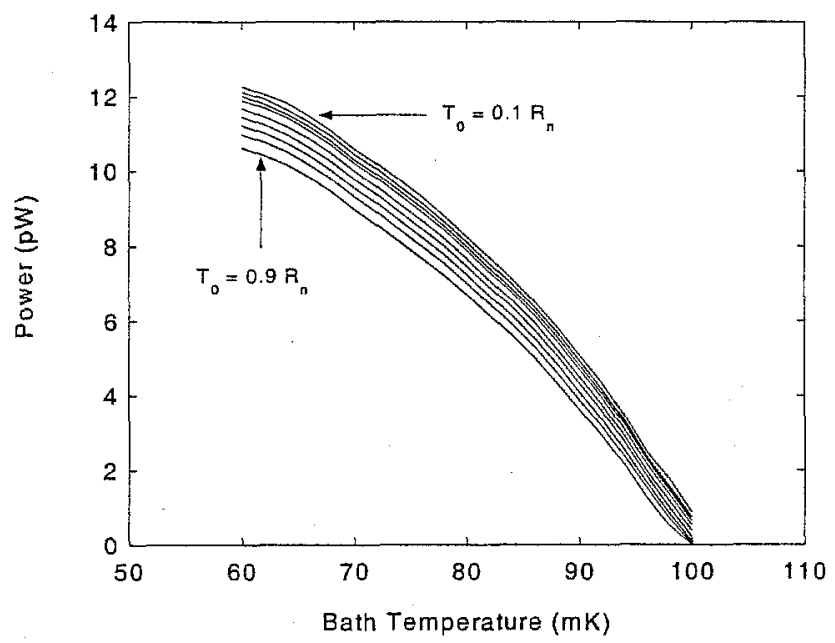

Fig. 7.2 TES $P_{\text {bias }}$ vs. $T_{\text {Bath }}$ for different $R_{0}$. 

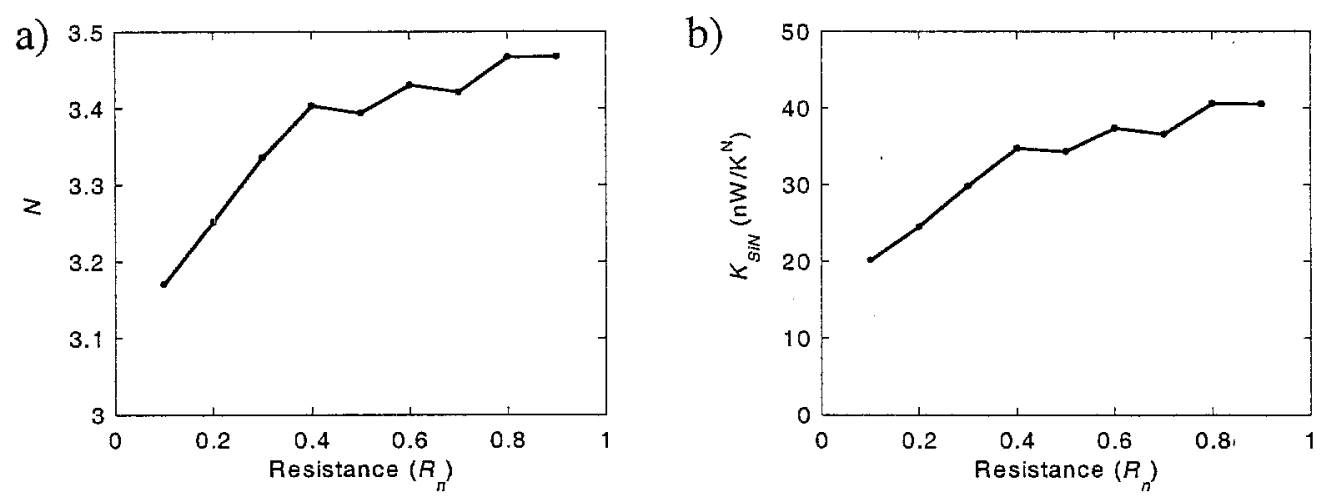

Fig. 7.3 SnTES\#7 IV curve fitted parameters.

We find the value of $N$ to changes from 3.17 at $0.1 R_{n}$ to 3.47 at $0.9 R_{n}$. Similarly, we find $K_{S i N}$ to change from $20.159 \mathrm{nW} / \mathrm{K}^{\mathrm{N}}$ to $40.562 \mathrm{nW} / \mathrm{K}^{\mathrm{N}}$ over the same range. We know that at low $R_{0}$, the TES current is high. Therefore, this dependence on $R_{0}$ suggests that there is a slight variation of the transition width with TES current. The values of $N$ differs slightly from the theoretical value of 4 . This difference may be due to membrane quality, membrane and TES geometry, and damage from microfabrication processing. We expect the values for $N$ and $K_{\text {SiN }}$ found at the highest $R_{0}$ (i.e., lowest TES current) to be closest to the true values. This approximation assumes that at the highest $R_{0}$ (lowest TES current) there is negligible broadening of the TES transition width such that $R_{0}$ is at the same $T_{0}$ regardless of current.

Given the values of $N$ and $K_{\text {SiN }}$ at low current, we plot the thermal conductivity of the SiN $G_{S i N}$ as a function of temperature. Fig. 7.4 shows the measured $G_{S i N}$ compared with the theoretical specular and dirty limits as given by Holmes, et al. [1]. We see that our SiN is comparable to the specular limit. 


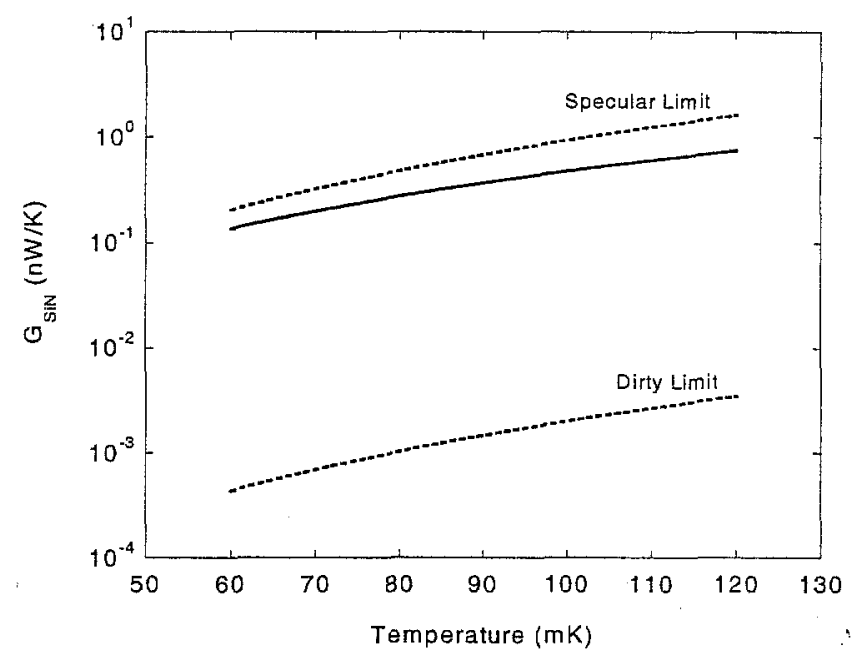

Fig. 7.4 Thermal conductivity of SiN for SnTES\#7.

$:$

\subsection{Superconducting transition}

The resistance and power of each point on the IV curves are easily found. With the fitted values of $K_{S i N}$ and $N$, we can estimate the operating temperature $T_{0}$ of any point on the IV curves. We can plot the superconducting transition of the TES, shown in Fig. 7.5. We see that IV curves at taken at different $T_{B a t h}$ give consistent transitions, except at very low resistances where the TES current is much higher at low $T_{\text {Bath }}$ than at high $T_{B a t h}$. For the IV curve traced at $60 \mathrm{mK}$, the current $\sim 18 \mu \mathrm{A}$ at $R_{0} \sim 40 \mathrm{~m} \Omega$. The IV curve traced at 92 $\mathrm{mK}$ has a current of $\sim 8 \mu \mathrm{A}$ at the same resistance. This suggests that there is a slight broadening of the transition at high currents. We also see a slight hysteresis in the transition, despite the IV curves being traced at a frequency of $0.1 \mathrm{~Hz}$. 


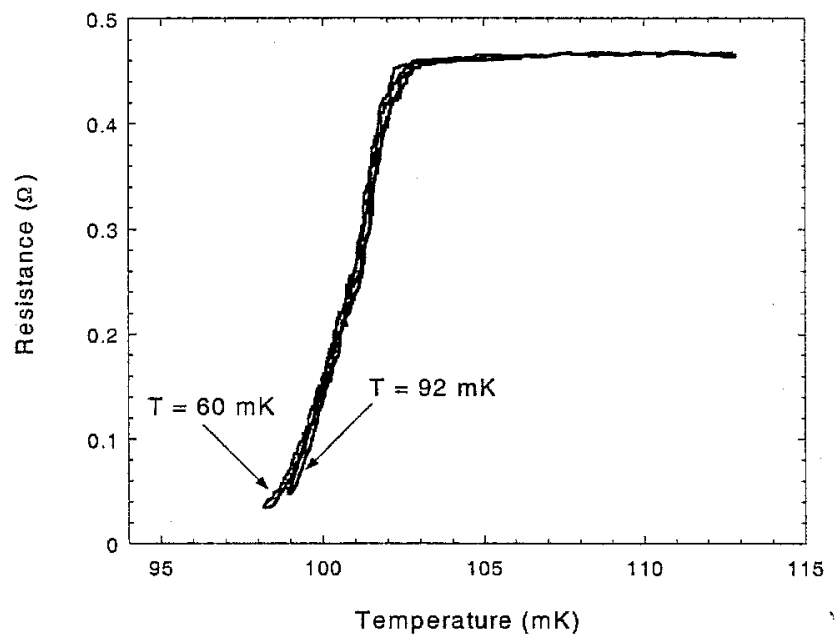

Fig. 7.5 Superconducting transition of the TES derived from IV analysis for SnTES\#7.

\subsection{Current dependence of the superconducting transition}

The current dependence of the superconducting transition can be examined by plotting the TES current against the TES temperature for the set of points in each measured IV curve, shown in Fig. 7.6. We can determine contours of constant resistance and superimposed them over the family of IV curves plotted in current-temperature space. If the superconducting transition has no current dependence, then the constant resistance contours will be parallel vertical lines. We see that the constant resistance contours have a small divergence, indicating that in the current range used here the resistance has a slight dependence on current as well as temperature. From extrapolation, we estimate that the transition width roughly doubles (from $\sim 2 \mathrm{mK}$ to $\sim 4.2 \mathrm{mK}$ ) as the current is increased by a factor of 10 (from $1.5 \mu \mathrm{A}$ to $15 \mu \mathrm{A}$ ). We know from the optimization calculations performed in Section 5.5.2.4 (page 107) that the signal-to-noise ratio is relatively unaffected even for a $100 \%$ increase in transition width if $T_{B a t h}$ fluctuations 
dominate the noise. Thus, our claim in Section 3.3 (page 46) that the current dependence on the superconducting transition may be neglected is validated.

\subsection{Iteration method for finding thermal conductivity of $\mathrm{SiN}$}

Recall in Section 7.2 (page 130) that our simple two-parameter fitting analysis for finding $N$ and $K_{S i N}$ is only valid for $P_{B i a s}$ vs. $T_{B a t h}$ taken at low current such that the broadening of the transition is negligible. This simple fit assumes that at each $R_{0}$, the TES temperature $T_{0}$ is constant, which is not true if current effects are not negligible. A more accurate approach to this problem is to perform a multivariate fit to Eq. 7.1 using $P_{\text {Bias }}, T_{B a t h}$, and $T_{0}$ as input variables to find $N$ and $K_{\text {SiN }}$. From our original IV curves, we have a series of data in $P_{\text {Bias }}$ and $T_{\text {Bath. }}$. The simple fitting process in Section 7.2 gives us an initial estimate of $N$ and $K_{S i N}$ which allows us to find $T_{0}$ at every point on the IV curves. We then perform a multivariate fit of $P_{B i a s}, T_{B a t h}$, and $T_{0}$ to find new values for $N$ and $K_{S i N}$. With the new $N$ and $K_{S i N}$, we can find a new set of $T_{0}$ again. We iterate the multivariate fitting process and observe the convergence of $N$ and $K_{S i N}$ to their true values. This iterative algorithm for finding $N$ and $K_{S i N}$ is currently under development at LLNL.

\subsection{Superconducting transition for constant $V_{\text {Bias }}$}

The superconducting transitions plotted in Fig. 7.5 are derived from IV curves traced at constant $T_{\text {Bath }}$ and near-constant $P_{\text {Bias. }}$. However, under voltage bias operation, the trajectory of the TES resistance is along constant bias voltage. Thus, it is the superconducting transition traced along constant bias voltage that is relevant to the understanding of the behavior of the TES. 


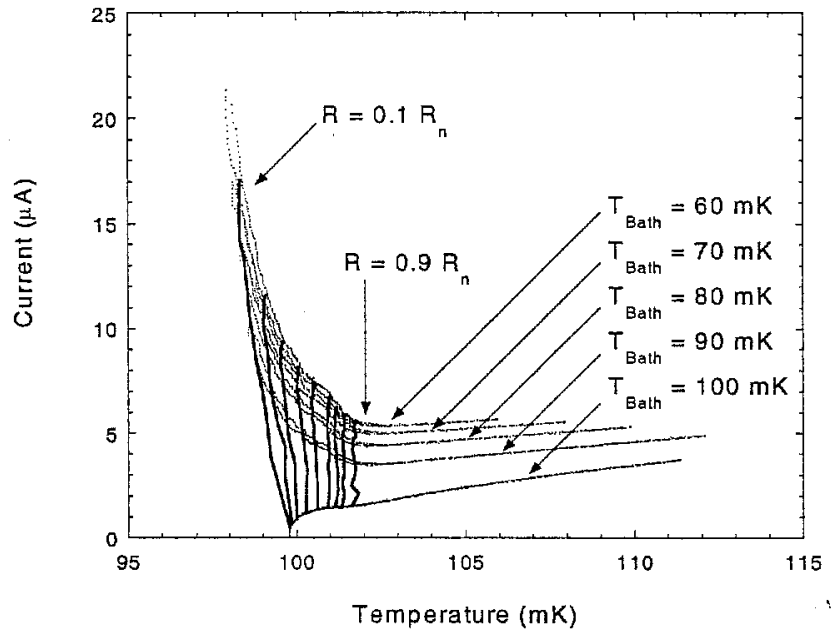

Fig. 7.6 Current dependence of the superconducting transition for SnTES\#7.

Fine dotted lines are IV curves at different $T_{B a t h}$ plotted in current-temperature space. Heavy dashed lines are contours of constant resistance.

Recall that in the TES bias circuit (Fig. 6.5) the low-impedance voltage bias is applied by use of $R_{\text {Bias }}$ and $R_{\text {Shunt }}$. For practical values of these resistors, the actual voltage across the TES can be made very stiff, but still imperfect. Fig. 7.7 shows load line for constant external $V_{\text {Bias }}$. We see that the TES voltage is not constant. We find the superconducting transition along this load line (Fig. 7.8). We see that the transition along the load line lies within the set of all transition points taken from all IV curves. We also note that at low resistance, the load line transition is closer to the low temperature side of the set than at high resistance. This suggests that the operating $\alpha$ along the load line transition may be lower than expected in this region. 


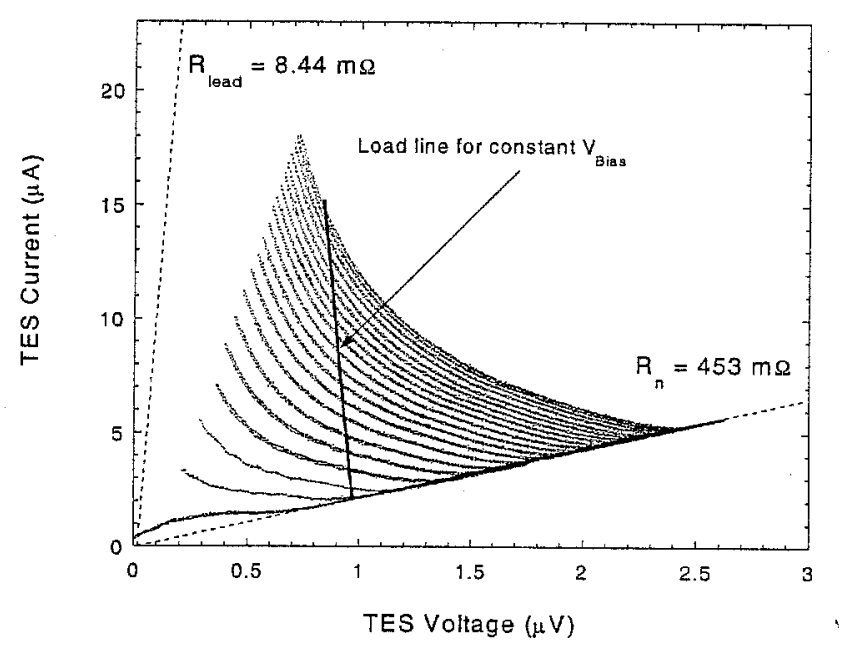

Fig. 7.7 SnTES\#7 load line for constant external $V_{\text {Bias }}=197.7 \mathrm{mV}, R_{\text {Bias }}=2 \mathrm{k} \Omega, R_{\text {Shuut }}=10 \mathrm{~m} \Omega$.

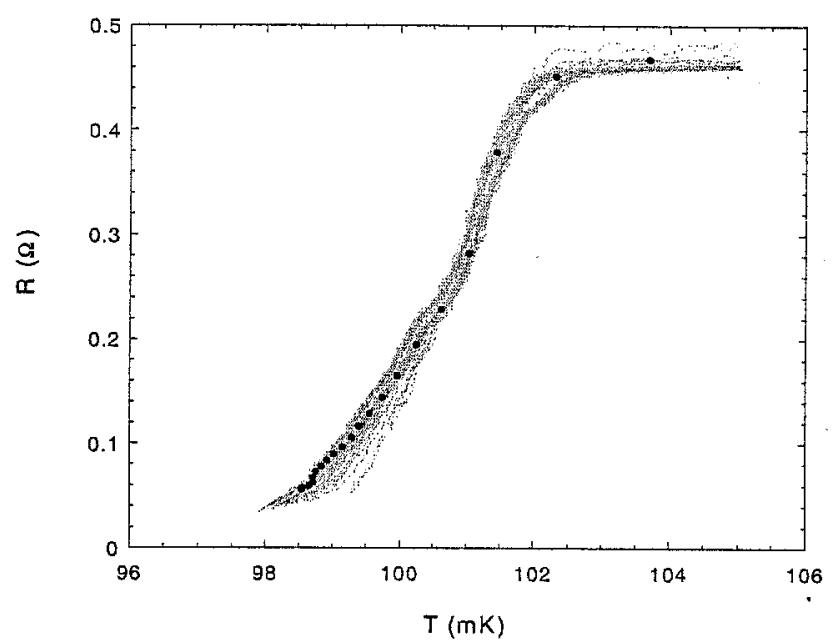

Fig. 7.8 SnTES\#7 transition along load line (black dots) compared with complete set (gray dots). 


\subsection{Device study: SnTES\#6}

\subsubsection{Bias point study}

We observed pulses at many different bias points for all detectors. In particular, we operated device SnTES\#6 at two sets of bias points: along a line of constant $R_{0}$ while varying $T_{B a t h}$, and along an IV curve at constant $T_{B a t h}$ while varying $R_{0}$ (Fig. 7.9). For the constant $R_{0}$ set, $R_{0}$ is $34 \mathrm{~m} \Omega$ and $T_{\text {Bath }}$ ranges from $70 \mathrm{mK}$ to $82 \mathrm{mK}$. For the constant $T_{\text {Bath }}$ set, $T_{\text {Bath }}$ is $70 \mathrm{mK}$ and $R_{0}$ ranges from $33.9 \mathrm{~m} \Omega$ to $193 \mathrm{~m} \Omega$. We see that this set of data deviates significantly from the constant power contour, indicating that the transition is wide. These two sets of bias points effectively spans the two independent parameters that we actually control in the operation of the detector.

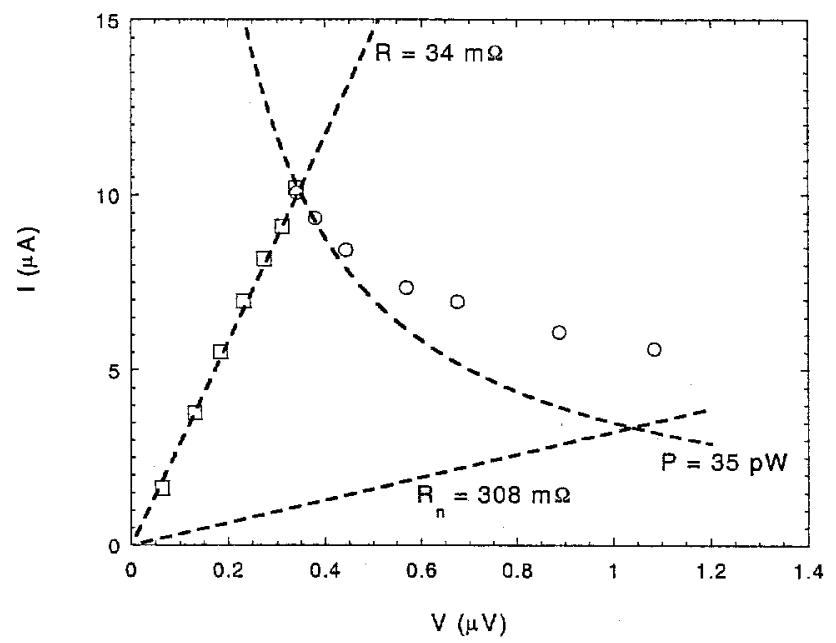

Fig. 7.9 Bias points for SnTES\#6.

Circles are bias points at varying $R_{0}$ with $T_{\text {Bath }}$ fixed at $70 \mathrm{mK}$. Squares are bias points at varying $T_{\text {Bath }}$ while keeping $R_{0}$ constant at $34 \mathrm{~m} \Omega$. Also shown are lines indicating constant $R_{n}$ and constant power.

For this specific device, we have limited IV data and cannot construct the superconducting transition using the methods described in Sections 7.2 through 7.6. 
However, we can use the two sets of bias points to find some of the crucial parameters necessary for approximating the transition.

From the constant $R_{0}$ bias points, we can plot $P_{B i a s}$ vs. $T_{B a t h}$ and extract the value of $G_{S i N}=$ $0.294 \mathrm{nW} / \mathrm{K}$, which is consistent with SnTES\#7. For this particular device, $G_{S i N}$ is effectively constant with $N=1$ and $K=G_{S i N}=0.294 \mathrm{nW} / \mathrm{K}$ over the temperature range of interest, which is comparable with SnTES\#7 at the same small range of temperatures. The resistance and temperature of each bias point is calculated. The constant $T_{\text {Bath }}$ set of bias points map out the superconducting transition, shown in Fig. 7.10. We note that the transition for SnTES\#6 is extremely broad. The transition width is $\sim 20 \mathrm{mK}$ wide, whereas the transition width of SnTES\#7 is approximately $4 \mathrm{mK}$ wide.

We fit a straight line through this set of points. Using the linear fit as the transition, we simulate the detector response to a gamma ray for all bias points. The numerical model is compared to real measured pulses. The heat capacities and thermal conductivities used in the model are calculated from the physical dimensions with significant margins of error. The values for the heat capacities and thermal conductivities are adjusted within the error limits such that the simulated signal response agrees reasonably with measurement. Because the superconducting transition is very wide, the bias temperatures, and subsequently many other temperature-dependent parameters, span a wide range of values. Table 7.1 lists all relevant parameters used to describe SnTES\#6. For some parameters, we give two values, one at for bias point lowest on the transition and one for the bias point highest on the transition. 


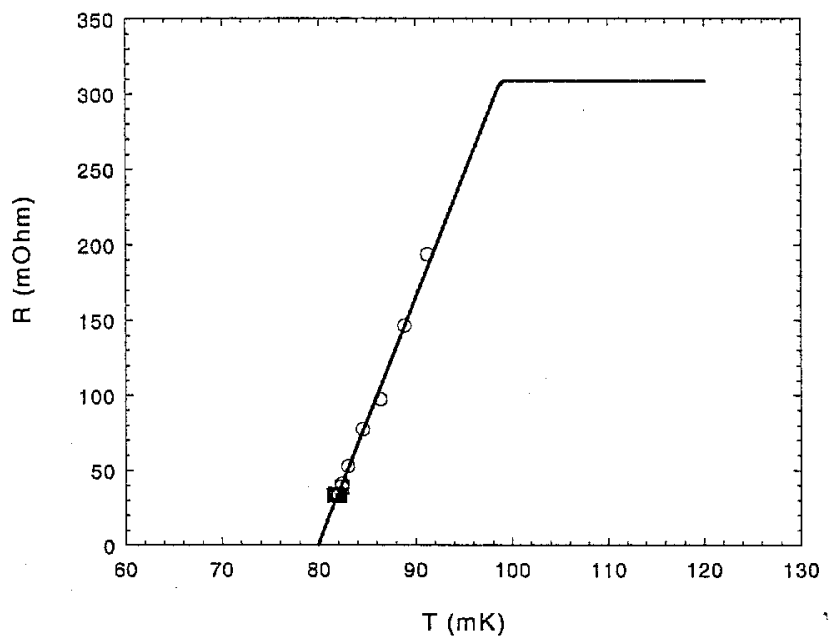

Fig. 7.10 Superconducting transition mapped out by bias points of SnTES\#6.

Circles are constant $T_{\text {Bath }}$ bias points. Squares are constant $R_{0}$ bias points. Solid line is fitted transition. The value of $R_{n}$ is found from IV data not shown.

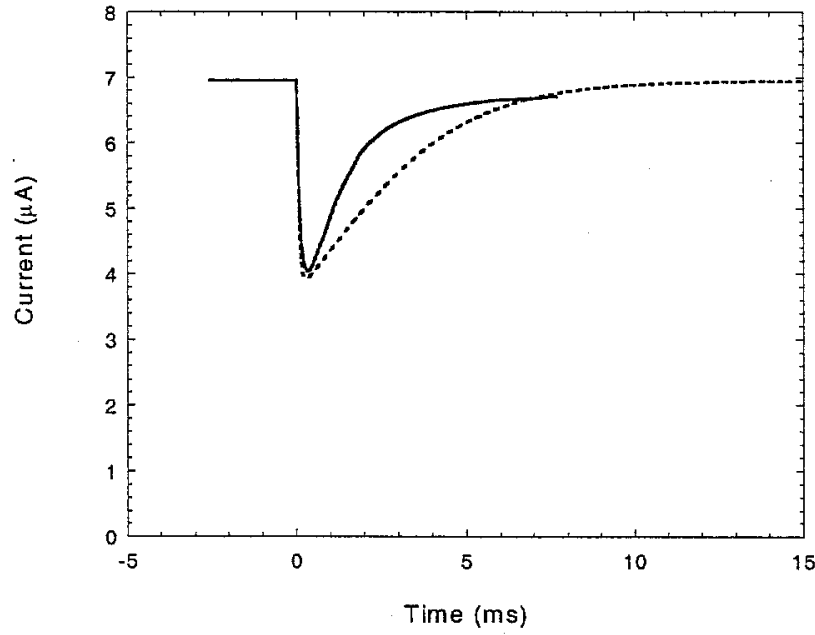

Fig. 7.11 Typical $60 \mathrm{keV}$ signal for SnTES\#6.

Solid line is measured pulse. Dotted line is modeled pulse. 


\begin{tabular}{|c|c|c|c|c|}
\hline & \multirow[b]{2}{*}{ Parameter } & \multirow[b]{2}{*}{ Description } & \multicolumn{2}{|c|}{ Value } \\
\hline & & & $\begin{array}{l}\text { Bottom of } \\
\text { transition }\end{array}$ & $\begin{array}{c}\text { Top of } \\
\text { transition }\end{array}$ \\
\hline \multirow{5}{*}{ 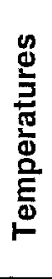 } & $T_{0}$ & Operating & $81.9 \mathrm{mK}$ & $91.1 \mathrm{mK}$ \\
\hline & $T_{T h}$ & Bias resistor & \multicolumn{2}{|c|}{$-2 K$} \\
\hline & $T_{\text {Bath }}$ & Bath & $69.5-81.9 \mathrm{mK}$ & $69.5 \mathrm{mK}$ \\
\hline & $T_{C}$ & Transition & \multicolumn{2}{|c|}{$79.9 \mathrm{mK}$} \\
\hline & $\Delta T_{C}$ & Transition width & \multicolumn{2}{|c|}{$20.7 \mathrm{mK}$} \\
\hline \multirow{4}{*}{$\begin{array}{l}0 \\
\infty \\
0\end{array}$} & $C_{A b s(\mathrm{eff})}$ & Effective absorber & $1.54 \mathrm{keV} / \mathrm{mK}$ & $1.94 \mathrm{keV} / \mathrm{mK}$ \\
\hline & $C_{\text {TES(eff) }}$ & Effective TES & $7.19 \mathrm{keV} / \mathrm{mK}$ & $8.01 \mathrm{keV} / \mathrm{mK}$ \\
\hline & $G_{S i N}$ & SiN membrane & \multicolumn{2}{|c|}{$0.294 \mathrm{nW} / \mathrm{K}$} \\
\hline & $G_{A b s(e f f)}$ & Effective Stycast & $2.53 \mathrm{nW} / \mathrm{K}$ & $3.45 \mathrm{nW} / \mathrm{K}$ \\
\hline \multirow{4}{*}{ 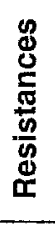 } & $R_{\text {Shunt }}$ & TES Shunt & \multicolumn{2}{|c|}{$20 \mathrm{~m} \Omega$} \\
\hline & $R_{n}$ & TES (normal) & \multicolumn{2}{|c|}{$360 \mathrm{~m} \Omega$} \\
\hline & $R_{0}$ & TES (operating) & $34.3 \mathrm{~m} \Omega$ & $195 \mathrm{~m} \Omega$ \\
\hline & $R_{\text {lead }}$ & Lead & \multicolumn{2}{|c|}{$12.7 \mathrm{~m} \Omega$} \\
\hline \multirow{4}{*}{$\frac{g}{m}$} & $V_{T h}$ & Bias voltage & $0.118 \mu \mathrm{V}$ & $1.27 \mu \mathrm{V}$ \\
\hline & $l_{0}$ & Steady-state current & $10.2 \mu \mathrm{A}$ & $1.63 \mu \mathrm{A}$ \\
\hline & $P_{0}$ & Bias power & $0.105-3.63 p W$ & $6.10 \mathrm{pW}$ \\
\hline & $\alpha$ & Transition "steepness" & 41.5 & 8.14 \\
\hline \multirow{2}{*}{$\stackrel{0}{\underline{O}}$} & $E$ & Gamma ray energy & \multicolumn{2}{|c|}{$60 \mathrm{keV}$} \\
\hline & $I_{\text {SQUID }}$ & SQUID noise & \multicolumn{2}{|c|}{$10 \mathrm{pA} / \mathrm{Hz}^{1 / 2}$} \\
\hline
\end{tabular}

Table 7.1 SnTES\#6 values used for numerical model.

(The ranges of $T_{\text {Bath }}$ and $P_{0}$ at the bottom of the transition correspond to the constant $R_{0}$ set of bias points.)

Heat capacities and thermal conductivities are treated as constants during a pulse. Fig. 7.11 shows a typical measured pulse compared with the numerical model. We see the pulse height agrees well and the decay time is significantly different. This is true for all bias points of SnTES\#6. Fig. 7.12 and Fig. 7.13 shows the pulse heights and decay times for the constant $R_{0}$ bias points and the constant $T_{B a t h}$ bias points, respectively. 

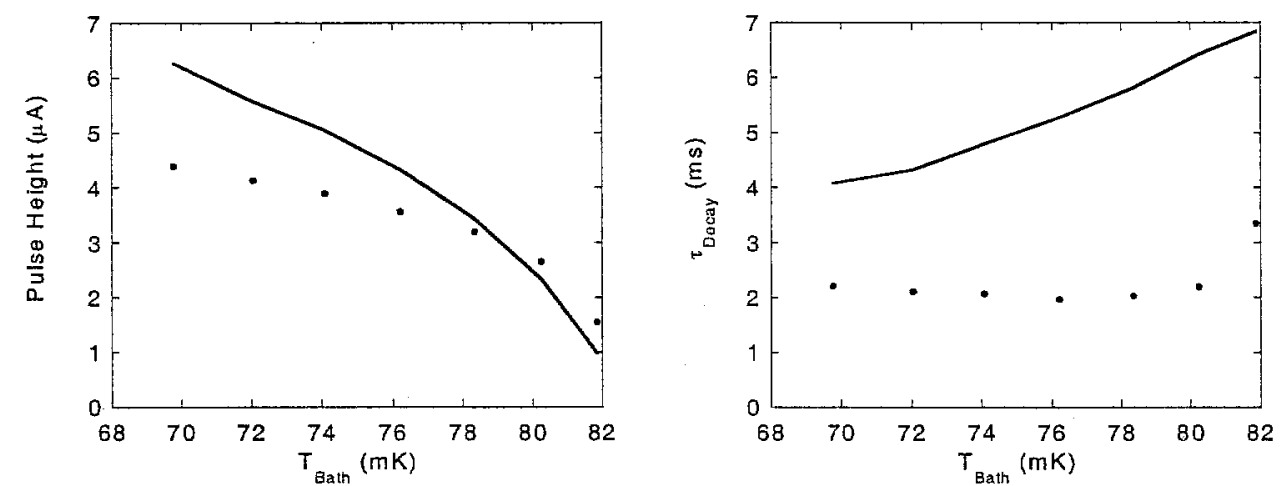

Fig. 7.12 Response of SnTES\#6 for constant resistance bias points. Points are measured pulse heights and decay times, lines are numerical calculations.

We see that modeled and measured pulse heights agree reasonably. Note that the largest deviations are at low $R_{0}$ and low $T_{B a t h}$. We also see that the modeled and measured decay times differ by nearly a factor of two and do not agree very well.

We speculate that the differences between data and model arise from our crude estimate of the superconducting transition. The insufficient IV data do not allow us to construct a complete temperature- and current-dependent superconducting transition. However, recall in Fig. 7.8 that we saw a decrease in $d R / d T$ near the bottom of the superconducting transition along a load-line. If this is also true for SnTES\#6, it may contribute to decreased signal height near the bottom of the transition. However, this hypothesis does not explain the discrepancy in the decay times. 

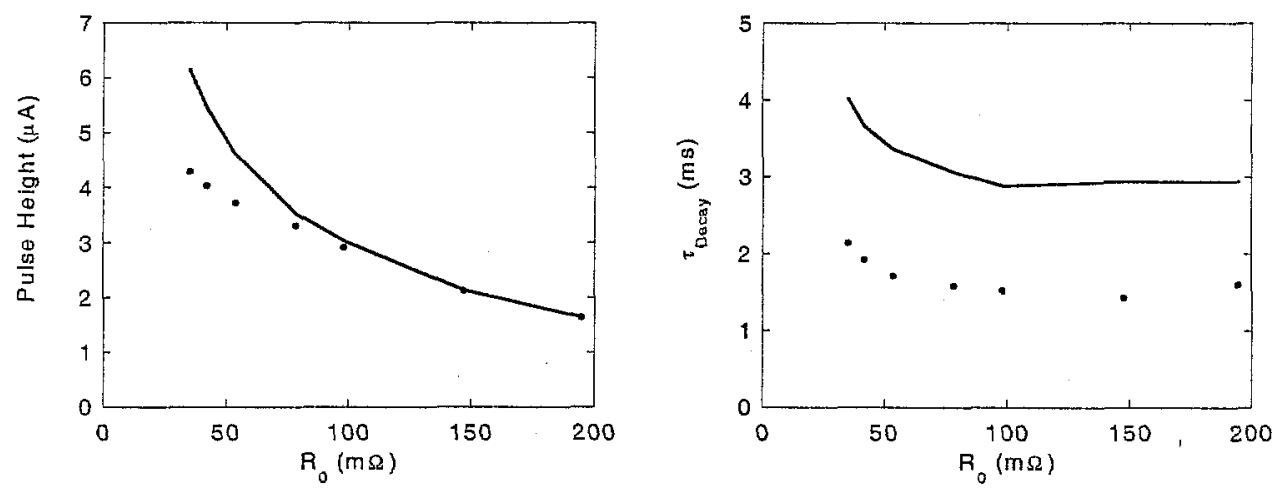

Fig. 7.13 Response of SnTES\#6 for constant $T_{\text {Bath }}$ bias points. Points are measured pulse heights and decay times, lines are numerical calculations.

The modeled response of the detector can be made to agree with measurement more closely by using the following parameters: $C_{A b s(e f f)}=0.5 \mathrm{keV} / \mathrm{mK}, G_{A b s(e f f)}=55 \mathrm{pW} / \mathrm{K}$, $C_{T E S(e f f)}=3 \mathrm{keV} / \mathrm{mK}$. The detector response with these values give pulse heights and decay times that agree with measurement to within $30 \%$ for all bias points. However, these numbers are physically unrealistic. For example, to achieve the above value for $G_{A b s(e f f)}$, the dimensions of the Stycast epoxy would have to be $10 \mu \mathrm{m}$ thick and $15 \mu \mathrm{m}$ in diameter.

Therefore, we attribute the errors between measurement and model to incomplete knowledge of the superconducting transition. Note that for later devices (SnTES\#7 described in Section 7.8, page 147 and SnTES\#8 described in Section 7.9, page 156), we do have complete knowledge of the superconducting transition, and the corresponding results show excellent agreement between measurement and model. 


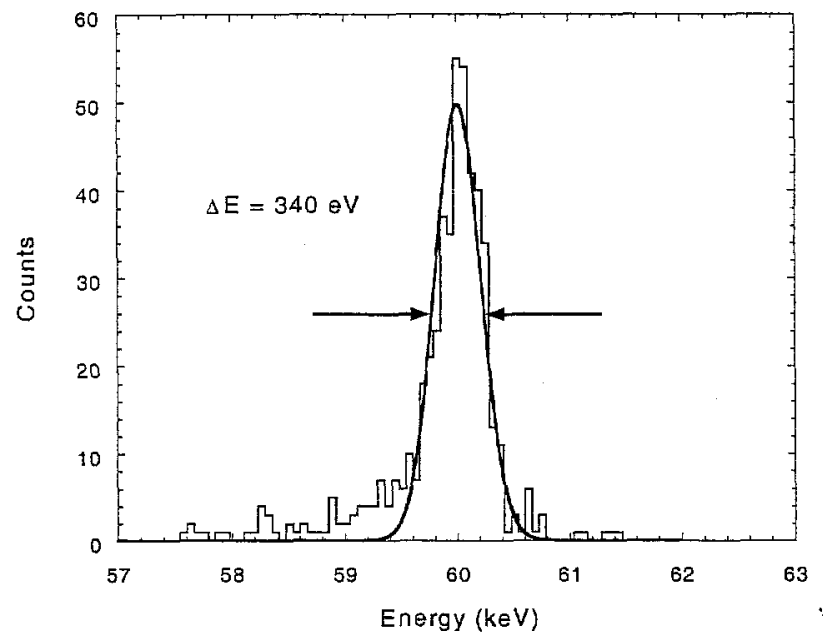

Fig. $7.1460 \mathrm{keV}$ spectral peak measured by SnTES\#6 with energy resolution of $340 \mathrm{eV}$. Histogram is counts in each energy bin. Solid line is gaussian fit of histogram data.

\subsubsection{Energy resolution and noise analysis}

We measured pulse heights and noise spectra at all bias points. The digitized data length is 1024 points at a sampling time of $10 \mu$ s, which corresponds to a sampling rate of 100 $\mathrm{kHz}$. Higher frequency components are removed with an anti-aliasing filter at $50 \mathrm{kHz}$. The optimal bias point of this microcalorimeter is in the region of low $T_{B a t h}$ and low $R_{0}$. The best energy resolution was found at $T_{B a t h}=70 \mathrm{mK}$ and $R_{0}=43 \mathrm{~m} \Omega$. The spectral peak for $60 \mathrm{keV}$ is shown in Fig. 7.14. The observed energy resolution is $340 \mathrm{eV}$ FWHM, which is comparable with the fundamental limit for Ge ionization detectors.

The corresponding measured noise spectrum is shown in Fig. 7.15 with theoretical noise contributions. For comparison, we show phonon noise calculated from the one-element model (Section 3.5, page 52) as well as the two-element model (Section 3.6, page 59). The maximum frequency is limited by the sampling rate to $50 \mathrm{kHz}$. The frequency 
resolution is about $100 \mathrm{~Hz}$ and is determined by the digitizing length. At frequencies below $500 \mathrm{~Hz}$, the dominant noise source is $60 \mathrm{~Hz}$ pick-up and its harmonics, which are unresolved at the sampling rate used. The magnitude of the $60 \mathrm{~Hz}$ noise and its harmonics is estimated from a noise spectra measured with high resolution at low frequencies which is not shown. The Johnson noise from the TES shunt resistor is dominant over the remaining range of the noise spectrum. This can be remedied by cooling the shunt resistor from $2 \mathrm{~K}$ to $100 \mathrm{mK}$, thereby reducing the Johnson noise by a factor of $\sqrt{2} 2$, or approximately 4.5 . In searching for the largest pulse height, we operated the TES under high $\alpha$ at $R_{0}=43 \mathrm{~m} \Omega$. However, at this resistance, we do not have a strong voltage bias, but rather a mixed bias. This resulted in higher Johnson noise at low frequency due to poor electrothermal feedback. The SQUID amplifier noise varies with each experiment, depending on the quality of the V- $\Phi$ characteristic. SQUID noise spectrum was not measured directly in the experimental setup, so we assumed it to be flat with frequency based on previous measurements. Overall, the theoretical noise spectrum is lower than the measured noise spectrum.

The signal pulse height is $4.03 \mu \mathrm{A}$. Each noise source is integrated in quadrature from $100 \mathrm{~Hz}$ to $50 \mathrm{kHz}$. The total measured noise integrated in quadrature from $100 \mathrm{~Hz}$ to 50 $\mathrm{kHz}$ is $8.60 \mathrm{nA}$. The signal-to-noise ratio is 470 , giving an energy resolution of $300 \mathrm{eV}$ FWHM at $60 \mathrm{keV}$. This is comparable to the actual measured linewidth of $340 \mathrm{eV}$ FWHM, suggesting that there is only minor peak broadening due to bath temperature fluctuations, position dependence, and other causes which do not appear in the noise spectrum. 


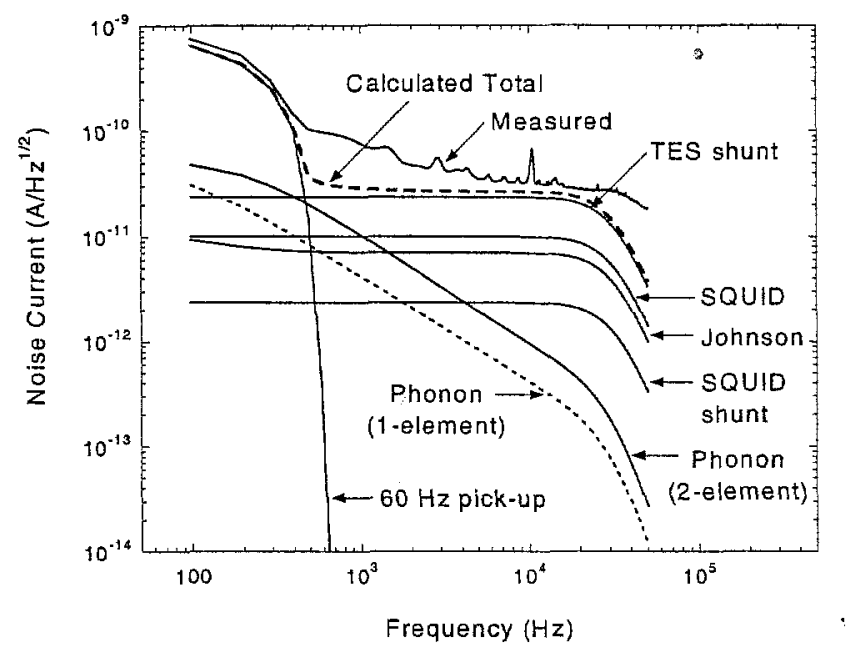

Fig. 7.15 Noise spectrum of SnTES\#6 compared with theoretical noise contributions.

Table 7.2 summarizes the integrated noise currents and the corresponding spectral linewidth contribution for each noise source. The total calculated noise is roughly equal to the total measured noise, indicating that we have used a valid set of noise models.

For comparison, we also estimate the phonon and Johnson noise by the 2-element numerical simulations described in Section 5.4 (page 95). We find that the modeled phonon noise current is $2.33 \mathrm{nA}$ and the modeled Johnson noise current is $0.305 \mathrm{nA}$. It is interesting to note that the 2-element numerical phonon noise is three times greater than the 2-element analytical phonon noise. We attribute this discrepancy to poor estimation of the numerical model parameters and superconducting transition shape. 


\begin{tabular}{|c|c|c|}
\hline Noise Source & Current (rms) & Linewidth (FWHM) \\
\hline Phonon (2-element analytical) & $0.799 \mathrm{nA}$ & $27.9 \mathrm{eV}$ \\
\hline Johnson & $1.14 \mathrm{nA}$ & $40.0 \mathrm{eV}$ \\
\hline TES Shunt & $3.84 \mathrm{nA}$ & $134 \mathrm{eV}$ \\
\hline SQUID & $1.62 \mathrm{nA}$ & $56.8 \mathrm{eV}$ \\
\hline SQUID Shunt & $0.38 \mathrm{nA}$ & $13.3 \mathrm{eV}$ \\
\hline 60 Hz Pick-up & $8.22 \mathrm{nA}$ & $288 \mathrm{eV}$ \\
\hline Calculated Total & $9.30 \mathrm{nA}$ & $326 \mathrm{eV}$ \\
\hline Measured Total & $8.60 \mathrm{nA}$ & $300 \mathrm{eV}$ \\
\hline
\end{tabular}

Table 7.2 SnTES\#6 noise contributions.

We also compare the integrated noise currents (theoretical and measured) for both sets bias points (constant $T_{0}$ and constant $R_{0}$ ) (Fig. 7.16). We see that for all bias points, the theoretical noise is reasonable agreement with measured noise. We also see that the largest signal-to-noise is found at low $T_{B a t h}$ and low $R_{0}$, as we claimed at the beginning of this section.

\subsection{Device study: SnTES\#7}

The low transition temperature and broad transition width of SnTES\#6 yielded relatively small signal sizes. SnTES\#7 was fabricated with a higher target $T_{c}$. The superconducting transition was measured from IV characteristics and shown in Fig. 7.5. This device appears to be suitable for our needs. 

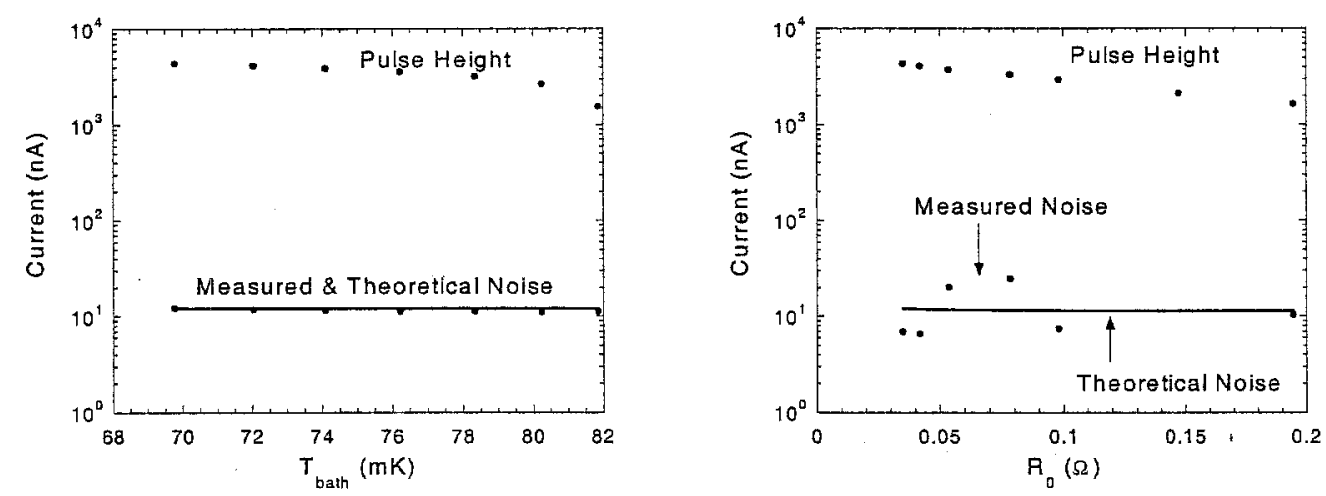

Fig. 7.16 Measured noise, theoretical noise, and pulse height at different bias points.

\subsubsection{Energy range and non-linearity}

Using the method described at the end of the previous section, we identified an optimal bias point. We measured pulses from a wide range of energies. The ${ }^{241} \mathrm{Am}$ source produces $59.54 \mathrm{keV}$ and $26.34 \mathrm{keV}$ gamma rays. Am decays into the daughter product $\mathrm{Np}$, which emits characteristic L-shell x rays at $11.9 \mathrm{keV}, 13.95 \mathrm{keV}, 16.93 \mathrm{keV}, 17.75$ $\mathrm{keV}$, and $20.8 \mathrm{keV}$. These $\mathrm{x}$ rays also excite a $\mathrm{Cu}$ fluorescence line $(8.04 \mathrm{keV})$ from the detector mount. The $60 \mathrm{keV}$ gamma rays interact with the $\mathrm{Sn}$ absorber via photoelectric absorption which is sometimes followed by the emission of $\mathrm{Sn} \mathrm{K}$ lines at $25.5 \mathrm{keV}$ and $28.9 \mathrm{keV}$, thus creating secondary escape lines in the spectrum at $34.5 \mathrm{keV}$ and $31.1 \mathrm{keV}$. Fig. 7.17 shows measured pulses for the energies described above. We see that all pulses have a "kink" at $\sim 3 \mu \mathrm{A}$. This is most likely the result of a non-linear artifact in the superconducting transition. This structure is finer than the noise of the IV curve measurements, thus we are unable to see the "kink" in the superconducting transition derived from IV curves. 
We modeled the response of this device over the range of energies previously described. Fig. 7.18 shows the linear approximation of the superconducting transition used to model SnTES\#7 and the corresponding bias point load line transition found from the $I V$ curves. The figure also shows the bias point of the pulses shown in Fig. 7.17 and the maximum excursion along the transition during the pulse. The parameters used in the numerical model are shown in Table 7.3. We see that $I_{0}$ and $\alpha$ are significantly greater than SnTES\#6, accounting for the larger pulse height.

Fig. 7.19 shows measured pulses compared with modeled pulses for several energies. We see that the model is in good agreement with data in terms of pulse height and decay time. Note that the largest deviation is at $60 \mathrm{keV}$.

Fig. 7.20 shows measured pulse heights and decay times compared with the numerical model. We see good agreement between the two. Note that the pulse height response to energy is non-linear. Recall from Section3.3 (page 46) that the fundamental assumption for the response of the TES is that the change in temperature and resistance is small for absorption of a photon. For SnTES\#7, the transition width and heat capacities are such that the change in temperature and resistance of the TES is large enough that the signal response is no longer linear. Also, the simple linear function does not fit well near the bottom of the superconducting transition. This may account for the differences between model and data for pulse height and decay times. Furthermore, the reduced pulse height at higher energies will affect the energy resolution. 


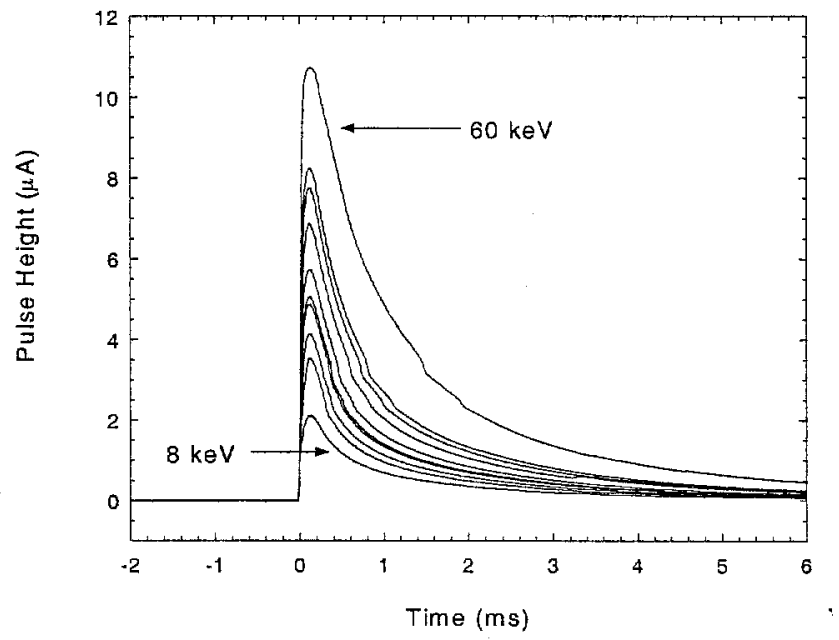

Fig. 7.17 SnTES\#7 measured pulses for $8 \mathrm{keV}$ to $60 \mathrm{keV}$ energies.

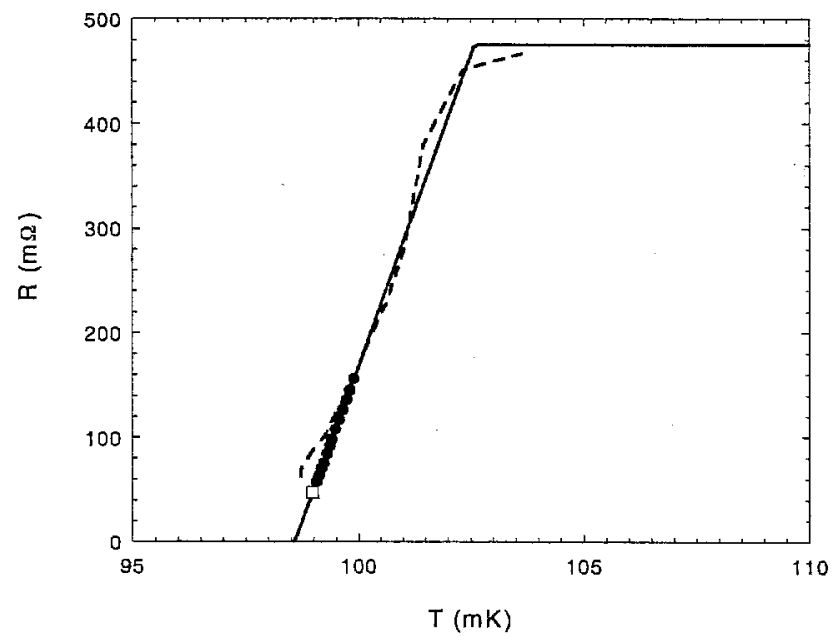

Fig. 7.18 Transition used in modeling SnTES\#7.

Solid is the linear transition used in the numerical model. Dashed line is transition along constant $V_{\text {Bias }}$ load line. The square indicates the bias point, and dots denote the maximum change in resistance of different energies between $8 \mathrm{keV}$ and $60 \mathrm{keV}$. 


\begin{tabular}{|c|c|c|c|}
\hline & Parameter & Description & Value \\
\hline \multirow{5}{*}{ 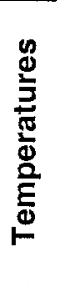 } & $T_{0}$ & Operating & $99.7 \mathrm{mK}$ \\
\hline & $T_{T h}$ & Bias resistor & $-2 K$ \\
\hline & $T_{\text {Bath }}$ & Bath & $65 \mathrm{mK}$ \\
\hline & $T_{C}$ & Transition & $98.6 \mathrm{mK}$ \\
\hline & $\Delta T_{c}$ & Transition width & $4 \mathrm{mK}$ \\
\hline \multirow{4}{*}{$\begin{array}{l}0 \\
\infty \\
0\end{array}$} & $C_{A b s(\text { eff })}$ & Effective absorber & $40 \mathrm{keV} / \mathrm{mK}$ \\
\hline & $C_{\text {TES }(\text { eff })}$ & Effective TES & $10 \mathrm{keV} / \mathrm{mK}$ \\
\hline & $G_{S i N}$ & SiN membrane & $0.29 \mathrm{nW} / \mathrm{K}$ \\
\hline & $G_{A b s(\text { eff })}$ & Effective Stycast & $25 \mathrm{nW} / \mathrm{K}$ \\
\hline \multirow{4}{*}{ 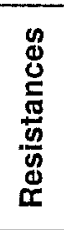 } & $R_{\text {Snumt }}$ & TES Shunt & $10 \mathrm{~m} \Omega$ \\
\hline & $R_{n}$ & TES (normal) & $453 \mathrm{~m} \Omega$ \\
\hline & $R_{0}$ & TES (operating) & $47.1 \mathrm{~m} \Omega$ \\
\hline & $R_{\text {lead }}$ & Lead & $8.44 \mathrm{~m} \Omega$ \\
\hline \multirow{4}{*}{ 兽 } & $V_{T h}$ & Bias voltage & $0.861 \mu \mathrm{V}$ \\
\hline & 10 & Steady-state current & $15.1 \mu \mathrm{A}$ \\
\hline & $P_{0}$ & Bias power & $10.7 \mathrm{pW}$ \\
\hline & $\alpha$ & Transition "steepness" & 250 \\
\hline \multirow{2}{*}{$\stackrel{0}{E}$} & $E$ & Gamma ray energy & $8-60 \mathrm{keV}$ \\
\hline & $I_{\text {SQUID }}$ & SQUID noise & $10 \mathrm{pA} / \mathrm{Hz}^{1 / 2}$ \\
\hline
\end{tabular}

Table 7.3 SnTES\#7 values used for numerical model. 

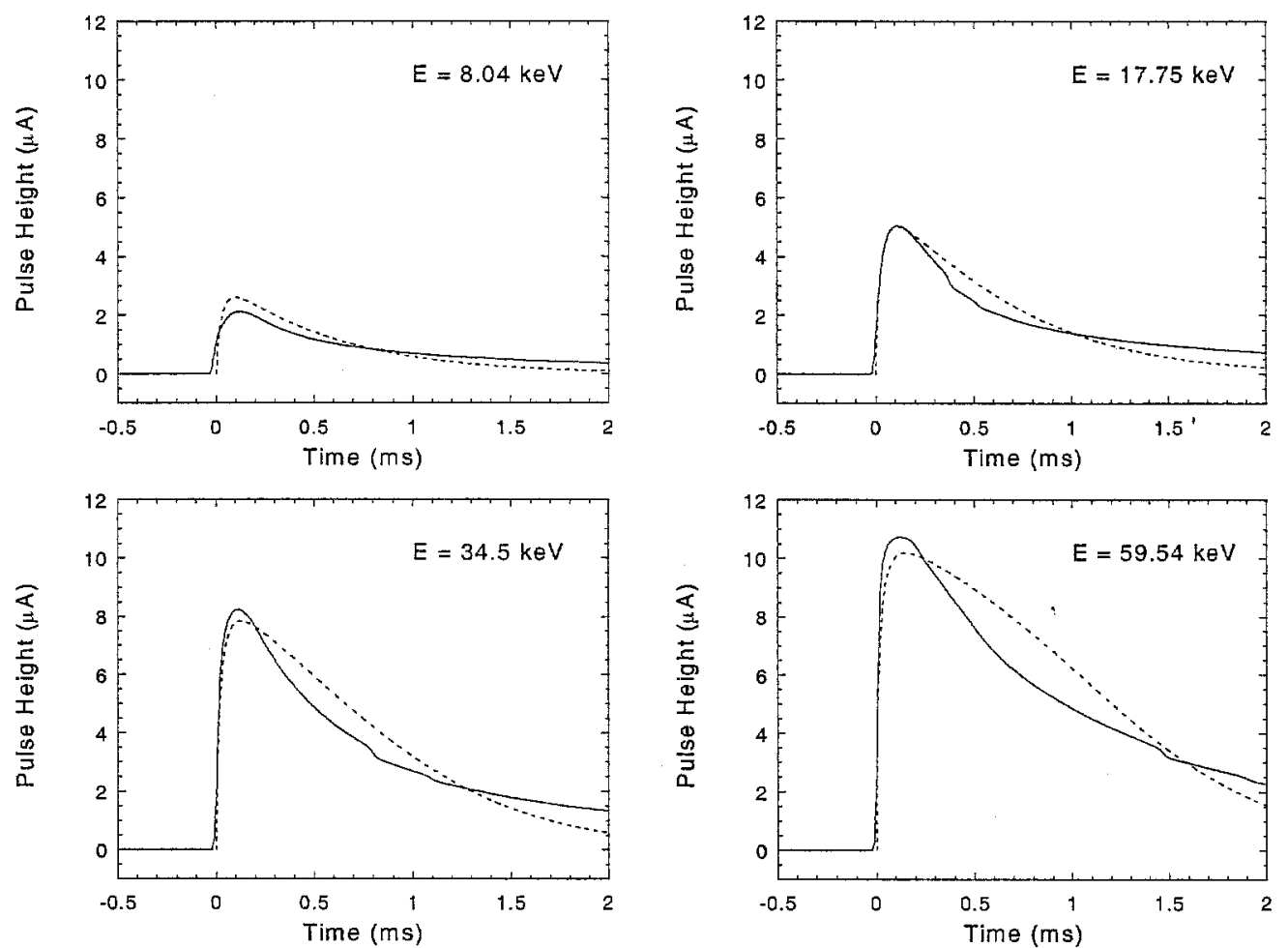

Fig. 7.19 SnTES\#7 measured (solid lines) and modeled (dotted lines) pulses for several energies.
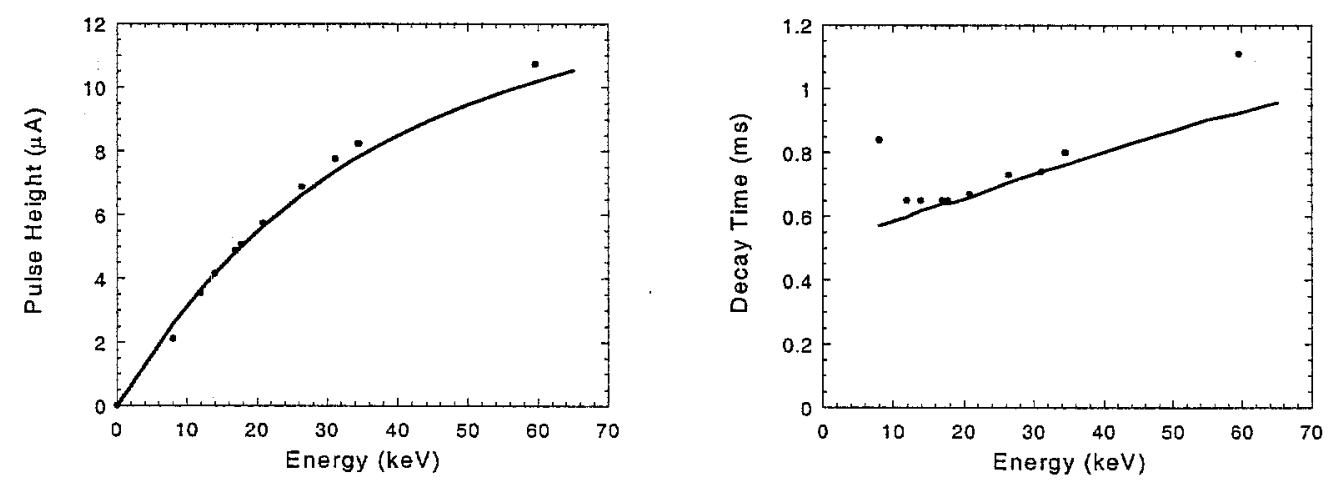

Fig. 7.20 SnTES\#7 pulse heights and decay times for measured (dots) and modeled (line) pulses. 


\subsubsection{Energy resolution and noise analysis}

The ${ }^{241} \mathrm{Am}$ spectrum measured by SnTES\#7 is shown Fig. 7.21. We observed the $60 \mathrm{keV}$ gamma rays from the ${ }^{241} \mathrm{Am}$ source, L-lines from the $\mathrm{Np}$ daughter product, $\mathrm{Sn}$ escape lines, and $\mathrm{Cu} \mathrm{K}$ fluorescence. The apparent linewidth at $60 \mathrm{keV}$ suggests an energy resolution of $120 \mathrm{eV} \mathrm{FWHM.} \mathrm{However,} \mathrm{because} \mathrm{of} \mathrm{the} \mathrm{non-linear} \mathrm{response} \mathrm{in} \mathrm{that}$ region, the true energy resolution is approximately $240 \mathrm{eV}$. Despite this degradation, we have improved the energy resolution over SnTES\#6 by choosing a higher $T_{c}$ and $T_{0}$. As the optimization of $T_{c}$ shows (Section 5.5.2.3, page 106), the advantages of increasing the operating power is greater than the disadvantages of the corresponding increase in heat capacity. SnTES\#7 also has, to its benefit, a sharper transition than SnTES\#6, although it is not fully understood how fabrication processes affect the transition width.

In Fig. 7.22 we show the noise spectrum of SnTES\#7 at the same bias point compared with calculated noise sources. Just as in SnTES\#6, we also compare the one-element phonon noise spectra with the two-element phonon noise spectra. For this device, we see a large difference between these two phonon noise models, with the two-element case agreeing well with the measured noise spectra. Again, we see $60 \mathrm{~Hz}$ pick-up dominating at frequencies below $500 \mathrm{~Hz}$. We observed $1 \mathrm{kHz}$ pick-up due to a nearby UHV turbo pump in our laboratory. But over the entire higher frequency range, the two-element phonon noise dominates due to a much large $G_{A b s}$ than in SnTES\#6. 


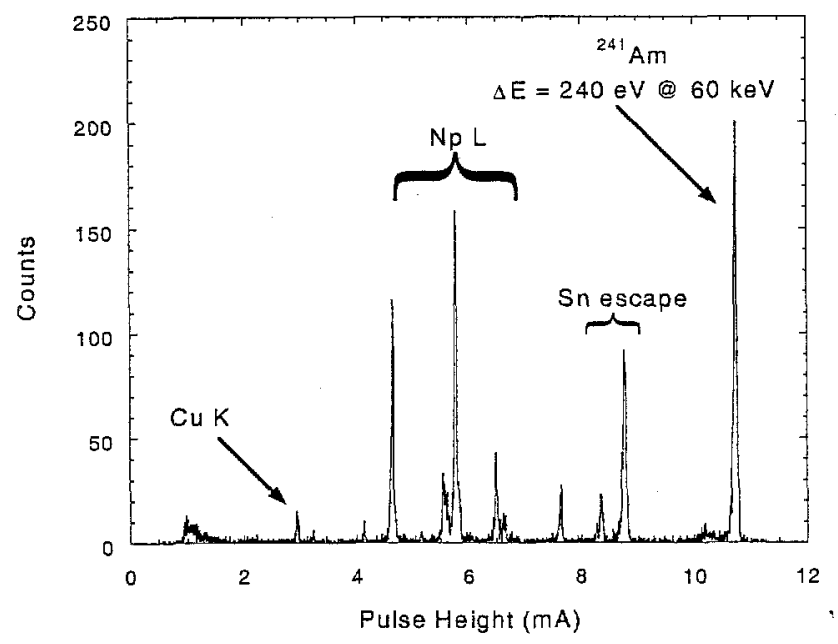

Fig. 7.21 SnTES\#7 spectrum with energy resolution of $240 \mathrm{eV}$ at $60 \mathrm{keV}$ degraded by non-linear gain.

The pulse data is acquired with a sampling rate of $100 \mathrm{kHz}$ with an anti-aliasing low-pass filter at $50 \mathrm{kHz}$. The signal pulse height is $10.73 \mu \mathrm{A}$. As before, each noise source is integrated in quadrature from $100 \mathrm{~Hz}$ to $50 \mathrm{kHz}$. Table 7.4 summarizes the integrated noise currents and the corresponding spectral linewidth contribution for each noise source.

We also simulated the phonon and Johnson noise currents using our numerical model. We find the phonon noise current to be $5.29 \mathrm{nA}$ and the Johnson noise current to be 0.348 $\mathrm{nA}$. We see that the multi-element numerical phonon noise is comparable to the integrated two-element analytical phonon noise, which is in good agreement with the measured noise. 


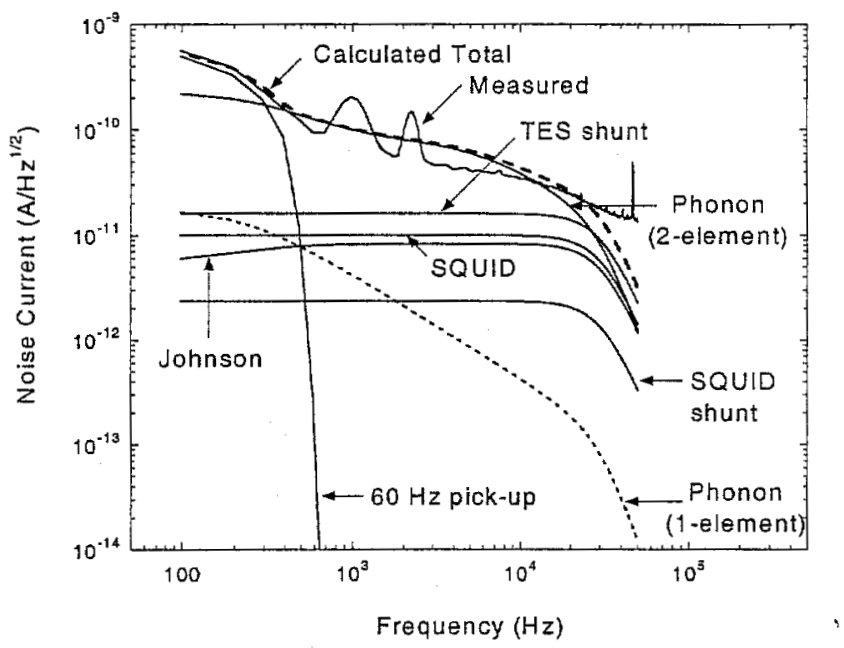

Fig. 7.22 Noise spectrum of SnTES\#7 compared with theoretical noise contributions.

The total measured noise integrated in quadrature from $100 \mathrm{~Hz}$ to $50 \mathrm{kHz}$ is $8.80 \mathrm{nA}$, giving us a signal-to-noise ratio of 1220 , which agrees with the measured apparent spectral line width without non-linear gain considerations.

\begin{tabular}{|c|c|c|}
\hline Noise Source & Current (rms) & Linewidth (FWHM) \\
\hline Phonon (2-element analytical) & $6.767 \mathrm{nA}$ & $88.9 \mathrm{eV}$ \\
\hline Johnson & $1.34 \mathrm{nA}$ & $17.7 \mathrm{eV}$ \\
\hline TES Shunt & $2.60 \mathrm{nA}$ & $34.2 \mathrm{eV}$ \\
\hline SQUID & $1.62 \mathrm{nA}$ & $21.3 \mathrm{eV}$ \\
\hline SQUID Shunt & $0.38 \mathrm{nA}$ & $5.00 \mathrm{eV}$ \\
\hline 60 Hz Pick-up & $6.29 \mathrm{nA}$ & $82.6 \mathrm{eV}$ \\
\hline Calculated Total & $9.83 \mathrm{nA}$ & $129 \mathrm{eV}$ \\
\hline Measured Total & $8.80 \mathrm{nA}$ & $116 \mathrm{eV}$ \\
\hline
\end{tabular}

Table 7.4 SnTES\#7 noise contributions. . 


\subsection{Device study: SnTES\#8}

SnTES\#8 was fabricated and chosen for its extremely sharp transition. The superconducting transition derived from IV characteristics is shown in Fig. 7.23. We see that in the region between $80 \mathrm{~m} \Omega$ and $350 \mathrm{~m} \Omega$ the transition is very sharp. The nonlinear "step" between $0.3 \Omega$ and $0.4 \Omega$ is believed to be caused by localized TES film stress exerted by the Stycast epoxy. It is known that film stress will alter $T_{c}$. Localized film stress will alter $T_{c}$ locally, creating a region with a different $T_{c}$. Effectively, this can seen as a two-step transition, where one of the steps correspond to the region of the film under stress.

\subsubsection{Energy range and linearity}

We modeled the response of SnTES\#8 over the range of measured energies. The parameters used in the numerical model are shown in Table 7.5. For the transition, the analytic function is an arctangent plus linear slope qualitatively fitted to the load line transition in the region above the bias point and below $0.3 \Omega$, which is the highest resistance reached during a pulse. Because of the non-linear transition, we take $d R$ and $d T$ to be the total change in resistance and temperature during a pulse for consideration of $\alpha$. Therefore, $\alpha$ takes on a range of values depending on the photon energy. For this particular transition, $\alpha$ scales with the photon energy. 


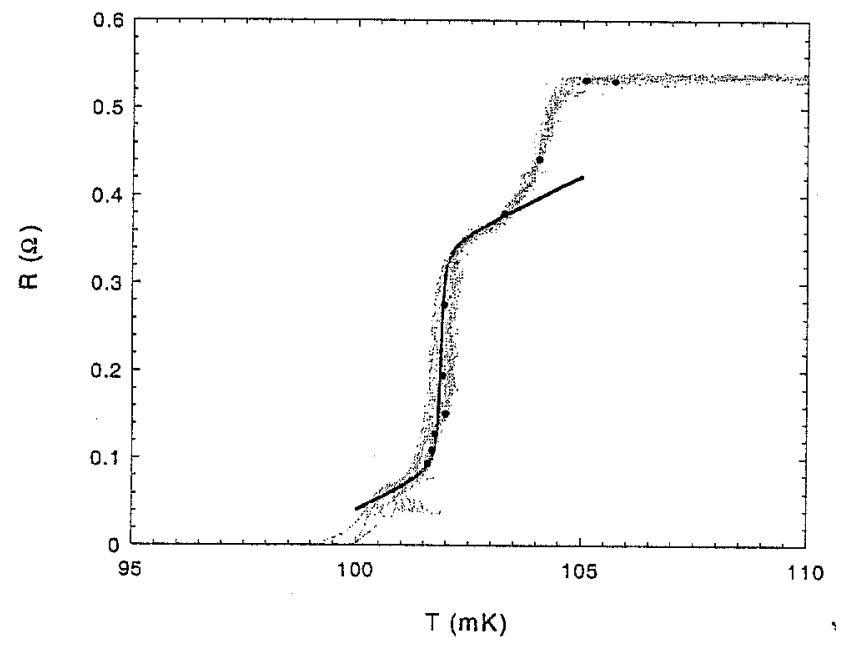

Fig. 7.23 SnTES\#8 superconducting transition derived from IV curve at $T_{B a t h}=65 \mathrm{mK}$.

Fig. 7.24a shows a measured $60 \mathrm{keV}$ pulse compared with the numerical model with good agreement. Fig. $7.24 \mathrm{~b}$ shows a single $60 \mathrm{keV}$ pulse normalized on a logarithmic scale. We see that the signal-to-noise ratio is approximately $10^{4}$.
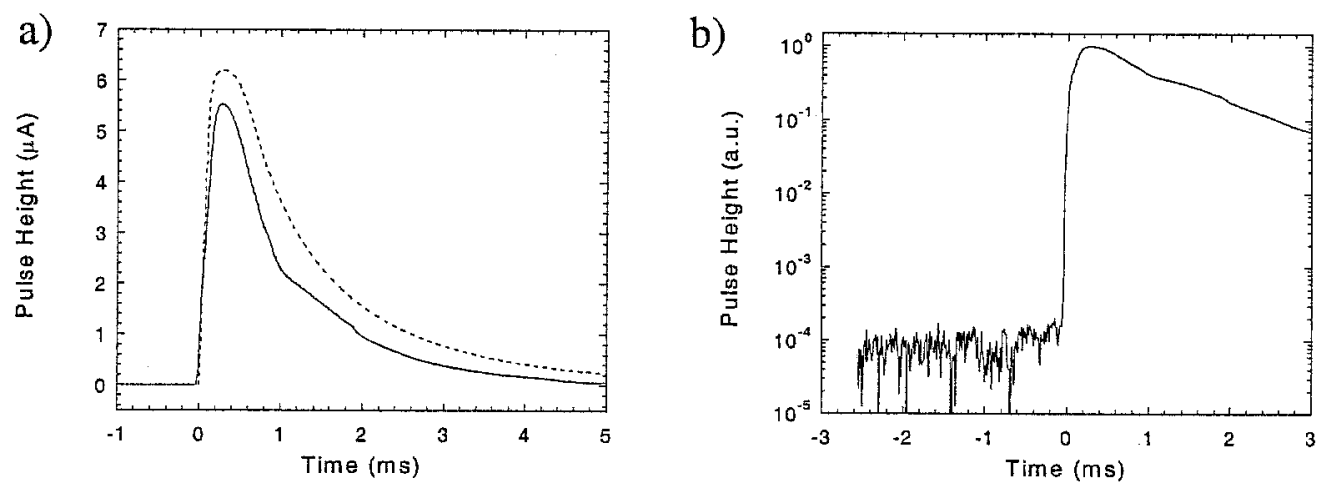

Fig. 7.24 SnTES\#8 measured pulse.

a) Solid line is measured pulse. Dotted line is modeled pulse. b) Normalized single pulse on logarithmic scale showing baseline noise. 


\begin{tabular}{|c|c|c|c|}
\hline & Parameter & Description & Value \\
\hline \multirow{5}{*}{ 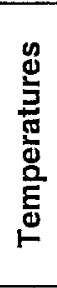 } & $\overline{T_{0}}$ & Operating & $95.2 \mathrm{mK}$ \\
\hline & $T_{T h}$ & Bias resistor & $-2 K$ \\
\hline & $T_{\text {Bath }}$ & Bath & $65 \mathrm{mk}$ \\
\hline & $T_{C}$ & Transition & $95.7 \mathrm{mK}$ \\
\hline & $\Delta T_{c}$ & Transition width & $2 \mathrm{mK}$ \\
\hline \multirow{4}{*}{$\begin{array}{l}0 \\
ه \\
0\end{array}$} & $C_{\text {Abs(eff })}$ & Effective absorber & $65 \mathrm{keV} / \mathrm{mK}$ \\
\hline & $C_{\text {TES(eff) }}$ & Effective TES & $25 \mathrm{keV} / \mathrm{mK}$ \\
\hline & $G_{S I N}$ & SiN membrane & $0.3 \mathrm{nW} / \mathrm{K}$ \\
\hline & $G_{A b s(e f f)}$ & Effective Stycast & $14 \mathrm{nW} / \mathrm{K}$ \\
\hline \multirow{4}{*}{ 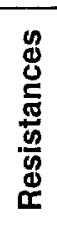 } & $R_{\text {Shunt }}$ & TES Shunt & $10 \mathrm{~m} \Omega$ \\
\hline & $R_{n}$ & TES (normal) & $453 \mathrm{~m} \Omega$ \\
\hline & $R_{0}$ & TES (operating) & $100.2 \mathrm{~m} \Omega$ \\
\hline & $R_{\text {lead }}$ & Lead & $8.3 \mathrm{~m} \Omega$ \\
\hline \multirow{4}{*}{$\stackrel{g}{\frac{\pi}{0}}$} & $V_{T h}$ & Bias voltage & $1.07 \mu \mathrm{V}$ \\
\hline & $l_{0}$ & Steady-state current & $9.5 \mu \mathrm{A}$ \\
\hline & $P_{0}$ & Bias power & $9.05 \mathrm{pW}$ \\
\hline & $\alpha$ & Transition "steepness" & $155-735$ \\
\hline \multirow{2}{*}{ 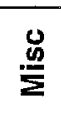 } & $E$ & Gamma ray energy & $8-60 \mathrm{keV}$ \\
\hline & $I_{\text {SQUID }}$ & SQUID noise & $10 \mathrm{pA} / \mathrm{Hz}^{1 / 2}$ \\
\hline
\end{tabular}

Table 7.5 SnTES\#8 values used for numerical model.

Fig. 7.25 shows the measured pulse heights and decay times compared with the numerical model. We see that the detector response to energy is close to linear, as expected when the changed in temperature and resistance of the TES is small. However, at lower energies, the modeled decay time deviates from data. Recall that we saw a similar effect in SnTES\#6. We attribute this to the decrease of $d R / d T$ near the bottom of the transition which is not adequately included in the analytic transition. 

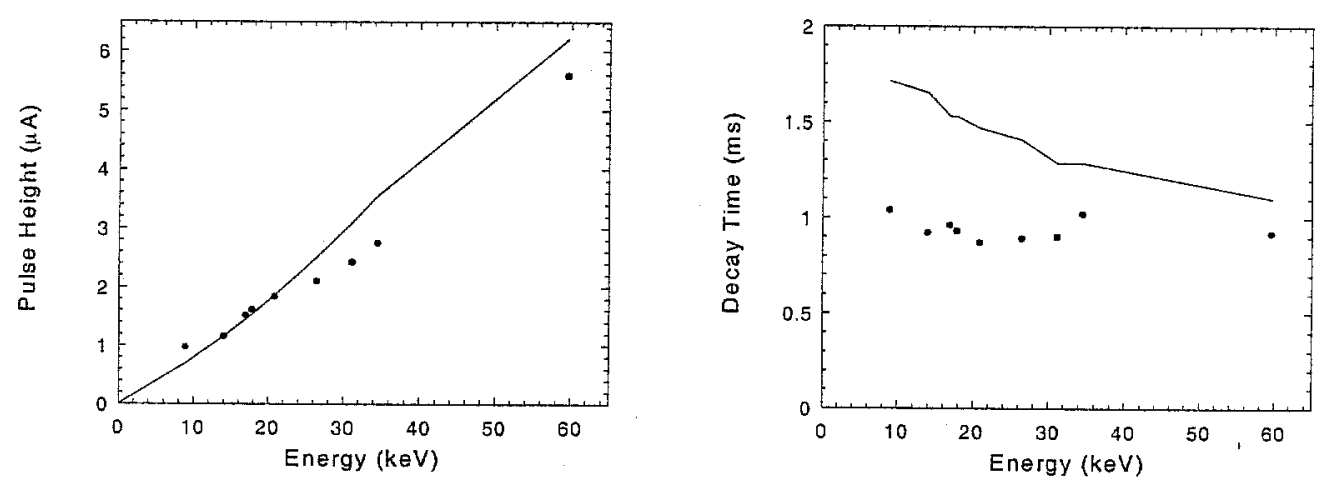

Fig. 7.25 SnTES\#8 pulse heights and decay times for measured (dots) and modeled (line) pulses.

From analysis of the pulse heights, we know that the largest change in resistance during a pulse is $170 \mathrm{~m} \Omega$, as shown in Fig. 7.26. Therefore, TES remained on the sharp part of the transition below $350 \mathrm{~m} \Omega$ at all times, satisfying the small TES temperature change requirement for linear response.

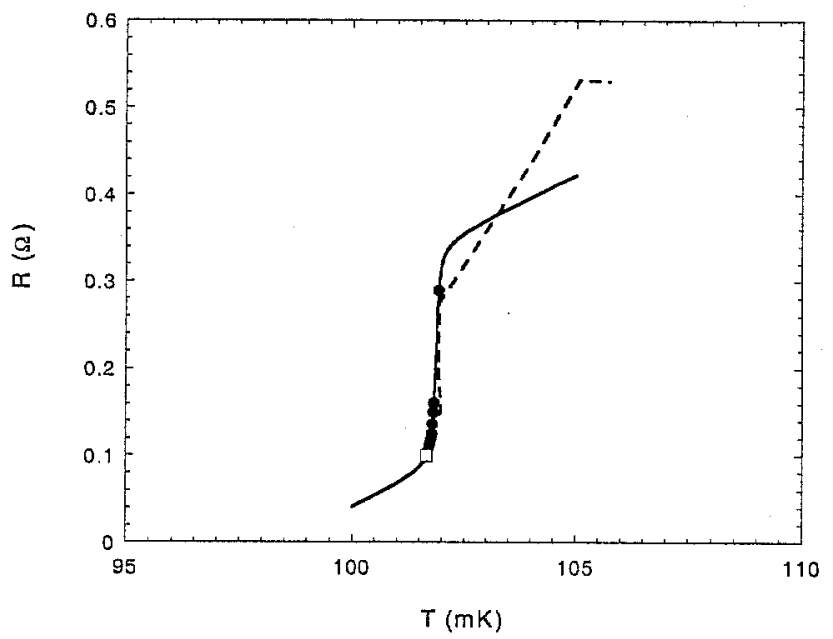

Fig. 7.26 Transition used in modeling SnTES\#8.

Solid line is the transition used in the numerical model. Dashed line is transition along constant $V_{B i a s}$ load line. The square indicates the bias point, and dots denote the trajectory maximum of different energies between $8 \mathrm{keV}$ and $60 \mathrm{keV}$. 


\subsubsection{Energy resolution and noise analysis}

Fig. 7.27a shows the ${ }^{241}$ Am spectrum measured by SnTES\#8. Fig. 7.27b shows the 60 $\mathrm{keV}$ peak fitted to a gaussian distribution. We measured an energy resolution of $87 \mathrm{eV}$ FWHM for $60 \mathrm{keV}$ gamma rays with analog low-pass filtering at the Nyquist frequency of $50 \mathrm{kHz}$. With digital optimal filtering, we measured $70 \mathrm{eV} \mathrm{FWHM}$ for $60 \mathrm{keV}$ gamma rays.
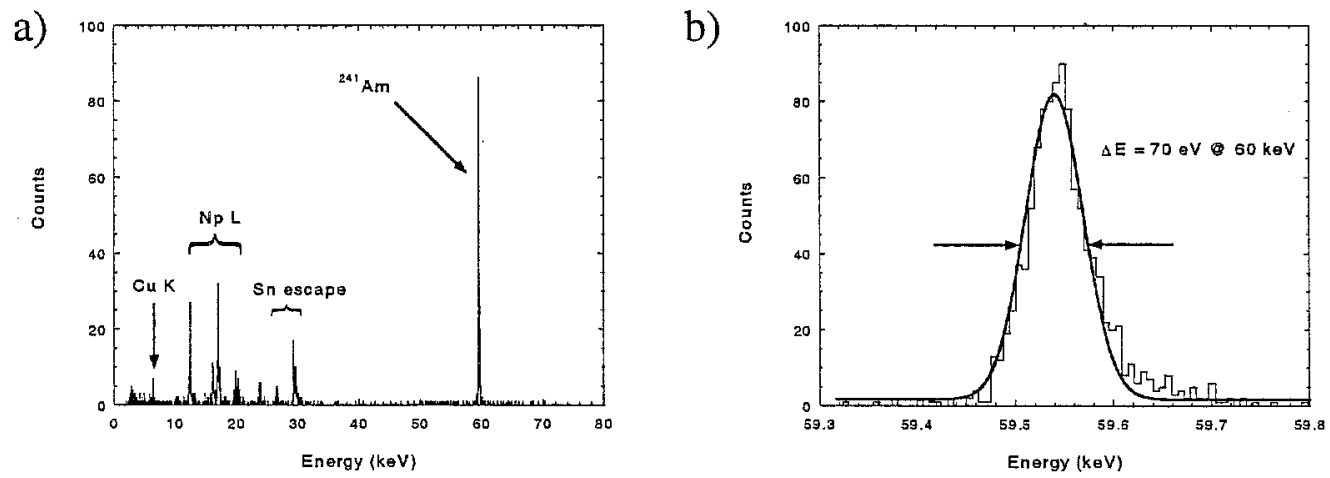

Fig. 7.27 SnTES\#8 spectrum

The measured noise spectrum for SnTES\#8 compared with theoretical noise sources is shown in Fig. 7.28. We see that the measured noise is significantly lower than SnTES\#6 and SnTES\#7. The $60 \mathrm{~Hz}$ pick-up is reduced, but still dominates at very low frequencies. Between $\sim 300 \mathrm{~Hz}$ and $\sim 3 \mathrm{kHz}$, the two-element phonon noise dominates. Above $\sim 3 \mathrm{kHz}$, Johnson noise from the TES shunt dominates, despite being reduced by operating the TES at a higher $R_{0}$. The signal pulse height is $5.59 \mu \mathrm{A}$. Again, Table 7.6 summarizes the integrated noise currents and the spectral linewidth contributions. 


\begin{tabular}{|c|c|c|}
\hline Noise Source & Current (rms) & Linewidth (FWHM) \\
\hline Phonon (2-element analytical) & $1.55 \mathrm{nA}$ & $39.1 \mathrm{eV}$ \\
\hline Johnson & $0.772 \mathrm{nA}$ & $19.5 \mathrm{eV}$ \\
\hline TES Shunt & $1.10 \mathrm{nA}$ & $27.7 \mathrm{eV}$ \\
\hline SQUID & $1.15 \mathrm{nA}$ & $29.0 \mathrm{eV}$ \\
\hline SQUID Shunt & $0.270 \mathrm{nA}$ & $6.80 \mathrm{eV}$ \\
\hline 60 Hz Pick-up & $2.42 \mathrm{nA}$ & $60.9 \mathrm{eV}$ \\
\hline Calculated Total & $3.39 \mathrm{nA}$ & $85.3 \mathrm{eV}$ \\
\hline Measured Total & $3.47 \mathrm{nA}$ & $87.5 \mathrm{eV}$ \\
\hline \multicolumn{2}{|c|}{ Table 7.6 SnTES\#8 noise contributions. }
\end{tabular}

The multi-element numerical simulation of phonon noise and Johnson noise yielded 2.74 $\mathrm{nA}$ and $0.218 \mathrm{nA}$, respectively. The numerical phonon noise is in poor agreement with the two-element analytical phonon noise. As in SnTES\#7, the integrated two-element analytical phonon noise is in good agreement with the measured noise. However, as with SnTES\#6, $60 \mathrm{~Hz}$ pick-up is the dominant noise source in this device.

The measured noise integrated from $100 \mathrm{~Hz}$ to $50 \mathrm{kHz}$ in quadrature is $3.47 \mathrm{nA}$, which is nearly a factor of three smaller than the measured noise in SnTES\#6 and SnTES\#7, giving us a signal-to-noise ratio of nearly 1610 which agrees with the low-pass filtered energy resolution of $87 \mathrm{eV}$ FWHM. 


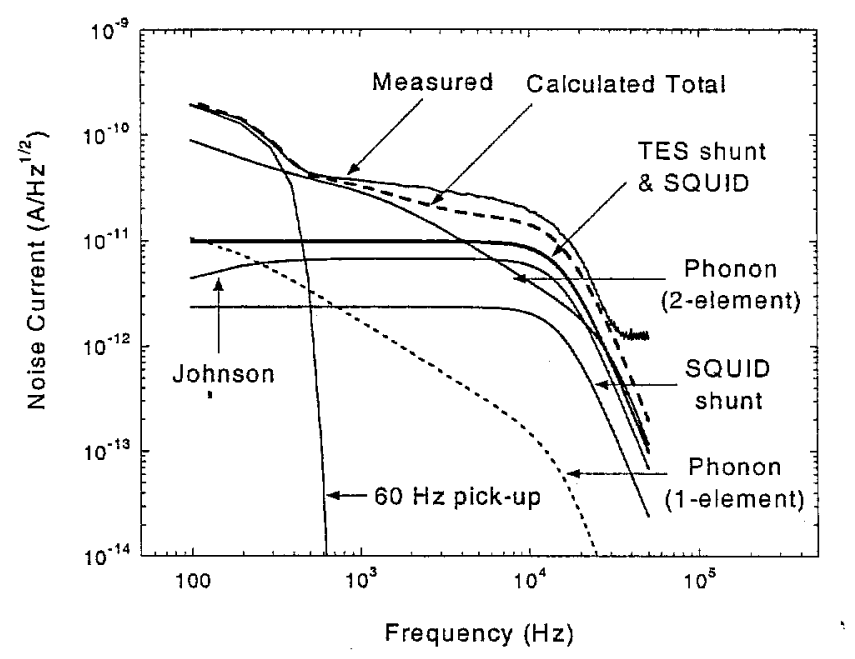

Fig. 7.28 SnTES\#8 Noise spectrum and calculated noise sources.

\subsection{Discussion and intercomparison}

The three devices described in this chapter vary in both geometry and performance. From our measurements, we see that the dominant noise at low frequency is $60 \mathrm{~Hz}$ pickup. The two-element phonon noise can, depending on conditions, dominate in the midrange frequencies. The intrinsic Johnson noise is significantly smaller. Therefore, the energy resolution of the detector is determined by external noise level and by the thermal couplings within the multi-element detector. The parameters for each device are shown in parallel along with the measured signal current and noise current in Table 7.7. Every one of these seventeen parameters contribute to the total performance of the detector. SnTES\#6 and SnTES\#7 have similar noise levels. SnTES\#7 has a much greater heat capacity than SnTES\#6 because of the higher $T_{0}$. Despite this, the signal size of SnTES\#7 is nearly three times greater than SnTES\#6 because SnTES\#7 has a larger $I_{0}$ resulting from a higher $T_{c}$ and a larger $\alpha$ from a sharper transition. SnTES\#7 and 
SnTES\#8 have similar $T_{0}$, but SnTES\#7 was operated at a lower $R_{0}$ (higher $\alpha$ ), giving a signal size twice that of SnTES\#8. But, it is the lower noise level due to higher $R_{0}$ and the more linear response of SnTES\#8 that yielded an energy resolution better than SnTES\#7 or SnTES\#6. We also see the contribution of $G_{A b s}$ to the phonon noise level. Eq. 3.50 states that the phonon noise magnitude is proportional to $G_{A b s}$, as evidenced by comparing $G_{A b s}$ between all three devices. At very high frequencies, we see some excess noise, which we attribute to rf pick-up. Without any alteration of the experimental setup, rf pick-up has been qualitatively observed to fluctuate by as much as a factor of four within a duration of several hours.

At the time of writing, our energy resolution of $70 \mathrm{eV} \mathrm{FWHM}$ at $60 \mathrm{keV}$ with a $\mathrm{Sn}$ absorber coupled to a TES is among the best measurements of its type. Other research groups in the field of low temperature detectors have also produced good results, but with smaller absorbers. Stahle, et al., [2] measured an energy resolution of $90 \mathrm{eV} \mathrm{FWHM} \mathrm{at}$ $32 \mathrm{keV}$ with a Sn absorber coupled to an ion-implantation doped Si thermistor. Silver, et al., [3] measured an energy resolution of $52 \mathrm{eV}$ FWHM at $60 \mathrm{keV}$ with a $\mathrm{Sn}$ absorber coupled to a neutron-transmutation-doped (NTD) Ge thermistor. Egelhof, et al., [4] measured an energy resolution of $75 \mathrm{eV}$ FWHM at $60 \mathrm{keV}$ with a $\mathrm{Sn}$ absorber coupled to a NTD Ge thermistor. Egelhof, et al., also used a $\mathrm{Pb}$ absorber and measured an energy resolution of $65 \mathrm{eV} \mathrm{FWHM}$ at $60 \mathrm{keV}$. Despite drastically different physical principles, we also include the magnetic calorimeter project in our comparison. Seidel, et al. measured and energy resolution of $120 \mathrm{eV}$ for hard x-rays up to $200 \mathrm{keV}$ [5] using an 
erbium-gold matrix as the absorber. Details regarding all of the above mentioned results are summarized in Table 7.8 .

Our microcalorimeters are unique in that they use TESs rather than semiconducting thermistors. Our high energy resolution is a significant achievement by virtue of the fact that our absorber is at least 40 times larger in volume than other detectors with comparable energy resolution.

\begin{tabular}{|c|c|c|c|c|}
\hline & Parameter & SnTES\#6 & SnTES\#7 & SnTES\#8 \\
\hline \multirow{5}{*}{ 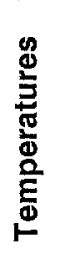 } & $T_{0}$ & $81.9-91.1 \mathrm{mK}$ & $99.7 \mathrm{mK}$ & $95.2 \mathrm{mK}$ \\
\hline & $T_{T h}$ & $-2 \mathrm{~K}$ & $-2 \mathrm{~K}$ & $-2 \mathrm{~K}$ \\
\hline & $T_{\text {Bath }}$ & $69.5-81.9 \mathrm{mK}$ & $65 \mathrm{mK}$ & $65 \mathrm{mK}$ \\
\hline & $T_{c}$ & $79.9 \mathrm{mK}$ & $98 \mathrm{mK}$ & $101.6 \mathrm{mK}$ \\
\hline & $\Delta T_{C}$ & $20.7 \mathrm{mK}$ & $4 \mathrm{mK}$ & $2 \mathrm{mK}$ \\
\hline \multirow{4}{*}{$\begin{array}{l}\text { ఠ } \\
\varnothing \\
0\end{array}$} & $C_{A b s(e f f)}$ & $1.54-1.94 \mathrm{keV} / \mathrm{mK}$ & $40 \mathrm{keV} / \mathrm{mK}$ & $65 \mathrm{keV} / \mathrm{mK}$ \\
\hline & $C_{T E S(\text { eff })}$ & $7.19-8.01 \mathrm{keV} / \mathrm{mK}$ & $10 \mathrm{keV} / \mathrm{mK}$ & $25 \mathrm{keV} / \mathrm{mK}$ \\
\hline & $G_{S i N}$ & $0.294 \mathrm{nW} / \mathrm{K}$ & $0.3 \mathrm{nW} / \mathrm{K}$ & $0.3 \mathrm{nW} / \mathrm{K}$ \\
\hline & $G_{A b s(\text { eff })}$ & $2.53-3.45 \mathrm{nW} / \mathrm{K}$ & $25 \mathrm{nW} / \mathrm{K}$ & $14 \mathrm{nW} / \mathrm{K}$ \\
\hline \multirow{4}{*}{ 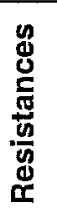 } & $R_{\text {Shunt }}$ & $20 \mathrm{~m} \Omega$ & $10 \mathrm{~m} \Omega$ & $10 \mathrm{~m} \Omega$ \\
\hline & $R_{n}$ & $360 \mathrm{~m} \Omega$ & $453 \mathrm{~m} \Omega$ & $453 \mathrm{~m} \Omega$ \\
\hline & $R_{0}$ & $34.3-195 \mathrm{~m} \Omega$ & $47.1 \mathrm{~m} \Omega$ & $100.2 \mathrm{~m} \Omega$ \\
\hline & $R_{\text {fead }}$ & $12.7 \mathrm{~m} \Omega$ & $8.44 \mathrm{~m} \Omega$ & $8.3 \mathrm{~m} \Omega$ \\
\hline \multirow{4}{*}{$\frac{\mathscr{g}}{\mathbf{m}}$} & $V_{T h}$ & $0.118-1.27 \mu \mathrm{V}$ & $0.861 \mu \mathrm{V}$ & $1.07 \mu \mathrm{V}$ \\
\hline & 10 & $1.63-10.2 \mu \mathrm{A}$ & $15.1 \mu \mathrm{A}$ & $9.5 \mu \mathrm{A}$ \\
\hline & $P_{0}$ & $0.105-6.10 \mathrm{pW}$ & $10.7 \mathrm{pW}$ & $9.05 \mathrm{pW}$ \\
\hline & $\alpha$ & $8.14-41.5$ & 250 & 735 \\
\hline \multirow{4}{*}{$\frac{\mathscr{O}}{\Sigma}$} & $E$ & $60 \mathrm{keV}$ & $8-60 \mathrm{keV}$ & $8-60 \mathrm{keV}$ \\
\hline & $I_{\text {Signal }}$ & $4.03 \mu \mathrm{A}$ & $10.73 \mu \mathrm{A}$ & $5.59 \mu \mathrm{A}$ \\
\hline & $I_{\text {Noise }}$ & $8.60 \mathrm{nA}$ & $8.80 \mathrm{nA}$ & $3.47 \mathrm{nA}$ \\
\hline & $\Delta E$ & $340 \mathrm{eV}$ & $240 \mathrm{eV}$ & $70 \mathrm{eV}$ \\
\hline
\end{tabular}

Table 7.7 Comparison of values used for numerical models. 


\begin{tabular}{|c|c|c|c|c|c|}
\hline Reference & Absorber & Volume & Sensor & $\Delta E$ & $\begin{array}{c}\text { Efficiency } \\
\text { at 60 keV }\end{array}$ \\
\hline Stahle [2] & $\mathrm{Sn}$ & $\begin{array}{c}0.2 \mathrm{~mm} \times 0.5 \mathrm{~mm} \\
\times 25 \mu \mathrm{m}\end{array}$ & $\mathrm{Si}$ & $\begin{array}{c}90 \mathrm{eV} @ \\
32 \mathrm{keV}\end{array}$ & $7.4 \%$ \\
\hline Silver [3] & $\mathrm{Sn}$ & $\begin{array}{c}0.5 \mathrm{~mm} \times 0.5 \mathrm{~mm} \\
\times 25 \mu \mathrm{m}\end{array}$ & NTD Ge & $\begin{array}{c}52 \mathrm{eV} \mathrm{@} \\
60 \mathrm{keV}\end{array}$ & $7.4 \%$ \\
\hline Egelhof [4] & $\mathrm{Sn}$ & $\begin{array}{c}0.3 \mathrm{~mm} \times 0.1 \mathrm{~mm} \\
\times 66 \mu \mathrm{m}\end{array}$ & NTD Ge & $\begin{array}{c}75 \mathrm{eV} @ \\
60 \mathrm{keV}\end{array}$ & $18 \%$ \\
\hline Egelhof [4] & $\mathrm{Pb}$ & $\begin{array}{c}0.3 \mathrm{~mm} \times 0.1 \mathrm{~mm} \\
\times 100 \mu \mathrm{m}\end{array}$ & NTD Ge & $\begin{array}{c}65 \mathrm{eV} @ \\
60 \mathrm{keV}\end{array}$ & $37 \%$ \\
\hline Seidel [5] & Er/Au & $\begin{array}{c}(\mathrm{Not} \mathrm{available,but} \\
\left.\mathrm{C}=10^{12} \mathrm{~J} / \mathrm{K}\right)\end{array}$ & Er/Au & $\begin{array}{c}120 \mathrm{eV} \mathrm{@} \\
200 \mathrm{keV}\end{array}$ & $\begin{array}{c}\text { (Not } \\
\text { available) }\end{array}$ \\
\hline Chow & $\mathrm{Sn}$ & $\begin{array}{c}1 \mathrm{~mm} \times 1 \mathrm{~mm} \\
\times 250 \mu \mathrm{m}\end{array}$ & TES & $\begin{array}{c}70 \mathrm{eV} \mathrm{@} \\
60 \mathrm{keV}\end{array}$ & $54 \%$ \\
\hline
\end{tabular}

Table 7.8 Comparison gamma-ray spectrometer results.

\subsection{Limitations}

As we saw with SnTES\#6 and SnTES\#7, the wide transition greatly affects the response of the detector. If the response had been linear, SnTES\#7 would have an energy resolution better than $120 \mathrm{eV}$. We have good control over the $T_{c}$ of the TES films. However, we have little understanding on controlling the width $\Delta T_{c}$ and shape of the transition. Furthermore, Stycast epoxy exerts stress on the film, potentially altering both $T_{c}, \Delta T_{c}$, and the shape of the transition. One remedy is to fabricate a "gluing pad" adjacent to the TES for attaching of the absorber without stressing the TES. This may be implemented in future devices.

As we saw with all three devices, $60 \mathrm{~Hz}$ pick-up is a significant noise problem. This is particularly important because of the millisecond-scale decay times of our microcalorimeters even after pulse shortening from electrothermal feedback. If the recovery time of the detector can be further shortened, the bandwidth can be extended into higher frequencies, allowing the $60 \mathrm{~Hz}$ pick-up to be removed by filtering. 
In the next chapter, we will operate the TES with an active bias which will linearize the response of the microcalorimeter and further reduce the decay time of the signals.

\section{References}

[1] W. Holmes, J.M. Gildemeister, P.L. Richards, Appl. Phys. Lett., 72, 2250'(1998).

[2] C. Stahle, Ph.D. Dissertation, Stanford University (1991).

[3] E. Silver, S. Bandler, H. Schnopper, S. Murray, M. Barbera, N. Madden, D. Landis, J. Beeman, E.E. Haller, Proc. SPIE, 4140, 397 (2000).

[4] A. Bleile, P. Egelhof, H.-J. Kluge, U. Liebisch, D. McCammon, H.J. Meier, O. Sebastián, C.K. Stahle, M. Weber, Nucl. Instr. Meth. A, 444, 488 (2000).

[5] C. Enss, A. Fleischmann, K. Horst, J. Schonefeld, J. Sollner, J.S. Adams, Y.H. Huang, Y.H. Kim, G.M. Seidel, Journ. Low Temp. Phys., 121, 137 (2000). 
Chapter 8

\section{ACtive Negative FeEdBaCK Bias}

\subsection{Motivation}

We saw in Chapter 7 that our microcalorimeters have demonstrated good results and great potential. However, they are vulnerable to $60 \mathrm{~Hz}$ pick-up and non-linear response to energy. We propose to operate our microcalorimeters in an active negative feedback bias mode. Active bias of TES microcalorimeters was first described by Nam [1] as a means to shorten pulses beyond the capability of electrothermal feedback. However, in our multi-element microcalorimeter, active bias has an additional benefit of linearizing the energy response.

\subsection{Theory}

Recall the differential equation for power flow in a TES given by Eq. 3.25. For strong voltage bias, we have $P_{\text {Bias }}=V_{0}^{2} / R(T)$ which yields an electrothermally shortened decay time given by Eq. 3.32. The time constant of the TES can be further shortened by actively reducing the bias voltage in proportion with the signal current such that the 
reduction in $P_{\text {Bias }}$ is greater than intrinsic electrothermal feedback. The voltage for active negative feedback bias is given by

Eq. 8.1

$$
V_{T E S}=V_{0}+R_{c} \cdot\left(I_{T E S}-I_{0}\right)
$$

where $V_{T E S}$ and $I_{T E S}$ are the voltage and current of the TES, respectively. $V_{0}$ and $I_{0}$ are the steady-state voltage and current of the TES, respectively. $R_{c}$ is a gain parameter relating the change in current through the TES with the active bias voltage. At equilibrium, $I_{T E S}=$ $I_{0}$, so $V_{T E S}=V_{0}$. During a negative current pulse, we have $I_{T E S}<I_{0}$ and subsequently $V_{T E S}$ $<V_{0}$ for positive $R_{c}$. The magnitude of the reduction of $V_{T E S}$ depends on the value of $R_{c}$. Eq. 8.1 can be combined with Ohm's law to give

Eq. 8.2

$$
I_{\text {TES }}=I_{0} \cdot\left(\frac{R_{c}-R_{0}}{R_{c}-R(T)}\right)
$$

We can estimate the active bias current signal by taking the Taylor expansion of Eq. 8.2 about $T_{0}+\Delta T$

Eq. 8.3

$$
I_{T E S}=I_{0}+I_{0} \frac{R_{0}}{R_{c}-R_{0}} \alpha \frac{\Delta T}{T_{0}}+\cdots
$$

where, as before, we identify the first term as the steady-state current and the second term as the first-order signal response to a small change in temperature. In the case of no active bias (voltage bias), we let $R_{c} \rightarrow 0$, thus recovering Eq. 3.24. In the case of 
extremely high-gain active bias, we have $R_{c} \approx R_{0}$. Eq. 8.2 may fail under these circumstances as it is an approximation which is valid for current signals smaller than $I_{0}$. Under high gain active bias, the current signal will "saturate" (or rather "bottom out") at a magnitude of $I_{0}$.

Substituting Eq. 8.2 into Eq. 3.25 gives us

Eq. 8.4

$$
C \frac{d T}{d t}=\left(\frac{R_{c}-R_{0}}{R_{c}-R(T)}\right)^{2} I_{0}^{2} R(T)-K \cdot\left(T^{N}-T_{B a t h}^{N}\right)
$$

The time constant for this differential equation is

Eq. 8.5

$$
\tau_{\text {Active }}=\frac{\tau_{0}}{1+\frac{\alpha}{N}\left(1-\frac{T_{B a h h}^{N}}{T_{0}^{N}}\right)\left(\frac{R_{0}+R_{c}}{R_{0}-R_{c}}\right)}
$$

For $0<R_{c}<R_{0}$, the active bias decay time $\tau_{\text {Active }}$ is shorter than the electrothermal feedback decay time given in Eq. 3.32.

In our multi-element microcalorimeter, negative feedback also decreases $\Delta T_{T E S}$, the change in temperature of the TES. For a microcalorimeter in the absence of feedback and weakly coupled to the cold bath, $\Delta T_{T E S}=E / C_{\text {Total }}$. With electrothermal feedback, the reduction in $P_{\text {Bias }}$ shortens the decay time as well as decreases $\Delta T_{T E S}<E / C_{\text {Total }}$. For our microcalorimeter design, if the energy flow rate from the absorber to the TES is less than 
$P_{\text {Bias }}$ of the TES, then strong active negative feedback bias (with $R_{c} \approx R_{0}$ ) can reduce $P_{\text {Bias }}$ sufficiently that $\Delta T_{T E S} \ll E / C_{\text {Total }}$ is decreased by orders of magnitude, thus effectively maintaining the TES at constant temperature during a pulse. A TES that operates at near constant temperature (and near constant resistance) during a pulse becomes impervious to any non-linear features on the transition, thereby guaranteeing a linear response to energy. Even though the TES does not change in temperature or resistance, the signal exists in the $P_{\text {Bias }}$ profile, which is proportional to both $I_{T E S}$ and $V_{T E S}$.

\subsection{Model}

\subsubsection{Pulse shortening \& reduced $\Delta T_{T E S}$}

We apply active bias to the 2-element microcalorimeter model. Fig. 8.1a shows a simulation of the absorber and TES temperature profiles after absorption of a gamma-ray in the voltage bias mode. We see that the TES temperature rises to nearly the absorber temperature, then the absorber and TES exponentially cool together. This decay time is limited by the total heat capacity and the thermal conductivity of the weak link to the cold bath. Fig. 8.1b shows a simulation of the absorber and TES temperature profiles in the active bias mode. We see that the active bias maintains the TES at near-constant temperature. This results in a larger temperature difference between the absorber and the TES, allowing the absorber to cool off faster. This decay time is limited by the absorber heat capacity and the thermal conductivity of the coupling between the absorber and the TES. These calculations simulate the near-constant TES temperature operation and the resultant additional pulse shortening. 

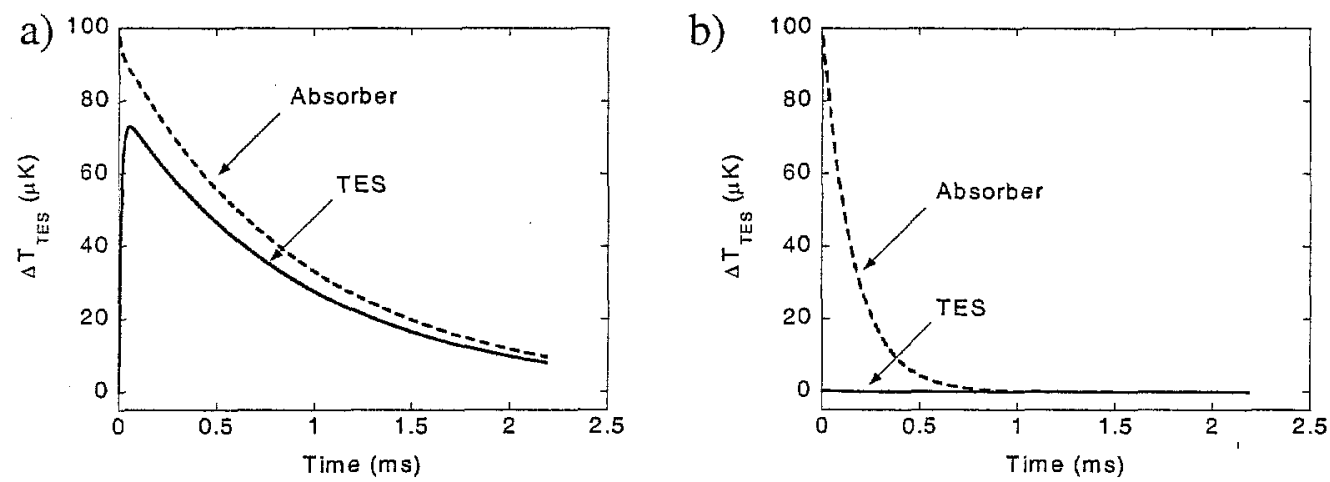

Fig. 8.1 Simulation of absorber and TES temperatures. a) voltage bias mode, b) active bias mode.

\subsubsection{Linear response}

We can simulate the extreme case of a non-linear superconducting transition and observe the detector response to energy [2]. Fig. 8.2a shows a transition with a discontinuity at $0.5 \Omega$. We see that low energies that traverse the linear part of the transition have a linear response. However, higher energies that cross over the "kink" have a different response from the low energy signals, thus resulting in an overall non-linear response to energy (Fig. $8.2 \mathrm{~b}$ ). Fig. $8.3 \mathrm{a}$ shows the same transition operated under active bias. The TES maintained a near-constant temperature for all energies such that the points in the plot indicating the changes in temperature are unresolved. The resulting current pulse heights are linear with energy. Fig. 8.4 shows the simulated TES change in temperature for different energies. We see that, as described, earlier, $\Delta T_{T E S}$ in the active bias mode is much less than the case in the voltage bias mode, which is less than the case in the absence of any bias. 

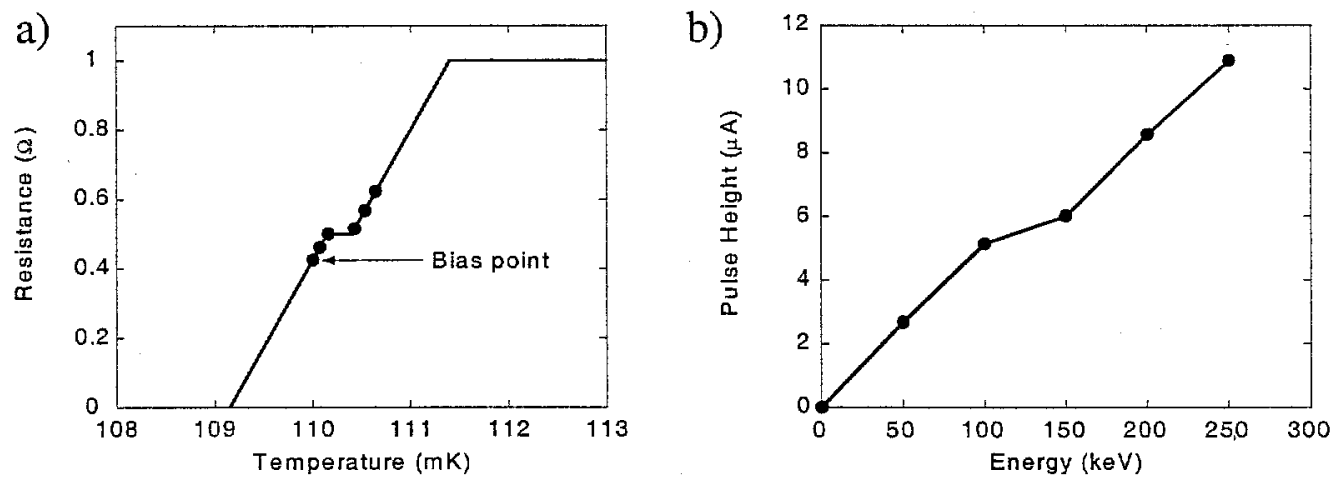

Fig. 8.2 Simulation of non-linear transition and energy response in voltage bias mode. a) Dots indicate the maximum temperature and resistance attained by pulses ranging in energy from 0 to $250 \mathrm{keV}$. The zero energy case shows the bias point. b) Current signal pulse heights for different energies.
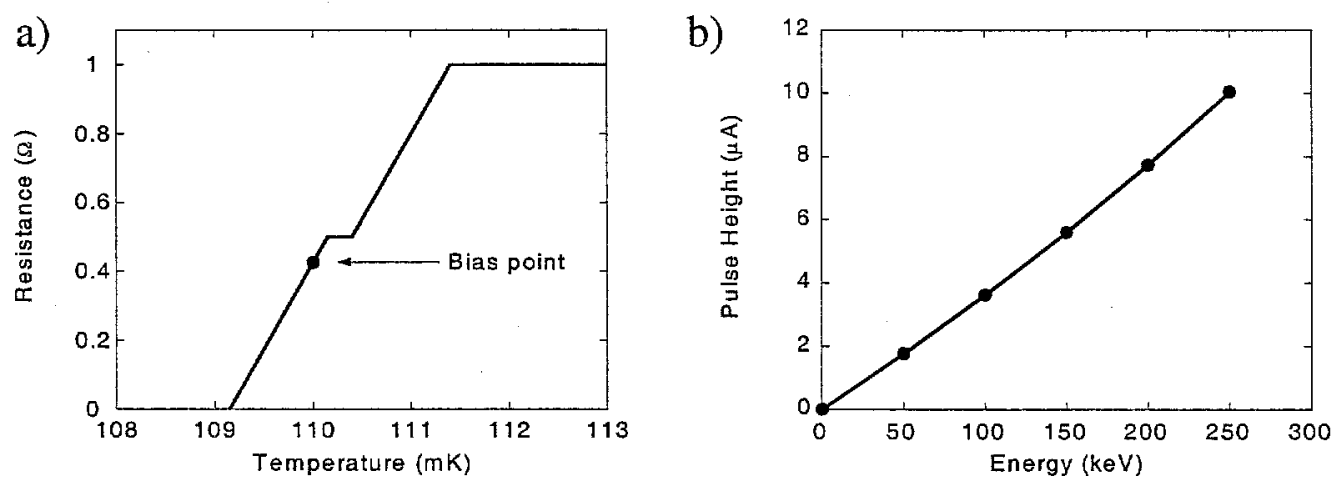

Fig. 8.3 Simulation of non-linear transition and energy response in active bias mode. a) The change in temperature of the TES from different energies is so small that the points cannot be resolved. b) Current signal pulse heights for different energies is linear. 


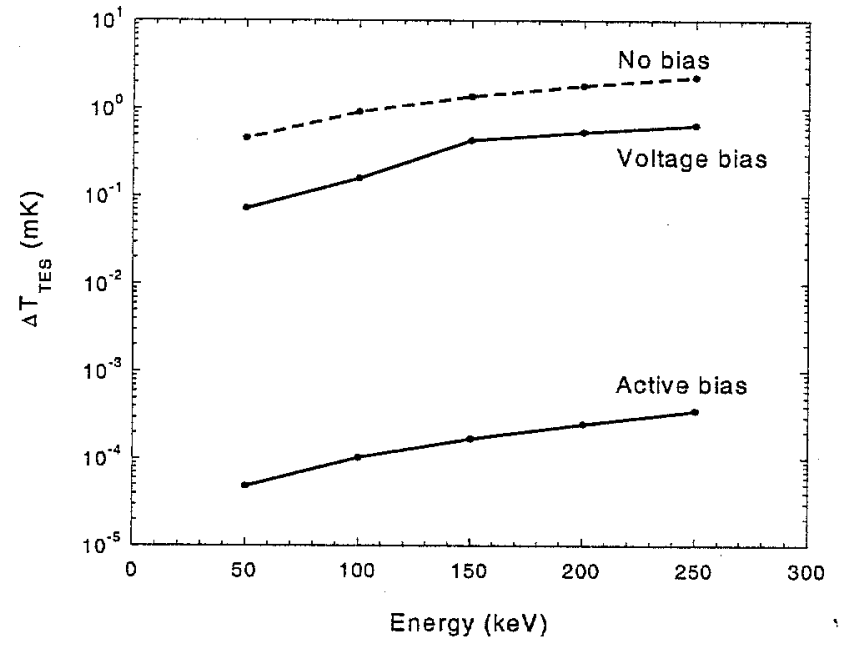

Fig. 8.4 Simulation of reduction in $\Delta T_{T E S}$ from voltage bias mode and active bias mode.

\subsection{Noise}

We can derive the expression for current noise with active feedback by starting with the differential equation with noise power terms,

Eq. 8.6

$$
C \frac{d T}{d t}=\left(\frac{R_{c}-R_{0}}{R_{c}-R(T)}\right)^{2} I_{0}^{2} R(T)-K \cdot\left(T^{N}-T_{B a t h}^{N}\right)+P_{J}(t)+P_{P h}(t),
$$

where $P_{J}(t)=I_{0} V_{J}(t)$ is the TES Johnson noise term and $P_{P h}(t)$ is the phonon noise term. Following our method of derivation in Section 3.5 (page 52), we find expressions for TES Johnson noise and phonon noise currents, 
Eq. 8.7

$$
I_{J}(\omega)=V_{J}(\omega)\left(\frac{1}{R_{0}}-\frac{I_{0}^{2} \alpha}{i \omega C T_{0}-P_{0} \alpha\left(1+\frac{2 R_{0}}{R_{c}-R_{0}}\right)+G T_{0}}\right)
$$

Eq. 8.8

$$
I_{P h}(\omega)=\frac{P_{P h}(\omega) I_{0} \alpha}{i \omega C T_{0}-P_{0} \alpha\left(1+\frac{2 R_{0}}{R_{c}-R_{0}}\right)+G T_{0}}
$$

Fig. 8.5 shows the theoretical noise spectra using TES parameters given in Table 3.1 and varying values of $R_{c}$. In the case of no active bias (constant voltage bias only), we let $R_{c}$ $\rightarrow 0$. The noise expressions become identical to Eq. 3.38 and Eq. 3.39 for $R_{T h} \rightarrow 0$. In the case of very strong active feedback bias, we let $R_{c} \rightarrow R_{0}$. The phonon noise level becomes very small as the bandwidth is increased due to additional pulse shortening. The TES Johnson noise approaches the white noise value. Note that the case of $R_{c}=0$ in Fig. 8.5 differs from Fig. 3.4 (page 57) due to inclusion of non-zero $R_{T h}$ in the expressions plotted in Fig. 3.4.

From Section 3.5 (page 52) we know that with a strong voltage bias, Johnson noise creates current fluctuations that feedback to change the temperature such that, at low frequencies, the change in current is compensated for by the temperature change. Thus, the measured current noise is reduced at the output. However, with strong active bias the circuit holds the temperature fixed, such that the system can no longer cancel out the current fluctuations induced by Johnson noise. Thus, active feedback does not produce the suppression of Johnson noise at low frequencies observed in the constant voltage case. While the shape and magnitude of the noise changes, the total signal-to-noise ratio, 
and therefore the resolution for the simple calorimeter is the same with active feedback as it is for the constant voltage bias case [1].

a)

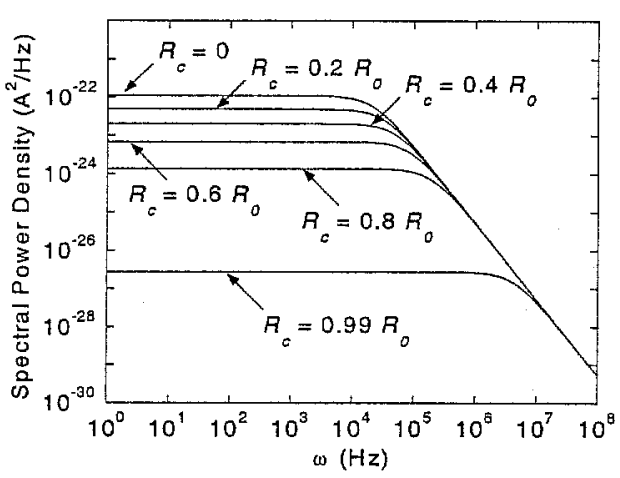

b)

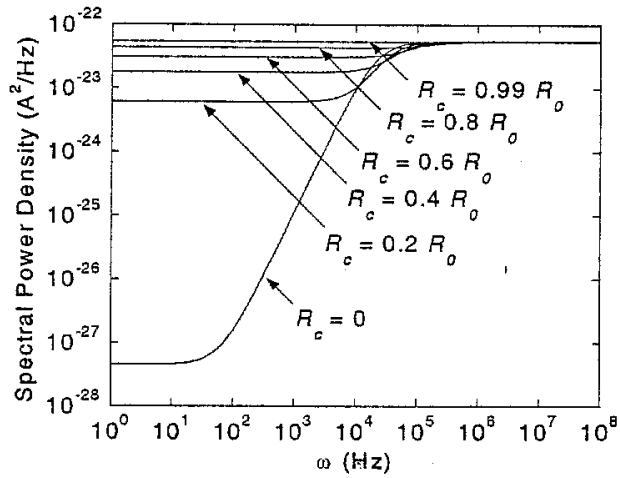

Fig. 8.5 a) Theoretical phonon noise spectra and b) Johnson noise spectra for varying values of $R_{c}$.

\subsection{Circuit}

Active bias requires that we amplify the negative current pulse signal and feed it back to the bias voltage via a feedback resistor. During a pulse, the bias voltage is then actively reduced in proportion to the signal current. Fig. 8.6 shows a summing circuit that is appropriate for active bias [3]. This circuit was chosen because the output of this circuit is given by

Eq. 8.9

$$
V_{\text {out }}=-\left(V_{1}+V_{2} \frac{R_{1}}{R_{2}}\right)
$$

which is similar in form to Eq. 8.1. The complete active bias circuit is shown in Fig. 8.7. The right half of the circuit is the original low-impedance TES voltage bias circuit. Steady-state bias voltage is applied at "V $\mathrm{Bias}_{0}$ ". The bias voltage can be measured at "V $\mathrm{V}_{\mathrm{Bias}}$ monitor". The negative current pulses are measured " $\mathrm{V}_{\mathrm{FB}}$ ". The current signal is 
ac coupled to remove the steady-state $I_{0}$ offset. Occasionally, the SQUID inductors may be wired in reverse such that the signals are inverted. We can explicitly correct the polarity of the signal in this circuit. The gain of the active feedback bias is controlled by the variable resistor $R_{\text {Gain }}$. The negative signal is summed with the steady-state bias voltage to reduce the bias voltage, completing our active negative feedback loop. The bias voltage applied at " $\mathrm{V}_{\text {Bias }}$ monitor" with this circuit is given by

Eq. 8.10

$$
V_{b i a s}=V_{b i a s 0}+\frac{R_{i n}}{R_{G a i n}}\left(V_{F B}-V_{F B 0}\right),
$$

where $V_{F B O}$ is the SQUID output for $I_{0}$. Using the circuit analysis given in Section 6.2.2 (page 118), we rewrite Eq. 8.10 in terms of $V_{T E S}$ and $I_{T E S}$ and get,

Eq. 8.11

$$
V_{T E S}=V_{0}+\left(B \frac{R_{i n} R_{F B}}{R_{\text {Gain }} R_{\text {Bias }}}-1\right) R_{\text {Shunt }}\left(I_{T E S}-I_{0}\right)
$$

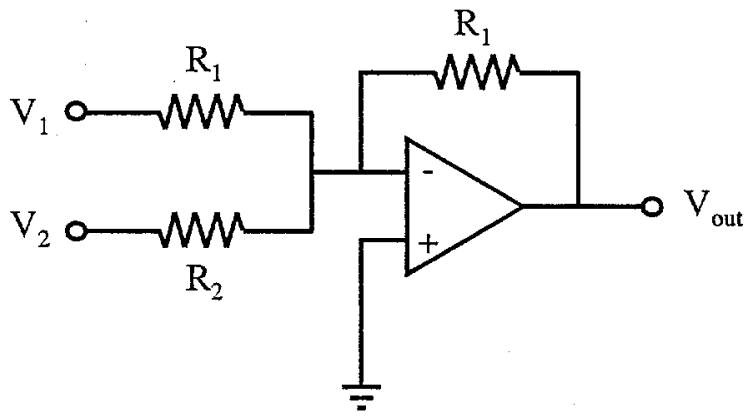

Fig. 8.6 Summing element of active bias circuit. 


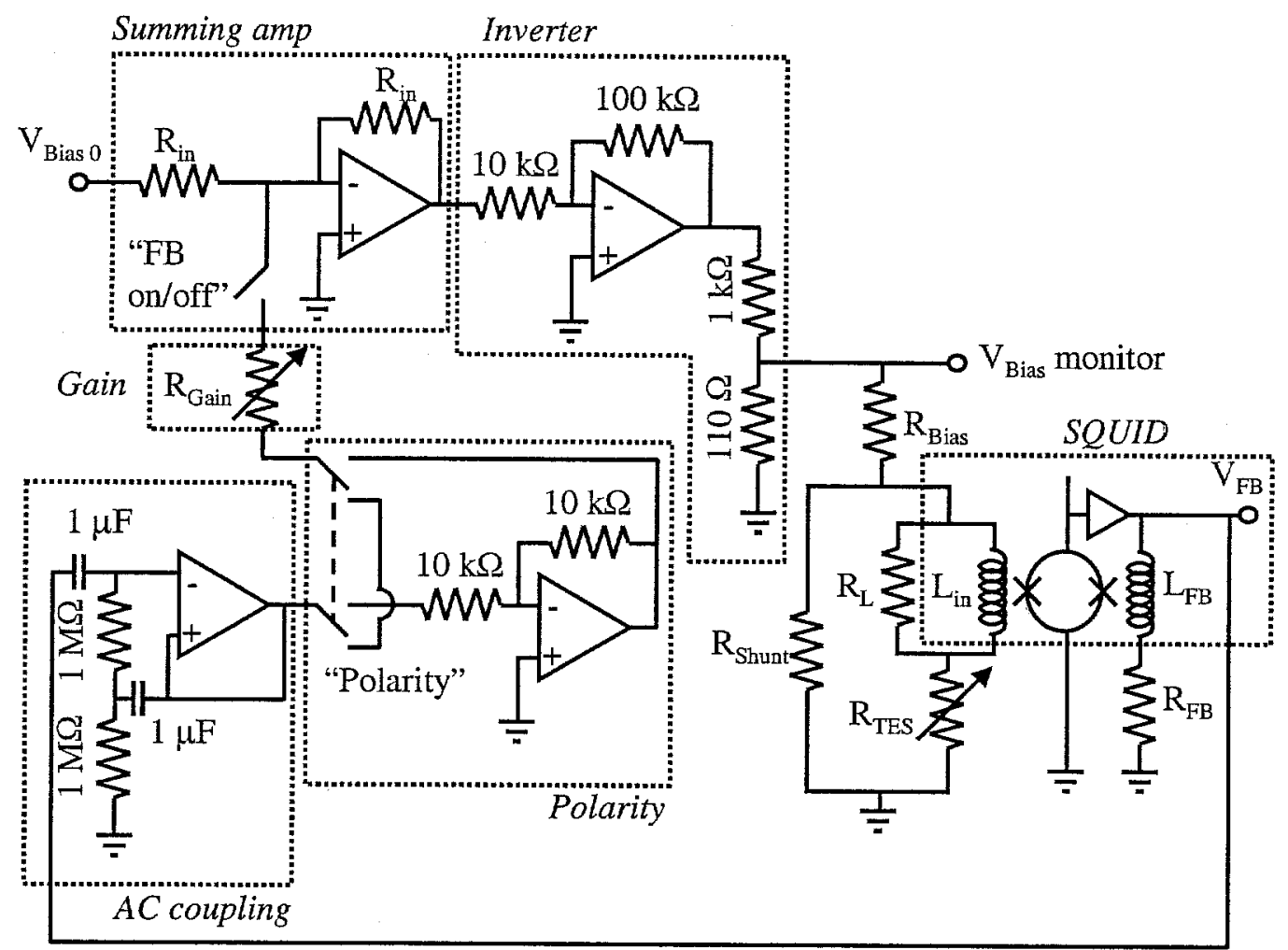

Fig. 8.7 Complete active negative feedback bias circuit.

Recall that $B$ is the ratio of the SQUID input coil and feedback coil inductances, which ranges from 8 to 15 for our SQUIDs (Section 6.2.2, page 118). Comparing this with Eq. 8.1 , we identify the feedback gain parameter as

Eq. 8.12

$$
R_{c}=\left(B \frac{R_{i n} R_{F B}}{R_{\text {Gain }} R_{\text {Bias }}}-1\right) R_{\text {Shunt }}
$$

Note that the value of $R_{\text {Gain }}$ is inversely proportional with the feedback gain. 


\subsection{SnTES\#7 revisited}

We recall in Section 7.8.1 (page 148) that the energy response of device SnTES\#7 is nonlinear due to large changes in TES temperature during a pulse. Therefore, this an ideal candidate for operating in the active negative feedback bias mode.

We operated SnTES\#7 in low-gain and high-gain active bias modes and compared with data acquired using constant voltage bias mode at the same bias point [4]. The difference between low-gain and high-gain active bias modes is in the value of $R_{c}$. In these experiments, low gain corresponds to $R_{c} \approx 0.5 R_{0}$ and high gain corresponds to $R_{c} \approx$ $0.85 R_{0}$. The effects of active bias such as pulse shortening and near-constant TES temperature exists in both high gain and low gain modes, but are more pronounced with high-gain than with low gain. For low-gain active bias, $R_{\text {Gain }}=21.5 \mathrm{k} \Omega$. For high-gain active bias, $R_{\text {Gain }}=10.58 \mathrm{k} \Omega$. The value of $R_{\text {in }}=10 \mathrm{k} \Omega$. All other resistors have the same value as in Section 7.8 (page 147)

\subsubsection{Pulse shortening}

The natural time constant of SnTES\#7 is $26.6 \mathrm{~ms}$. Fig. 8.8 shows the decay times of SnTES\#7 when operated in different bias modes for many different energies. Voltage biased electrothermal feedback shortened the decay time to $\sim 0.7 \mathrm{~ms}$. Application of lowgain active bias further shortened the decay time to $\sim 0.4 \mathrm{~ms}$. High-gain active bias shortened the decay time to $\sim 0.2 \mathrm{~ms}$, which is limited by the rate at which heat flows out

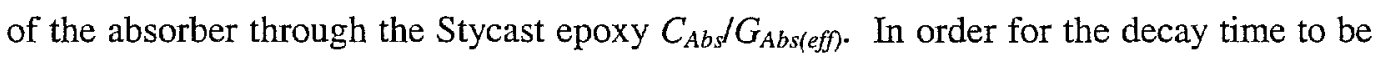
further shortened, the design of the microcalorimeter would have to be changed. 


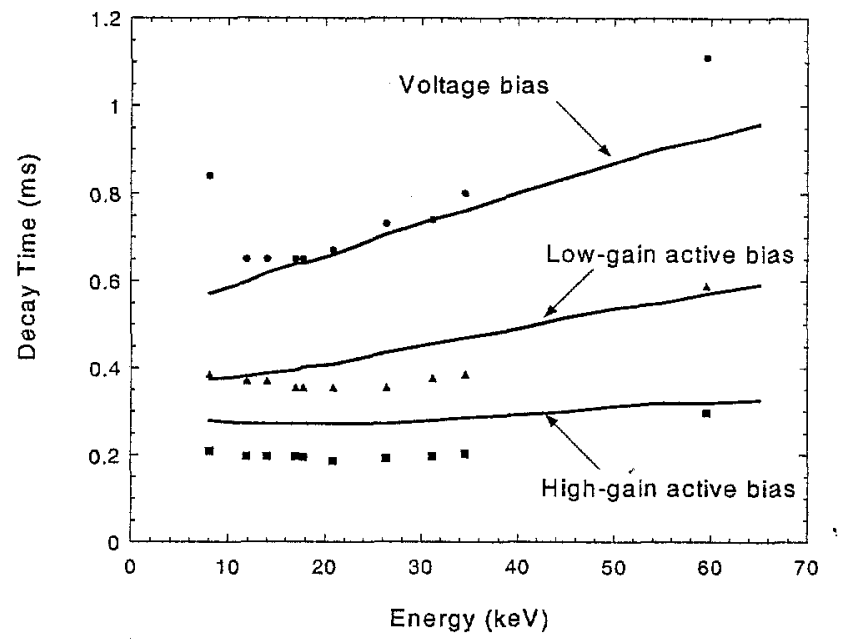

Fig. 8.8 SnTES\#7 decay times with different bias modes.

Dots are voltage biased data, triangles are low-gain active biased data $\left(R_{c} \approx 0.5 R_{0}\right)$, squares are high-gain active biased data $\left(R_{c} \approx 0.85 R_{0}\right)$, lines are numerical models

\subsubsection{Reduced temperature excursion}

From the IV characteristics, we were able to derived the superconducting transition of

SnTES\#7. The bias point and pulses allow us to calculate the change in resistance during a pulse. Thus, we can infer the TES change in temperature of the pulses. Fig. 8.9 shows the TES change in temperature for a range of energies. At low energies, $\Delta T_{T E S}$ under high-gain active bias is approximately a factor of three smaller than under voltage bias. At high energies, the absorber-to-TES energy transfer rate is greater than $P_{\text {Bias }}$ and active bias is inefficient at reducing $\Delta T_{T E S}$. 


\subsubsection{Linearization of spectrum}

Fig. 8.10 shows the pulse heights of SnTES\#7 at different energies and bias modes. In the voltage bias mode, the non-linear response of the TES is due to large temperature excursions. With low-gain active bias, the response is still non-linear. With high-gain active bias, the low-energy response is much closer to linear, but the high-energy response remains non-linear due to pulse saturation. Fig. 8.11 shows $60 \mathrm{keV}$ pulses measured with voltage bias and high-gain active bias. We see that under high-gain active bias, the pulse is saturated because the energy arrival rate is significantly greater than $P_{\text {Bias. }}$. However, at low energies, high-gain active bias is able to linearize the energy response of SnTES\#7. Fig. 8.12 shows residuals from a linear fit for all three bias modes. We see that high-gain active bias has the smallest deviation from a linear response to energy.

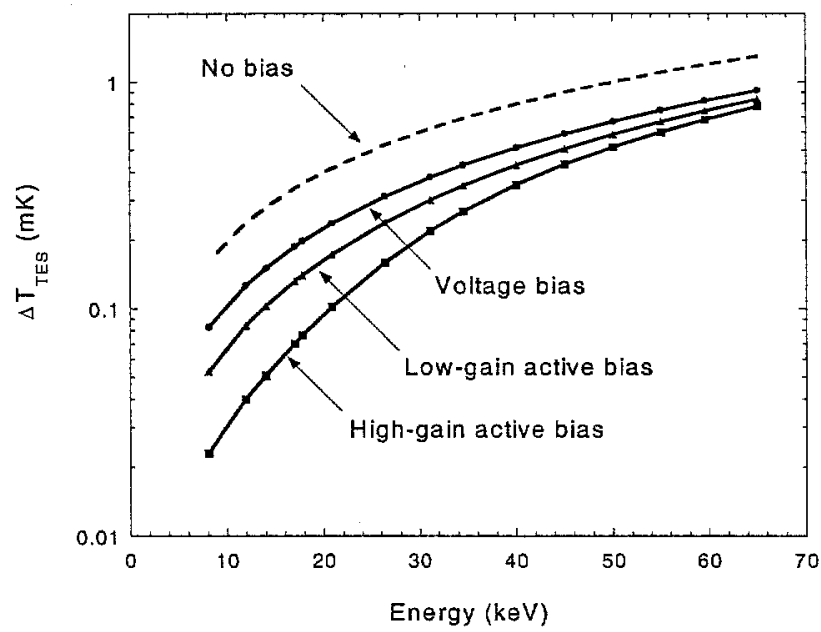

Fig. 8.9 SnTES\#7 TES change in TES temperature with different bias modes.

Dots are voltage biased data, triangles are low-gain active biased data $\left(R_{c} \approx 0.5 R_{0}\right)$, squares are high-gain active biased data $\left(R_{c} \approx 0.85 R_{0}\right)$, lines are numerical models. Dashed line is the temperature rise equal to the photon energy divided by the total heat capacity of the microcalorimeter. 


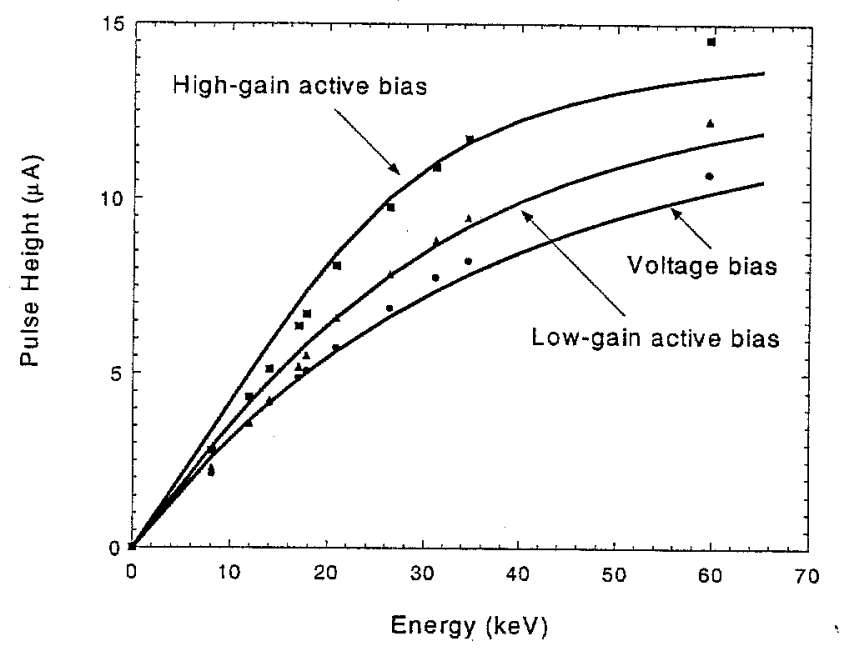

Fig. 8.10 SnTES\#7 pulse heights with different bias modes.

Dots are voltage-biased data, triangles are low-gain active-biased data $\left(R_{\mathrm{c}} \approx 0.5 R_{0}\right)$, squares are high-gain active-biased data $\left(R_{c} \approx 0.85 R_{0}\right)$, solid lines are numerical models
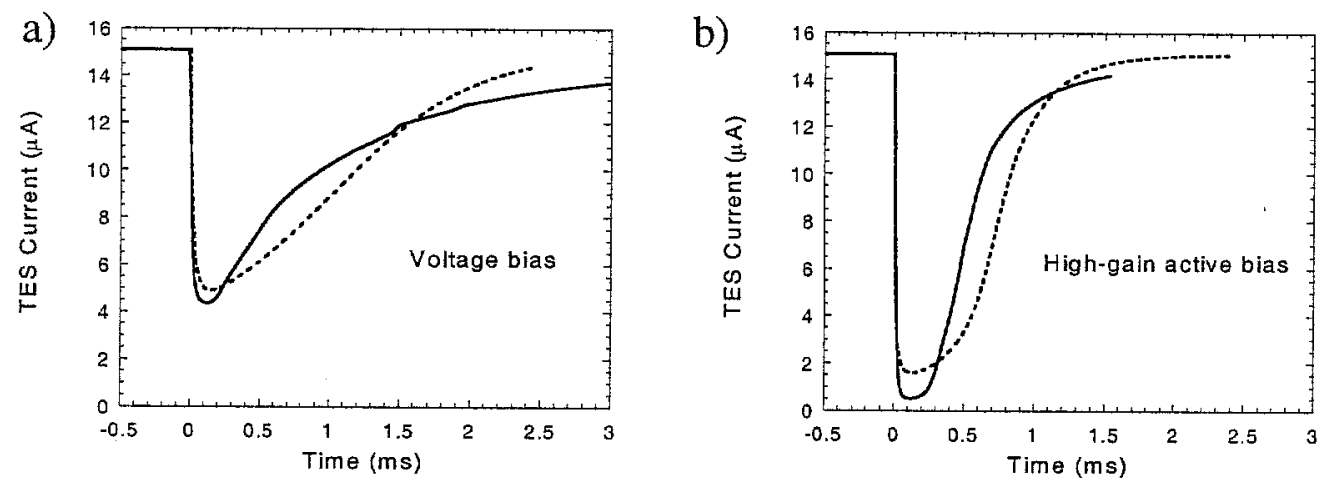

Fig. 8.11 SnTES\#7 $60 \mathrm{keV}$ pulse shapes with different bias modes. Solid lines are data, dotted lines are numerical models. 


\subsubsection{Energy resolution}

Fig. 8.13 shows the low-energy spectral response of SnTES\#7 operated with different bias modes. After compensating for non-linearity in the voltage bias and low-gain active bias cases, all three spectra exhibit the same energy resolution of $120 \mathrm{eV}$ for $17.74 \mathrm{keV} \mathrm{x}$ rays. We see significant peak shifting for the $21 \mathrm{keV}$ spectral line near the pulse height of 1.2 a.u., indicating the onset of non-linear behavior. The unchanged energy resolution is encouraging, as it suggests that our active negative feedback loop did not increase the amount of $60 \mathrm{~Hz}$ and if pick-up.

For the microcalorimeter to have a linear response at high energy, we must increase $P_{\text {Bias }}$. With a fixed cryostat base temperature, the applied bias power can be increased by increasing $T_{c}$ and/or $G_{S i N}$. We know that changing either $T_{c}$ or $G_{S i N}$ will affect the pulse height and signal-to-noise ratio, thus requiring significant redesign of the microcalorimeter. 


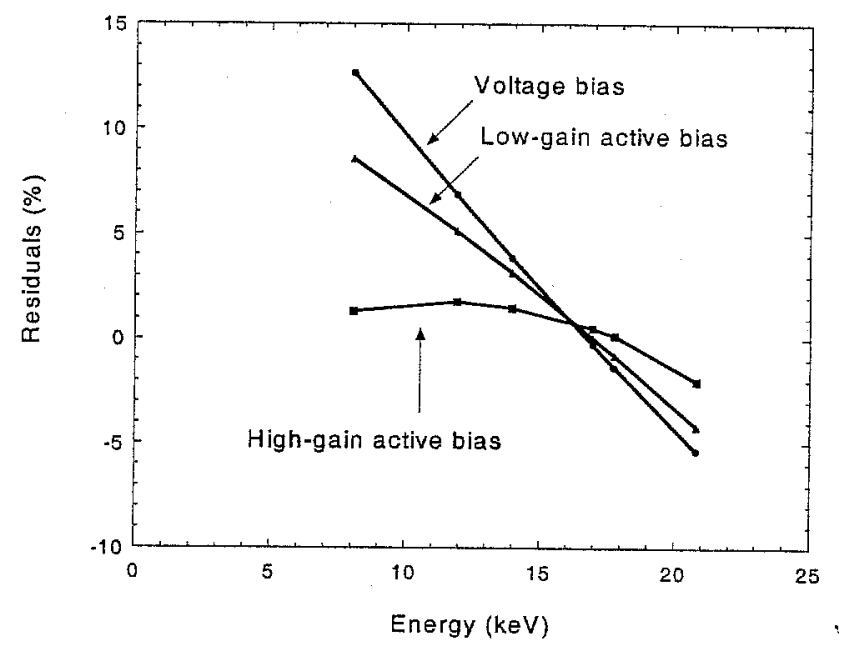

Fig. 8.12 SnTES\#7 spectrum linearity residuals.

Dots are voltage bias, triangles are low-gain active bias data $\left(R_{c} \approx 0.5 R_{0}\right)$, squares are high-gain active bias data $\left(R_{c} \approx 0.85 R_{0}\right)$.

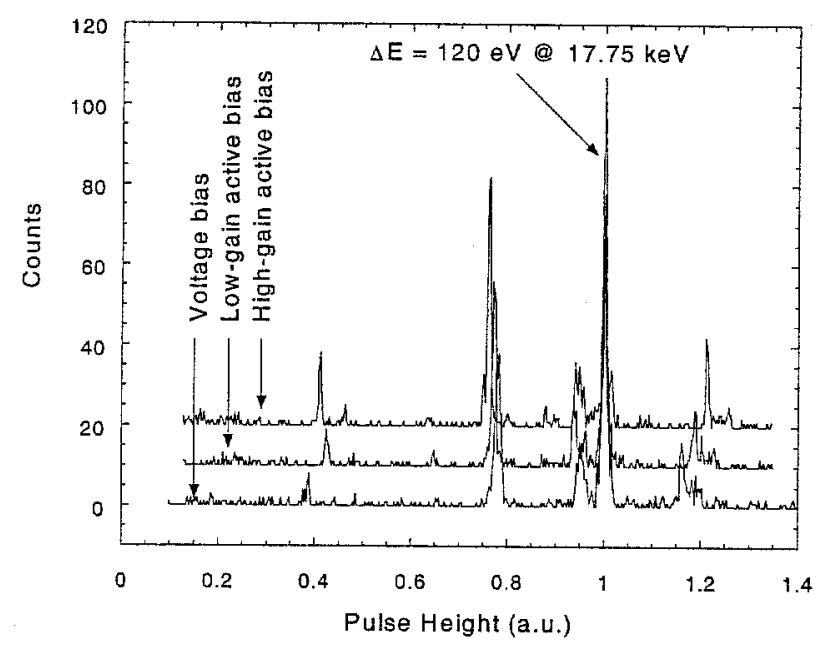

Fig. 8.13 SnTES\#7 spectra measured with different bias modes. 


\section{References}

[1] S.W. Nam, B. Cabrera, P. Colling, R.M. Clarke, E. Figueroa-Feliciano, A.J. Miller, R.W. Romani, IEEE Trans. on Appl. Supercond., 9, 4209 (1999).

[2] D.T. Chow, A. Loshak, M.L. van den Berg, M. Frank, T.W. Barbee, Jr., S.E. Labov, Proc. SPIE, 4141, 488 (2000).

[3] P. Horowitz, W. Hill, The Art of Electronics, Cambridge (1989).

[4] D.T. Chow, M.L. van den Berg, A. Loshak, M. Frank. T.W, Barbee, Jr., S.E. Labov, IEEE Trans. on Appl. Supercond., 11, 743 (2001). 


\section{Chapter 9}

\section{SuMMARY AND CONCLUSIONS}

We have demonstrated that a bulk absorber coupled to a TES can serve as a good gamma-ray spectrometer. Our measured energy resolution of $70 \mathrm{eV}$ at $60 \mathrm{keV}$ is among the best measurements in this field. We have also shown excellent agreement between the noise predictions and measured noise. Despite this good result, we noted that our detector design has shortcomings with a low count rate and vulnerabilities with the linearity of energy response. We addressed these issues by implementation of an active negative feedback bias. We demonstrated the effects of active bias such as additional pulse shortening, reduction of TES change in temperature during a pulse, and linearization of energy response at low energy. Linearization at higher energy is possible with optimized heat capacities and thermal conductivities of the microcalorimeter. However, the current fabrication process has low control and repeatability over the thermal properties. Thus, optimization of the detector performance is difficult until the fabrication process is improved. Currently, several efforts are underway to better control the fabrication of our gamma-ray spectrometers. We are developing a full-wafer process to produce TES films. We are investigating the thermal conductivity and surface 
roughness of thicker $\mathrm{SiN}$ membranes. We are exploring alternative methods to couple the absorber to the TES film for reproducibility. We are also optimizing the thermal conductivities within the detector to minimize two-element phonon noise. We are experimenting with different absorber materials to optimize absorption efficiency and heat capacity. We are also working on minimizing Johnson noise from the TES shunt and SQUID amplifier noise. We have shown that our performance, noise, and active bias models agree very well with measured data from several microcalorimeters. Once the fabrication improvements have been implemented, we have no doubt that our gamma-ray spectrometer will achieve even more spectacular results. 
Appendix A.

\section{FABRICATION PROCEDURES}

This is the process Dino Uses to make the Nitride Windows for us.

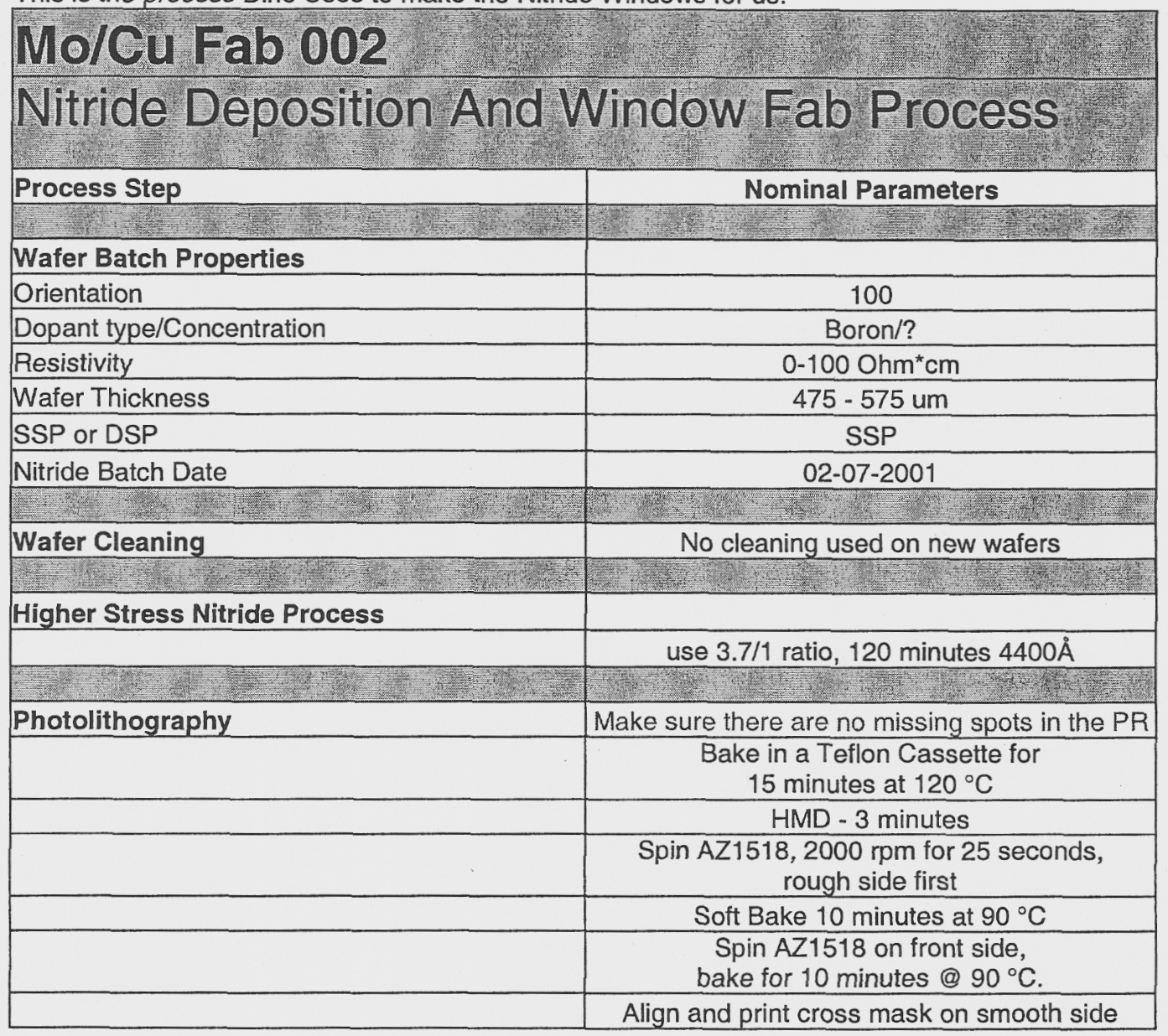


Use $7.5 \times 40=300 \mathrm{mj} / \mathrm{cm} 2$

\begin{tabular}{|c|c|}
\hline & Use $7.5 \times 40=300 \mathrm{mj} / \mathrm{cm} 2$ \\
\hline & $\begin{array}{c}\text { Develop in AZ 1:1 Developer } 45 \text { seconds, } \\
\text { water rinse, blow dry }\end{array}$ \\
\hline & Backside align window mask to cross targets \\
\hline & $300 \mathrm{mj} / \mathrm{cm} 2$ expose \\
\hline & $\begin{array}{l}\text { Develop in } A Z 1: 1 \text { developer } \\
1 \text { min water rinse - blow dry }\end{array}$ \\
\hline 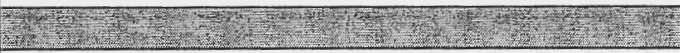 & 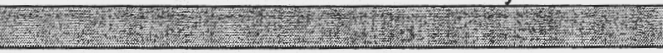 \\
\hline RIE Etch Front Side & $\mathrm{CF} 4+10 \% \mathrm{O} 2$ \\
\hline & 200 mTorr \\
\hline & 150 watts \\
\hline & 30 seconds \\
\hline & This etches $1500 \AA$ into the $4400 \AA$ nitride \\
\hline (2) & 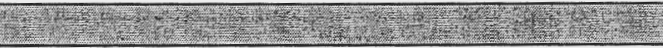 \\
\hline RIE Etch Back Side & $\mathrm{CF} 4+10 \% \mathrm{O} 2$ \\
\hline & 200 mTorr \\
\hline & 150 watts \\
\hline & 180 seconds \\
\hline & This etches $13200 \AA$ into the $4400 \AA$ nitride \\
\hline S. & 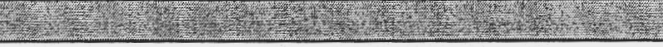 \\
\hline Clean Wafers & \\
\hline Time in Acetone & $?$ \\
\hline & Water Rinse \\
\hline 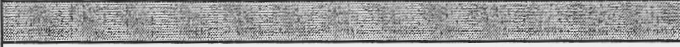 & 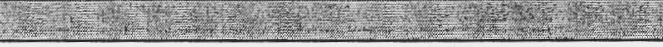 \\
\hline $\mathrm{KOH}$ etch of $\mathrm{Si}$ & $44 \% \mathrm{KOH}$ by wt. \\
\hline & $65^{\circ} \mathrm{C}$ \\
\hline & Etch rate approximately $16 \mu / \mathrm{hr}$ \\
\hline Time in $\mathrm{KOH}$ & $?$ \\
\hline 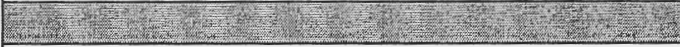 & S \\
\hline Clean Wafers & \\
\hline Time in Piranha Etch & $20 \mathrm{~min}$ \\
\hline & Water Rinse \\
\hline 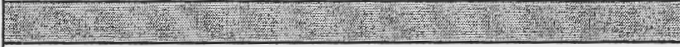 & (3) \\
\hline Measure Stress and Thickness & \\
\hline Nitride Thickness (Å) & 5000 \\
\hline Nitride Stress (Mpa) Compressive or Tensile & 785 Tensile \\
\hline 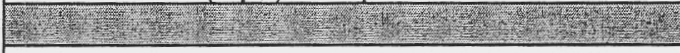 & 20 \\
\hline Notes: & $\begin{array}{l}\text { Thickness of Nitride is accurate to } 5 \% \\
\text { It is measured with a nanospec }\end{array}$ \\
\hline
\end{tabular}


This is the process Troy does to make the multilayers

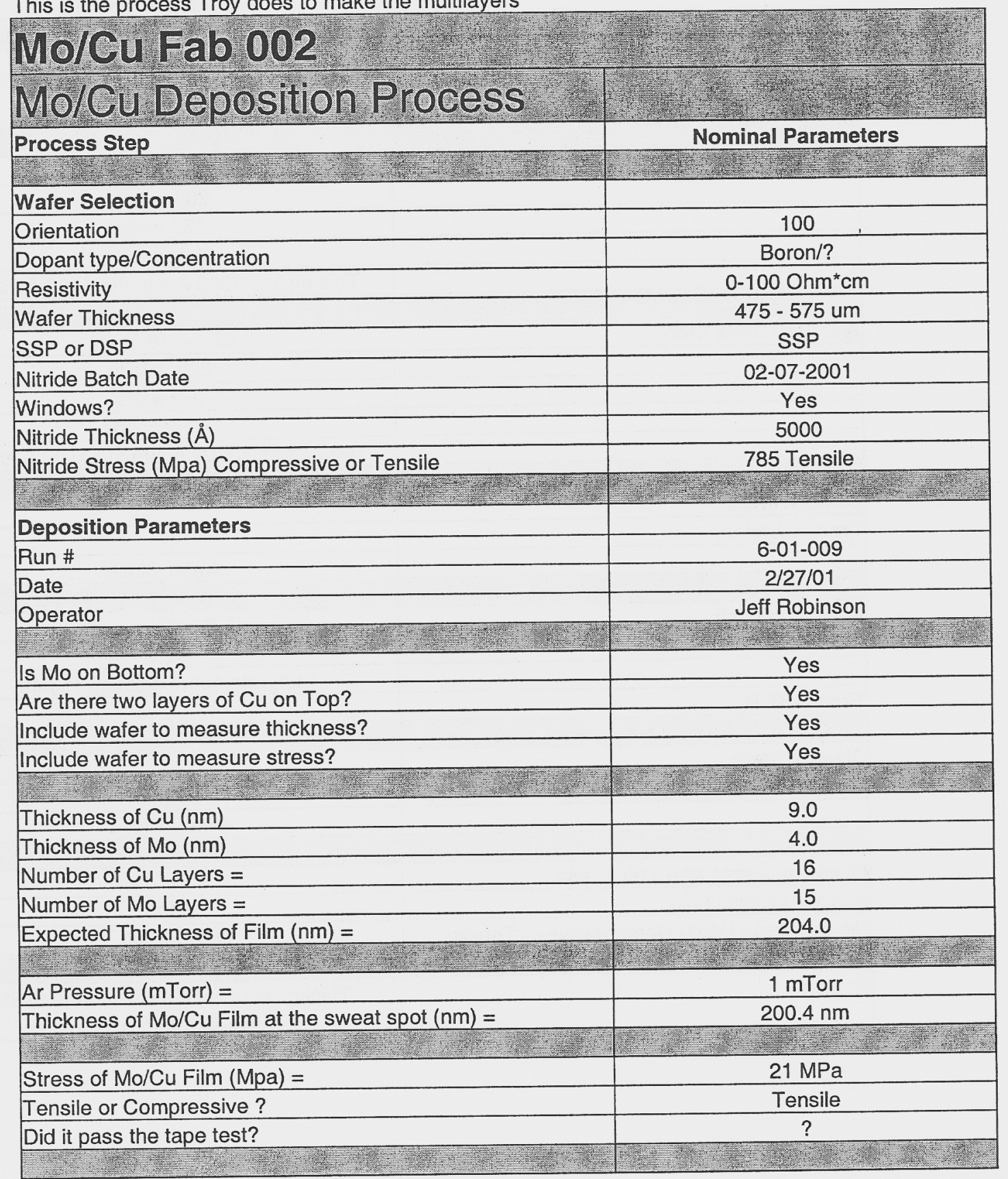


This is the process we do to make the TESs

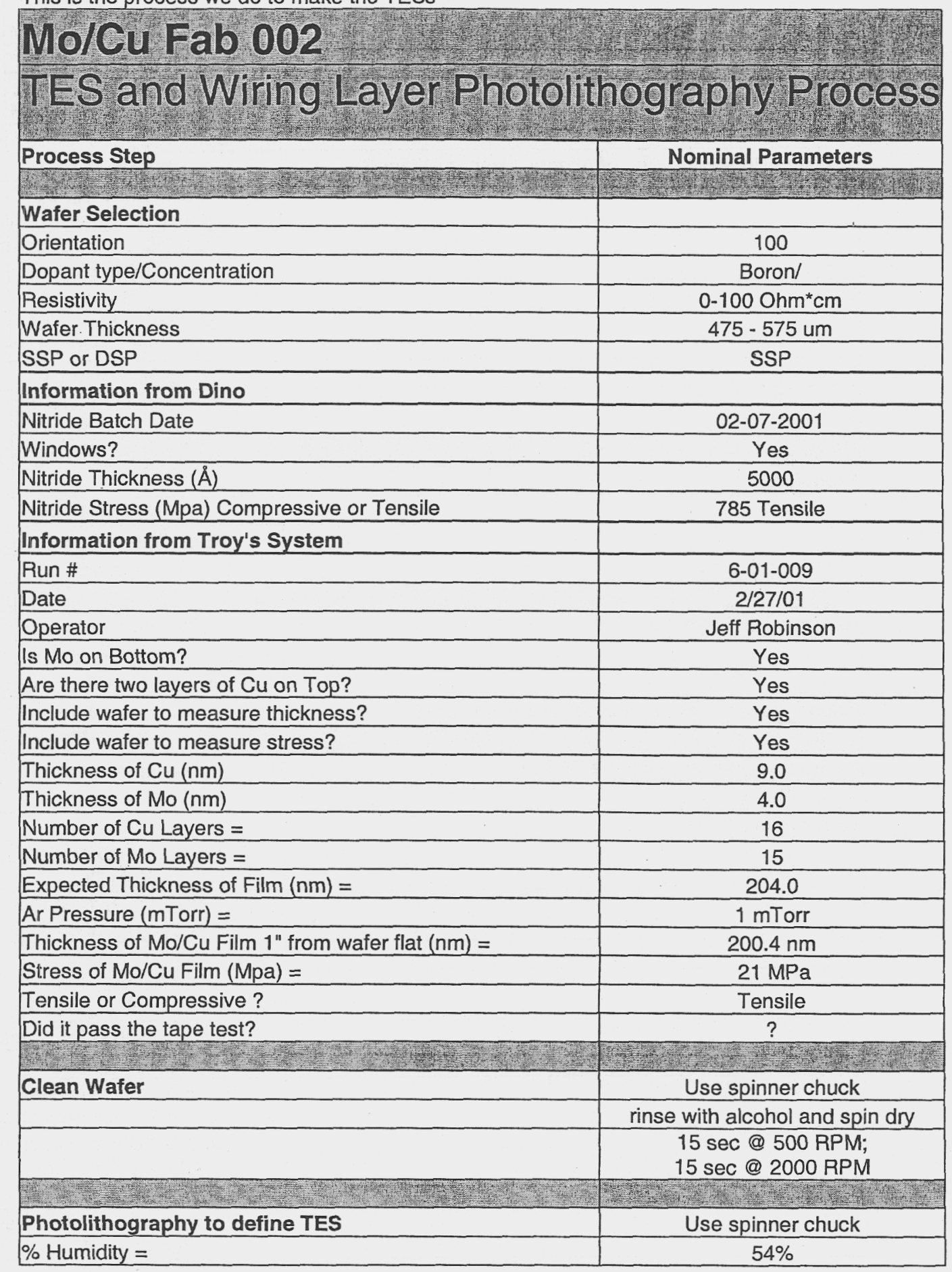




\begin{tabular}{|c|c|}
\hline Temperature $(F)=$ & $66 \mathrm{~F}$ \\
\hline & $\begin{array}{l}\text { Puddle on HMDS for } 30 \mathrm{sec} \text {; } \\
\text { Spin Dry }\end{array}$ \\
\hline & $\begin{array}{c}5 \text { sec @ } 500 \text { RPM; } \\
15 \text { sec @ } 2000 \text { RPM }\end{array}$ \\
\hline & Spin on Photoresist \\
\hline & $\begin{array}{l}15 \mathrm{sec} @ 500 \text { RPM; } \\
30 \mathrm{sec} @ 2000 \text { RPM }\end{array}$ \\
\hline & Soft Bake $90 \mathrm{C}$ for $10 \mathrm{~min}$ \\
\hline Measure Power Reading of Aligner Lamp (mW/cm^2) & $12.42 \mathrm{~mW} / \mathrm{cm}^{\wedge} 2$ \\
\hline & $\begin{array}{c}\text { Exposure Time is } \\
8.0 \mathrm{sec} @ 12.4 \mathrm{~mW} / \mathrm{cm}^{\wedge} 2\end{array}$ \\
\hline & $\begin{array}{c}\text { Turn Off Chuck Vacuum } \\
\text { during Exposure }\end{array}$ \\
\hline Exposure Time $(\mathrm{sec})=$ & $8 \mathrm{sec}$ \\
\hline & $\begin{array}{l}\text { Develop: Dribble for } 5 \mathrm{sec} ; \\
\text { Puddle for } 47 \mathrm{sec}\end{array}$ \\
\hline & Spin Rinse DI \\
\hline & $\begin{array}{l}15 \mathrm{sec} @ 500 \mathrm{RPM} ; \\
15 \mathrm{sec} @ 2000 \mathrm{RPM}\end{array}$ \\
\hline D. & I \\
\hline Etch TES & \\
\hline & Use PAWN Etch \\
\hline & Etch Rate $=20 \mathrm{~nm} / \mathrm{sec}$ \\
\hline Etch Time $(\mathrm{sec})=$ & $22 \mathrm{sec}$ \\
\hline 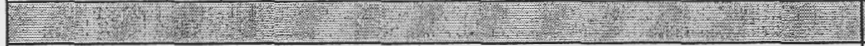 & (2) \\
\hline Remove Photoresist & Use spinner chuck \\
\hline & Puddle Acetone on Wafer for $5 \mathrm{~min}$ \\
\hline & rinse with alcohol and spin dry \\
\hline Inspect Wafer; Take Picture; How does it look? & overetched \\
\hline 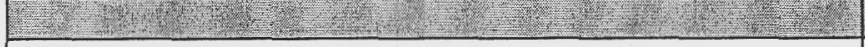 & 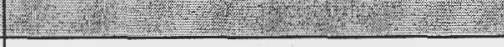 \\
\hline Photolithography to define TES & Use spinner chuck \\
\hline$\%$ Humidity $=$ & $54 \%$ \\
\hline Temperature $(F)=$ & $66 \mathrm{~F}$ \\
\hline & $\begin{array}{c}\text { Puddle on HMDS for } 30 \mathrm{sec} \text {; } \\
\text { Spin Dry }\end{array}$ \\
\hline & $\begin{array}{c}5 \mathrm{sec} @ 500 \mathrm{RPM} ; \\
15 \mathrm{sec} @ 2000 \mathrm{RPM}\end{array}$ \\
\hline & Spin on Photoresist \\
\hline & $\begin{array}{l}15 \mathrm{sec} @ 500 \text { RPM; } \\
30 \mathrm{sec} @ 2000 \text { RPM }\end{array}$ \\
\hline & Soft Bake $90 \mathrm{C}$ for $10 \mathrm{~min}$ \\
\hline Measure Power Reading of Aligner Lamp (mW/cm^2) & $12.42 \mathrm{~mW} / \mathrm{cm}^{\wedge} 2$ \\
\hline & $\begin{array}{c}\text { Exposure Time is } \\
9.0 \mathrm{sec} @ 13.4 \mathrm{~mW} / \mathrm{cm}^{\wedge} 2\end{array}$ \\
\hline & $\begin{array}{l}\text { Turn Off Chuck Vacuum } \\
\text { during Exposure }\end{array}$ \\
\hline Exposure Time $(\mathrm{sec})=$ & $8 \mathrm{sec}$ \\
\hline & $\begin{array}{l}\text { Develop: Dribble for } 5 \mathrm{sec} \text {; } \\
\text { Puddle for } 47 \mathrm{sec}\end{array}$ \\
\hline
\end{tabular}




\begin{tabular}{|c|c|}
\hline & Spin Rinse DI \\
\hline & $\begin{array}{l}15 \text { sec @ } 500 \text { RPM; } \\
15 \text { sec @ } 2000 \text { RPM }\end{array}$ \\
\hline 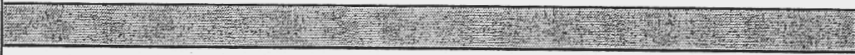 & 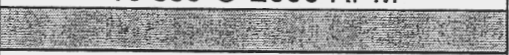 \\
\hline \multicolumn{2}{|l|}{ Deposition of wiring layer } \\
\hline Deposition Run Number & A-01-015 \\
\hline Time Pumping in Load Lock & $25 \mathrm{~min}$ \\
\hline \multicolumn{2}{|l|}{ Ion Gun Surface } \\
\hline Ar Flow Rate SCCM = & $8.8 \mathrm{SCCM}$ \\
\hline $\mathrm{CG7}=$ & 17 \\
\hline CG2 = & NA \\
\hline Baratron $=$ & 0.55 \\
\hline Time lon Gunning the surface = & $\begin{array}{c}30 \mathrm{sec} \text { (10 sec per third of waferl } \\
\text { Rotate Wafer) }\end{array}$ \\
\hline \multicolumn{2}{|l|}{ Deposit Mo Leads } \\
\hline Ar Flow Rate SCCM = & 59.1 \\
\hline$C G 7=$ & 3.2 \\
\hline $\mathrm{CG} 2=$ & 78 \\
\hline Time Sputtering Mo leads = & $10 \mathrm{~min}$ \\
\hline Approximate Leads Thickness $=$ & $200 \mathrm{~nm}$ \\
\hline 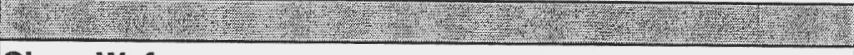 & (2x:- \\
\hline \multirow[t]{3}{*}{ Clean Wafer } & Use spinner chuck \\
\hline & Puddle Acetone on Wafer for $5 \mathrm{~min}$ \\
\hline & rinse with alcohol and spin dry \\
\hline Inspect Wafer; Take Picture; How does it look? & $\begin{array}{l}\text { Wafer looks UGLY; } \\
\text { Photoresist is burned }\end{array}$ \\
\hline 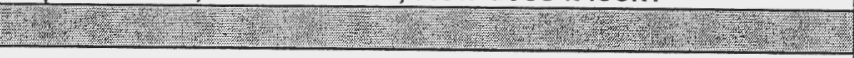 & (1) \\
\hline \multirow{2}{*}{ (1) } & $\begin{array}{l}\text { Have Wafer diced into } \\
1 / 4^{\prime \prime} \times 1 / 4^{\prime \prime} \text { squares }\end{array}$ \\
\hline & $\begin{array}{l}\text { Make sure there is } \\
\text { photoresist on the front }\end{array}$ \\
\hline 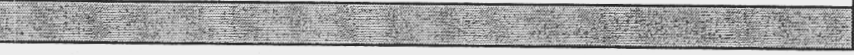 & 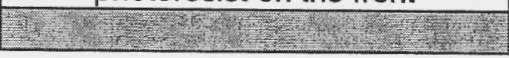 \\
\hline
\end{tabular}

